\title{
WestVirginiaUniversity
}

THE RESEARCH REPOSITORY @ WVU

Graduate Theses, Dissertations, and Problem Reports

2013

\section{Characterization of Immune Responses to Repeated Aspergillus fumigatus Exposures}

Amanda Dawn Buskirk

West Virginia University

Follow this and additional works at: https://researchrepository.wvu.edu/etd

\section{Recommended Citation}

Buskirk, Amanda Dawn, "Characterization of Immune Responses to Repeated Aspergillus fumigatus Exposures" (2013). Graduate Theses, Dissertations, and Problem Reports. 4954.

https://researchrepository.wvu.edu/etd/4954

This Dissertation is protected by copyright and/or related rights. It has been brought to you by the The Research Repository @ WVU with permission from the rights-holder(s). You are free to use this Dissertation in any way that is permitted by the copyright and related rights legislation that applies to your use. For other uses you must obtain permission from the rights-holder(s) directly, unless additional rights are indicated by a Creative Commons license in the record and/ or on the work itself. This Dissertation has been accepted for inclusion in WVU Graduate Theses, Dissertations, and Problem Reports collection by an authorized administrator of The Research Repository @ WVU.

For more information, please contact researchrepository@mail.wvu.edu. 


\title{
Characterization of Immune Responses to Repeated Aspergillus fumigatus Exposures
}

\author{
Amanda Dawn Buskirk
}

Dissertation submitted to the School of Medicine

at West Virginia University

in partial fulfillment of the requirements

for the degree of

Doctor of Philosophy

in

Immunology and Microbial Pathogenesis

Donald H. Beezhold, Ph.D., Chair

Kathleen M. Brundage, Ph.D.

Slawomir Lukomski, Ph.D.

Rosana Schafer, Ph.D.

Paul D. Siegel, Ph.D.

Department of Microbiology, Immunology and Cell Biology

Morgantown, West Virginia

2013

Keywords: Aspergillus fumigatus, fungi, pigment, melanin, animal models, Tc17 cells, aerosol exposures 


\begin{abstract}
Characterization of Immune Responses to Repeated Aspergillus fumigatus Exposures

Amanda Dawn Buskirk
\end{abstract}

Personal exposures to A. fumigatus are associated with a variety of adverse health outcomes, including invasive aspergillosis, allergic sensitization, and asthma. Due to the high rate of mortality associated with invasive disease in immunocompromised patients, most studies of A. fumigatus have aimed to characterize the immune responses in immunocompromised murine models. However, a larger portion of the population is affected by fungal-induced allergies and asthma and the immune mechanisms associated with exposure have rarely been studied in an immunocompetent model. These models do not accurately reflect the natural method of exposure to environmental sources of conidia, and may significantly impact responses between fungi and the host immune system. Furthermore, little is known about the mechanisms associated with fungal induced allergy and asthma. Persistence of antigen is believed to play a role in induction of these diseases. Melanin, an A. fumigatus virulence factor, protects conidia from innate clearance, leading to fungal persistence. However, it remains unknown if melanin influences the induction of A. fumigatus - specific allergy and/or asthma. In these studies, immunocompetent mice were repeatedly exposed to A. fumigatus wild-type (WT) or melanin deficient conidia via aspiration or dry conidial exposures via an acoustical generator. Histopathological analysis of lung sections showed moderate to severe inflammation in all exposed mice, regardless of the exposure method or melanin content of the conidia. Overall, flow cytometric analysis of bronchoalveolar lavage fluid was similar between the exposure methods. However, there were marked differences between WT and melanin-deficient exposure groups in many of the cell populations analyzed. Germination was evident in all mice exposed to WT conidia, despite the exposure method. However, melanin-deficient conidia did not germinate, and were also cleared more rapidly in both exposure groups. Enhanced clearance of the melanin-deficient conidia was evident in mice exposed via the acoustical generator when compared to the aspiration exposure groups. Importantly, CD8 ${ }^{+} \mathrm{IL}-17^{+} \mathrm{Tc} 17$ cells were elevated above control groups in all exposed mice, regardless of the exposure method, though the numbers were significantly increased in mice exposed to WT conidia. This is a significant finding, as the presence of these cells has not been previously reported in the context of $A$. fumigatus-induced immune responses. Evidence also suggests that the induction of Tc17 cells may be influenced by WT germination. Taken together, the data presented in this dissertation are among the first to characterize the immune responses to repeated dry fungal exposures in immunocompetent animals. Because these studies proved the feasibility of the dry aerosol model for repeated exposures, future studies with different environmentally prevalent fungi can be adapted for use with the acoustical generator to provide more accurate analysis of immune responses following repeated dry exposures. 
To my loving parents, Roger and Bonnie, who taught me that there was nothing I could not accomplish without hard work and dedication, and whose support and guidance carried me through it all. 


\section{ACKNOWLEDGEMENTS}

Throughout my graduate school experience, I have had the pleasure of working with numerous talented people. I would like to take this opportunity to acknowledge and extend my heartfelt gratitude to all of those who have helped make this dissertation possible. First of all, I'd like to thank my advisor, Don Beezhold, for his patience, understanding, and superior guidance throughout my years in his laboratory. He has been an exceptional mentor who has taught me many valuable lessons both scientifically and personally. I would also like to thank my committee members for their extra time, guidance, and support. Many thanks go out to the members of the ACIB lab at NIOSH. In particular, I owe much gratitude to Steve Templeton and Brett Green, who never failed to keep me on track with kind words of encouragement and support, and who have taught me valuable lessons that I will carry with me always. Ajay Nayak and Angie Lemons at NIOSH also deserve many thanks for their help with experiments, scientific discussions, and most importantly, their friendship. Additionally, I would like to thank all the friends I have made throughout my journey in Morgantown.

On a more personal level, I have been blessed with the best family and friends. I would like to thank my church family who prayed continuously for me, and God who answered those prayers. I would also like to extend my deepest gratitude and appreciation to my parents, Bonnie and Roger. Thank you, from the bottom of my heart, for all of your continuous support, encouragement, and sacrifices that have brought me where I am today. In addition to my parents, my family and extended family deserve more acknowledgement and thanks than the words on this paper can illustrate. Charlotte, Jack, and Roger, thank you for always listening when I needed an ear, and offering a home away from home. Thanks to you and my wonderful nieces and nephews for never letting me forget why I chose this path. To Liz, you have been an amazing source of comfort, support, and encouragement throughout all of the successes and failures, and words could never describe how thankful I am for you. To Kelly, I want to thank you for being you, for keeping me grounded, and staying by my side since this new reality was just a far off dream. To Shana, thanks for being a wonderful friend, always keeping me humble and laughing, and spending countless hours helping me study. And though this may seem cliché, thanks to my wonderful Reesey dog, who never left my side through all of the studying and writing, who listened to all of my rants, and whose loyalty is far beyond what I've ever deserved. You are all amazing and I couldn't have done this without each one of you! 


\section{Table of Contents}

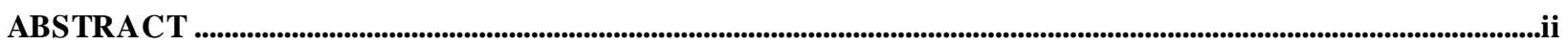

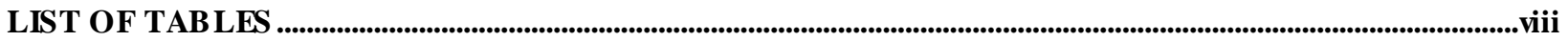

LIST OF FIGURES ........................................................................................................................................................................ix

LIST OF ABB REVIATIONS .............................................................................................................................................................................

CHAPTER 1 LITERATURE REVIEW............................................................................. 1

I. Aspergillus fumigatus Over view................................................................................................................................................2

II. Respiratory and Pathogenic A. fumigatus Diseases..................................................................................................................5

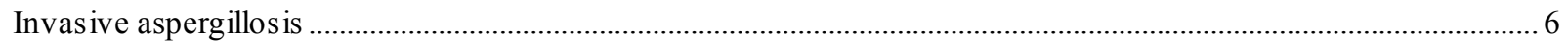

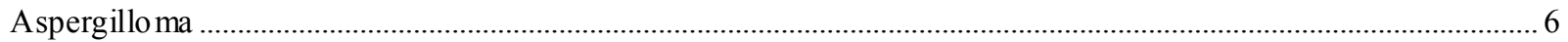

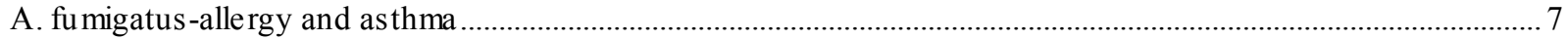

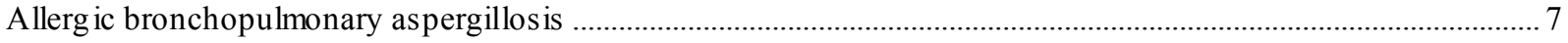

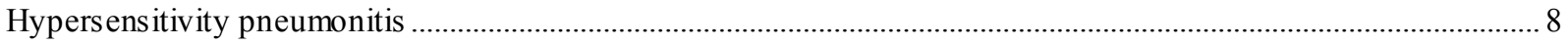

III. Host-Fungal Interactions/ In nate Immunity ................................................................................................................................8

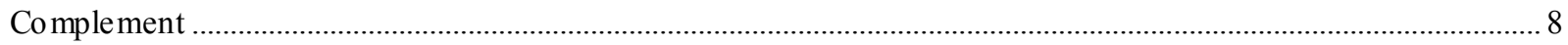

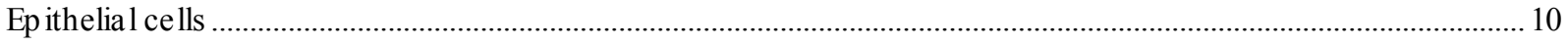

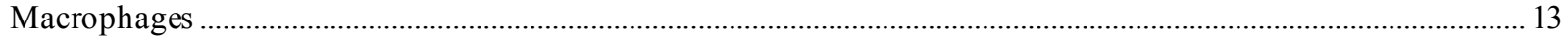

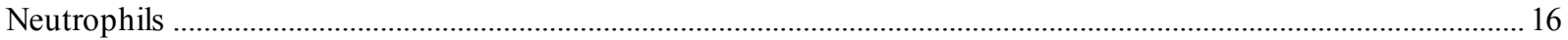

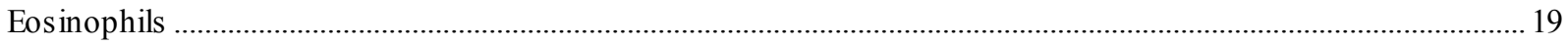

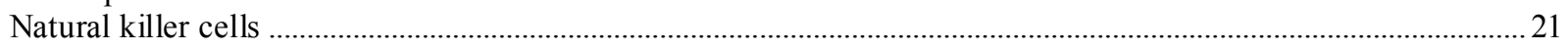

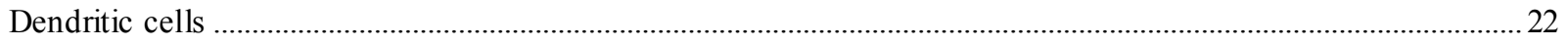

IV. A. fumigatus-Induced Adapti ve Immunity ..........................................................................................................................25

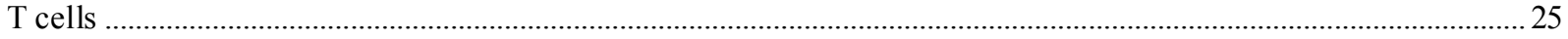

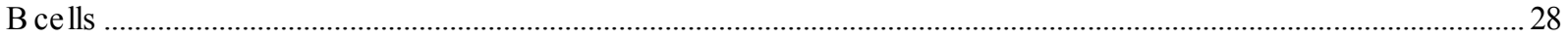

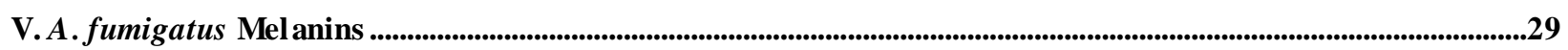

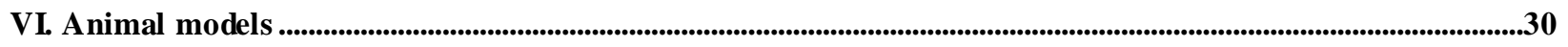

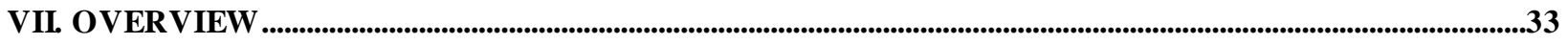

REFERENCES ........................................................................................................................................................................................35

CHAPTER 2 PULMONARY IMMUNE RESPONSES TO ASPERGILLUS FUMIGATUS CONIDIA IN AN IMMUNOCOMPETENT MOUSE MODEL OF REPEATED ASPIRATION EXPOSURES

INTRODUCTION ............................................................................................................................................................................46

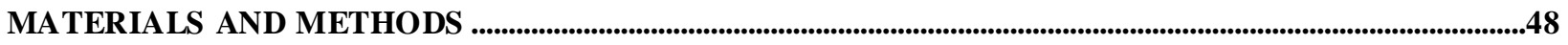

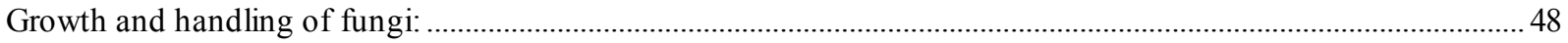

MALDI-qTOF MS analysis of me lanin mutant conidia: ............................................................................................ 48 
Polyacrylamide Gel Electrophores is:

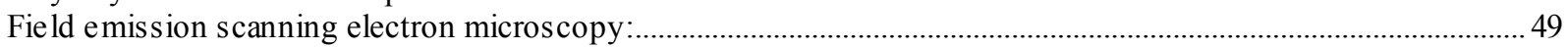

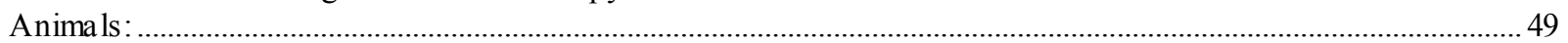

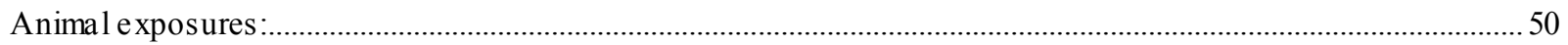

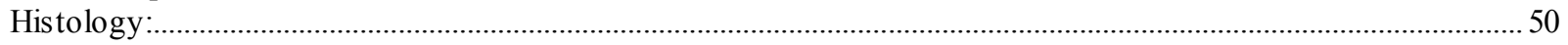

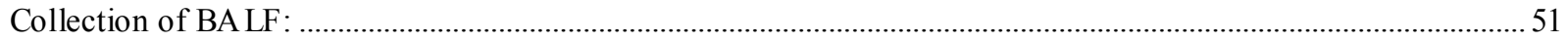

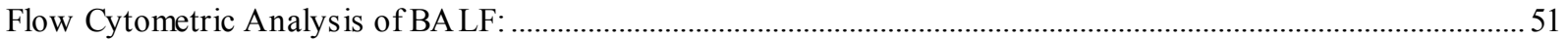

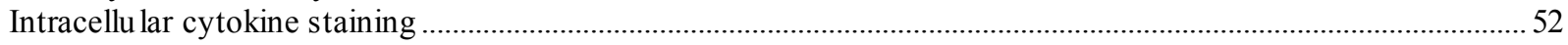

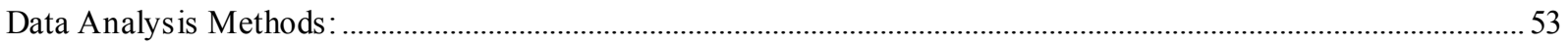

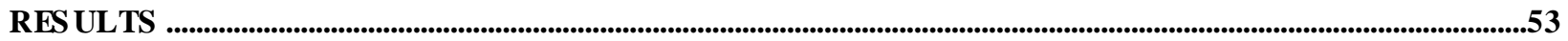

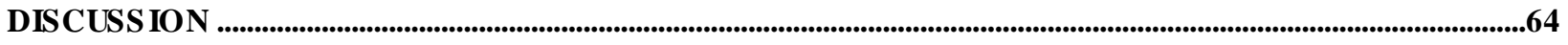

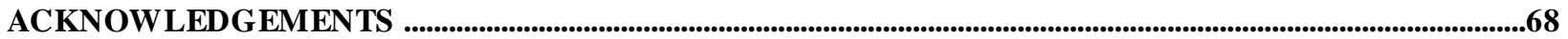

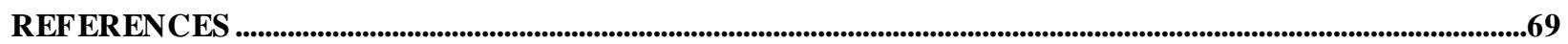

\section{CHAPTER 3 DEVELOPMENT AND CHARACTERIZATION OF A MURINE MODEL OF} REPEATED DRY FUNGAL EXPOSURE ........................................................................

INTRODUCTION ............................................................................................................................................................................74

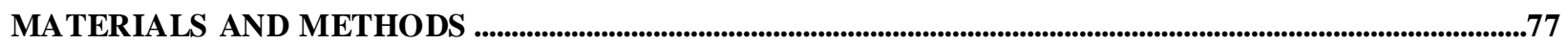

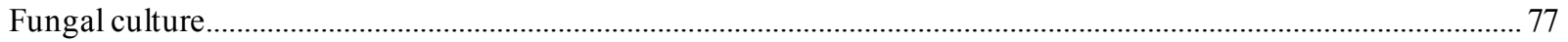

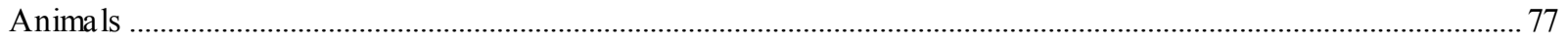

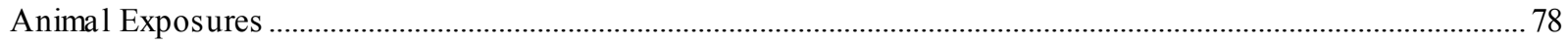

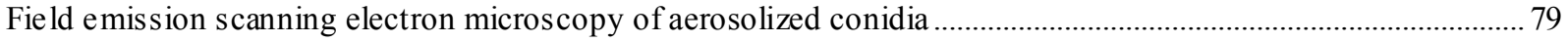

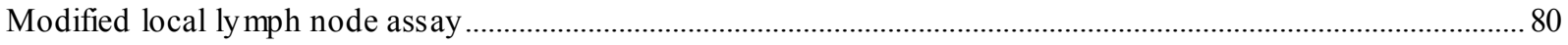

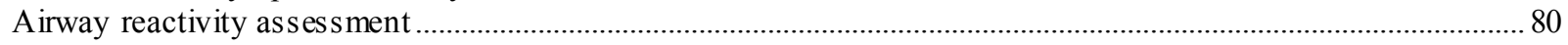

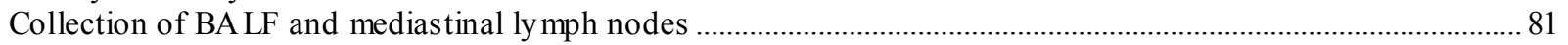

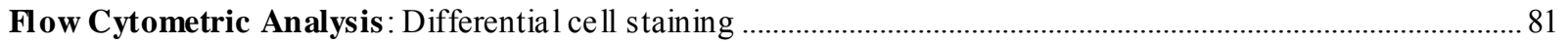

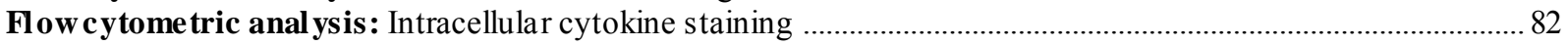

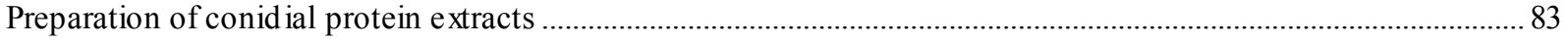

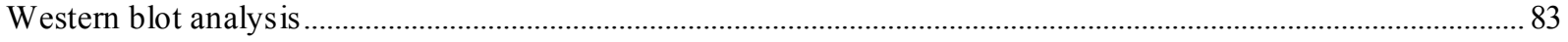

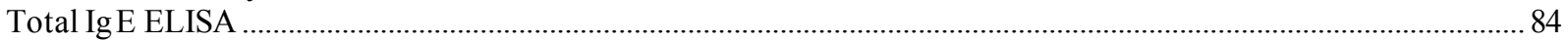

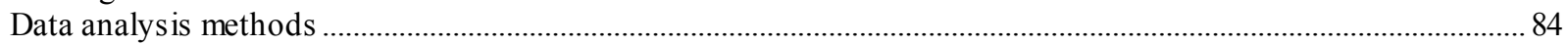

RES ULTS

DISCUSS ION

REFERENCES

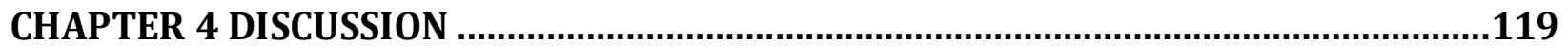

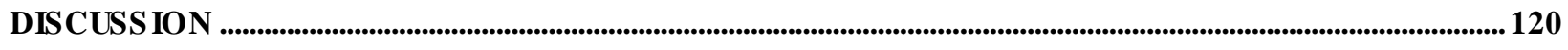

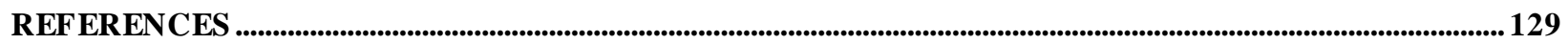

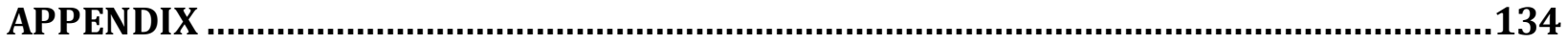




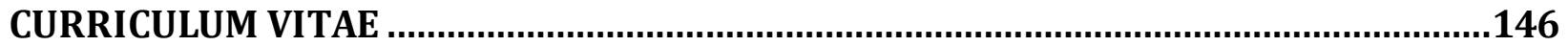




\section{LIST OF TABLES}

Table

Page

Table 1.1 - A. fumigatus antigens $(24,26)$ 


\section{LIST OF FIGURES}

Figure 2.1. + MALDI qTOF MS fingerprint mass spectra ................................................... 55

Figure 2.2 Field emission scanning electron microscopy images......................................... 56

Figure 2.3 Exposure schedule and characterization of lung inflammation ................................ 58

Figure 2.4 T cell cytokine production following multiple aspirations. .......................................6 60

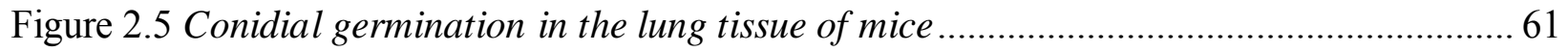

Figure 2.6 Rate of conidial clearance in exposed mice. ...........................................................6. 63

Figure 3.1 Preliminary dry particle generator exposure chamber .......................................... 86

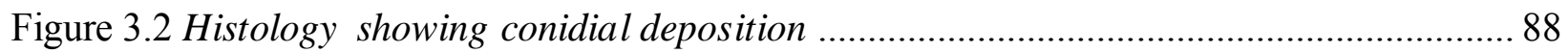

Figure 3.3 Local Lymph Node Assay ................................................................................. 90

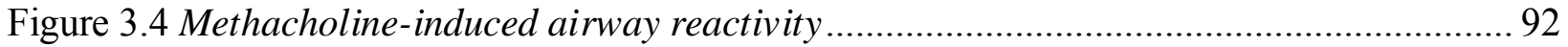

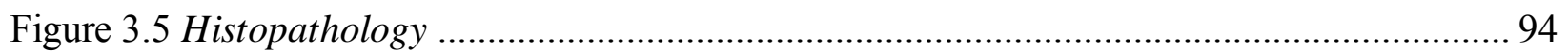

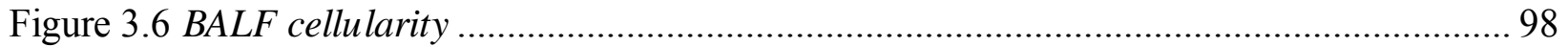

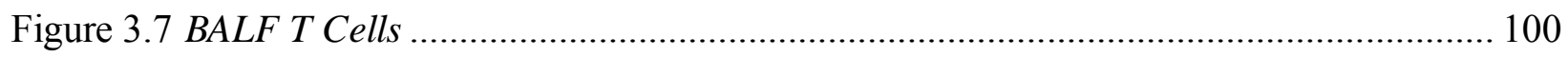

Figure 3.8 Total cell counts in the mediastinal lymph nodes ............................................... 102

Figure 3.9 Intracellular cytokine flow cytometric analysis of the mediastinal lymph nodes...... 104

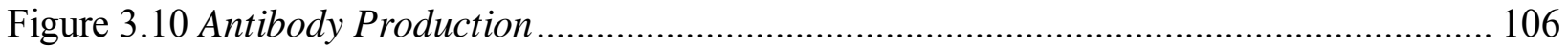




\section{LIST OF ABBREVIATIONS}

$\Delta a l b 1$

$\triangle a r p 2$

AAM

ABPA

AR

APC

BALF

BMDCs

CCL

CCR

CD

$\mathrm{CF}$

CGD

CXCL

DC-SIGN

DNA

DCs

ELISA

FHL-1

GM-CSF

GPI

HIV

HLA albino A. fumigatus mutant

$\tan$ A. fumigatus mutant

alternatively activated macrophages

allergic bronchopulmonary aspergillosis

airway reactivity

antigen presenting cells

bronchoalveolar lavage fluid

bone marrow-derived dendritic cells

chemokine ligand

chemokine receptor type

cluster of differentiation

cystic fibrosis

chronic granulomatous disease

chemokine ( $\mathrm{C}-\mathrm{X}-\mathrm{C}$ motif) ligand

dendritic cell-specific ICAM-grabbing non-integrin

deoxyribonucleic acid

dendritic cells

enzyme-linked immunosorbent assay

Factor H-like protein 1

granulocyte, macrophage-colony stimulating factor

glycophosphatidylinositol

human immunodeficiency virus

human leukocyte antigen 


\begin{tabular}{|c|c|}
\hline HP & hypersensitivity pneumonitis \\
\hline IFN & interferon \\
\hline IL & interleukin \\
\hline $\mathrm{LN}$ & lymph node \\
\hline LPS & lipopolysaccharide \\
\hline ICAM & intracellular adhesion molecule \\
\hline MAC & membrane attack complex \\
\hline MALDI qTOF MS & $\begin{array}{l}\text { matrix-assisted laser desorption/ionization quadrupole time-of- } \\
\text { flight mass spectrometry }\end{array}$ \\
\hline MHC & major histocompatibility complex \\
\hline MMP & matrix metalloproteinase \\
\hline MyD88 & myeloid differentiation primary response gene 88 \\
\hline NETs & neutrophil extracellular traps \\
\hline NK cells & natural killer cells \\
\hline PAMPs & pathogen-associated molecular patterns \\
\hline $\mathrm{pDCs}$ & plasmacytoid dendritic cells \\
\hline PMNs & polymorphonuclear cells \\
\hline PRRs & pattern recognition receptors \\
\hline RNA & ribonucleic acid \\
\hline Rod & rodlet layer \\
\hline ROS & reactive oxygen species \\
\hline Tc17 & $\mathrm{CD} 8^{+} \mathrm{IL}-17^{+}$producing $\mathrm{T}$ cells \\
\hline TLRs & toll-like receptors \\
\hline TNF & tumor necrosis factor \\
\hline VCAM-1 & vascular cell adhesion molecule- 1 \\
\hline
\end{tabular}


wild-type 


\section{Chapter 1}

Literature Review 


\section{Literature Review}

Due to the clinical impact of $A$. fumigatus-induced diseases, multiple animal models have been developed to characterize host-fungal interactions, identify cell types and mechanisms important in clearing the fungus, and develop better diagnostics for earlier detection leading to better prognoses. To date, most A. fumigatus animal models have focused primarily on the mechanisms of invasive disease in immunocompromised animals and use fungal suspensions or extracts for exposures; however, the impact of dry, A. fumigatus exposures, as occurs naturally, in competent subjects and the development of allergy and asthma remains uncharacterized. Despite the quantity of available information concerning host-fungal interactions; two important caveats remain: 1) Unnatural exposure models, and 2) a poor understanding of the phenotypic characteristics that may influence the development of adverse health effects including allergy and/or asthma. The primary hypothesis of this dissertation was that exposures to dry A. fumigatus conidia that more closely represent natural exposures would result in differences in the murine immune responses when compared to the conventional fungal suspension aspiration exposures. Further, melanin, a known A. fumigatus virulence factor, protects conidia from innate clearance leading to persistence within the host. Thus, a secondary hypothesis was that murine exposures to melanized conidia would modulate immune responses, leading to a greater potential for the induction of allergy and asthma. In this dissertation, the goals were to: 1) develop an immunocompetent murine model of repeated aerosol exposures to dry fungal conidia that more closely resembles natural environmental exposures, 2) examine the impact of fungal melanization on the induction of an allergic phenotype, and 3) identify the fungal components that induce a specific antibody response against A. fumigatus conidia.

\section{Aspergillus fumigatus Overview}

A. fumigatus exposures can lead to a variety of adverse health effects, making it an important pathogen with an enormous impact on human health (1). A. fumigatus is an environmentally ubiquitous fungus, commonly associated with decaying plant matter $(2,3)$. The fungus propagates through forming unicellular conidia (amerospores) that are 2-3 $\mu \mathrm{m}$ in diameter and emerge through the formation of a conidiophore from vegetative hyphae (2). The small size and aerodynamic nature of these amerospores allow them to be easily aerosolized and 
disseminate over long distances following common environmental disturbances, such as wind and vibration (2).

The conidial cell wall is traditionally thought to be composed of four layers including a phospholipid bilayer, chitin, $\beta$-glucan, and mannose proteins. However, recent studies have shown that the outermost layer of A. fumigatus is composed of a rodlet layer that consists of hydrophobic proteins, rodlet $A$ and $\operatorname{rodlet} B(\operatorname{rod} A$ and $\operatorname{rodB})(4)$. This layer has been shown to be immunologically inert (4-6). The $\operatorname{rodA}$ and $\operatorname{rodB}$ proteins bundle together to form fibers that are woven together on the surface of conidia and envelope an underlying melanin layer $(4,5)$. The melanin layer is composed of large biopolymers, extensively intercalated throughout the conidial wall. Melanins have been identified as a virulence factor in several fungal pathogens including A. fumigatus and are responsible for the characteristic blue-green color observed in $A$. fumigatus conidia (7-12). Under the melanin layer is the conidial wall that is composed of carbohydrates, $\alpha$ - and $\beta$-glucans, chitin, galactomannans, and other polysaccharides (13). These compounds have been identified as pathogen-associated molecular patterns (PAMPs). Upon recognition by pattern recognition receptors (PRRs), PAMPs induce a cascade of events which shape and/or modulate the immune response(s) against the fungus (14). Specifically, recognition of $\beta$-glucans is known to suppress toll-like receptor- 4 (TLR-4) induced responses, while $\alpha$ glucans inhibit the production of IL-6 through TLR-2 and TLR-4 stimulation (15). Galactomannans were shown to diminish the activity of TLR-4 stimulation, but had limited effects on TLR-2. Chitin, however, does not appear to have immunomodulatory activity (15).

The vegetative growth phase of the A. fumigatus may be induced following deposition on environmental substrates that may include temperatures between $20-70^{\circ} \mathrm{C}$, high water content, and substrates rich in sugars, proteins, and/or carbohydrates $(3,16,17)$. Importantly, germination has been identified as an important component which directly affects the ensuing immuneresponse against the fungus $(18,19)$. Conidial germination begins as the conidia swell, lose their rodlet and melanin layers, and subsequently form germ tubes (3). During this stage of active hyphal growth, PAMPs become more accessible and numerous proteases, including serine proteases, alkaline proteases, and elastases, are produced by the fungus $(20,21)$. These proteases are secreted to aid in fungal anchoring and to degrade the substrate to release nutrients that can be utilized by the fungus. Further, during hyphal growth, mycotoxins, such as proteases and gliotoxin, are secreted which can have adverse effects on immune cells (21-23). A. fumigatus 
produces many proteins that can be allergens $(22,24,25)$. In general, these allergens are predominantly serine, aspartate, and alkaline proteases, matrix metalloproteinases, heat shock proteins, enolase, ribosomal proteins, and cell wall proteins (Table 1.1). However, the function of many of the 22 identified allergens remains unknown.

\section{Table 1.1}

\begin{tabular}{|c|c|c|}
\hline \multicolumn{3}{|c|}{ A. fumigatus antigens $(24,26)$} \\
\hline Identity & Function & Response \\
\hline $1,3-\beta$ glucanosyltrans ferase & cell wall biosynthesis & $\begin{array}{l}\text { Th1/Treg } \\
\text { \&Th2 }\end{array}$ \\
\hline$\beta$ 1,6 glucan-chitin linkages & cell wall biosynthesis & Th1/Treg \\
\hline aspartic protease (Peplp) & secreted protease & Th1/Treg \\
\hline metalloprotease (Mep1p) & secreted protease & Th2 \\
\hline mycelial catalase I (Cat1p) & secreted protease & Th2 \\
\hline superoxide dismutase (Sod1p) & secreted protease & Th2 \\
\hline dipeptidylpeptidase V (Dpp5p) & secreted protease & Th2 \\
\hline ribonuclease (RNUp) & secreted protease & Th2 \\
\hline $\begin{array}{l}\text { glycosylinositolphosphoceramide } \\
\text { (GSL) B }\end{array}$ & glycolipid & Th2/Th17 \\
\hline $\begin{array}{l}\text { GPI-anchored } \\
\text { lipophospho galactomannan }\end{array}$ & glycolipid & Th2/Th17 \\
\hline$\alpha 1-3$ glucan & polysaccharide & Th1 \\
\hline$\beta 1-3$ glucan & polysaccharide & Th1/Th17 \\
\hline galactomannan & polysaccharide & Th2/Th17 \\
\hline Asp fl & major allergen $1 /$ ribotoxin & Th2 \\
\hline Asp f2 & allergen 2 & Th2 \\
\hline
\end{tabular}




\begin{tabular}{|c|c|c|}
\hline Asp $\mathrm{f3}$ & peroxisomal membrane protein & Th1 \\
\hline Asp f4 & allergen 4 & Th2 \\
\hline Asp f5 & metalloprotease & Th2 \\
\hline Asp f6 & manganese superoxide dismutase & Th1 \\
\hline Asp f7 & allergen 7 & Th2 \\
\hline Asp f8 & acidic ribosomal protein $\mathrm{P} 2$ & unknown \\
\hline Asp $9 / 16$ (Crfl) & cell wall glucanase & Th1 \\
\hline Asp fl0 & aspartic endopeptidase & Th1/Treg \\
\hline Asp fl 1 & $\begin{array}{l}\text { cyclophillin type peptidyl-prolyl } \\
\text { isomerase }\end{array}$ & Th1/Th2 \\
\hline Asp fl2 & heat shock protein hsp90 family & Th1/Th2 \\
\hline Asp fl3 & allergen $13 /$ cerato platanin (protease) & $\mathrm{Th} 2$ \\
\hline Asp fl 7 & $\begin{array}{l}\text { galactomannoprotein MP1/ק- } \\
\text { glucanase }\end{array}$ & Th1/Th2 \\
\hline Asp fl 8 & alkaline serine protease Alp2 & Th2 \\
\hline Asp $f 22$ & enolase & Th1 \\
\hline Aspf23 & 605 ribosomal protein L3 & unknown \\
\hline
\end{tabular}

\section{Respiratory and Pathogenic A. fumigatus Diseases}

Due to the ubiquitous nature of $A$. fumigatus, are routinely exposed up to $10^{4} \mathrm{~A}$. fumigatus conidia $/ \mathrm{m}^{3}$ (2). This number can be increased in water-damaged buildings and occupational settings to greater than $10^{10}$ conidia $/ \mathrm{m}^{3}$ (2). Repeated exposures to A. fumigatus have been associated with multiple diseases, including allergy, asthma, hypersensitivity pneumonitis (HP), allergic rhinitis, allergic bronchopulmonary aspergillosis (ABPA), and invasive aspergillosis (3, 27-30). 


\section{Invasive aspergillosis}

A. fumigatus-associated invasive diseases have been steadily increasing in association with the development of improved therapeutic interventions for immunosuppressed, critically-ill patients. Several factors contribute to the occurrence of invasive disease including prolonged neutropenia, transplantations (bone marrow and solid organ), human immunodeficiency virus (HIV), hematologic malignancies, chemotherapy, corticosteroid treatment, and other immune deficiencies. Overall, there has been a steady increase in the frequency of invasive fungal diseases from 1-7\% between 1978-1992, while the diagnosis of invasive aspergillosis has increased from 17-60\% of all mycoses identified at autopsy $(31,32)$. The incidence of invasive aspergillosis in the United States is 36 per million hospital patients, with neutropenic patients being particularly susceptible (32). Two different forms of invasive A. fumigatus disease have been characterized;1) chronic necrotizing aspergillosis and 2) invasive aspergillosis (3, 33). Invasive aspergillosis is the predominant form of invasive disease studied epidemiologically and in animal models.

\section{Aspergilloma}

Personal exposures to A. fumigatus can also result in the opportunistic colonization of the dermis, mucosa, and airways. The conditions associated with this include dermatitis, keratitis, and the formation of aspergillomas (34). While dermatitis and keratitis are considered the less severe forms of colonization, aspergilloma is a more serious form of persistent A. fumigatus colonization. Symptoms of aspergilloma include labored breathing, cough, fever, weight loss, and haemoptysis, the latter of which is associated with mortality rates up to $26 \%(35,36)$. A cavitary aspergilloma is defined by persistent colonization and the formation of a hyphal mass within the lungs or sinuses. This typically occurs in immunocompetent individuals. Cavities most commonly form in patients that have previously been diagnosed with tuberculosis, though cavitary aspergillomas have also been shown to form in those diagnosed with other pulmonary and lung-related diseases $(35,37)$. Importantly, the mechanisms associated with aspergilloma are poorly understood, though insufficient drainage of respiratory cavities is thought to be a major factor. Furthermore, treatment is problematic, as common antifungal agents often fail to clear the organism, and surgical excision of the hyphal mass is required (35). Therefore, aspergilloma 
formation is unable to be prevented in patients with irregular lung structure, and once fungal persistence occurs, it is difficult to clear with current antifungal agents $(35,37)$.

\section{A. fumigatus-allergy and asthma}

While invasive fungal infections have been the primary focus of most research, there has been an increase in research on $A$. fumigatus allergy $(25,38)$ Fungal allergic sensitization has been reported to be between $6-24 \%$ in the general population, with reports of A. fumigatusspecific allergy in $23-80 \%$ of asthmatic patients $(25,39-41)$. The prevalence increases to $44 \%$ in atopics, and up to $80 \%$ in asthmatic patients in the US $(42,43)$. Age is also a factor in the frequency of fungal allergies, as $45 \%$ of pediatric asthmatics suffer from fungal allergies, while this increases to $70 \%$ of adult asthmatics (44). A. fumigatus-specific allergies have been reported in up to $2.4 \%$ of the general population, and $5-27.6 \%$ of atopics, thereby highlighting its importance as a prevalent fungal allergen $(42,45)$. Allergic rhinitis, sometimes referred to as hay fever, is commonly exacerbated by A. fumigatus. Symptoms include sneezing, itching, rhinorrhea, and nasal congestion. Allergic rhinitis is mediated by type I hypersensitivity, is associated with a genetic predisposition (atopy), and often precedes the development of asthma.

\section{Allergic bronchopulmonary aspergillosis}

Allergic bronchopulmonary aspergillosis (ABPA) a complex allergic responses resulting from exposures to A. fumigatus (40). The disease is characterized by hyperactive proinflammatory immune responses, and type I and III hypersensitivity reactions. ABPA does not result in fungal colonization. It occurs predominantly in chronic asthmatics and cystic fibrosis (CF) patients, with estimates of prevalence between 2-14\% of corticosteroid-treated asthmatics and $7-15 \%$ of $\mathrm{CF}$ patients $(40,46)$. Other reports have indicated higher frequencies up to $40 \%$ in patients with chronic asthma, and up to $38 \%$ in acute asthmatics (40). Life-threatening ABPA has been reported in $12-40 \%$ of asthmatic patients $(25,40)$. Clinical symptoms used for ABPA diagnosis include asthma, immediate skin reactivity to A. fumigatus antigens, dilated bronchi in the inner two-thirds of the chest cavity, peripheral eosinophilia, and necrotizing pneumonia (3). Currently, the immunological mechanisms leading to the induction and immunopathogenesis of ABPA are not well understood; however, an imbalanced $\mathrm{T}_{\mathrm{H}} 2$ response, as well as genetic predisposition, has been hypothesized to drive disease induction and 
progression $(40,46)$. To date, patient treatment has been hindered due to lack of consistent clinical diagnostic standards; therefore, an improved understanding of the mechanisms of ABPA induction are required to develop better diagnostics for clinical usage.

\section{Hypersensitivity pneumonitis}

A. fumigatus is also an etiological agent of hypersensitivity pneumonitis (HP), a rare, allergic, interstitial lung disease commonly associated with occupational exposures (47). HP occurs due to exposure to foreign antigens and results in chronic pulmonary inflammation. It has been reported in $2-13 \%$ of interstitial lung disease reports, with an annual occurrence of 30 per 100,000 patients $(47,48)$. To date, the extent of exposure and the immunological mechanism(s) behind the induction of HP remain poorly understood.

\section{Host-Fungal Interactions/ Innate Immunity}

Humans inhale over 10,000 liters of air each day, containing numerous bacteria, viruses, fungi, toxins, allergens, and other pollutants (49). Since A. fumigatus conidia are ubiquitous, and routinely inhaled, it is necessary to review common immune mechanisms used for conidial clearance to begin to understand how exposures may result in the aforementioned disease states. Because inhalation is the predominant route of exposure, and the focus for the research presented in this dissertation, only the impact of A. fumigatus exposures on the pulmonary immune responses will be discussed. The first line of defense against A. fumigatus conidia is the innate immune system.

\section{Complement}

The innate immune system contains opsonins, which are non-specific components that bind to different moieties on the surface of potential pathogens and mark them for removal by circulating phagocytes or directly induce microbial lysis. The role of complement in A. fumigatus clearance has been examined by several groups. The lectin and alternative complement pathways have been hypothesized to have functional roles in A. fumigatus clearance due to the high levels of accessible PAMPs, including glycoproteins and mannose in resting conidia. Formation of a membrane attack complex (MAC) has a minor role in the clearance of A. fumigatus conidia, possibly due to the thickness of the cell wall; however, the impact of complement, specifically 
component $\mathrm{C} 5$, on recruitment of phagocytes is thought to be an important step in antifungal immune responses $(50,51)$.

Ultimately, the amount of $\mathrm{C} 3$ that binds to the surface of a microorganism can significantly affect the binding, endocytosis, and degradation of conidia. As previously discussed, A. fumigatus particles can exist in three forms: resting conidia, swollen conidia, and hyphae, each of which are potential activators of the complement system. Studies by Kozel et al. indicate that resting conidia bind C3 molecules at a slower rate than swollen conidia or hyphae. Different complement pathways are activated based upon the fungal particle morphology (52). The alternative pathway is associated with resting conidia, while the classical pathway becomes activated upon conidial swelling and hyphal formation (52). This is likely due to the presence of anti-Apergillus antibodies that form against the antigens that become accessible following conidial swelling and hyphal formation. C3 binding is also effected by temperature, as binding is more efficient at body temperature $\left(37^{\circ} \mathrm{C}\right)$ than at lower temperatures. Furthermore, binding of C3 induces a rapid interaction of resting conidia with phagocytic cells (within 30 minutes) and enhances phagocytosis. Depletion of IgG from serum has no effect on the binding of C3 to conidial surfaces, thereby demonstrating the importance of the alternative and potentially the lectin pathways (53).

A. fumigatus has several mechanisms to prevent C3 binding, and subsequent complement activation, to the surface of conidia and subsequent. These mechanisms lend to the opportunistic pathogenicity of the fungus, as resting conidia from more pathogenic species of Aspergillus (A. flavus, A. fumigatus, A. niger, and A. terreus) tend to bind fewer C3 molecules than those less likely to cause infection (54). Factor H, along with Factor H-like protein-1 (FHL-1), and Factor H-related protein 1 (FHR-1), are negative regulators of the alternative pathway that commonly bind to vertebrate cells, thereby inhibiting the formation of $\mathrm{MAC}$ on host cells. A. fumigatus conidia strongly bind Factor H, FHL-1, and FHR-1while hyphal forms are less likely to bind these molecules (19). The C4b-binding protein (C4bp), another regulatory molecule which blocks the cascade of the classical and lectin pathways, also binds to the conidial surface (55). Through binding of these complement inhibitory molecules to the surface of A. fumigatus conidia, the fungus actively prevents further activation of the complement cascade. This can also effect the recruitment of phagocytic cells to the site of the fungus. Additionally, A. fumigatus conidia can also bind plasminogen, a serum protein that when activated to plasmin has been 
shown to have proteolytic activity against bacteria. Plasmin is important for the degradation of the proteinaceous extracellular matrix, allows for tissue invasion by microorganisms, and also degrades complement proteins that bind to the surface of conidia. By binding plasminogen, preventing its subsequent activation to plasmin, the conidia are protected from enhanced binding of complement factors (56).

Hyphae, hyphal fragments, and germinating conidia also contain other molecules capable of evading complement. Hyphae produce an alkaline protease, Alp1, which can inhibit complement binding, and can also degrade complement proteins including $\mathrm{C} 3,4$ and 5. Upon conidial swelling and germination, A. fumigatus also produces several factors, called mycotoxins, Some of these toxins are known proteases that function to degrade host-proteins involved in fungal clearance. A. fumigatus hyphal culture supernatant, containing Alp1, has been shown to cleave complement proteins C3, C4, C5, and C1q, as well as IgG (50). Further, Alp1 can also degrade CR3, a complement receptor that mediates uptake by phagocytes $(50,57)$. Although many aspects of complement interaction with A. fumigatus have been characterized, much work remains to advance the understanding of the roles, interactions, and mechanisms involved in complement/fungal immune responses.

\section{Epithelial cells}

A. fumigatus conidia first encounter with the respiratory epithelium following natural exposures. The rodlet layer is believed to bind to the extracellular matrix proteins, laminin and fibronectin. Several genes have been identified that contain putative glycophosphatidylinositol (GPI)-anchored proteins and $\mathrm{N}$-terminal signal sequences that are hypothesized to play a role in the adherence of conidia to the extracellular matrix of the respiratory epithelium. The conidial wall also contains large quantities of carbohydrates, including glucans, galactomannans, and chitin which have been shown to mediate fungal adherence (58). Each of these factors has provided insight into the initial mechanisms involved in the binding of A. fumigatus conidia to epithelial cells. Different types of epithelial cells also have different propensities to bind $A$. fumigatus conidia. Ciliated tracheobronchial epithelial cells do not bind conidia, whereas nonciliated epithelial cells internalize conidia into intracellular vesicles. Paris and colleagues also illustrated the ability of endothelial cells to endocytose A. fumigatus conidia (59). Using in vitro experiments with a primary nasal epithelial cell line, Botterel and colleagues showed that $A$. 
fumigatus conidia were rapidly engulfed by nasal epithelial cells, and trafficked to a late endosomal-lysosomal compartment within 4 hours, but were not degraded by the cell. Despite not being cleared by the nasal epithelial cells, the engulfed conidia did not germinate within 20 hours post-exposure (60). It has also been shown that in addition to A. fumigatus conidia binding extracellular matrix proteins; they also bind to A549 respiratory type II epithelial cells in a concentration dependent manner. This binding is enhanced in the presence of the proinflammatory cytokine, interferon- $\gamma$ (IFN- $\gamma$ ) (61). Lung surfactant D protein prevented binding of conidia to epithelial cells (62). In the study, A. fumigatus conidia began to germinate and penetrate a confluent epithelial layer within 12 hours (63). Once internalized, conidia are bound within acidified phagolysosomes, where a small percentage is able to survive. Of the $3 \%$ of surviving conidia, 34\% germinate within the phagolysosome by 24 hours post-infection and by 36 hours, these germlings may escape the phagolysosome, avoid host cell lysis, and form extracellular hyphae (64). Therefore, the germination reported by DeHart et al. was likely a function of germination of extracellular conidia (63).

A major mechanism for the induction of invasive disease is permeation of the endothelial layer by actively growing hyphae. During germination, A. fumigatus also produce numerous proteases which degrade the tight junctions present in the epithelial layer that aid in colonization. In a study comparing the ability of A549 cells and human umbilical endothelial cells to internalize A. fumigatus conidia, it was shown that epithelial cells (A549) internalize $30 \%$ of bound conidia, while endothelial cells are capable of engulfing $50 \%$ of conidia (65). Internalization of conidia was most efficient at $37^{\circ} \mathrm{C}$. This study indicated that endothelial cells are more efficient at internalizing conidia, which is likely a conserved characteristic used by the host as a mechanism to ensure pathogens that bypass the epithelial layer are less likely to invade the blood stream. Additionally, killed conidia were internalized at comparable levels, indicating that the interaction of conidia and epithelial or endothelial cells is through a heat-stable molecule, such as surface carbohydrates (65). Viable conidia induced injury of the endothelial cells, which was not noted in response to killed hyphae. Conversely, hyphae also induce significant endothelial cell tissue factor activity, which was not apparent in response to conidia. These results suggest that invasive infection likely arises from the ability of hyphae to invade and injure endothelial cells, as well as induce tissue factor activity through unknown mediators (66). 
It remains unclear why A. fumigatus is able to colonize the respiratory mucosa. Modulation of host cell apoptosis is a common mechanism used by pathogenic microorganisms as a means of evading the host immune response. Studies examining the impact of $A$. fumigatus conidia on the induction of epithelial apoptosis have illustrated that numerous Aspergillus species inhibit apoptosis. The inhibition of host cell apoptosis is achieved by viable conidia, as killed conidia do not induce this effect. Furthermore, culture filtrates, containing fumagillin and helvolic acid, from clinical isolates of A. fumigatus inhibited ciliary beat frequency, as well as induced damage to the respiratory epithelium in vitro (67). These results suggest that $A$. fumigatus toxins may influence the ability of the fungus to colonize in the respiratory epithelium. Secreted proteases have also been shown to induce the production of proinflammatory cytokines, namely IL-6 and IL-8, in A549 respiratory epithelial cells.(68). Further, gliotoxin has been shown to reduce the survival of infected mice, indicating enhanced fungal virulence (69).

Recently, conidia have been shown to bind to PRRs present on epithelial cells which leads to the production of proinflammatory cytokines. Specifically, activation induced the upregulation of TLR-2 and TLR-4, stimulation of the NF- $\kappa$ B pathway, as well as the release of proinflammatory cytokines, IL-1 $\beta$ and IL-6. Inhibition of TLR-2 and TLR-4 stunted the release of cytokines, which illustrated the importance of TLR recognition of conidia (70). Balloy et al. reported that respiratory epithelial cells detect A. fumigatus conidia through TLR-2 and TLR-4 and that germinating conidia are required for the production of IL-8 which occurs through the phosphatidylinositol 3-kinase, p38 MAPK, and ERK1/2 pathway. Experiments using MyD88dominant -negative transfected cells illustrated that the TLR-MyD88 pathway is not required for the production of IL-8. However, this pathway was activated by the presence of germinating conidia, and led to NF- $\mathrm{B}$ activation. Therefore, both the MyD88-dependent and MyD88independent pathways are activated by germinating conidia and are involved in IL-8 synthesis (71). These data show that fungal germination directly impacts the immune response, and begins with the respiratory epithelium, which is an area that will be addressed in this dissertation.

Though numerous studies have highlighted the potential role for airway epithelial cells in priming the initial immune response against A. fumigatus, it is still not clear to what extent these cells play in the overall immune response. Data suggests that these cells are capable of inducing a proinflammatory response against conidia. However, it appears that these responses only occur following germination of conidia. These data suggest that A. fumigatus conidia have an innate 
function that allows them to interact with epithelial cells, induce their own endocytosis, survive within acidified phagolysosomes, and thereby evade the ensuing immune response. The conidia utilize the epithelial cells for protection, and contain mechanisms that allow for germination and invasion from these cells. Further studies are required to examine any additional immune functions that epithelial cells may have, the impact of these responses on the overall immune response against A. fumigatus, as well as to establish further insights into the mechanism(s) associated with interaction of $A$. fumigatus conidia with these cells.

\section{Macrophages}

Macrophages within the lung, termed alveolar macrophages, are the first professional phagocyte to encounter inhaled particles or potential pathogens. As part of the initial innate immune response, these cells are responsible for engulfing microorganisms, killing them through several mechanisms, and producing and secreting cytokines to recruit other effector cells to the site of infection. These steps are crucial to mounting and shaping the immune response against potential pathogens, including fungi. Though there is a baseline presence of alveolar macrophages following exposure to A. fumigatus, these cells are actively recruited to the lung and peak by 72 hours (72).

Fungal particles specifically bind to alveolar macrophages through interactions of the cell wall components (PAMPs), with the PRRs on the host macrophages. Three predominant PRRs known to play a role in the recognition of A. fumigatus are TLR-2, TLR-4, and Dectin-1, though others may be involved (73). The interaction between fungi and the specific PRRs was shown to be fungal-morphology specific. TLR-4 interacts primarily with resting conidia, TLR-2 with resting and swollen conidia, while Dectin-1 recognizes exposed $\beta$-glucans, binds on each form, but more efficiently to swollen conidia and hyphae. Thus, fungal recognition by macrophages is mediated through carbohydrate, mannose, and lectin moieties present on both conidia and hyphae (74). TLR-2 activation is necessary for optimal signaling, as macrophages from TLR-2 deficient mice that were incubated with resting conidia or hyphae produced less inflammatory cytokines than control macrophages $(75,76)$. The release of TNF $\alpha$ by macrophages may also be, in part, mediated by unidentified serum components (77). These studies also illustrated the importance of the MyD88 signaling pathway in antifungal responses (76). 
Recognition of $A$. fumigatus conidia and hyphae by PRRs on host cells leads to phagocytosis. With alveolar macrophages, A. fumigatus is phagocytosed using two different types of phagocytosis: 1) pseudopod formation around conidia, or 2) zipper phagocytosis of larger hyphae. Within 3 hours, $>90 \%$ of conidia are internalized in $\mathrm{J} 774$ murine macrophages, and macrophages can contain up to 3 conidia $(76,78)$. The phagosomes rapidly fuse to early and late endosomes, leading to phagolysosome maturation and acidification within 3 hours, and $>99 \%$ conidial killing within 12 hours $(75,76,79)$. Once internalized, oxygen radicals are also rapidly produced (79). Inhibition of NADPH oxidases decreases the amount of ROS produced and inhibits conidial killing without impacting phagocytic abilities (73).

Additionally, germinating conidia, through increased interactions between Dectin-1 and the newly accessible $\beta$-glucans, have been shown to induce neutrophil recruitment to the airways and stimulate the production and secretion of TNF $\alpha / \mathrm{MIP} 2$ by alveolar macrophages, while resting conidia did not have this effect (80). TLR-2 and MyD88-mediated signals were shown to provide an additive contribution to the activation of macrophages by germinating conidia. Activation of macrophages results in the recruitment of neutrophils to the affected site. Alveolar macrophages are known to efficiently bind and engulf resting conidia; however, this interaction does not induce the production of proinflammatory cytokines. On the other hand, swollen conidia and hyphae are strong inducers of $\mathrm{NF \kappa B}$, significantly increasing the production and secretion of proinflammatory cytokines and ROS. This response is, in part, mediated by the activation of the Dectin-1 receptor (81). Dectin-1 is also able to stimulate the production of TNF $\alpha$ in the absence of TLR-2 or MyD88 signaling. Overall, these results indicate that the pulmonary immune response is directed toward metabolically active fungi, and ultimately the recognition of cell wall $\beta$-glucans by Dectin- 1 , to avoid unnecessary tissue damage that would result from the frequent inhalation of ubiquitous conidia $(81,82)$.

Hyphal exposure to macrophages stimulates the production of IL-10 through a TLR-2 dependent mechanism (76). It has also been shown that the function of TLRs is likely redundant in immunocompetent mice, as neutropenic mice with TLR-2, TLR-4, and MyD88 tripleknockouts do not survive a pulmonary A. fumigatus infection (83). In the presence of neutrophils, however, TLR-2, TLR-4, or MyD88 knockout mice are not more susceptible to invasive aspergillosis than WT mice (84). Furthermore, mice lacking functional TLR-2 and TLR-4 showed severely impaired neutrophil recruitment (85). This illustrates an essential role 
for TLR-2 and TLR-4 -mediated phagocytosis in the absence of polymorphonuclear cells (PMNs). Immunosuppression through corticosteroid administration negatively impacted macrophage function, leading to the decreased production of IL- $1 \alpha$ and TNF $\alpha$, which led to a significant decrease in IL-6 and TNF $\alpha$ production (84).

Corticosteroids, often used for immunosuppression in patients, also inhibit ROS production, production of proinflammatory cytokines, and fungal clearance by macrophages (73, 86). Importantly, these responses are species-specific to A. fumigatus and A. flavus, but not A. terreus nor A. niger (86). Some conidia are often associated with unsealed phagosomes, which likely leads to lung disease due to the rapid release of oxygen radicals (2). However, it is unknown if conidia have an innate mechanism that may prevent phagosome sealing. Additionally, gliotoxin has been shown to preferentially bind and inhibit rodent macrophagemediated phagocytosis of particulate matter, as well as induction of morphological alterations. Specifically, gliotoxin, at concentrations well below toxic levels, was able to affect glucose metabolism, macromolecular synthesis, and inhibit bactericidal activity in macrophages (87-89).

Further, immunocompetent, naïve, Dectin-1-deficient mice are more sensitive to an intratracheal challenge with A. fumigatus than control mice, and exhibit greater than $80 \%$ mortality within 5 days of exposure. Alveolar macrophages from Dectin-1 knockout mice showed impaired fungal uptake and failed to produce proinflammatory cytokines and chemokines. This resulted in insufficient recruitment of neutrophils and uncontrolled fungal growth, as neutrophils primarily respond to germinated fungi (73). The requirement for dectin-1 signaling has also been shown in vivo. Dectin-1 activation, through interactions with swollen conidia or hyphae triggers the greatest level of proinflammatory cytokine and chemokine production, i.e. TNF $\alpha$, IL- $1 \alpha$ and $\beta$, IL-6, CXCL2/MIP2, CCL3, MIP1a, KC, GCSF, and GMCSF. This resulted in the impaired cellular recruitment, and increase in fungal burden, which illustrates the importance of the $\beta$-glucan/Dectin- 1 interaction (80).

Different A. fumigatus particles also influence the production of the inflammatory response in different types of macrophages. Different types of macrophages, as identified through surface markers, are also known to differentially respond to A. fumigatus conidia. Classical monocytes $\left(\mathrm{CD} 14^{+} \mathrm{CD} 16^{-}\right)$inhibit conidial germination, yet secrete minimal $\mathrm{TNF} \alpha$, while $\mathrm{CD}_{1} 4^{+} \mathrm{CD} 16^{+}$monocytes do not inhibit germination, but secrete large amounts of TNF $\alpha$ (7). Additionally, it has been shown that following infection, alveolar macrophages rapidly 
express Arginase-1, which is a key marker used to identify alternatively activated macrophages (AAMs). Induction of AAMs was not dependent on Dectin-1 or MyD88 signaling, and was shown to have a protective role in the anti-fungal response (72). Overall, these studies have shown that depletion of macrophages in a murine model significantly increased fungal burden, highlighting the importance of alveolar macrophages in the innate immune response against $A$. fumigatus (72).

Further studies have indicated that A. fumigatus can inhibit phagocytosis and phagolysosome acidification, and is able to escape the macrophage. A common immune evasion strategy used by pathogens is inhibition of host cell apoptosis. Swollen A. fumigatus conidia have been shown to inhibit macrophage apoptosis through inhibition of caspase-3, an important enzyme in the cell death pathway, though the mechanism for this is unknown (6). Other studies indicate that, upon intracellular formation of hyphae, alveolar macrophages may not be lysed by the fungus. Rather, the hyphae continue to elongate, and surpass the alveolar cell wall barrier without inducing cell death, as a potential means of minimizing the inflammatory response that ensues following cell lysis.

Overall, these studies illustrate an importance for TLR-mediated macrophage recognition of A. fumigatus which results in proinflammatory cytokine production. These cytokines are responsible for recruitment of neutrophils, which is necessary for clearance of germinating conidia. These studies encompass both in vitro and in vivo studies. In the in vivo studies, aspiration or instillation exposures were conducted, which ignore the potential alteration in responses that may occur following exposures to dry conidia as opposed to fungal suspensions. This is an aspect that will be addressed in Chapters 3 and 4 of this dissertation.

\section{Neutrophils}

Prolonged neutropenia is the predominant immune deficiency associated with the induction of invasive aspergillosis, highlighting the importance of this cell population in host defense against $A$. fumigatus. In contrast to alveolar macrophage depletion, which can be functionally compensated during fungal clearance, early recruitment of neutrophils is essential for defense against A. fumigatus. Neutrophils are recruited to the airways following exposure and their numbers peak between 24 and 48 hours post-exposure (72). Depletion of neutrophils within 3 hours of exposure is associated with high mortality $(90,91)$. This implies that neutrophils, and 
not necessarily alveolar macrophages, may be essential in host defense against A. fumigatus conidia. However, redundant mechanisms of alveolar macrophages and neutrophils may act synergistically to aid each other and enhance antifungal responses. Alveolar macrophages phagocytose and kill conidia, but not hyphae, whereas neutrophils are capable of mounting an anti-fungal response against both forms of A. fumigatus.

Once recruited, neutrophils respond by phagocytosing conidia and hyphae, through mechanisms similar to those used by alveolar macrophages. Neutrophil-mediated fungal clearance occurs through 3 classical neutrophil responses (34). Upon phagocytosis of either fungal conidia or hyphae, neutrophils immediately begin producing ROS and phagolysosome acidification. Neutrophil ROS production is dependent upon signaling through the dectin-1 receptor, as neutrophils of dectin-1 knockout mice have impaired ROS production (92). Additionally, neutrophils can undergo degranulation upon interaction with microbes. This involves the release of numerous antimicrobial compounds from cytoplasmic granules, including alkaline phosphatases, lysozyme, lactoferrin, defensins, serine proteases, and many others. These compounds function to kill extracellular microbes, and mice lacking one of these compounds exhibit decreased A. fumigatus killing. Importantly, these killing mechanisms are morphotype specific as resting conidia do not significantly induce neutrophil degranulation or respiratory bursts (93). A third method of neutrophil mediated killing is the release of neutrophil extracellular traps (NETs).

NETs are web-like extracellular DNA fibers that contain antimicrobial compounds, such as serine proteases (94). Dying neutrophils release their DNA in an effort to trap extracellular microorganisms, while the antimicrobials within the NETs, as well as those secreted from other nearby neutrophils, degrade the organism. The formation of NETs in response to A. fumigatus is a relatively new finding; therefore, the intricacies of this mechanism are actively being examined. However, human neutrophils have been shown to produce NETs in vitro and in vivo in response to A. fumigatus (94). NET formation is a mechanism used by the host to capture invading pathogens, and thus is maximal in response to A. fumigatus hyphal formation, though they are still produced, to a lesser extent, in response to resting and swollen conidia. Importantly, presence of RodA inhibits NET formation, which explains maximal NET formation against hyphae which do not have a rodlet layer. While NETs are decorated with fungicidal proteins, it has been shown that NETs do not play a major role in fungal killing. However, it has been 
hypothesized that the main function of NET formation is to prevent further spreading of germinating fungi (94).

Though neutrophils contain multiple mechanisms for fungal clearance, the production of ROS species is the predominant killing mechanism. For example, individuals with chronic granulomatous disease (CGD) have a mutation in the gene encoding NADPH oxidase, and therefore, do not produce ROS (95). These individuals are unable to effectively clear $A$. fumigatus and are susceptible to infection. A study examining the transcriptional profile of $A$. fumigatus conidia following incubation with neutrophils from CGD or healthy patients showed that there were increases in the mRNA levels of genes involved in degradation of fatty acids, peroxisome formation, and the glyoxylate cycle. These data suggest that conidia alter their metabolism to adjust to the host environment. Further, mRNA levels of genes encoding proteins involved in iron/copper assimilation were higher in normal neutrophils exposed to conidia than CGD neutrophils (95). Another study reported acute sensitivity to invasive disease in oxidasedeficient humans and mice (96). The authors reported that neutrophils were recruited to the lungs rapidly following infection, and responded by phagocytosing conidia and facilitating extracellular killing of germinated hyphae through ROS dependent mechanisms (96). In addition, following pulmonary instillation of $A$. fumigatus conidia in BALB/c mice, neutrophils were recruited to the lung and formed functioning oxidase aggregates with conidia, which inhibited germination (97). In CXCR2 $2^{-/}$, involved in neutrophil recruitment, or gp91 ${ }^{\text {phox }}$-/- a NADPH oxidase knockout model, mice had delayed neutrophil recruitment to the lung and increased germination in comparison to the BALB/c mice at 6 and 12 hours post exposure (97). In the gp91 ${ }^{\text {phox }}$-/- mice, germination was extensive in neutrophils, yet negligible in alveolar macrophages. BALB/c mice also had increased peribronchiolar neutrophil accumulation, but no germination, while the $\mathrm{CXCR}^{-/}$mice contained germinating conidia at 6 hours, which was cleared by 48 hours, and also recruited fewer inflammatory cells. In contrast, gp $91^{\text {phox }-/-}$ mice had extensive hyphal formation by 6 hours which resulted in tissue invasion by 48 hours. This study suggests that, in addition to activity by alveolar macrophages, neutrophil recruitment and the production of ROS and oxidative-active aggregates are essential in preventing A. fumigatus conidial germination (97).

While neutrophils are essential in the clearance of $A$. fumigatus, uncontrolled activity can lead to host damage and disease (98). Neutrophils are capable of contributing to allergic 
phenotypes (99). Antibody-mediated neutrophil depletion following A. fumigatus exposures in a murine model displayed reduced AHR and airway remodeling. Further, transgenic overexpression of the neutrophil chemotactic molecule CXCL1 resulted in increased airway neutrophil recruitment but not other leukocytes, and worsened allergic responses. Depletion of neutrophils in this transgenic model alleviated the severity of allergy. Overall, these data suggests that neutrophil responses against fungi are tightly regulated, and that in some instances, neutrophils may actually contribute to fungal allergic diseases (99).

Furthermore, neutrophils are resistant to gliotoxin-induced apoptosis (87). This is another potential mechanism used by the fungus to enhance survival and germination, due to restriction of immune cells that function to control tissue inflammation, thereby allowing the unaffected neutrophils to continually produce inflammatory mediators that lead to tissue destruction (87). Neutrophil-mediated phagocytosis can be blocked by gliotoxin due to the induction of significant actin cytoskeleton reorganization, which results in cell shrinkage and the loss of filapodia required for phagocytosis. Gliotoxin can also inhibit ROS production in neutrophils (100). In summary, gliotoxin can negatively affect the phagocytic and clearance abilities of circulating neutrophils, allowing for dissemination of A. fumigatus (101).

Due to the requisite role for neutrophils in the efficient clearance of A. fumigatus conidia and hyphae, it is necessary to examine the role of these cells in shaping the overall immune responses following exposure in an immunocompetent animal model. Because most studies examine A. fumigatus pathogenesis in neutropenic mouse models of invasive infection, there is limited in vivo data available on the role neutrophils in the pulmonary immune responses. This will be addressed in the following chapters of this dissertation.

\section{Eosinophils}

Eosinophils are granulocytic cells that combat parasitic and viral infections (98). The granules within these cells contain numerous antimicrobial components, including eicosanoids, ROS, major basic protein, and enzymes including RNases, etc. Upon activation of eosinophils within the affected tissue, and subsequent phagocytosis of a microorganism, eosinophils undergo degranulation and release the contents of the granules. Eosinophils also degrade the engulfed organism using mechanisms similar to macrophages and neutrophils (98). Further, eosinophil cell numbers are increased in allergy. Despite the link between repeated fungal exposures and the 
induction of allergy, asthma, or ABPA, little information is available on the interactions of eosinophils by A. fumigatus. However, Bhatia et al. have shown that eosinophils are recruited to the lungs within 24 hours, and remain elevated until 72 hours post exposure (72). Increased eosinophil numbers remain for up to 2 weeks after exposure, though continuous presence of IL-5 and IL-13 can lead to eosinophil persistence (72).

It has been shown that murine eosinophils are required for the induction of an allergendependent $\mathrm{T}_{\mathrm{H}} 2$ pulmonary immune response which is mediated by DCs and $\mathrm{T}$ cells (102). Recruitment of peripheral eosinophils to the lung-draining lymph nodes is required for accumulation of myeloid DCs, which induces antigen-specific $\mathrm{T}$ effector cell production. This process did not require the expression of major histocompatibility complex II (MHC II) on eosinophils (102). This data suggests that eosinophils have an accessory role, as opposed to direct $\mathrm{T}$ cell interactions, in the activation of antigen-specific $\mathrm{T}$ cells. Further, eosinophils are also able to uniquely suppress $\mathrm{DC}$-mediated production of $\mathrm{T}_{\mathrm{H}} 17$ cells, and to a lesser extent $\mathrm{T}_{\mathrm{H}} 1$ responses, thereby promoting $\mathrm{T}_{\mathrm{H}} 2$ polarization and the induction of A. fumigatus allergic responses (103).

Investigators have also examined the impact of fungal cell wall components on the immune response. To this end, the ability of chitin, a cell wall component associated with $\beta$ glucans, to induce eosinophil-mediated allergy has been examined (102). Cell wall preparations from Aspergillus that had been isolated from house dust samples from homes of asthmatic individuals, was able to induce a heightened recruitment of eosinophils into the murine lung. This was blunted by the enzymatic degradation of chitin and $\beta$-glucans. The expression of chitinase in murine lungs resulted in significantly lower eosinophil recruitment following fungal exposure. Blocking the activity of the chitinase resulted in prolonged duration of eosinophilia in the lungs. These results suggest that chitin, derived from household environments associated with asthmatic patients, is capable of inducing eosinophilic allergic inflammation within the lungs, though exacerbation of this response may be potentially be impacted by the coexistence of $\beta$-glucans (102).

Though the presence of eosinophils within the lungs of A. fumigatus exposed animals has been documented, it remains unknown if the route of exposure impacts eosinophil levels and the induction of allergy/asthma. In the studies presented in Chapters 2 and 3, two different exposure routes are utilized and levels of eosinophils are examined. 


\section{Natural killer cells}

Natural killer (NK) cells are innate immune cells with cytolytic functions, similar to $\mathrm{T}$ cells of the adaptive immune system. The hallmark feature of these cells is the native ability to recognize and respond to 'non-self' cells, as well as tumor or infected cells, without the prerequisite for priming and classical activation (98). NK cells do not require the genetic reassembly of surface receptors, as do T and B cells. Instead, the surface receptors for NK cells are a mixture of germ-line encoded receptors that signal an activation or inhibition status. A balance between these signals dictates the status and response of the cell, though these mechanisms are not fully understood (104). When NK cells encounter foreign cells, they secrete enzymes, such as granzymes and perforin, which attack the cell membranes of microorganisms and induce lysis. These cells are also known to produce large amounts of IFN- $\gamma$, activate macrophages, induce the up-regulation of MHC I on professional antigen presenting cells, and also have anti-proliferative effects on malignant and virally infected cells (104). Thus, NK cells play an important role in the induction of $\mathrm{T}_{\mathrm{H}} 1$ responses, which are important for the clearance of fungal conidia (104). Recent evidence indicates that NK cells may also develop a memory response against commonly encountered antigens, and may aid in shaping the overall adaptive immune response against invading pathogens (105).

There have been few reports on the role of NK cells on A. fumigatus pulmonary clearance. In an invasive aspergillosis murine model with neutropenia, MCP-1/CCL2 is upregulated in the lungs, which leads to the recruitment of NK cells (106). NK cells have also been shown to be the predominant cell type responsible for the production of high levels of IFN- $\gamma$ (107). When MCP-1/CCL2 or antibody-mediated NK cells were neutralized, early NK cell recruitment was abrogated, yet there was no effect on the recruitment of other leukocytes. The inhibition of NK cell recruitment led to twice the mortality and greater than 3 times the fungal burden in the lungs of infected mice, which was independent of $\mathrm{T}$ and $\mathrm{B}$ cell activity $(106,107)$. These results indicated that NK cells are a major source of IFN- $\gamma$ during early A. fumigatus infections, and therefore play a critical role in the early host defense against A. fumigatus (106, 107).

Due to the ability of NK cells to kill tumor and infected cells, there has been an increased interest in the adoptive transfer of these cells into hematopoetic stem cell transplant patients as a therapy to combat infection (108). Because stem cell transplant patients comprise a large 
population of patients that are highly susceptible to invasive aspergillosis, studies examined the feasibility of adoptive transfer of NK cells. The authors reported that both un-stimulated and IL2 pre-stimulated NK cells were able to effectively kill $A$. fumigatus hyphae, but had no effect on resting conidia. NK cell-secreted supernatant and perforin were also able to kill A. fumigatus. Interestingly, the high levels of IFN- $\gamma$ and granulocyte, macrophage-colony stimulating factor (GM-CSF) produced by activated NK cells were sequestered by A. fumigatus, indicating the immunosuppressive capabilities of the fungus. Due to the ability of NK cells to specifically kill hyphae, these results suggested that adoptive transfer of these cells into stem cell recipients may be beneficial in the context of invasive aspergillosis (108).

\section{Dendritic cells}

DCs are the professional antigen presenting cell of the immune system, and are therefore responsible for shaping the overall adaptive immune response against invading pathogens. These cells are located just beneath the epithelial layer of the respiratory tract, and constantly monitor the lumen for potential pathogens. DCs recognize pathogens through PRR interactions similar to macrophages and neutrophils, by binding to microbial-specific PAMPs. Once activated, DCs internalize and degrade the pathogens into peptides. These peptides are loaded onto MHC molecules and trafficked to the surface of the cell. Once activated, the lectin receptor CCR7, is up-regulated on the surface of the DC which results in homing to different lymphoid organs where antigen presentation occurs. Interactions between the MHC molecules and co-receptors on the $\mathrm{DC}$ with $\mathrm{T}$ and/or $\mathrm{B}$ cell receptors and co-receptors activate the adaptive cells for effector function and clonal expansion (98).

Fungal $\beta$-glucans are known to induce and enhance the maturation of DCs (109). Interaction of yeast glucan particles with bone marrow derived DCs (BMDCs) stimulated the secretion of TNF $\alpha$, which was mediated through dectin-1. Activation of TLR-9 or TLR-2/1 with yeast glucan particles and TLR-specific ligands resulted in higher levels of secreted IL-10, and IL-1 $\alpha$ while decreasing the levels of IL-1 $\beta$, IL-6, IFN- $\gamma$-inducible-protein-10 (109). These results suggest that different fungal morphotypes have the capacity to alter the immune responses in DCs, and therefore, alter the ensuing immune response against fungi (110). Furthermore, viable A. fumigatus conidia induce monocyte-derived immature DC death within 6 hours. This coincided with germ tube formation, and resulted in the differential regulation of several fungal 
genes including the up-regulation of genes involved in fermentation, drug transport, pathogenesis, Asp f 1 allergen, and responses to oxidative stress (111). This interaction also resulted in the up-regulation of DC genes involved in proinflammatory cytokine production. Overall, these data show that, in addition to the up-regulation of proinflammatory cytokines induced in A. fumigatus following incubation with macrophages and neutrophils, the fungus also specifically adapts to phagocytosis by DCs that enhance its survival (111).

The interactions between DCs and A. fumigatus conidia or hyphae allows for internalization of A. fumigatus through phagocytosis and recognition receptors $(112,113)$. Pathogens commonly gain access through the aforementioned TLRs, as well as dendritic cellspecific ICAM-3 grabbing non-integrin (DC-SIGN). DC-SIGN has been shown to specifically interact with clinical isolates of A. fumigatus, and may be important in the initial interactions between the fungus and the host immune system (114). DC phagocytosis occurs through different mechanisms, coiling phagocytosis for conidia and zipper phagocytosis for hyphae, which involves the cooperation of distinct fungal recognition receptors. Optimal DC phagocytosis occurs between 60-120 minutes following exposure and is dependent on opsonization. In contrast, hyphae are rapidly degraded within 2 hours following internalization by DCs. Importantly, gliotoxin production by hyphae can specifically bind to DCs which inhibits function and induces apoptosis, which directly affects antigen presentation and the formation of adaptive immune responses (87-89).

Recognition of different fungal morphotypes leads to the production of different cytokine responses. Upon incubation with A. fumigatus, immature myeloid DCs produce IL-12p70 and IL-4 (111). Internalization of conidia by DCs results in production of proinflammatory cytokines, predominantly IL-12p70 (111). In contrast, DCs internalizing hyphae produce larger amounts of the $\mathrm{T}_{\mathrm{H}} 2$ cytokine, IL-4, while producing no IL-12. Furthermore, DCs are unable to kill conidia although conidial antigens are still processed and presented to $\mathrm{T}$ cells in the draining lymph nodes (112). Thus, conidial-bearing DCs induce the priming of $\mathrm{T}_{\mathrm{H}} 1 \mathrm{CD} 4 \mathrm{~T}$ cells that produce large amounts of IFN- $\gamma$ (115). DCs home to the draining lymph nodes or the spleen, following ingestion of A. fumigatus, through interaction of CCR7 on DCs and its ligands CCL19 and CCL21 (116). Due to the general inflammatory nature of fungal-activated DCs, this process can be detrimental to the tissue during the infectious process. It has been shown that CCR7 deficiency in pulmonary DCs leads to the production of TNF $\alpha$, CXCL-10, and CXCL-2, leading 
to enhanced activation of these cells. Thus, in invasive aspergillosis models, adoptive transfer of CCR7 deficient DCs resulted in increased numbers of inflammatory DCs, leading to increased survival and less pulmonary injury (116).

Invasive aspergillosis is associated with the accumulation of inflammatory DCs within the lungs which has been shown to be protective (117). In a murine model of invasive aspergillosis, challenge with killed or attenuated A. fumigatus conidia resulted in the enhanced accumulation of inflammatory, but not other subsets of DCs in neutropenic mice (117). This effect was not observed in non-neutropenic mice. These results suggest that the infiltration of inflammatory DCs was due to a mechanism independent of neutrophil-mediated killing, and likely due to the enhanced influx of these cells to the lung due to high levels of TNF expression in neutropenic mice. High levels of TNF expression led to a greater lung expression of DC recruiting ligand, CCL2 and CCL20 (117). Furthermore, plasmacytoid DCs (pDCs), a subtype of DCs known to produce large amounts of type I IFN, spread over hyphae and inhibit further invasive growth (118). This antifungal activity is due to secreted substances and does not require direct cell-fungus interactions. The fungus combats this response by secreting cytotoxic substances in an attempt to kill the pDCs. During this time, the pDCs release high concentrations of proinflammatory cytokines via TLR-9 independent mechanisms. These cells play a role in the anti- $A$. fumigatus immune responses, as large numbers of pDCs are recruited to the lung following pulmonary exposure to hyphae. However, in vivo depletion of pDCs led to increased invasive aspergillosis susceptibility (92). Furthermore, deletion of monocyte-derived DCs reduces Th1 and enhances Th17, which leads to decreased clearance of fungal conidia (119).

In humans, DCs obtained from healthy volunteers have the capacity to phagocytose heat killed A. fumigatus conidia, which results in the up-regulation of maturation markers, including HLA molecules as well as the coreceptors CD80 and CD86 (120). These activated DCs display increased production of IL-12. DCs stimulated ex vivo with A. fumigatus antigen were also able to stimulate lymphocyte proliferation and the increased production of IFN- $\gamma$ (120). Furthermore, the stimulated DCs were able to partially restore the antifungal capacity of lymphocytes following adoptive transfer (121). These results were further supported by another group that showed adoptive transfer of fungal-activated DCs can impact the outcome of infection, as these cells can activate protective, non-protective, and $\mathrm{T}_{\text {Reg }}$ cells (122). Overall, these results suggest 
that adoptive transfer of ex vivo stimulated DCs may be a useful therapy for combating invasive infection in neutropenic hosts.

\section{A. fumigatus-Induced Adaptive Immunity}

The adaptive immune response does not result in the rapid removal of foreign pathogens, due to the time required (7-10 days following initial exposure) for adaptive cells to be primed, activated, expand, and be recruited to the site of infection (98). $\mathrm{T}$ and $\mathrm{B}$ cells are the primary cell types of the adaptive immune response. APCs from the site of infection are homed to the secondary lymphoid tissues after ingestion and processing of microbes. At this site, APCs bind, through surface MHC-peptide complexes and co-receptors, to predetermined antigen-specific $\mathrm{T}$ or $\mathrm{B}$ cell receptors, leading to the activation and clonal expansion of the adaptive cells. These cells are then recruited to the site of infection where the cytokine environment controls which type of response the naïve cells will mount and which antibodies will be secreted. Thus, the innate response against the invading pathogen directly affects the type, level, and effectiveness of the adaptive response.

T cells

There are several types of $\mathrm{T}$ cells that play a role in the clearance or pathogenesis of $A$. fumigatus exposures. In general, CD4 effector cells with a $\mathrm{T}_{\mathrm{H}} 1$ phenotype, consisting of TNF $\alpha$, IFN- $\gamma$, IL-12, and IL-1 $\beta$, are required for the effective clearance of A. fumigatus conidia, and subsequent resistance to invasive infection $(34,123,124)$. Susceptibility to invasive infection is associated with a decreased production of these cytokines, with an increase in IL-4 and IL-10, which are classical $\mathrm{T}_{\mathrm{H}} 2$ and $\mathrm{T}_{\mathrm{Reg}}$ cytokines, respectively (123). In WT mice, pulmonary infection via i.t. challenge with $A$. fumigatus induces $\mathrm{T}_{\mathrm{H}} 1, \mathrm{~T}_{\mathrm{H}} 2$, and $\mathrm{T}_{\mathrm{H}} 17$ responses simultaneously (110). Dectin- 1 signaling in these mice also reduces IFN- $\gamma$ and IL-12p40 production in the lungs, which leads to a decrease in T-bet expression, a $\mathrm{T}_{\mathrm{H}} 1$ transcription factor, in responding CD4 $\mathrm{T}$ cells. However, dectin-1 knockout mice disproportionately increase $T_{H} 1$ responses and decrease $T_{H} 17$ differentiation. Responding CD4 $\mathrm{T}$ cells, in the absence of IFN- $\gamma$, IL-12p35, or T-bet, increase $\mathrm{T}_{\mathrm{H}} 17$ differentiation independent of Dectin-1 signaling (110). Furthermore, IL-17 production within the lungs is dependent on dectin-1, which is important because neutralization of IL-17 can impair fungal clearance (92). 
As humans are constantly exposed to fungi, it is important to understand the mechanisms that mediate $\mathrm{T}$ cell responses following repeated exposures, and how these result in clearance/protection vs. infection vs. allergy/asthma induction. In a C57BL/6 murine model of repeated intranasal A. fumigatus exposures, greater than $99 \%$ of conidia were cleared within 24 hours. Importantly, repeated exposures did not lead to the accumulation of conidia or germination over time (125). A secondary exposure resulted in the early recruitment of neutrophils and $\mathrm{T}_{\text {Regs }}$ into the lungs, though overall inflammation was minimal. Repeated exposures also resulted in the continual expansion of $\mathrm{T}$ cells within the draining lymph nodes. By four exposures, eosinophils and myeloid cell influx to the lungs peaked, and goblet cell hyperplasia/mucus production and fibrosis was observed throughout the response, and $\mathrm{T}_{\mathrm{H}} 2$ cytokines were produced. Repeated exposures, up to 8 , led to $\mathrm{T}$ cell activation and the development of $\mathrm{T}_{\mathrm{H}} 1, \mathrm{~T}_{\mathrm{H}} 2$, and $\mathrm{T}_{\mathrm{H}} 17$ responses. Importantly, the numbers of CD4 $\mathrm{T}$ cells and $\mathrm{T}_{\text {Regs }}$ did not affect the number of myeloid cells, which implies that CD4 $\mathrm{T}$ cells may function to reduce the innate inflammatory responses that are chronically induced following repeated exposures (125).

While IL-17 expression is classically thought to be proinflammatory, and results in the recruitment of neutrophils to the area of infiltration, $\mathrm{T}_{\mathrm{H}} 17$ responses have been shown in the induction/exacerbation of fungal allergy (126). $\mathrm{T}_{\mathrm{H}} 17$ cells can differentiate in the absence of proinflammatory cytokines. However, in IL-17 deficient mice repeatedly exposed to $A$. fumigatus conidia, inflammation was reduced, predominantly affecting the influx of eosinophils as IL-17 is central in the extravasation of eosinophils from the blood (103). In these mice, conidial clearance was also enhanced, while $\mathrm{T}_{\text {Reg }}$ induction was dampened. Thus, these results indicate that IL-17 may be partly responsible for driving $\mathrm{T}_{\mathrm{H}} 2$ responses following repeated exposures (103).

CD4 $\mathrm{T}$ cells also have the ability to develop into memory cells, which has important implications in developing better immuno-therapeutics. In a murine model of invasive aspergillosis, mice that survived the primary infection contained IFN- $\gamma$ producing CD4 T cells. These mice were also resistant to subsequent infection (127). Mice that did not survive the infection had mounted an immune response that was characterized by the production of IL-4 by CD4 T cells, and had impaired antifungal neutrophil responses. Interestingly, when these mice were treated with soluble IL-4 receptor, greater than $70 \%$ of mice did not succumb to infection 
and were also protected from a secondary A. fumigatus infection. Therefore, CD4 IL-4producing $\mathrm{T}$ cells play a key role in determining susceptibility to invasive A. fumigatus infections (127). A. fumigatus-specific CD4 $\mathrm{T}_{\mathrm{H}} 1$ cells also have the ability to confer cross-protection against Candida albicans due to the presence of a highly conserved immunogenic epitope from the A. fumigatus cell wall glucanase called Crfl (128). This epitope can be presented by three common MHC II alleles, and induces a group of memory CD4 $\mathrm{T}$ cells that are cross-reactive with $C$. albicans. Because A. fumigatus and $C$. albicans are the two predominant fungal species associated with invasive infection in immunocompromised individuals, this information may be useful for developing cross-protective immunotherapies (128).

The concentration of conidia that are inhaled during exposures directly influences the type of immune response. In a murine model of $A$. fumigatus, exposure to fewer than $2 \times 10^{3} \mathrm{~A}$. fumigatus conidia, which is more than 3 logs less than the aforementioned exposure studies, leads to the generation of a $\mathrm{T}_{\mathrm{H}} 2$ response and eosinophilia, hallmarks of allergic lung disease (129). Conversely, exposures to higher concentrations of $A$. fumigatus conidia $\left(2 \times 10^{5}\right)$ induce a neutrophil-mediated response that resulted in a $\mathrm{T}_{\mathrm{H}} 1$ cell predominant, proinflammatory response (129). IL-17A was produced despite the concentration of conidia used for exposures, illustrating the requirement for IL-17A in both inducing and controlling the inflammatory response. Notably, A. fumigatus conidial exposures led to invasive infection, even at the lowest concentration, in $\mathrm{Rag}^{-/-}$mice lacking mature $\mathrm{T}$ and $\mathrm{B}$ cells. In contrast, B cell deficient mice cleared conidia in a manner similar to WT mice, which indicates a role for $\mathrm{T}$ cells in protection against fungal dissemination (129).

Adaptive immune responses against A. fumigatus have predominantly focused on CD4 T cells, while CD8 T cells have been largely ignored. CD8 T cells are the classical effector T cells that respond to tumor cells and viral infections. Experiments with ovalbumin transgenic (OT) mice showed that sensitization of OT-I (CD8 T cells) with ovalbumin and A. fumigatus extracts promoted clonal expansion and IFN- $\gamma$ producing cytotoxic cells, suggesting that A. fumigatus may have an adjuvant effect on production of effector $T$ cells (130). Furthermore, experiments from our lab showed that repeated aspiration exposures using viable A. fumigatus conidia induced IFN- $\gamma$ producing CD8 T cells (131). This population of cells was decreased in mice exposed to heat-killed or fixed A. fumigatus conidia, as well as A. versicolor, which is not able to germinate at $37^{\circ} \mathrm{C}$. Furthermore, viable A. fumigatus conidia persisted within the lungs longer 
than heat-killed or A. versicolor conidia. This correlated to increased maintenance of airway memory CD8 T cells (131). Overall, the precise role of CD8 T cells in A. fumigatus-mediated immune responses is unknown, though it is likely that these cells are critical in the induction of memory responses.

Furthermore, due to the gliotoxin-induced apoptosis of macrophages and DCs, the $A$. fumigatus-specific CD4 $\mathrm{T}$ cell responses are also suppressed $(87,88)$. This toxin can also induce apoptosis in splenocytes, thymocytes, and mesenteric lymph node cells. Further, gliotoxin can selectively deplete mature lymphocytes within the bone marrow. It also specifically inhibits NFkB DNA binding in $\mathrm{T}$ and $\mathrm{B}$ cells, and has been shown to suppress functional $\mathrm{T}$ cell responses, leading to overall immunosuppression (132). Additionally, pretreatment of a mixed lymphocyte culture with gliotoxin results in decreased induction of cytotoxic $\mathrm{T}$ cells, even at low concentrations (87). In conclusion, toxins produced by A. fumigatus are extremely capable of negatively affecting each cell type involved in fungal clearance, and are a major factor that impacts the host-immune response against the fungus.

Though much evidence on the role of CD4 T cells in A. fumigatus immune responses is available, there is limited information about the role of CD8 $\mathrm{T}$ cells. Furthermore, these responses are based on unnatural aspiration exposures in both immune-suppressed and competent mouse models. Therefore, it remains unknown if the route of exposure to dry vs. wet conidia affects $\mathrm{T}$ cell populations and cytokine production. To address these caveats, CD4 and CD8 $\mathrm{T}$ cell populations were analyzed following repeated aspiration or nose-only dry aerosol inhalation and will be presented in Chapters 2 and 3 of this dissertation.

B cells

Little is known about the role of $\mathrm{B}$ cells in anti-A. fumigatus adaptive immune responses. Healthy individuals have been shown to produce A. fumigatus-specific antibodies, including IgM and IgG (26). Those with fungal induced allergy and/or asthma have also been shown to produce IgE antibodies. Further, IgA antibodies have been identified in invasive aspergillosis patients, and to a lesser extent, asthmatics that tested positive to an A. fumigatus antigen skin-prick test (133). Passive administration of fungal-specific antibodies enhances fungal clearance. However, B cell deficient mice that effectively cleared a primary infection did not elicit a protective memory response during secondary infection. These results indicate that B-cell antibody 
production play a role in the anti-fungal immune responses, though the mechanisms associated with fungal-induced antibody class switching and production, as well as the importance of B cells in the long-term immune response to repeated exposures remains largely unknown. Furthermore, identification of the fungal-specific immuno-dominant proteins/peptides that are responsible for specific-B cell activation and expansion has yet to be elucidated. This information would aid in developing improved diagnostics and immunotherapeutic options.

There is limited information available on the recruitment of $\mathrm{B}$ cells to the lungs following exposures to A. fumigatus. Furthermore, little is known about A. fumigatus-specific B cell responses in the lymph nodes, including fungal factors that affect the clonal expansion, classswitching, and antibody production, and the impact of B cells on developing allergy. To address these unknowns, studies presented in Chapter 3 of this dissertation examined the presence of B cells within the lungs following multiple dry, A. fumigatus conidia exposures. Furthermore, B cells were also examined in mediastinal lymph nodes, and assessed for IgE production.

\section{A. fumigatus Melanins}

Melanins are heterogeneous group of phenolic compounds that form large, macromolecular pigments that are extensively intercalated throughout the conidial wall (12). These pigments are responsible for the characteristic blue-green color of WT A. fumigatus conidia. As fungi are predominantly environmental microorganisms and plant pathogens, melanin functions to protect the conidia from ultraviolet light and ensures the integrity of conidia under the stress of turgor pressure $(11,12,134)$. Within a mammalian host, however, melanin has several different functions which protect fungi from the host immune system $(8,134)$. As a result, melanin has been classified as a fungal virulence factor.

Using melanin knock-downs and albino mutants, melanins have been shown to enhance conidial survival by quenching reactive oxygen species (ROS), and prevent binding of complement protein C3 to the surface of the conidia (135-139). Melanin also appears to protect the conidia from the innate immune system by preventing phagolysosome acidification and inhibition of host-cell apoptosis $(77,140,141)$. Conidia from melanin mutants also exhibit decreased virulence in a mouse model of invasive aspergillosis $(135,136,142)$. Additionally, the presence of melanin in A. fumigatus conidia has been shown to attenuate the host proinflammatory cytokine response of human peripheral blood mononuclear cells, as the conidia of 
the albino mutant induce higher levels of IL-6, TNF- $\alpha$, and IL-10 than wild-type conidia (143). Similar results have been shown with low melanin producing mutants of $C$. neoformans, as these conidia induce greater inflammatory responses and are cleared more rapidly, as well as induce greater $\mathrm{CD}^{+} \mathrm{T}$ cell responses and TNF- $\alpha$ levels. Furthermore, albino conidia are more susceptible to host-mediated killing, as these conidia are more rapidly degraded than WT (135).

Though fungal melanins have been shown to influence the immune response against $A$. fumigatus in immunosuppressed mice, there is no evidence that demonstrates the impact of melanin following repeated exposures in competent mice and the role it may play in allergy induction. Since persistence of an antigen is one factor that leads to the induction of allergic responses, it was hypothesized that melanin may be an important element in the induction of fungal allergies. This hypothesis will be addressed by this dissertation.

\section{Animal models}

Numerous animal models have been used to examine the impact of A. fumigatus exposures in vivo. In these models, common methods of exposure include: 1) exposure of animals using aerosol chambers, 2) intratracheal instillation, and 3) pharyngeal/intranasal aspirations to conidial suspensions and/or fungal extracts (reviewed in Templeton et al (144)). These methods have been employed for mouse, rat, rabbit, and guinea pig exposures. Although a multitude of available data has been obtained from animal models of A. fumigatus exposures, the information derived from these studies is confounded for several important reasons. Most models of exposure examine the pathogenicity of A. fumigatus in immunocompromised animals and focus predominantly on infection. To date, little is known about the A. fumigatus-mediated immune mechanisms associated with the induction of allergy and asthma. Most importantly, the data acquired from those studies may not be accurate, as the exposure models used do not reflect natural exposures to A. fumigatus.

In many of these exposure methods, suspension of fungal conidia within saline is used. Fungal conidia contain multiple surface antigens. Upon suspension in saline, these surface antigens may be washed off and diluted. Dilution or removal of these surface antigens may alter the interaction of the conidia with immune cells, and in doing so, alter the ensuing immune response. Further, as was previously noted, an environment with high water content is often sufficient for activation of the fungal conidia. Initially, the conidia would begin to swell, which 
would alter its surface structure and expose numerous PAMPs that had previously been hidden in the cell wall of a resting conidium. Accessibility of these different PAMPs by immune cells would result in the activation of different cell types, which could significantly alter the cytokine environment, the recruitment of inflammatory cells and lymphocytes, and could ultimately, impact the type of response that the host mounts against the fungus. Activation causes a change in conidial metabolism, which results in the production of several enzymes and proteins that are known to hinder the immune response against fungi $(3,34)$. Importantly, a small molecular weight $(<10 \mathrm{kDa})$, heat-stable toxin has been shown to be released from conidia of environmental and clinical isolates within minutes of liquid suspension. This toxin is distinct from hyphal toxins, and has been shown to decrease ROS production by macrophages (145). Since many of the immune mechanisms known to play a role in fungal immunity may be impacted by approaches using liquid extracts, it is vital to determine the impact of dry fungal aerosols on the pulmonary immune responses to fully understand how natural fungal exposures may impact these areas.

Another caveat associated with A. fumigatus exposure studies is the lack of information using animals that have been repeatedly exposed. In many studies, immunocompromised animals are given a single conidial dose to model invasive aspergillosis. While invasive aspergillosis is a disease that remains the focus of the research community, the majority of the population is not immunocompromised. Those studies that use immunocompetent animals, commonly expose naïve animals a single time, and then sacrifice the animal to examine multiple endpoints. Therefore, the impact of repeated A. fumigatus exposures in immunocompetent models is under studied. As humans are exposed to approximately $10^{4}$ conidia $/ \mathrm{m}^{3}$ daily, with the potential to be exposed to larger concentrations in occupational settings, it is of interest to determine what type of immune response occurs following repeated exposures and how the concentration of conidia may affect these results (2).

Studies of the influence of dry fungal exposures on the immune response are limited. To date, one group has attempted to address the role of dry fungal exposures in murine model. Schuh et al. developed a dry-fungal aerosol exposure chamber and examined immune responses in mice that had previously been primed to induce allergy. In this model, mice were immunized, both subcutaneously and intraperitoneally, a single time with a commercial A. fumigatus fungal extract containing a heterogenous mixture of proteins from both conidia and hyphae using alum 
as an adjuvant. Mice were then exposed to the extract, intranasally, once per week for three weeks. Following these exposures, mice were either exposed a single time to a suspension of $5 \mathrm{x}$ $10^{6}$ conidia via intratracheal instillation, or were subjected to inhalation exposure up to three times at different intervals following the first inhalation exposure. Aerosols were created by directing a stream of air across a fungal culture plate. The nose-only 'chamber', which accommodated 3 mice total, was pre-coated with fungi for 10 minutes prior to actual exposures. A sample of the aerosol was examined under oil immersion, and the authors noted that this sample did not contain swollen conidia or hyphae. The inhalation exposures required mice to be anesthetized and subjected to nose-only exposures for ten minutes. The total number of conidia that the mice were exposed to was quantified post-exposure. These studies reported multiple differences, including an increase in the eosinophils and lymphocytes, antibody responses, and overall lung inflammation to dry-aerosols when compared to liquid involuntary aspiration. The authors also noted that dry inhalation exposures exacerbated the allergic asthma phenotype.

The Schuh et al. studies suggested that dry conidial exposures induced different, heightened immune responses following repeated exposures, thereby further demonstrating the overall importance of this project (146). However, there were several limitations associated with their model that will be addressed by this dissertation. In the forthcoming studies, using an acoustical generator dry aerosol exposure system, 1) the number of fungal particles aerosolized, 2) of the appropriate size (2-5 $\mu \mathrm{m}$ in diameter), and 3) being deposited in the lungs can be monitored in real-time. This allows for the determination of the length of the exposure based on the desired lung deposition concentration, a function that was not addressed using the Schuh exposure system (146). the Pitt-3 acoustical generator nose-only chamber allows for the exposure of 15 mice at a single time. The fungal conidia used for exposure are grown on rice grains, a natural substrate, not on a fungal enrichment medium as was done in the Schuh studies, which may significantly alter their protein profile. The growth of fungi on rice was previously developed by Sorenson et al. as a method that enables improved aerosolization of fungal conidia (147). In our nose-only unit, aerosols are composed of predominantly single conidia but not hyphal fragments and other fungal aggregates. Furthermore, the animals are not anesthetized during the exposures, so that their respiratory volume, depth of breath, can be quantified and is not affected by anesthetization. 


\section{OVERVIEW}

In Chapter 2, mice were repeatedly exposed to A. fumigatus conidia using pharyngeal aspiration to characterize the pulmonary immune responses following exposures in immunocompetent animals. Further, the impact of fungal melanization in these responses was also examined. Three different A. fumigatus strains from a clinical isolate were utilized in these studies: 1) A. fumigatus wild-type (WT) conidia or one of two different strains with mutations in the melanin production pathway 2) $\Delta a r p 2$ (tan), or 3) $\Delta a l b 1$ (white). Through these experiments, it was determined that the presence of melanin impacts the neutrophil and eosinophil responses, yet does not induce detectable specific antibody production following exposures. Further, as was previously shown in an invasive infection immunocompromised mouse model, the presence of melanin inhibits the clearance of the conidia. Most importantly, it is shown that the presence of melanin impacts the accumulation of $\mathrm{Tc} 17$ cells, a cell type that had not been previously associated with fungal exposures, yet may play an important role in the clearance of conidia.

In Chapter 3, a nose-only exposure chamber is used to examine the impact of dry conidia on the murine immune response following repeated exposures. In these studies, the wild-type and melanin mutant conidia were used for exposures. Repeated exposures to each strain induced similar levels of inflammation, which was also comparable to the data presented in Chapter 2. Mice that were exposed to melanized fungi (WT) showed delayed airwayreactivity (AR) at 48 hours post-exposure, while the $\Delta$ albl mutant AR was higher at 24 hours post-exposure. Furthermore, in agreement with experiments from Chapter 2, melanized fungal conidia were more resistant to clearance, as a higher number of conidia are present at early time-points following the final exposure when compared to $\triangle a l b 1$ conidia. Cellularity data from these studies show that exposures to dry conidia result in a mixed Th1/Th2 response in both the lungs and mediastinal lymph nodes. The presence of melanin also resulted in higher levels of Tc17 cells over the time course of the experiment. Sera isolated from exposed mice also showed immunoglobulin reactivity to conidial extracts; however, each exposure group exhibited reactivity to different conidial antigens, despite a similar protein profile between the species.

The final chapter of this dissertation will discuss the impact of the data on delineating several aspects of A. fumigatus exposures that have been previously overlooked using alternate exposure methods Through the use of melanin mutant conidia, the data presented here indicates that while melanin is an important A. fumigatus virulence factor in immunocompromised mouse 
models, it does not appear to play a major role in the induction of an allergic phenotype in a mouse model of repeated exposures. Importantly, these are the first studies to show that Tc17 cells are present and affected by the presence of conidial melanin and germination. Further, the utilization of a nose-only dry fungal exposure chamber allowed for collection of data that more accurately represents, in comparison to the data reported following exposure to fungal suspensions, the immune response that occurs following natural exposures to A. fumigatus conidia. Finally, sera from exposed mice led to identifying the $\operatorname{IgG}$ dominant components of $A$. fumigatus that may be important for fungal pathogenesis. The identification of these proteins may be used in the future to develop more reliable immunodiagnostic tests, as target for antifungal therapies, or as potential components that may be used to develop vaccines to protect against A. fumigatus infections. 


\section{REFERENCES}

1. Curtis, L., A. Lieberman, M. Stark, W. Rea, and M. Vetter. 2004. Adverse health effects of indoor molds. Journal of Nutritional \& Environmental Medicine 14: 261-274.

2. Eduard, W. 2009. Fungal spores: a critical review of the toxicological and epidemiological evidence as a basis for occupational exposure limit setting. Critical Reviews in Toxicology 39: 799-864.

3. Latgé, J.-P., and W. J. Steinbach. 2009. Aspergillus fumigatus And Aspergillosis. ASM Press.

4. Paris, S., J.-P. Debeaupuis, R. Crameri, M. Carey, F. Charlès, M. C. Prévost, C. Schmitt, B. Philippe, and J. P. Latgé. 2003. Conidial Hydrophobins of Aspergillus fumigatus. Applied and Environmental Microbiology 69: 1581-1588.

5. Aimanianda, V., J. Bayry, S. Bozza, O. Kniemeyer, K. Perruccio, S. R. Elluru, C. Clavaud, S. Paris, A. A. Brakhage, S. V. Kaveri, L. Romani, and J.-P. Latge. 2009. Surface hydrophobin prevents immune recognition of airborne fungal spores. Nature 460: 1117-1121.

6. Aimanianda, V., and J.-P. Latgé. 2010. Fungal hydrophobins form a sheath preventing immune recognition of airborne conidia. Virulence 1: 185-187.

7. Pihet, M., P. Vandeputte, G. Tronchin, G. Renier, P. Saulnier, S. Georgeault, R. Mallet, D. Chabasse, F. Symoens, and J.-P. Bouchara. 2009. Melanin is an essential component for the integrity of the cell wall of Aspergillus fumigatus conidia. BMC Microbiology 9: 177.

8. Butler, M. J., A. W. Day, J. M. Henson, and N. P. Money. 2001. Pathogenic Properties of Fungal Melanins. Mycologia 93: 1-8.

9. Hamilton, A. J., and B. L. Gomez. 2002. Melanins in fungal pathogens. Journal of Medical Microbiology 51: 189-191.

10. Henson, J., M. Butler, and A. Day. 1999. THE DARK SIDE OF THE MYCELIUM: Melanins of Phytopathogenic Fungi. Annual Review of Phytopathology 37: 447-471.

11. Jacobson, E. S. 2000. Pathogenic roles for fungal melanins. Clinical Microbiology Reviews 13: 708-717.

12. Wheeler, M. H., and A. A. Bell. 1988. Melanins and their importance in pathogenic fungi. Current Topics in Medical Mycology 2: 338-387.

13. Abad, A., J. Fernndez-Molina, J. Bikandi, A. Ramrez, J. Margareto, J. Sendino, F. Hernando, J. Pontn, J. Garaizar, and A. Rementeria. 2010. What makes Aspergillus fumigatus a successful pathogen? Genes and molecules involved in invasive aspergillosis. Revista iberoamericana de micolog ̃̃a 27: 155-182.

14. van de Veerdonk, F. L., B. J. Kullberg, J. W. M. van der Meer, N. A. R. Gow, and M. G. Netea. 2008. Host-microbe interactions: innate pattern recognition of fungal pathogens. Current Opinion in Microbiology 11:305-312.

15. Chai, L. Y. A., A. G. Vonk, B. J. Kullberg, P. E. Verweij, I. Verschueren, J. W. M. van der Meer, L. A. B. Joosten, J.-P. Latgé, and M. G. Netea. 2011. Aspergillus fumigatus cell wall components differentially modulate host TLR2 and TLR4 responses. Microbes and Infection 13: 151-159.

16. Ayerst, G. 1969. The effects of moisture and temperature on growth and spore germination in some fungi. Journal of Stored Products Research 5: 127-141. 
17. Nanguy, S. P.-M., J.-M. Perrier-Cornet, M. Bensoussan, and P. Dantigny. 2010. Impact of water activity of diverse media on spore germination of Aspergillus and Penicillium species. International Journal of Food Microbiology 142: 273-276.

18. Cramer, R. A., A. Rivera, and T. M. Hohl. 2011. Immune responses against Aspergillus fumigatus: what have we learned? Current Opinion in Infectious Diseases 24: 315-322 310.1097/QCO.1090b1013e328348b328159.

19. Brakhage, A., S. Bruns, A. Thywissen, P. Zipfel, and J. Behnsen. 2010. Interaction of phagocytes with filamentous fungi. Current opinion in microbiology 13: 409-415.

20. Shwab, E. K., and N. P. Keller. 2008. Regulation of secondary metabolite production in filamentous ascomycetes. Mycological Research 112: 225-230.

21. Tomee, J. F., and H. F. Kauffman. 2000. Putative virulence factors of Aspergillus fumigatus. Clinical and Experimental Allergy 30: 476-484.

22. Shen, H.-D., M. F. Tam, R.-B. Tang, and H. Chou. 2007. Aspergillus and Penicillium allergens: focus on proteases. Current Allergy and Asthma Reports 7: 351-356.

23. Kupfahl, C., A. Michalka, C. Lass-Flörl, G. Fischer, G. Haase, T. Ruppert, G. Geginat, and H. Hof. 2008. Gliotoxin production by clinical and environmental Aspergillus fumigatus strains. International Journal of Medical Microbiology 298: 319-327.

24. Bozza, S., C. Clavaud, G. Giovannini, T. Fontaine, A. Beauvais, J. Sarfati, C. D'Angelo, K. Perruccio, P. Bonifazi, S. Zagarella, S. Moretti, F. Bistoni, J.-P. Latg, and L. Romani. 2009. Immune sensing of Aspergillus fumigatus proteins, glycolipids, and polysaccharides and the impact on Th immunity and vaccination. The Journal of Immunology 183: 2407-2414.

25. Simon-Nobbe, B., U. Denk, V. Pöll, R. Rid, and M. Breitenbach. 2008. The spectrum of fungal allergy. International Archives of Allergy and Immunology 145: 58-86.

26. Chaudhary, N., J. Staab, and K. Marr. 2010. Healthy human T-Cell responses to Aspergillus fumigatus antigens. PLoS ONE 5: e9036-e9036.

27. Green, B. J., J. K. Sercombe, and E. R. Tovey. 2005. Fungal fragments and undocumented conidia function as new aeroallergen sources. The Journal of Allergy and Clinical Immunology 115: 1043-1048.

28. Green, B. J., E. R. Tovey, J. K. Sercombe, F. M. Blachere, D. H. Beezhold, and D. Schmechel. 2006. Airborne fungal fragments and allergenicity. Medical Mycology 44 Suppl 1: S245-255.

29. Hogaboam, C. M., K. J. Carpenter, J. M. Schuh, and K. F. Buckland. 2005. Aspergillus and asthma--any link? Medical Mycology 43 Suppl 1: S197-202.

30. Lin, S. J., J. Schranz, and S. M. Teutsch. 2001. Aspergillosis case-fatality rate: systematic review of the literature. Clinical infectious diseases 32: 358-366.

31. Groll, A. H., M. Kurz, W. Schneider, V. Witt, H. Schmidt, M. Schneider, and D. Schwabe. 1999. Five-year-survey of invasive aspergillosis in a paediatric cancer centre. Epidemiology, management and long-term survival. Mycoses 42: 431-442.

32. Tong, K. B., C. J. Lau, K. Murtagh, A. J. Layton, and R. Seifeldin. 2009. The economic impact of aspergillosis: analysis of hospital expenditures across patient subgroups. International journal of infectious diseases : IJID : official publication of the International Society for Infectious Diseases 13: 24-36.

33. Latge, J. P. 2001. The pathobiology of Aspergillus fumigatus. Trends in Microbiology 9: 382-389. 
34. Latgé, J. P. 1999. Aspergillus fumigatus and aspergillosis. Clinical Microbiology Reviews 12: 310-350.

35. Zmeili, O. S., and A. O. Soubani. 2007. Pulmonary aspergillosis: a clinical update. QJM 100: 317-334.

36. Kan, V. L., M. A. Judson, V. A. Morrison, S. Dummer, D. W. Denning, J. E. Bennett, T. J. Walsh, T. F. Patterson, and G. A. Pankey. 2000. Practice guidelines for diseases caused by Aspergillus. Clinical Infectious Diseases 30: 696-709.

37. Al-Alawi, A., Ryan, CF, Flint, JD, Mueller, NL. 2005. Aspergillus-related lung disease. Canadian Respiratory Journal 12: 377-387.

38. Devereux, G. 2006. The increase in the prevalence of asthma and allergy: food for thought. Nat Rev Immunol 6: 869-874.

39. Tariq, S. M., S. M. Matthews, M. Stevens, and E. A. Hakim. 1996. Sensitization to Alternaria and Cladosporium by the age of 4 years. Clinical \& Experimental Allergy 26: 794-798.

40. Chaudhary, N., and K. Marr. 2011. Impact of Aspergillus fumigatus in allergic airway diseases. Clinical and Translational Allergy 1: 4.

41. Agarwal, R., A. N. Aggarwal, D. Gupta, and S. K. Jindal. 2009. Aspergillus hypersensitivity and allergic bronchopulmonary aspergillosis in patients with bronchial asthma: systematic review and meta-analysis The International Journal of Tuberculosis and Lung Disease 13: 936-944.

42. Corey, J. P., S. Kaiseruddin, and A. Gungor. 1997. Prevalence of mold-specific immunoglobulins in a Midwestern allergy practice.

43. Lopez, M., and J. Salvaggio. 1985. Mold-sensitive asthma. Clin Rev Allergy 3: 183-196.

44. Hsieh, K.-H., and J.-J. Shen. 1988. Prevalence of childhood asthma in Taipei, Taiwan, and other Asian Pacific countries. Journal of Asthma 25:73-82.

45. Gioulekas, D., A. Damialis, D. Papakosta, F. Spieksma, P. Giouleka, and D. Pataka. 2004. Allergenic fungi spore records (15 years) and sensitization in patients with respiratory allergy in Thessaloniki-Greece. Journal of Investigational Allergology and Clinical Immunology 14.

46. Patterson, K., and M. E. Strek. 2010. Allergic Bronchopulmonary Aspergillosis. Proceedings of the American Thoracic Society 7: 237-244.

47. Selman, M., Y. Lacasse, A. Pardo, and Y. Cormier. 2010. Hypersensitivity Pneumonitis Caused by Fungi. Proceedings of the American Thoracic Society 7: 229-236.

48. Lacasse, Y., and Y. Cormier. 2006. Hypersensitivity pneumonitis. Orphanet Journal of Rare Diseases 1:25.

49. Wright, J. R. 2005. Immunoregulatory functions of surfactant proteins. Nat Rev Immunol 5: 58-68.

50. Behnsen, J., F. Lessing, S. Schindler, D. Wartenberg, I. Jacobsen, M. Thoen, P. Zipfel, and A. Brakhage. 2010. Secreted Aspergillus fumigatus protease Alp1 degrades human complement proteins C3, C4, and C5. Infection and immunity 78: 3585-3594.

51. Waldorf, A. R., and R. D. Diamond. 1985. Neutrophil chemotactic responses induced by fresh and swollen Rhizopus oryzae spores and Aspergillus fumigatus conidia. Infection and Immunity 48: 458-463.

52. Kozel, T. R., M. A. Wilson, T. P. Farrell, and S. M. Levitz. 1989. Activation of C3 and binding to Aspergillus fumigatus conidia and hyphae. Infection and immunity 57: 34123417. 
53. Sturtevant, J., and J.-P. Latgé. 1992. Participation of complement in the phagocytosis of the conidia of Aspergillus fumigatus by human polymorphonuclear cells. Journal of Infectious Diseases 166: 580-586.

54. Henwick, S., S. V. Hetherington, and C. C. Patrick. 1993. Complement binding to Aspergillus conidia correlates with pathogenicity. The Journal of laboratory and clinical medicine 122: 27-35.

55. Vogl, G., I. Lesiak, D. B. Jensen, S. Perkhofer, R. Eck, C. Speth, C. Lass-Flörl, P. F. Zipfel, A. M. Blom, M. P. Dierich, and R. Würzner. 2008. Immune evasion by acquisition of complement inhibitors: The mould Aspergillus binds both factor $\mathrm{H}$ and C4b binding protein. Molecular Immunology 45: 1485-1493.

56. Behnsen, J., A. Hartmann, J. Schmaler, A. Gehrke, A. A. Brakhage, and P. F. Zipfel. 2008. The opportunistic human pathogenic fungus Aspergillus fumigatus evades the host complement system. Infect. Immun. 76: 820-827.

57. Rambach, G., D. Dum, I. Mohsenipour, M. Hagleitner, R. Würzner, C. Lass-Flörl, and C. Speth. 2010. Secretion of a fungal protease represents a complement evasion mechanism in cerebral aspergillosis. Molecular Immunology 47: 1438-1449.

58. Sheppard, D. C. 2011. Molecular mechanism of Aspergillus fumigatus adherence to host constituents. Current Opinion in Microbiology 14: 375-379.

59. Paris, S., E. Boisvieux-Ulrich, B. Crestani, O. Houcine, D. Taramelli, L. Lombardi, and J. P. Latgé. 1997. Internalization of Aspergillus fumigatus conidia by epithelial and endothelial cells. Infection and immunity 65: 1510-1514.

60. Botterel, F., K. Gross, O. Ibrahim-Granet, K. Khoufache, V. Escabasse, A. Coste, C. Cordonnier, E. Escudier, and S. Bretagne. 2008. Phagocytosis of Aspergillus fumigatus conidia by primary nasal epithelial cells in vitro. BMC Microbiology 8: 97.

61. Bromley, I., and K. Donaldson. 1996. Binding of Aspergillus fumigatus spores to lung epithelial cells and basement membrane proteins: relevance to the asthmatic lung. Thorax 51: 1203-1209.

62. Yang, Z., S. M. Jaeckisch, and C. G. Mitchell. 2000. Enhanced binding of Aspergillus fumigatus spores to A549 epithelial cells and extracellular matrix proteins by a component from the spore surface and inhibition by rat lung lavage fluid. Thorax 55: 579-584.

63. DeHart, D. J., D. E. Agwu, N. C. Julian, and R. G. Washburn. 1997. Binding and Germination of Aspergillus fumigatus Conidia on Cultured A549 Pneumocytes. Journal of Infectious Diseases 175: 146-150.

64. Wasylnka, J. A., and M. M. Moore. 2003. Aspergillus fumigatus conidia survive and germinate in acidic organelles of A549 epithelial cells. Journal of Cell Science 116: 1579-1587.

65. Wasylnka, J. A., and M. M. Moore. 2002. Uptake of Aspergillus fumigatus conidia by phagocytic and nonphagocytic cells in vitro: quantitation using strains expressing green fluorescent protein. Infection and Immunity 70: 3156-3163.

66. Bezerra, L. M. L., and S. G. Filler. 2004. Interactions of Aspergillus fumigatus with endothelial cells: internalization, injury, and stimulation of tissue factor activity. Blood 103: 2143-2149.

67. Amitani, R., G. Taylor, E. Elezis, C. Llewellyn-Jones, J. Mitchell, F. Kuze, P. Cole, and R. Wilson. 1995. Purification and characterization of factors produced by Aspergillus 
fumigatus which affect human ciliated respiratory epithelium. Infect. Immun. 63: 32663271.

68. Borger, P., G. H. Koëter, J. A. Timmerman, E. Vellenga, J. F. Tomee, and H. F. Kauffman. 1999. Proteases from Aspergillus fumigatus induce interleukin (IL)-6 and IL-8 production in airway epithelial cell lines by transcriptional mechanisms. The Journal of Infectious Diseases 180: 1267-1274.

69. Watanabe, A., K. Kamei, T. Sekine, M. Waku, K. Nishimura, M. Miyaji, and T. Kuriyama. 2003. Immunosuppressive substances in Aspergillus fumigatus culture filtrate. Journal of Infection and Chemotherapy 9:114-121.

70. Zhao, J., and X.-Y. Wu. 2008. Aspergillus fumigatus antigens activate immortalized human corneal epithelial cells via Toll-Like Receptors 2 and 4. Current Eye Research 33: 447-454.

71. Balloy, V., J.-M. Sallenave, Y. Wu, L. Touqui, J.-P. Latg, M. Si Tahar, and M. Chignard. 2008. Aspergillus fumigatus-induced interleukin- 8 synthesis by respiratory epithelial cells is controlled by the phosphatidylinositol 3-kinase, p38 MAPK, and ERK1/2 pathways and not by the toll-like receptor-MyD88 pathway. Journal of biological chemistry 283: 30513-30521.

72. Bhatia, S., M. Fei, M. Yarlagadda, Z. Qi, S. Akira, S. Saijo, Y. Iwakura, N. van Rooijen, G. Gibson, C. St Croix, A. Ray, and P. Ray. 2011. Rapid host defense against Aspergillus fumigatus involves alveolar macrophages with a predominance of alternatively activated phenotype. PLOS ONE 6: e15943-e15943.

73. Philippe, B., O. Ibrahim-Granet, M. C. Prévost, M. A. Gougerot-Pocidalo, M. Sanchez Perez, A. Van der Meeren, and J. P. Latgé. 2003. Killing of Aspergillus fumigatus by alveolar macrophages is mediated by reactive oxidant intermediates. Infection and Immunity 71: 3034-3042.

74. Kan, V. L., and J. E. Bennett. 1988. Lectin-Like Attachment Sites on Murine Pulmonary Alveolar Macrophages Bind Aspergillus fumigatus Conidia. Journal of Infectious Diseases 158: 407-414.

75. Ibrahim-Granet, O., B. Philippe, H. Boleti, E. Boisvieux-Ulrich, D. Grenet, M. Stern, and J. P. Latge. 2003. Phagocytosis and intracellular fate of Aspergillus fumigatus conidia in alveolar macrophages. Infect. Immun. 71:891-903.

76. Wasylnka, J. A., A. H. T. Hissen, A. N. C. Wan, and M. M. Moore. 2005. Intracellular and extracellular growth of Aspergillus fumigatus. Medical Mycology 43: 27-30.

77. Volling, K., A. A. Brakhage, and H. P. Saluz. 2007. Apoptosis inhibition of alveolar macrophages upon interaction with conidia of Aspergillus fumigatus. FEMS Microbiology Letters 275: 250-254.

78. Loeffler, J., Z. Haddad, M. Bonin, N. Romeike, M. Mezger, U. Schumacher, M. Kapp, F. Gebhardt, G.-U. Grigoleit, S. Stevanović, H. Einsele, and H. Hebart. 2009. Interaction analyses of human monocytes co-cultured with different forms of Aspergillus fumigatus. Journal of Medical Microbiology 58: 49-58.

79. Nessa, K., L. Palmberg, U. Johard, P. Malmberg, C. Jarstrand, and P. Camner. 1997. Reaction of human alveolar macrophages to exposure to Aspergillus fumigatus and inert particles. Environmental research 75: 141-148.

80. Steele, C., R. R. Rapaka, A. Metz, S. M. Pop, D. L. Williams, S. Gordon, J. K. Kolls, and G. D. Brown. 2005. The Beta-Glucan Receptor Dectin-1 Recognizes Specific Morphologies of Aspergillus fumigatus. PLoS Pathog 1: e42. 
81. Gersuk, G. M., D. M. Underhill, L. Zhu, and K. A. Marr. 2006. Dectin-1 and TLRs permit macrophages to distinguish between different Aspergillus fumigatus cellular states. The Journal of Immunology 176: 3717-3724.

82. Luther, K., A. Torosantucci, A. A. Brakhage, J. Heesemann, and F. Ebel. 2007. Phagocytosis of Aspergillus fumigatus conidia by murine macrophages involves recognition by the dectin-1 beta-glucan receptor and Toll-like receptor 2. Cellular Microbiology 9: 368-381.

83. Cortez, K., C. Lyman, S. Kottilil, H. Kim, E. Roilides, J. Yang, B. Fullmer, R. Lempicki, and T. Walsh. 2006. Functional genomics of innate host defense molecules in normal human monocytes in response to Aspergillus fumigatus. Infection and immunity 74: 23532365.

84. Dubourdeau, M., R. Athman, V. Balloy, M. Huerre, M. Chignard, D. Philpott, J.-P. Latg, and O. Ibrahim-Granet. 2006. Aspergillus fumigatus induces innate immune responses in alveolar macrophages through the MAPK pathway independently of TLR2 and TLR4. The Journal of Immunology 177: 3994-4001.

85. Meier, A., C. J. Kirschning, T. Nikolaus, H. Wagner, J. Heesemann, and F. Ebel. 2003. Toll-like receptor (TLR) 2 and TLR4 are essential for Aspergillus-induced activation of murine macrophages. Cellular Microbiology 5: 561-570.

86. Taramelli, D., M. G. Malabarba, G. Sala, N. Basilico, and G. Cocuzza. 1996. Production of cytokines by alveolar and peritoneal macrophages stimulated by Aspergillus fumigatus conidia or hyphae. Medical Mycology 34: 49-56.

87. Müllbacher, A., and R. D. Eichner. 1984. Immunosuppression in vitro by a metabolite of a human pathogenic fungus. Proceedings of the National Academy of Sciences 81: 38353837.

88. Orciuolo, E., M. Stanzani, M. Canestraro, S. Galimberti, G. Carulli, R. Lewis, M. Petrini, and K. V. Komanduri. 2007. Effects of Aspergillus fumigatus gliotoxin and methylprednisolone on human neutrophils: implications for the pathogenesis of invasive aspergillosis. Journal of Leukocyte Biology 82: 839-848.

89. Stanzani, M., E. Orciuolo, R. Lewis, D. P. Kontoyiannis, S. L. R. Martins, L. S. St. John, and K. V. Komanduri. 2005. Aspergillus fumigatus suppresses the human cellular immune response via gliotoxin-mediated apoptosis of monocytes. Blood 105: 2258-2265.

90. Mircescu, M., L. Lipuma, N. van Rooijen, E. Pamer, and T. Hohl. 2009. Essential role for neutrophils but not alveolar macrophages at early time points following Aspergillus fumigatus infection. The Journal of Infectious Diseases 200: 647-656.

91. Ibrahim-Granet, O., G. Jouvion, T. Hohl, S. Droin-Bergere, F. Philippart, O. Kim, M. Adib-Conquy, R. Schwendener, J.-M. Cavaillon, and M. Brock. 2010. In vivo bioluminescence imaging and histopathopathologic analysis reveal distinct roles for resident and recruited immune effector cells in defense against invasive aspergillosis. BMC Microbiology 10: 105.

92. Werner, J. L., A. E. Metz, D. Horn, T. R. Schoeb, M. M. Hewitt, L. M. Schwiebert, I. Faro-Trindade, G. D. Brown, and C. Steele. 2009. Requisite role for the dectin-1 $\beta$-glucan receptor in pulmonary defense against Aspergillus fumigatus. The Journal of Immunology 182: 4938-4946.

93. Levitz, S. M., and R. D. Diamond. 1985. Mechanisms of Resistance of Aspergillus fumigatus Conidia to Killing by Neutrophils in Vitro. Journal of Infectious Diseases 152: $33-42$. 
94. Bruns, S., O. Kniemeyer, M. Hasenberg, V. Aimanianda, S. Nietzsche, A. Thywißen, A. Jeron, J.-P. Latgé, A. A. Brakhage, and M. Gunzer. 2010. Production of extracellular traps against Aspergillus fumigatus in vitro and in infected lung tissue Is dependent on invading neutrophils and influenced by hydrophobin RodA. PLoS Pathog 6: e1000873.

95. Sugui, J. A., H. S. Kim, K. A. Zarember, Y. C. Chang, J. I. Gallin, W. C. Nierman, and K. J. Kwon-Chung. 2008. Genes Differentially Expressed in Conidia and Hyphae of Aspergillus fumigatus upon Exposure to Human Neutrophils. PLoS ONE 3: e2655.

96. Boyle, K. B., D. Gyori, A. Sindrilaru, K. Scharffetter-Kochanek, P. R. Taylor, A. Mócsai, L. R. Stephens, and P. T. Hawkins. 2011. Class IA Phosphoinositide 3-Kinase $\beta$ and $\delta$ Regulate Neutrophil Oxidase Activation in Response to Aspergillus fumigatus Hyphae. The Journal of Immunology 186: 2978-2989.

97. Bonnett, C., E. J. Cornish, A. Harmsen, and J. Burritt. 2006. Early neutrophil recruitment and aggregation in the murine lung inhibit germination of Aspergillus fumigatus conidia. Infection and Immunity 74: 6528-6539.

98. Murphy, K., P. Travers, M. Walport, and C. Janeway. 2012. Janeway's immunobiology. Garland Science, New York.

99. Park, S. J., M. T. Wiekowski, S. A. Lira, and B. Mehrad. 2006. Neutrophils regulate airway responses in a model of fungal allergic airways disease. The Journal of Immunology 176: 2538-2545.

100. Eichner, R. D., M. Al Salami, P. R. Wood, and A. Müllbacher. 1986. The effect of gliotoxin upon macrophage function. International Journal of Immunopharmacology 8: 789-797.

101. Coméra, C., K. André, J. Laffitte, X. Collet, P. Galtier, and I. Maridonneau-Parini. 2007. Gliotoxin from Aspergillus fumigatus affects phagocytosis and the organization of the actin cytoskeleton by distinct signalling pathways in human neutrophils. Microbes and Infection 9: 47-54.

102. Jacobsen, E. A., K. R. Zellner, D. Colbert, N. A. Lee, and J. J. Lee. 2011. Eosinophils Regulate Dendritic Cells and Th2 Pulmonary Immune Responses following Allergen Provocation. The Journal of Immunology 187: 6059-6068.

103. Murdock, B. J., N. R. Falkowski, A. B. Shreiner, A. A. Sadighi Akha, R. A. McDonald, E. S. White, G. B. Toews, and G. B. Huffnagle. 2012. Interleukin-17 Drives Pulmonary Eosinophilia following Repeated Exposure to Aspergillus fumigatus Conidia. Infection and immunity 80: 1424-1436.

104. Caligiuri, M. A. 2008. Human natural killer cells. Blood 112: 461-469.

105. Pyzik, M., and S. M. Vidal. 2009. NK cells stroll down the memory lane. Immunol Cell Biol 87: 261-263.

106. Morrison, B., S. Park, J. Mooney, and B. Mehrad. 2003. Chemokine-mediated recruitment of $\mathrm{NK}$ cells is a critical host defense mechanism in invasive aspergillosis. Journal of Clinical Investigation 112: 1862-1870.

107. Park, S. J., M. A. Hughes, M. Burdick, R. M. Strieter, and B. Mehrad. 2009. Early NK cell-derived IFN- $\gamma$ is essential to host defense in neutropenic invasive aspergillosis. The Journal of Immunology 182: 4306-4312.

108. Schmidt, S., L. Tramsen, M. Hanisch, J.-P. Latgé, S. Huenecke, U. Koehl, and T. Lehrnbecher. 2011. Human natural killer cells exhibit direct activity against Aspergillus fumigatus hyphae, but not against resting conidia. Journal of Infectious Diseases 203: 430-435. 
109. Kikuchi, T., N. Ohno, and T. Ohno. 2002. Maturation of dendritic cells induced by Candida $\beta$-d-glucan. International Immunopharmacology 2: 1503-1508.

110. Huang, H., G. R. Ostroff, C. K. Lee, J. P. Wang, C. A. Specht, and S. M. Levitz. 2009. Distinct patterns of dendritic cell cytokine release stimulated by fungal $\beta$-glucans and toll-like receptor agonists. Infection and Immunity 77: 1774-1781.

111. Morton, C. O., J. J. Varga, A. Hornbach, M. Mezger, H. Sennefelder, S. Kneitz, O. Kurzai, S. Krappmann, H. Einsele, W. C. Nierman, T. R. Rogers, and J. Loeffler. 2011. The temporal dynamics of differential gene expression in Aspergillus fumigatus interacting with human immature dendritic cells in vitro. PLOS ONE 6: e16016.

112. Chignard, M., V. Balloy, J.-M. Sallenave, and M. Si-Tahar. 2007. Role of toll-like receptors in lung innate defense against invasive aspergillosis. Distinct impact in immunocompetent and immunocompromized hosts. Clinical Immunology 124: 238-243.

113. Wang, J. E., A. Warris, E. A. Ellingsen, P. F. Jorgensen, T. H. Flo, T. Espevik, R. Solberg, P. E. Verweij, and A. O. Aasen. 2001. Involvement of CD14 and toll-like receptors in activation of human monocytes by Aspergillus fumigatus hyphae. Infect. Immun. 69: 2402-2406.

114. Serrano-Gómez, D., A. Domínguez-Soto, J. Ancochea, J. A. Jimenez-Heffernan, J. A. Leal, and A. L. Corbí. 2004. Dendritic Cell-Specific Intercellular Adhesion Molecule 3Grabbing Nonintegrin Mediates Binding and Internalization of Aspergillus fumigatus Conidia by Dendritic Cells and Macrophages. The Journal of Immunology 173: 56355643.

115. Mambula, S. S., K. Sau, P. Henneke, D. T. Golenbock, and S. M. Levitz. 2002. Toll-like receptor (TLR) signaling in response to Aspergillus fumigatus. The Journal of Biological Chemistry 277: 39320-39326.

116. Hartigan, A. J., J. Westwick, G. Jarai, and C. M. Hogaboam. 2009. CCR7 Deficiency on Dendritic Cells Enhances Fungal Clearance in a Murine Model of Pulmonary Invasive Aspergillosis. The Journal of Immunology 183: 5171-5179.

117. Park, S. J., M. D. Burdick, W. K. Brix, M. H. Stoler, D. S. Askew, R. M. Strieter, and B. Mehrad. 2010. Neutropenia enhances lung dendritic cell recruitment in response to Aspergillus via a cytokine-to-chemokine amplification loop. The Journal of Immunology 185: 6190-6197.

118. Ramirez-Ortiz, Zaida G., Chrono K. Lee, Jennifer P. Wang, L. Boon, Charles A. Specht, and Stuart M. Levitz. 2011. A nonredundant role for plasmacytoid dendritic cells in host defense against the human fungal pathogen Aspergillus fumigatus. Cell host \& microbe 9: 415-424.

119. Rivera, A., T. Hohl, N. Collins, I. Leiner, A. Gallegos, S. Saijo, J. Coward, Y. Iwakura, and E. Pamer. 2011. Dectin-1 diversifies Aspergillus fumigatus-specific T cell responses by inhibiting $\mathrm{T}$ helper type $1 \mathrm{CD} 4 \mathrm{~T}$ cell differentiation. The Journal of Experimental Medicine 208: 369-381.

120. Grazziutti, M., D. Przepiorka, J. Rex, I. Braunschweig, S. Vadhan-Raj, and C. Savary. 2001. Dendritic cell-mediated stimulation of the in vitro lymphocyte response to Aspergillus. Bone Marrow Transplantation 27: 647-652.

121. Montagnoli, C., A. Bacci, S. Bozza, R. Gaziano, S. Fiorucci, A. Spreca, and L. Romani. 2001. The plasticity of dendritic cells at the host/fungal interface. Immunobiology 204: 582-589. 
122. Montagnoli, C., A. Bacci, S. Bozza, R. Gaziano, A. Spreca, and L. Romani. 2002. The interaction of fungi with dendritic cells: implications for Th immunity and vaccination. Current Molecular Medicine 2: 507-524.

123. Cenci, E., A. Mencacci, C. Fè d'Ostiani, G. Del Sero, P. Mosci, C. Montagnoli, A. Bacci, and L. Romani. 1998. Cytokine- and T Helper-Dependent Lung Mucosal Immunity in Mice with Invasive Pulmonary Aspergillosis. Journal of Infectious Diseases 178: 17501760 .

124. Hebart, H., C. Bollinger, P. Fisch, J. Sarfati, C. Meisner, M. Baur, J. Loeffler, M. Monod, J.-P. Latgé, and H. Einsele. 2002. Analysis of T-cell responses to Aspergillus fumigatus antigens in healthy individuals and patients with hematologic malignancies. Blood 100: 4521-4528.

125. Murdock, B. J., A. B. Shreiner, R. A. McDonald, J. J. Osterholzer, E. S. White, G. B. Toews, and G. B. Huffnagle. 2011. Coevolution of TH1, TH2, and TH17 responses during repeated pulmonary exposure to Aspergillus fumigatus conidia. Infect. Immun. 79: 125-135.

126. Korn, T., E. Bettell, M. Oukka, and V. K. Kuchroo. 2009. IL-17 and Th17 Cells. Annual Review of Immunology 27: 485-517.

127. Cenci, E., S. Perito, K. H. Enssle, P. Mosci, J. P. Latgé, L. Romani, and F. Bistoni. 1997. Th1 and Th2 cytokines in mice with invasive aspergillosis. Infection and Immunity 65: 564-570.

128. Stuehler, C., N. Khanna, S. Bozza, T. Zelante, S. Moretti, M. Kruhm, S. Lurati, B. Conrad, E. Worschech, S. Stevanović, S. Krappmann, H. Einsele, J.-P. Latgé, J. Loeffler, L. Romani, and M. S. Topp. 2011. Cross-protective TH1 immunity against Aspergillus fumigatus and Candida albicans. Blood 117: 5881-5891.

129. Porter, P. C., L. Roberts, A. Fields, M. Knight, Y. Qian, G. L. Delclos, S. Han, F. Kheradmand, and D. B. Corry. 2011. Necessary and sufficient role for $T$ helper cells to prevent fungal dissemination in allergic lung disease. Infection and Immunity 79: 44594471.

130. Tao, J., B. H. Segal, C. Eppolito, Q. Li, C. G. Dennis, R. Youn, and P. A. Shrikant. 2006. Aspergillus fumigatus extract differentially regulates antigen-specific CD4+ and CD8+ T cell responses to promote host immunity. Journal of Leukocyte Biology 80: 529-537.

131. Templeton, S. P., A. D. Buskirk, B. Law, B. J. Green, and D. H. Beezhold. 2011. Role of germination in murine airway CD8 T-cell responses to Aspergillus conidia. PLoS ONE 6: e18777.

132. Pahl, H. L., B. Krauss, K. Schulze-Osthoff, T. Decker, E. B. Traenckner, M. Vogt, C. Myers, T. Parks, P. Warring, A. Mühlbacher, A. P. Czernilofsky, and P. A. Baeuerle. 1996. The immunosuppressive fungal metabolite gliotoxin specifically inhibits transcription factor NF-kappaB. The Journal of Experimental Medicine 183: 1829-1840.

133. Patterson, R., M. Roberts, A. Ghory, and P. Greenberger. 1980. IgA antibody against Aspergillus fumigatus antigen in patients with allergic bronchopulmonary aspergillosis. Clin. exp. Immunol 42: 395-398.

134. Brakhage, A. A., K. Langfelder, G. Wanner, A. Schmidt, and B. Jahn. 1999. Pigment biosynthes is and virulence. Contributions to Microbiology 2: 205-215.

135. Jahn, B., F. Boukhallouk, J. Lotz, K. Langfelder, G. Wanner, and A. A. Brakhage. 2000. Interaction of human phagocytes with pigmentless Aspergillus conidia. Infection and Immunity 68: 3736-3739. 
136. Jahn, B., A. Koch, A. Schmidt, G. Wanner, H. Gehringer, S. Bhakdi, and A. A. Brakhage. 1997. Isolation and characterization of a pigmentless-conidium mutant of Aspergillus fumigatus with altered conidial surface and reduced virulence. Infection and Immunity 65: 5110-5117.

137. Langfelder, K., B. Jahn, H. Gehringer, A. Schmidt, G. Wanner, and A. A. Brakhage. 1998. Identification of a polyketide synthase gene (pksP) of Aspergillus fumigatus involved in conidial pigment biosynthesis and virulence. Medical Microbiology and Immunology 187: 79-89.

138. Tsai, H. F., R. G. Washburn, Y. C. Chang, and K. J. Kwon Chung. 1997. Aspergillus fumigatus arp1 modulates conidial pigmentation and complement deposition. Molecular microbiology 26: 175-183.

139. Tsai, H. F., M. H. Wheeler, Y. C. Chang, and K. J. Kwon-Chung. 1999. A developmentally regulated gene cluster involved in conidial pigment biosynthesis in Aspergillus fumigatus. Journal of Bacteriology 181: 6469-6477.

140. Volling, K., A. Thywissen, A. A. Brakhage, and H. P. Saluz. 2011. Phagocytosis of melanized Aspergillus conidia by macrophages exerts cytoprotective effects by sustained PI3K/Akt signalling. Cellular Microbiology 13: 1130-1148.

141. Thywiben, A., T. Heinekamp, H.-M. Dahse, J. Schmaler-Ripcke, S. Nietsche, P. F. Zipfel, and A. A. Brakhage. 2011. Conidial dihydroxynaphthalene melanin of the human pathogenic fungus Aspergillus fumigatus interferes with the host endocytosis pathway. Frontiers in Microbiology 2.

142. Tsai, H. F., Y. C. Chang, R. G. Washburn, M. H. Wheeler, and K. J. Kwon Chung. 1998. The developmentally regulated alb1 gene of Aspergillus fumigatus: its role in modulation of conidial morphology and virulence. Journal of bacteriology 180: 3031-3038.

143. Chai, L. Y. A., M. G. Netea, J. Sugui, A. G. Vonk, W. W. J. van de Sande, A. Warris, K. J. Kwon-Chung, and B. Jan Kullberg. 2010. Aspergillus fumigatus conidial melanin modulates host cytokine response. Immunobiology 215: 915-920.

144. Templeton, S. P., A. D. Buskirk, B. J. Green, D. H. Beezhold, and D. Schmechel. 2010. Murine models of airway fungal exposure and allergic sensitization. Medical Mycology 48: 217-228.

145. Mitchell, C. G., J. Slight, and K. Donaldson. 1997. Diffusible component from the spore surface of the fungus Aspergillus fumigatus which inhibits the macrophage oxidative burst is distinct from gliotoxin and other hyphal toxins. Thorax 52: 796-801.

146. Samarasinghe, A. E., S. A. Hoselton, and J. M. Schuh. 2011. A comparison between intratracheal and inhalation delivery of Aspergillus fumigatus conidia in the development of fungal allergic asthma in C57BL/6 mice. Fungal Biology 115: 21-29.

147. Jarvis, B. B., W. G. Sorenson, E.-L. Hintikka, M. Nikulin, Y. Zhou, J. Jiang, S. Wang, S. Hinkley, R. A. Etzel, and D. Dearborn. 1998. Study of toxin production by isolates of Stachybotrys chartarum and Memnoniella echinata isolated during a study of pulmonary hemosiderosis in infants. Applied and Environmental Microbiology 64: 3620-3625. 


\section{Chapter 2}

Pulmonary immune responses to Aspergillus fumigatus conidia in an immunocompetent mouse model of repeated aspiration exposures 


\section{INTRODUCTION}

Filamentous fungi are ubiquitous, saprophytic microorganisms that acquire nutrients from decaying plant matter and other carbon-rich substrates. Conidia formed by these fungi can be aerosolized following environmental disturbance. Certain conidia are sized within the respirable fraction and can be inhaled and deposited deep within the lungs (1). In small numbers, these conidia are rapidly phagocytosed and degraded by alveolar macrophages with little immunological consequence $(1,2)$. However, repeated exposures to higher concentrations may lead to the persistence of conidia within the lung and induction of airway inflammation, fungal allergy, and asthma.

Among the filamentous fungi, the opportunistic pathogen Aspergillus fumigatus is an etiological agent of invasive aspergillosis, hypersensitivity pneumonitis, allergy, and asthma (2). A. fumigatus-associated diseases have been steadily increasing due to improved therapeutic interventions for critically-ill patients resulting in increased numbers of immunosuppressed patients that are highly susceptible to fungal infections. Due to the extreme clinical impact of invasive disease, the majority of $A$. fumigatus exposure studies have examined the pathogenicity of the fungus in immunocompromised mice models. However, there has also been a steady increase in the incidence of allergy, including fungal allergies, in the immunocompetent population $(3,4)$. A. fumigatus-specific allergy and allergic bronchopulmonary aspergillosis have been reported in $23-80 \%$ and $12-40 \%$ of asthmatic patients, respectively (4-6). As a result, a larger population is affected by fungal-specific allergies than by invasive disease. Therefore, in order to better understand fungal diseases, it is necessary to determine the factors that direct the development of protective and/or allergic immune responses to A. fumigatus.

Previous reports have identified A. fumigatus-associated virulence factors including; thermotolerance, production of secondary metabolites (gliotoxin) and proteases, as well as cell wall-associated molecules such as $\alpha$ and $\beta$-glucans, galactomannans, and melanins (7). Melanins are large, polymeric pigments associated with the cell wall, and are highly resistant to acidic degradation, thereby contributing to the rigidity and integrity of the conidia. They are also responsible for the characteristic blue-green pigmentation observed in A. fumigatus wild-type (WT) conidia (8). Since fungi are primarily associated with external environments, melanin functions to protect the conidia from ultraviolet radiation and ensures the integrity of conidia under the stress of turgor pressure in plants (9-11). 
Using melanin knock-downs and albino mutants, melanins were found to enhance conidial survival by quenching reactive oxygen species (ROS) and preventing the binding of complement protein $\mathrm{C} 3$ to the surface of the conidia $(12,13)$. Melanin also protects conidia from the innate immune system by preventing phagolysosome acidification and inhibiting host-cell apoptosis (13-16). Conidia from melanin mutants exhibit decreased virulence in a mouse model of invasive aspergillosis $(16,17)$. The presence of melanin in A. fumigatus conidia was shown to attenuate the host pro-inflammatory cytokine response of human peripheral blood mononuclear cells, as albino mutant conidia induce higher levels of IL-6, TNF- $\alpha$, and IL-10 than wild-type conidia (18). Similar results were found with low melanin producing mutants of C. neoformans, as these conidia induce greater inflammatory responses and are cleared more rapidly, as well as induce greater $\mathrm{CD}^{+} \mathrm{T}$ cell responses and TNF- $\alpha$ levels (19).

Because fungal melanins protect conidia from innate clearance mechanisms, the persistence of conidia within the lung could alter the pulmonary immune responses to enhance the induction of allergy, asthma, and/or hypersensitivity pneumonitis. In an attempt to address a major caveat mentioned in Chapter 1, the role of phenotypic characteristics in the induction of allergy and/or asthma, this study was completed to examine how melanin may influence the induction of allergic fungal responses. Multiple exposures were used in this study to resemble repeated environmental exposures. Two strains of conidia with melanin synthesis pathway mutations derived from a clinical isolate of A. fumigatus were used. The Aarp2 mutant has a single gene deletion for the tetrahydroxynapthalene reductase and exhibits tan pigmentation, while the $\Delta a l b 1$ mutant has an albino appearance as a result of deleting the gene coding for the polyketide synthase in the dihydroxynapthalene (DHN) melanin synthesis pathway (20). These studies characterized the immune responses to melanin-deficient conidia in immunocompetent $\mathrm{BALB} / \mathrm{c}$ mice. For the first time, our results demonstrate a $\mathrm{CD}^{+} \mathrm{IL}-17^{+}$(Tc17) response and that conidia derived from melanin mutants resulted in increased eosinophilia and decreased neutrophils and $\mathrm{CD}^{+} \mathrm{IL}-17$ (Tc17) responses, as well as increased conidial clearance at early time points. 


\section{MATERIALS AND METHODS}

Growth and handling of fungi:

Fungal strains (Aspergillus fumigatus B-5233 (ATCC 13073)- the wild-type (WT) parent strain, $\triangle a r p 2$, and $\triangle a l b 1)$ were received as a gift from Dr. June Kwon-Chung (NIAID, Bethesda, MD) (20). Fungi were grown for 14 days on malt extract agar (MEA) plates at $25^{\circ} \mathrm{C}$ in low humidity conditions to minimize the production of hyphae. Fungal conidia were harvested from plates by applying $1 \mathrm{~g}$ of $0.5 \mathrm{~mm}$ glass beads (BioSpec Products Inc., Bartlesville, OK) and gently shaking. The bead/conidia mixture was collected in a tube and suspended in $1 \mathrm{ml}$ sterile phosphate buffered saline, pH 7.4 (PBS). The beads were vortexed and the supernatant containing conidia was collected and enumerated using a hemocytometer. To avoid the loss of fungal antigens, the conidia were subsequently diluted in sterile PBS, without washing, to a final concentration of $4 \times 10^{7}$ conidia/ml $\left(2 \times 10^{6}\right.$ conidia/50 $\mu$ l) for animal exposures, as previously reported (21). Fresh conidial suspensions were prepared from 14 day old cultures for each exposure.

\section{MALDI-qTOF MS analysis of melanin mutant conidia:}

For positive ion matrix-assisted laser desorption/ionization quadrupole time-of-flight mass spectrometry (+MALDI qTOF MS) analysis, conidia were harvested as previously described (22). Briefly, conidia $\left(\sim 1 \times 10^{8}\right)$ isolated from three plates of each A. fumigatus strain were mixed with $100 \mu \mathrm{l}$ of $0.1 \mathrm{~mm}$ zirconium beads (BioSpec, Bartlesville, OK) and $1 \mathrm{ml}$ of $50 / 50$ acetonitrile $/ 4 \%$ trifluoroacetic acid (TFA) in water. After three one-minute cycles of bead beating, the samples were centrifuged at $14,500 \mathrm{rpm}$ for 10 minutes. The supernatant was mixed 1:1 with $10 \mathrm{mg} / \mathrm{ml} \alpha$-cyano-4-hydroxycinnamic acid (50/50 acetonitrile/0.1\% TFA) and $1 \mu 1$ spotted on the target plate and allowed to air dry. MALDI-qTOF mass spectra were acquired using a MALDI-SYNAPT MS (Waters Corporation, Milford, MA) qTOF mass spectrometer capable of mass resolution $(\mathrm{m} / \mathrm{Dm})$ of 14,000 and mass accuracy of $\pm 5 \mathrm{ppm}$. Spectra were acquired over the $\mathrm{m} / \mathrm{z}$ range of 3000 to $14000 \mathrm{u}$. Composite mass spectra were the result of a 6.5 minute acquisition with the frequency-tripled Nd:YAG laser $(355 \mathrm{~nm})$ operating at $200 \mathrm{~Hz}$, with the laser pulse energy maintained just above the threshold for ion production. Mass spectra were acquired using a predetermined "spiral" pattern that was held constant for all sample deposits, ensuring that a reproducible surface area was irradiated for each sample. 


\section{Polyacrylamide Gel Electrophoresis:}

Conidial extracts were prepared by adding $2 \mathrm{ml}$ of phosphate buffered saline $/ 0.1 \%$ Tween to each of four plates and the conidia were agitated from the surface using a sterile inoculating loop. The conidial suspension was centrifuged at $2000 \mathrm{rpm}$ for five minutes. The supernatant was discarded and the pellet containing the conidia was resuspended in sodium bicarbonate buffer (pH 8.0) and rocked at $4^{\circ} \mathrm{C}$ overnight. The sample was then centrifuged and the pellet was frozen at $-80^{\circ} \mathrm{C}$ overnight, and lyophilized. Following lyophilization, the sample was mixed with 0.1 $\mathrm{mm}$ glass beads and bead beat for three one-minute cycles using a mini bead-beater (BioSpec, Bartlesville, OK). Sodium bicarbonate buffer $(2 \mathrm{ml})$ was added and the samples were again subjected to three one-minute bead-beating cycles, centrifuged, and the resulting supernatant was used for SDS-PAGE. Protein concentrations were determined using a $\mathrm{BCA}^{\mathrm{TM}}$ protein assay kit as per manufacturer's instructions (Thermo Scientific, Waltham, MA). A $12 \%$ separating gel with a $4 \%$ stacking gel was used for SDS-PAGE analysis. Conidial extracts $(30 \mu \mathrm{g})$ were mixed with Laemmli's sample buffer and heated at $95^{\circ} \mathrm{C}$ for 5 minutes. Samples were separated by electrophoresis for 90 minutes at $100 \mathrm{~V}$. The separating gel was then stained using the Imperial Blue stain according to manufacturer's instructions (Thermo Scientific, Waltham, MA).

Field emission scanning electron microscopy:

A $1 \times 1 \mathrm{~cm}^{2}$ sample of agar was isolated from 14 day old culture plates of each $A$. fumigatus strain. The sample was air dried for three days, attached to an aluminum mount with double-stick carbon tape, and sputter coated with gold/palladium. Images were collected on a Hitachi (Tokyo, Japan) S-4800 field emission scanning electron microscope.

\section{Animals:}

Female BALB/c mice, aged 5-7 weeks (Jackson Laboratory, Bar Harbor, ME), were acclimated for approximately one week before initial exposures. The mice were housed in filtered, ventilated polycarbonate cages in groups of five on autoclaved hardwood chip bedding. The temperature in the animal facility was maintained between $68^{\circ} \mathrm{F}$ and $72^{\circ} \mathrm{F}$ and the relative humidity between $36 \%$ and $57 \%$. The light/dark cycle was maintained on 12-hour intervals. Mice were provided NIH-31 modified 6\% irradiated rodent diet (Harlan, Teklad) and tap water 
ad libitum. Sentinel mice were free of viral pathogens, parasites, mycoplasmas, Helicobacter, and cilia-associated respiratory (CAR) Bacillus. The National Institute for Occupational Safety and Health (NIOSH) animal facility is an environmentally controlled barrier facility that is fully accredited by the Association for the Assessment and Accreditations of Laboratory Animal Care International. All animal procedures were performed under a NIOSH Animal Care and Use Committee approved protocol (protocol \# 08-ST-M-015).

\section{Animal exposures:}

Mice were exposed to fungal suspensions by involuntary aspiration as previously described (23). Briefly, mice were anesthetized using isoflurane (Webster Veterinary Supply Inc., Devens, MA), suspended on a slant board, the tongue was held in full extension and a $50 \mu 1$ suspension of $2 \times 10^{6}$ conidia placed at the base of the tongue. The tongue was restrained for two full breaths while the mice inhaled the conidial suspension. The anesthetized mice were then returned to the cage and allowed to recover.

To assess responses to repeated exposures used to resemble repeated natural environmental exposures, mice aspirated 14 day old conidia twice per week for two weeks, rested for two weeks, and then challenged a final time (Figure 2.3A). Three days post-challenge, mice were sacrificed via an intraperitoneal (i.p.) injection of sodium pentobarbital (Sleepaway®), Fort Dodge Animal Health, Fort Dodge, IA).

To examine the impact of innate immunity compared to adaptive immunity on the clearance of conidia, mice were either repeatedly exposed to conidia as indicated in Figure 2.3A (adaptive response), or mock-exposed with sterile saline twice per week for two weeks, rested for two weeks, and then exposed a final time to the indicated conidia (innate response). Mice were sacrificed at the indicated time points and lungs were removed and homogenized in sterile saline. Homogenates were plated in triplicate and incubated at $25^{\circ} \mathrm{C}$ for 24 hours. At this time, colony counts were visually determined.

\section{Histology:}

A group of five mice per fungal strain were repeatedly exposed as indicated in Figure 2.3A. These mice were then sacrificed 72 hours post final exposure. The descending aorta and the inferior vena cava were severed, and then the lungs were perfused by injecting $5 \mathrm{ml}$ of PBS 
followed by $5 \mathrm{ml}$ of $10 \%$ formalin buffered saline (FBS, Fisher Scientific, Fairlawn, NJ) into the right ventricle. No bronchoalveolar lavage fluid (BALF) was taken from these animals. The trachea was then exposed, nicked, and a catheter was inserted into the trachea. A syringe containing $1 \mathrm{ml}$ of $10 \%$ FBS was then injected into the lungs, and the trachea was tied closed. The lungs were then removed and fixed in 10\% FBS for 3-5 days prior to histological processing. Tissue processing, embedding, hematoxylin and eosin (H\&E) and Grocott's Methenamine Silver (GMS) staining were performed by West Virginia University Tissue Bank (Morgantown, WV).

To assess the frequency of germinated conidia in lung tissues of mice exposed to fungal conidia, 150 fields of view from GMS stained mid-coronal sections of the lungs of each animal were examined for conidia, swollen conidia, and germ tube formation. Swollen conidia were defined by conidial swelling (2-3x normal size) as indicated in Appendix A3.

\section{Collection of BALF:}

For collection of BALF, the lungs were first perfused with $10 \mathrm{ml}$ of PBS as indicated above, and then the trachea was exposed. Following nicking the trachea, a catheter was inserted and tied off with a suture to prevent leakage. A syringe containing $1 \mathrm{ml}$ of PBS was attached to the catheter, the liquid injected into the lungs and subsequently removed. This process was repeated until $3 \mathrm{ml}$ of BALF was collected.

Flow Cytometric Analysis of BALF:

Differential cell staining

All reagents were obtained from BD Biosciences (BD Biosciences, San Jose, CA) unless otherwise specified. BALF cell composition was determined by flow cytometric analysis of recovered lavage cells in suspension. BALF was centrifuged for five minutes at $1500 \mathrm{rpm}$, the supernatant discarded, and the cell pellet resuspended and washed in $1 \mathrm{ml}$ of FACS buffer (PBS, $5 \%$ fetal bovine serum, and $0.05 \%$ sodium azide). The washed pellet was resuspended, and divided in half for cell enumeration and intracellular cytokine staining. One half of the cells were stained in FACS buffer, 10\% rat serum, Fc-receptor blocking antibody (clone 24G2) and the following antibodies: rat anti-mouse Ly-6G FITC, rat anti-mouse Siglec-F PE, pan-leukocyte rat anti-mouse CD45 PerCP, and rat anti-mouse CD11c APC. After staining 30 minutes on ice in the 
dark, cells were washed with FACS buffer and fixed with BD Cytofix for 10 minutes. Cells were then washed and resuspended in FACS buffer for flow cytometry. Populations of cells were evaluated by flow cytometric analysis on a BD LSRII (BD Biosciences, San Jose, CA). Neutrophils were defined as $\mathrm{CD} 45^{\mathrm{hi}} \mathrm{Ly}-6 \mathrm{G}^{\text {hi }} \mathrm{CD} 11 \mathrm{c}^{\text {low }}$, eosinophils as Ly$6 \mathrm{G}^{\text {low }}$ Siglec $\mathrm{F}^{\text {hi }} \mathrm{CD} 11 \mathrm{c}^{\text {low }}$, and alveolar macrophages as $\mathrm{Ly}-6 \mathrm{G}^{\text {low }}$ Siglec $\mathrm{F}^{\text {hi }} \mathrm{CD} 11 \mathrm{c}$ hi , as previously reported (24). Total cell numbers were quantified from the BALF via hemocytometer counts. Total numbers of each cell population were obtained by multiplying the frequency of specific population by the total number of BALF cells recovered for each animal.

\section{Intracellular cytokine staining}

In a separate tube, BALF T-cells were quantified using rat anti-mouse CD8 FITC and CD4 PerCP antibodies. T-cell cytokine production was determined by fluorescent intracellular cytokine staining (ICS) as previously described (25). Briefly, the BALF suspension was centrifuged for five minutes at $1500 \mathrm{rpm}$ and washed in $1 \mathrm{ml}$ of RPMI 1640 complete medium (GIBCO, Grand Island, NY). The supernatant was discarded and a solution of Leukocyte Activation Cocktail with GolgiPlug in $0.2 \mathrm{ml}$ complete medium was added to each sample for stimulation of cytokine production and simultaneous inhibition of cytokine secretion. Cells were incubated at $37^{\circ} \mathrm{C}$ in $5 \% \mathrm{CO}_{2}$ for four hours. After incubation, the cells were washed in FACS buffer and stained for flow cytometry using rat anti-mouse CD4 PerCP and rat anti-mouse CD8 FITC on ice in the dark for 30 minutes. Cells were then washed in FACS buffer and centrifuged, and the cell pellets were resuspended in BD Cytofix/Cytoperm for 10 minutes to allow for fixation and permeabilization required for subsequent ICS. Cells were washed with $1 \mathrm{ml}$ of BD PermWash, and resuspended in PermWash. Each sample was divided equally into two tubes and stained with rat anti-mouse IFN- $\gamma$ APC, rat anti-mouse TNF $\alpha$ PE-Cy7, and rat anti-mouse IL-17 $\mathrm{PE}$, or with control isotype antibodies (eBioscience, San Diego, CA). Cell were collected on a BD LSRII for one minute or until 50,000 events were recorded, with lymphocytes gated on the basis of low forward and side scatter, then subsequently gated on $\mathrm{CD}^{+}$or $\mathrm{CD} 8^{+}$populations to determine intracellular expression of cytokines. 


\section{Data Analysis Methods:}

Analysis of flow cytometric samples was performed with FlowJo software (TreeStar, Ashland, OR). GraphPad Prism was used for generation of graphs and statistical analysis for clearance and conidial germination experiments (GraphPad Software, La Jolla, CA). The data were analyzed via two way analysis of variance (26) followed by Bonferroni post-test. Statistical analysis of intracellular cytokine producing cells was performed using SAS version 9.2 for Windows (SAS, Cary, NC). The data were log transformed prior to analysis and ProcMix was used to run a one way analysis of variance (26) with 'experiment' included as a random factor to measure statistical significance. Differences between experimental groups that resulted in a $p$ value of less than 0.05 were considered significant.

\section{RESULTS}

MALDI mass spectra of melanin-deficient conidia are the same as wild-type A. fumigatus

Although the genetic alterations in the melanin pathways of the strains used in this study have been previously characterized, it is unknown if the gene deletions in the melanin mutant conidia impacted other proteins that could potentially alter the immune responses to these conidia. Therefore, the MALDI 'fingerprint' mass spectra of extracts from conidia of each strain were examined. We have previously demonstrated the utility of mass spectrometry to 'fingerprint' fungi for species and strain-specific discrimination $(22,27,28)$. Figure 2.1 A-C shows the +MALDI qTOF MS fingerprint mass spectra for the different A. fumigatus strains. The mass spectra show the presence of multiple peptide/protein peaks, with prominent peaks at 4840, 7875, and 8575 u. These mass spectra are in good agreement with the A. fumigatus spectral fingerprint previously reported (22). The peak height of protein/peptide signals in the $\Delta a l b 1$ mutant strain spectrum were greater than those of the WT and $\Delta$ arp2 spectra (Supplemental Appendix A1); consistent with previous observations that fungal-derived pigments suppress the desorption and/or ionization process during MALDI-TOF MS analysis (29).

In addition to high resolution +MALDI qTOF MS analysis of the $<15 \mathrm{kDa}$ mass range, SDS PAGE was additionally performed to identify potential differences that may occur between high molecular weight proteins of the different strains. As illustrated in Figure $2.1 \mathrm{D}$, the protein patterns of equally loaded melanin-deficient mutant conidial extracts is similar to that of WT $A$. 
fumigatus. The protein profile for each of the strains, however, does contain observable differences in the density of some bands that are less than $30 \mathrm{kDa}$ size range. These results suggest that global protein synthesis was not significantly altered by the gene deletions in the melanin-deficient conidia. 
Figure 2.1. +MALDI qTOF MS fingerprint mass spectra

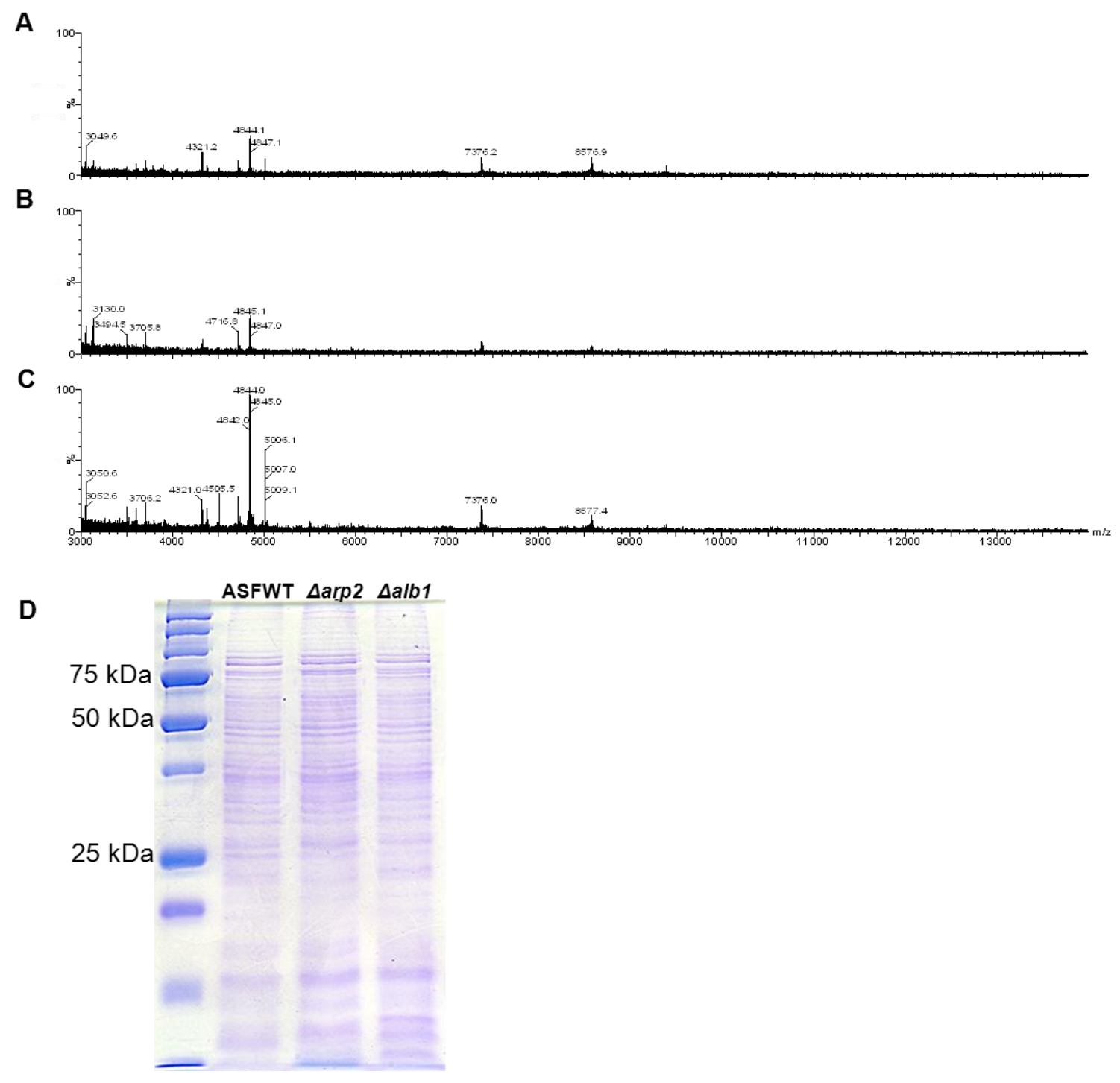

Figure 2.1. +MALDI qTOF MS fingerprint mass spectra. A) A. fumigatus WT B) Sarp2 and C) $\triangle a l b 1$. Spectra are representative of three independent fungal cultures. Spectra are presented on fixed $y$-axis (\% relative abundance) and optimized between the $\mathrm{m} / \mathrm{z}$ range of $3000-14000 u$. D) SDS page banding pattern of conidial extracts. 


\section{Melanin-deficient conidia retain the Rodlet A layer}

The interaction of fungi with immune cells occurs through the interaction of pattern recognition receptors (PRRs) on host immune cells and the pathogen-associated molecular patterns (PAMPs) on fungal conidia and hyphae. Non-germinated A. fumigatus conidia contain a hydrophobic protein layer termed the Rodlet A (RodA) layer. This layer is immunologically inert and has been shown to protect the conidia from innate immune recognition by masking the PAMPs and preventing recognition by the innate immune system (30). Therefore, it was necessary to determine if the melanin-deficient conidia retained the RodA layer to ensure that any differences in the immune responses were not due to the differential accessibility of immunostimulatory cell wall components masked by the RodA layer. Field emission scanning electron microscopy (Figure $2.2 \mathrm{~A}-\mathrm{C}$ ) showed the surface of the albino ( $\triangle a l b 1)$ conidia appeared smoother than that of WT or $\triangle a r p 2$; however, this melanin mutant strain retained a RodA layer similar to that observed in WT conidia. Taken together with the mass spectrometry data and SDS-PAGE analysis, these data suggest that these strains differ primarily in melanin content.

Figure 2.2 Field emission scanning electron microscopy images.

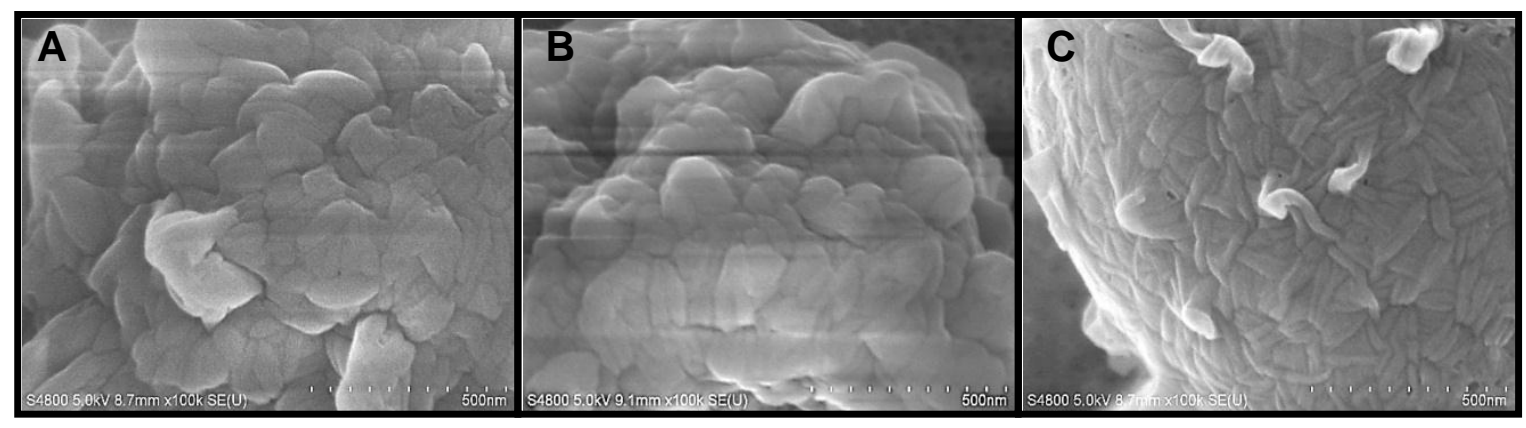

Figure 2.2. Field emission scanning electron microscopy images. A) WT, B) $\triangle a r p 2$, and C) $\Delta a l b 1$ conidia showing the presence of the RodA layer. 
Repeated exposures to A. fumigatus DHN-melanin-deficient conidia results in similar pathological inflammation compared to wild-type conidia.

Next we sought to determine if there was a difference in the inflammatory response following exposures to WT, $\triangle a r p 2$, or $\triangle a l b 1$ mutants using immunocompetent BALB/c mice. Mice were repeatedly exposed to conidia via pharyngeal aspiration as indicated in Figure 2.3A. This exposure method was initially used due to the large quantity of historic data showing high reproducibility and efficiency for the pulmonary delivery of fungal conidia. Moderate to severe inflammation and airway remodeling, resembling hypersensitivity pneumonitis, were evident in all mice regardless of the A. fumigatus strain (Figure $2.3 \mathrm{~B}-\mathrm{E}$ ). The extensive granuloma formation, mucus production, bronchoalveolar lymphoid tissue (BALT) induction, and goblet cell hyperplasia were histologically similar between the three exposure groups.

Melanin-deficient conidial exposures result in different polymorphonuclear leukocyte responses.

Airway cellularity following exposures was examined in BALF by flow cytometry. Total cell numbers were comparable between exposure groups; however, $\triangle a l b 1$ conidia exposures induced fewer numbers of neutrophils (Figure 2.3 F-G). There was a concomitant increase in eosinophils in this group of animals (Figure $2.3 \mathrm{H}$ ). Interestingly, eosinophils were also significantly increased in mice exposed to $\triangle a r p 2$ conidia when compared to WT exposed mice (Figure $2.3 \mathrm{H}$ ). However, this increase in eosinophils did not correlate to the production of a serum antibody response, as no increase in total $\operatorname{IgE}$ or fungal-specific $\operatorname{IgG}$ antibodies could be detected in any of the exposed animals (data not shown). 
Figure 2.3 Exposure schedule and characterization of lung inflammation

A

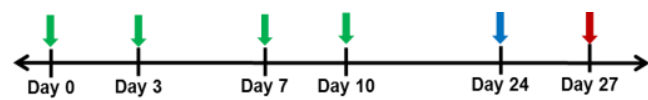

$\downarrow=$ Exposure

$\downarrow=$ Challenge

$\downarrow$ = Sacrifice $72 \mathrm{hrs}$ post-challenge

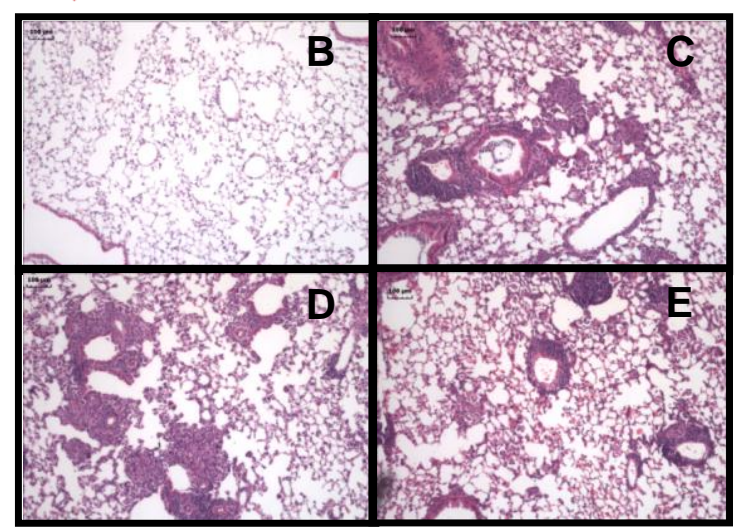

G

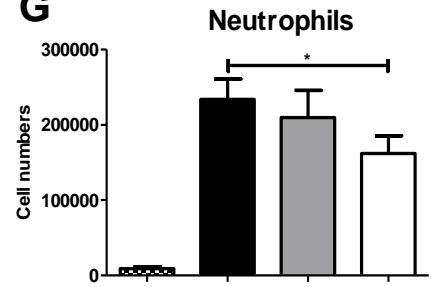

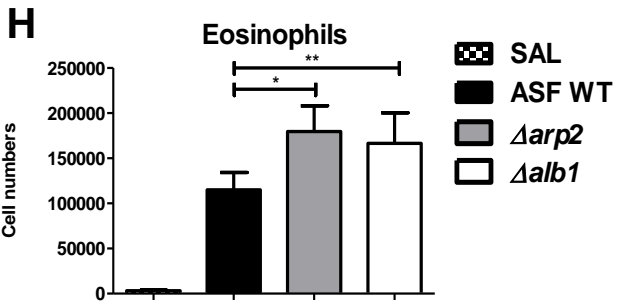

Figure 2.3. Exposure schedule and characterization of lung inflammation in repeatedly exposed mice. A) Exposure schedule and representative $H \& E$ stained lung sections from exposures to $B$ ) saline only, C) WT conidia, D) Aarp2 conidia, and E) Aalb1 conidia. Graphs indicate total polymorphonuclear cells in BAL from exposed mice. F) Total cell numbers, G) neutrophils, and $H$ ) eosinophils. Data are presented as the average \pm standard error of measure of four independent experiments. $N=20$ mice/group. $S A L=$ saline only exposures. Statistical differences indicated by asterisks $* p \leq 0.02$ and $* * p \leq 0.05$ as determined by one-way ANOVA. 
CD8 $+I L-17+T$ cells are elevated in the lungs of mice exposed to $\triangle a l b 1$ DHN-melanin-deficient conidia.

To determine if there were differences in T-cell mediated responses to melanin-mutant strains, BALF was analyzed for $\mathrm{T}$ cells and intracellular cytokine production using flow cytometry. There was a decreasing trend in $\mathrm{CD}^{+} \mathrm{T}$ cell numbers that correlated with a decrease in melanin production. However, there was no significant difference in $\mathrm{CD}^{+}$or $\mathrm{CD} 8^{+} \mathrm{T}$ cell numbers (Figure $2.4 \mathrm{~A}$ and E). Additionally, there was no significant difference in $\mathrm{CD}^{+} \mathrm{T}$ cell cytokine production of IFN- $\gamma$ and IL-17 (Figure $2.4 \mathrm{~B}$ and C). An increase was observed in CD4 ${ }^{+}$ TNF $\alpha$ production, though this result was not statistically significant (Figure $2.4 \mathrm{D}$ ).

$\mathrm{CD}^{+} \mathrm{T}$ cell IFN $\gamma$ production was slightly elevated, but was not statistically significant, in mice exposed to $\Delta a l b 1$ conidia (Figure 2.4F). Interestingly, there was a significant decrease in the Aalb1 induced Tc17 cell population when compared to both WT and Aarp2 exposure groups (Figure 2.4G). CD8 ${ }^{+} \mathrm{TNF} \alpha$ production was consistent between the exposure groups (Figure $2.4 \mathrm{H})$. 
Figure 2.4 T cell cytokine production following multiple aspirations.

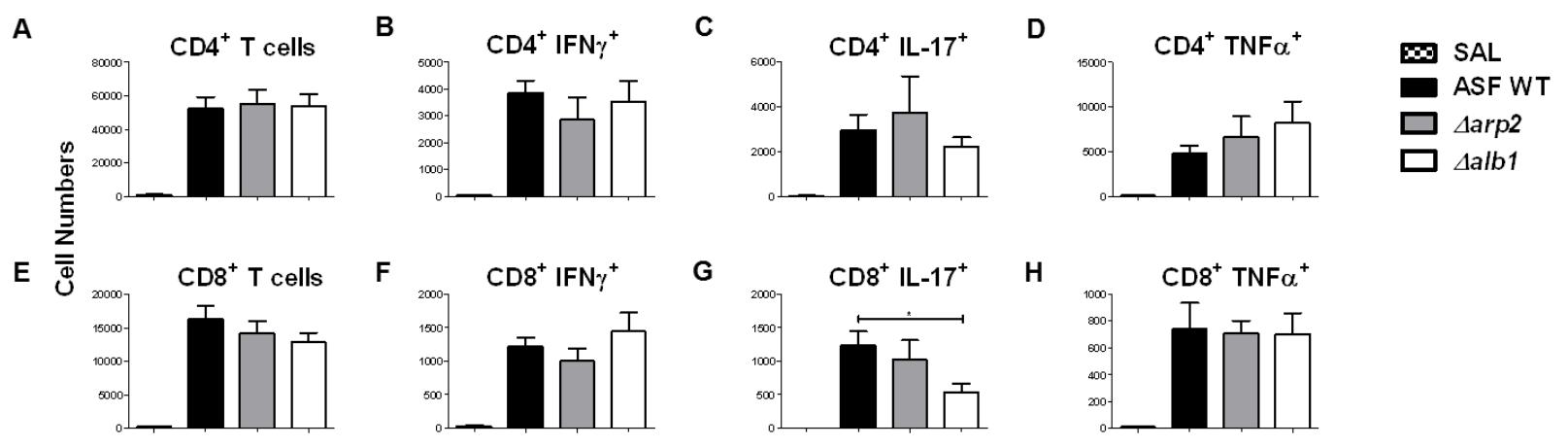

Figure 2.4. $T$ cell cytokine production following multiple aspirations. $C D 4+$ and $C D 8+T$ cell cytokine production in the BAL of mice exposed to WT, $\triangle a r p 2$, or $\triangle a l b 1$ conidia. CD4+ and $C D 8+T$ cells, CD4+ and CD8+IFN- $\gamma+$. Data are presented as the average \pm standard error of measure of four independent experiments. $N=20 . C D 4+$ and $C D 8+I L-17+$ and $T N F-\alpha+, N=10$

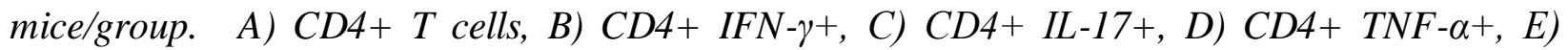
CD8+ T cells, F) CD8+IFN- - +, G) CD8+IL-17+, and H) CD8+ TNF- $\alpha+$. Statistical differences are indicated by asterisks $* p \leq 0.01$ as determined by one-way ANOVA.

Melanin-deficient conidia are cleared more rapidly from the airways of both sensitized and nonsensitized mice.

Histological examination of samples from repeatedly aspirated mice demonstrated that a larger number of WT conidia remained in the lungs of mice at the time of sacrifice than in mice exposed to the melanin mutant conidia (Figure 2.5 B-D and Supplementary Table 1-Appendix Table A3). Additionally, there were greater numbers of swollen conidia in the WT (7\% swollen conidia) and $\Delta a r p 2(3.8 \%)$ exposed mice than in $\Delta a l b 1$ (1.5\%) exposed mice. A higher frequency of germ tube formation was also observed in mice exposed to WT and $\Delta a r p 2$ conidia. No germ tubes were identified in any of the mice exposed to $\Delta a l b 1$ conidia (Figure 2.5E). These results were not due to differential viability, as each fungal strain exhibited similar viability prior to aspiration (data not shown). 
Figure 2.5 Conidial germination in the lung tissue of mice

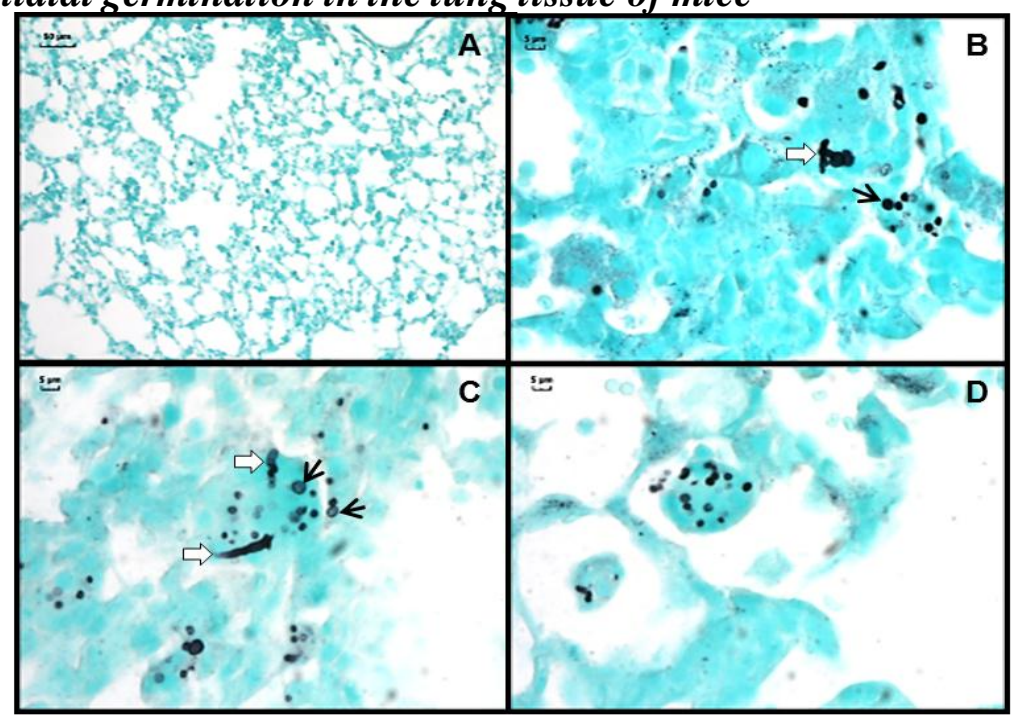

E

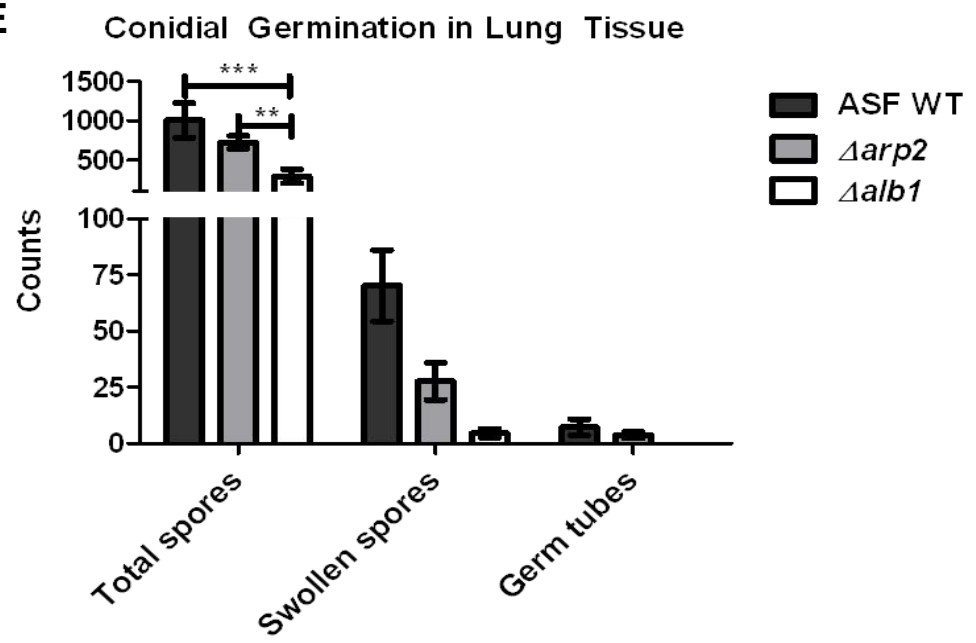

Figure 2.5. Conidial germination in the lung tissue of mice repeatedly exposed as indicated in Figure 2.3A. GMS stained lung section following exposures to A) saline only, B) WT conidia, C) Aarp2 conidia, and D) $\Delta$ alb1 conidia. Black arrows indicate swollen conidia (2-3X the size of resting conidia). White arrows indicate the formation of germ tubes. E) Quantification of total conidia, swollen conidia, or germ tube formation in WT, $\Delta$ arp2, and $\Delta$ alb1 exposed mice. $N=4$ mice for WT and $\Delta a l b 1, N=5$ mice for Aarp2. Statistical significance is indicated by asterisks, *** $p<0.0001$ and ** $p<0.01$, as determined by two-way ANOVA followed by Bonferroni post-test. 
To determine the lung clearance kinetics of each strain, we compared mice that were aspirated a single time (innate response- 4 exposures to saline only and challenged with conidia) to mice repeatedly aspirated (adaptive response- exposed as indicated in Figure 2.3A). The number of WT conidia remaining in the lung was significantly greater than $\triangle a r p 2$ and $\triangle a l b 1$ conidia at 5 hours post final exposure in both single and repeated exposure mice (Figure 2.6). By 24 hours post final exposure, greater than $94 \%$ of conidia were cleared in both single and multiple exposure mice despite the presence or absence of melanin (Figure 2.6). By 72 hours, more than $99 \%$ of the fungal conidia were removed from all mice, irrespective of melanin content. Interestingly, there were greater numbers of conidia remaining in the lungs of mice that repeatedly aspirated conidia at the 24 and 72 hour time points when compared to single exposure mice. This result was not dependent on the presence of melanin in the fungal conidia. 
Figure 2.6 Rate of conidial clearance in exposed mice.

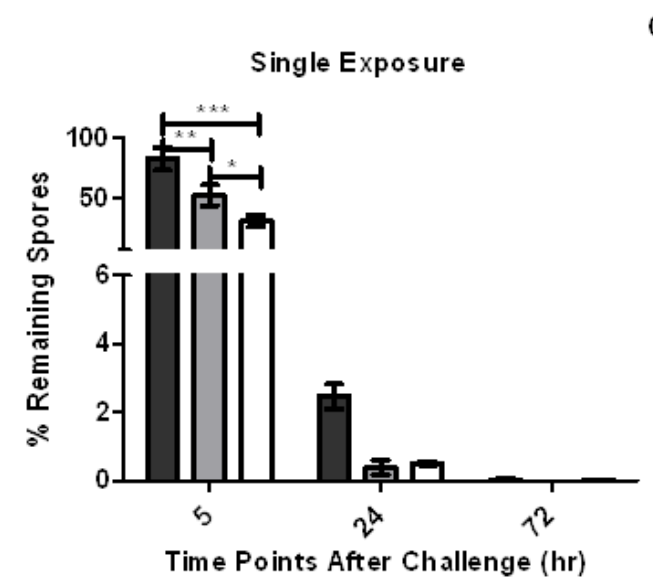

Clearance

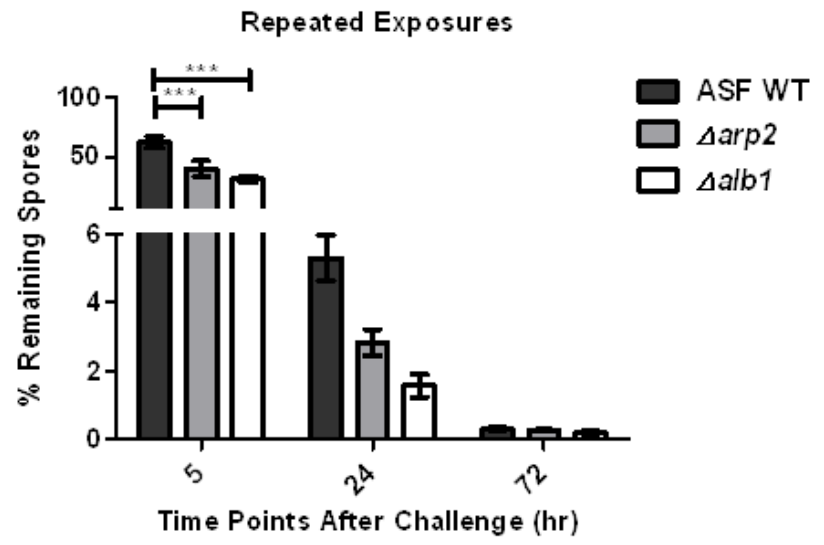

Figure 2.6. Rate of conidial clearance in exposed mice. Mice were exposed to WT, Larp2, or Aalb1 conidia and sacrificed at the indicated time points. At this time, lungs were removed, homogenized, and plated on MEA to determine fungal CFUs. Left panel shows clearance following a single conidial exposure in mice mock-exposed to saline 4 times and then challenged with the indicated fungal strain. Right panel shows adaptive clearance in mice repeatedly exposed to conidia as indicated in Figure 3A. Data are presented as the average \pm standard error of measure of two independent experiments. $N=10$ mice/group. Data from each strain were normalized to the total amount of conidia obtained from mice sacrificed immediately after exposure. $N=5$ mice/group. Statistical significance is indicated by asterisks, *** $p<0.0001$, ** $p<0.001$, and $* p<0.05$, as determined by two-way ANOVA followed by Bonferroni post-test. 


\section{DISCUSSION}

Although A. fumigatus is known to cause human disease, the fungal-specific factors that contribute to pulmonary immune responses, protection from infection, and allergy induction in immunocompetent hosts have not been completely elucidated. Fungi produce proteases that are common allergens, however, the role of other components that contribute to the development of fungal allergies have largely been ignored $(2,31,32)$. Fungal melanin has been shown to be an important virulence factor in invasive aspergillosis models, yet there is no information on the role of melanin on the development of allergic responses in an immunocompetent animal model $(16,17)$. The present study aimed to determine the impact of A. fumigatus conidial melanization on the pulmonary immune response in immunocompetent Balb/c mouse model. Multiple aspiration exposures were used to resemble continuous human exposures which can lead to allergy, asthma, or hypersensitivity responses (1). Though the central aim of this dissertation is to develop a murine model of repeated exposures to dry A. fumigatus conidia, the abundance of available information obtained from aspiration exposures made it an acceptable method for initial exposure studies. This also allowed for comparisons between dry conidia vs. conidial suspension exposures, and the ability to discern any potential differences between the two methods.

Previous studies have shown that the conidia from melanin mutant strains differ from WT conidia only in their melanin content and the smoothness of the outer cell wall surface (33). Our FESEM results confirmed that $\triangle a l b 1$ conidia have a smoother appearance (Figure-2.2). While there were observable differences in density of some SDS-page protein bands less than $30 \mathrm{kDa}$, there were no major differences in the protein profile of melanin mutant conidia compared to WT. Thus, the single gene deletions in the mutant strains did not appear to have an impact on global protein synthesis and it is unlikely that differential expression of potentially immunereactive proteins was solely responsible for the observed differences in the immune responses between the three strains.

A. fumigatus conidial walls are surrounded by an immunologically inert hydrophobic rodlet layer composed of RodA and RodB proteins with melanin polymers extensively intercalated throughout the wall (34). These layers help provide the conidia its structural rigidity. Importantly, the rodlet layer has been shown to protect the conidia from innate immune 
recognition by PRRs. Previous reports have shown that in vitro exposure of primary dendritic cells and macrophages to the RodA protein did not induce cellular maturation/activation. RodA did not stimulate the production of inflammatory cytokines, antibody, or protect from infection in an invasive aspergillosis animal model. However, $\triangle R o d A$ mutant conidia or swollen WT conidia were highly inflammatory and capable of activating innate immune cells (30).

Previous studies have shown contradicting results concerning the presence of RodA layer in melanin-deficient A. fumigatus conidia $(8,15,33)$. Pihet et al. used atomic force microscopy to examine the conidial surfaces of naturally-isolated A. fumigatus melanin mutant conidia and reported the absence of the RodA layer in these strains (8). However, it is possible that other mutations independent of the melanin pathway may have affected the rodlet layer in this naturally occurring mutant. Brakhage et al. examined the surface of laboratory-derived albino conidia, similar to the strain in the present study, using scanning electron microscopy and compared the conidial surfaces of WT and melanin-deficient conidia. In their studies the RodA layer remained intact in albino conidia $(15,33)$. Our results were similar, in that the rodlet layer was observed on the surface of the WT and each melanin mutant strain, with the rodlet layer of the albino mutants appearing in highly organized tight bundles. This finding is important as it suggests that the differences in the immune responses observed are potentially related to the difference in amount of melanin rather than any differences in accessibility of immunostimulatory cell wall components in dormant conidia, such as galactomannan or $\beta$ glucan. It has been suggested that immunostimulatory PAMPs, that may impact early innate recognition and clearance, are more easily accessible on the surface of melanin-deficient conidia [23]. It is also possible that because of the lack of melanin, the albino or melanin knock-down strains over-compensate by increasing the concentration of other immune-modulatory conidial wall components. However, further studies are required to determine if these cell wall components remain hidden by the rodlet layer in melanin-deficient conidia (18).

In this study, we found that histopathological inflammation, granuloma formation, BALT induction, goblet cell hyperplasia, and airway remodeling were similar in each exposure group. Therefore, the presence of melanin does not appear to significantly impact lung inflammation. However, the melanin mutant conidia stimulated greater numbers of eosinophils in the airways indicating a shift in the type of immune response. Unfortunately, a serum antibody response was not detectable, specific IgG or total $\operatorname{IgE}$ (data not shown), thus a clear $\mathrm{T}_{\mathrm{H}} 1$ to $\mathrm{T}_{\mathrm{H}} 2$ 
shift was not apparent. It is probable, that because all conidia retained the rodlet layer which is resistant to proteolytic degradation, the production of antigens for processing and presentation was inhibited (30). Additional experiments to determine the extent that melanization impacts antigen presentation to $\mathrm{B}$ cells, and therefore antibody production, will be crucial to determine the mechanisms of pulmonary tolerance in response to fungi.

It has been previously shown that a $\mathrm{T}_{\mathrm{H}} 1$ response, consisting of $\mathrm{CD}^{+} \mathrm{T}$ cells and IFN- $\gamma$, is necessary for the efficient clearance of fungal conidia $(2,35)$. Therefore, we evaluated the production of cytokine responses in $\mathrm{T}$ cells to determine if a similar $\mathrm{T}$ cell response occurs in the repeated exposure model. While $\mathrm{TNF} \alpha$ and IFN- $\gamma$ are known proinflammatory cytokines required for protective immunity against fungi, the role of IL-17 in fungal immunology is relatively unknown (36). IL-17 is associated with chronic inflammation, autoimmune disorders, and allergy, and involved in the recruitment and subsequent activation of neutrophils and macrophages to the site of inflammation $(37,38)$. The levels of CD4 ${ }^{+} \mathrm{IFN}-\gamma^{+}, \mathrm{TNF} \alpha^{+}$, and IL$17^{+}$cells were increased by fungal exposures, yet comparable between WT, Aarp2, and $\Delta a l b 1$ exposed mice. This indicates that the extent of melanization does not appear to affect these cells directly.

Considerably less is known about the role of $\mathrm{CD}^{+} \mathrm{T}$ cells in immune responses to fungi. $\mathrm{CD}^{+} \mathrm{T}$ cell responses were shown to be partly dependent on germination, and play a role in fungal clearance and protection from infection, although their mechanism of protection remains uncharacterized $(21,39)$. In the present study, we did not see changes in $\mathrm{CD} 8^{+} \mathrm{IFN}-\gamma^{+}$cell recruitment, however, $\mathrm{CD} 8^{+} \mathrm{IL}_{-1} 7^{+}$(Tc17) cells were found to be decreased in mice exposed to $\triangle a l b 1$ conidia.

Tc17 cells are a unique subset of $\mathrm{CD}^{+} \mathrm{T}$ cells that have recently been associated with viral immunity (viral clearance), pulmonary inflammatory responses, patients with systemic lupus erythematosus, control of tumor growth, and contact dermatitis (40-44). Tc17 cells demonstrate functional plasticity, and are reported to produce proinflammatory cytokines and chemokines responsible for the enhanced recruitment of macrophages, natural killer cells, and neutrophils $(40,41,45)$. These cells have not been previously identified in models of fungal exposures, and may indicate a novel role for Tc17 cells in the immune responses to fungi. This decrease in Tc17 cells in the $\Delta a l b 1$ conidia exposed mice correlated with the significant decrease in neutrophils. 
In allergic asthma models, IL17 has been associated with inflammatory pulmonary responses. In the $\mathrm{C} 57 \mathrm{BL} / 6 \mathrm{~J}$ mice, eosinophil recruitment to the lungs was correlated to IL-17 production [48]. However, in a BALB/c OVA allergy model, IL-17 depletion resulted in increased pulmonary eosinophilia (46). The disparity between these studies is likely due to a combination of the genetic differences between $\mathrm{C} 57 \mathrm{BL} / 6 \mathrm{~J}$ and BALB/c strains and the exposure route or schedule, highlighting the importance of genetics and exposure protocols. Future experiments are needed to fully characterize the role of IL-17 in inflammatory cell recruitment in response to A. fumigatus exposures.

We also examined the ability of the mice to clear fungal spores from their lungs. Others have reported that $A$. fumigatus albino mutants are more rapidly phagocytosed and degraded than WT spores $(16,17,33)$. However, this had not been examined in an immunocompetent animal model of repeated exposures. We observed that the clearance of $\Delta a r p 2$ and $\Delta a l b 1$ conidia is more rapid than in mice exposed to WT at 5 and 24 hours post-challenge. After repeated fungal exposures, however, the ability to efficiently clear conidia was reduced at the 24 hour time point compared to single exposure mice, irrespective of melanin content. In a study by Murdock et al., it was reported that 24 hours after the final exposure, repeated exposures did not enhance conidial clearance, though clearance at earlier time points was not measured (47). Protective tolerance to fungi, regulated through IL-10 and $\mathrm{T}_{\text {Reg }}$ cells, is induced by repeated exposures to limit inflammation and subsequent tissue damage (48-50). The presence of IL-17 has also been shown to inhibit A. fumigatus clearance (51-53). In accordance with these studies, our decreased clearance results may correlate with the decreased $\mathrm{Tc} 17$ and airway neutrophil recruitment in response to $\Delta a l b 1$ conidia. It may be possible that the repeated exposures in our model result in the induction of a tolerance response to lessen the extent of inflammation, and subsequent tissue injury over time, thereby resulting in a higher numbers of conidia remaining in the lungs of adaptive mice. Future experiments to examine IL-10, IL-17, and $\mathrm{T}_{\text {Reg }}$ cell responses and the production kinetics following repeated exposures to melanin-deficient conidia would aid in determining the mechanisms affected by the presence or absence of melanin.

In summary, we have characterized the pulmonary immune response to repeated fungal exposures in an immunocompetent model and are among the first to have identified a Tc17 cell population following repeated A. fumigatus exposures. We have also shown that a lack of melanin in A. fumigatus conidia alters pulmonary immune responses (decreased Tc17 cell and 
increased eosinophil cell numbers) and enhances conidia clearance, yet does not appear to impact the induction of antibody or allergic sensitization. Although melanin has been shown to be an important virulence factor in invasive disease models, it appears to be less significant in disease pathogenesis in an immunocompetent exposure model. This is likely due to the efficiency of the intact immune system to clear most fungal conidia within 72 hours, regardless of their melanin content. Importantly, this is the first study reporting the presence Tc17 cells within the lungs in response to fungal exposures, thereby suggesting a novel role for these cells in the immune response to Aspergillus fumigatus conidia.

\section{ACKNOWLEDGEMENTS}

The authors thank Diane Schwegler-Berry for help with the preparation and analysis with FESEM and Michael Kashon for statistical analysis advice. The findings and conclusions in this report are those of the authors and do not necessarily represent the views of the National Institute for Occupational Safety and Health. This work was supported in part by an interagency agreement with the National Institute of Environmental Health Sciences (CDC IAA\#12-NS1201). 


\section{REFERENCES}

1. Eduard, W. 2009. Fungal spores: a critical review of the toxicological and epidemiological evidence as a basis for occupational exposure limit setting. Critical Reviews in Toxicology 39: 799-864.

2. Latge, J. P. 1999. Aspergillus fumigatus and aspergillosis. Clin Microbiol Rev 12: 310350.

3. Devereux, G. 2006. The increase in the prevalence of asthma and allergy: food for thought. Nat Rev Immunol 6: 869-874.

4. Simon-Nobbe, B., U. Denk, V. Pöll, R. Rid, and M. Breitenbach. 2008. The spectrum of fungal allergy. International Archives of Allergy and Immunology 145: 58-86.

5. Chaudhary, N., and K. Marr. 2011. Impact of Aspergillus fumigatus in allergic airway diseases. Clinical and Translational Allergy 1: 4.

6. Agarwal, R., A. N. Aggarwal, D. Gupta, and S. K. Jindal. 2009. Aspergillus hypersensitivity and allergic bronchopulmonary aspergillosis in patients with bronchial asthma: systematic review and meta-analysis The International Journal of Tuberculosis and Lung Disease 13: 936-944.

7. Latge, J. P. 2001. The pathobiology of Aspergillus fumigatus. Trends in Microbiology 9: 382-389.

8. Pihet, M., P. Vandeputte, G. Tronchin, G. Renier, P. Saulnier, S. Georgeault, R. Mallet, D. Chabasse, F. Symoens, and J.-P. Bouchara. 2009. Melanin is an essential component for the integrity of the cell wall of Aspergillus fumigatus conidia. BMC Microbiology 9: 177.

9. Brakhage, A. A., K. Langfelder, G. Wanner, A. Schmidt, and B. Jahn. 1999. Pigment biosynthesis and virulence. Contributions to Microbiology 2: 205-215.

10. Wheeler, M. H., and A. A. Bell. 1988. Melanins and their importance in pathogenic fungi. Current Topics in Medical Mycology 2: 338-387.

11. Jacobson, E. S. 2000. Pathogenic roles for fungal melanins. Clinical Microbiology Reviews 13: 708-717.

12. Tsai, H. F., R. G. Washburn, Y. C. Chang, and K. J. Kwon-Chung. 1997. Aspergillus fumigatus arp1 modulates conidial pigmentation and complement deposition. Molecular Microbiology 26: 175-183.

13. Jahn, B., F. Boukhallouk, J. Lotz, K. Langfelder, G. Wanner, and A. A. Brakhage. 2000. Interaction of human phagocytes with pigmentless Aspergillus conidia. Infection and Immunity 68: 3736-3739.

14. Volling, K., A. Thywissen, A. A. Brakhage, and H. P. Saluz. 2011. Phagocytosis of melanized Aspergillus conidia by macrophages exerts cytoprotective effects by sustained PI3K/Akt signalling. Cellular Microbiology 13: 1130-1148.

15. Thywißen, A., T. Heinekamp, H.-M. Dahse, J. Schmaler-Ripcke, S. Nietsche, P. F. Zipfel, and A. A. Brakhage. 2011. Conidial dihydroxynaphthalene melanin of the human pathogenic fungus Aspergillus fumigatus interferes with the host endocytosis pathway. Frontiers in Microbiology 2.

16. Tsai, H. F., Y. C. Chang, R. G. Washburn, M. H. Wheeler, and K. J. Kwon-Chung. 1998. The developmentally regulated alb1 gene of Aspergillus fumigatus: its role in modulation of conidial morphology and virulence. J Bacteriol 180: 3031-3038.

17. Langfelder, K., B. Jahn, H. Gehringer, A. Schmidt, G. Wanner, and A. A. Brakhage. 1998. Identification of a polyketide synthase gene (pksP) of Aspergillus fumigatus 
involved in conidial pigment biosynthesis and virulence. Medical Microbiology and Immunology 187: 79-89.

18. Chai, L. Y. A., M. G. Netea, J. Sugui, A. G. Vonk, W. W. J. van de Sande, A. Warris, K. J. Kwon-Chung, and B. Jan Kullberg. 2010. Aspergillus fumigatus conidial melanin modulates host cytokine response. Immunobiology 215: 915-920.

19. Huffnagle, G. B., G. H. Chen, J. L. Curtis, R. A. McDonald, R. M. Strieter, and G. B. Toews. 1995. Down-regulation of the afferent phase of $\mathrm{T}$ cell-mediated pulmonary inflammation and immunity by a high melanin-producing strain of Cryptococcus neoformans. J Immunol 155: 3507-3516.

20. Tsai, H. F., M. H. Wheeler, Y. C. Chang, and K. J. Kwon-Chung. 1999. A developmentally regulated gene cluster involved in conidial pigment biosynthesis in Aspergillus fumigatus. Journal of Bacteriology 181: 6469-6477.

21. Templeton, S. P., A. D. Buskirk, B. Law, B. J. Green, and D. H. Beezhold. 2011. Role of germination in murine airway CD8 T-cell responses to Aspergillus conidia. PLoS ONE 6: e18777.

22. Hettick, J., B. Green, A. Buskirk, M. Kashon, J. Slaven, E. Janotka, F. Blachere, D. Schmechel, and D. Beezhold. 2008. Discrimination of Aspergillus isolates at the species and strain level by matrix-assisted laser desorption/ionization time-of-flight mass spectrometry fingerprinting. Analytical Biochemistry 380: 276-281.

23. Rao, G. V., S. Tinkle, D. N. Weissman, J. M. Antonini, M. L. Kashon, R. Salmen, L. A. Batteli, P. A. Willard, M. D. Hoover, and A. F. Hubbs. 2003. Efficacy of a technique for exposing the mouse lung to particles aspirated from the pharynx. $J$ Toxicol Environ Health A 66: 1441-1452.

24. Stevens, W. W., T. S. Kim, L. M. Pujanauski, X. Hao, and T. J. Braciale. 2007. Detection and quantitation of eosinophils in the murine respiratory tract by flow cytometry. $J$ Immunol Methods 327: 63-74.

25. Foster, B., C. Prussin, F. Liu, J. K. Whitmire, and J. L. Whitton. 2007. Detection of intracellular cytokines by flow cytometry. In Curr Protoc Immunol. John Wiley and Sons, Inc.

26. Shvedova, A. A., E. Kisin, A. R. Murray, V. J. Johnson, O. Gorelik, S. Arepalli, A. F. Hubbs, R. R. Mercer, P. Keohavong, N. Sussman, J. Jin, J. Yin, S. Stone, B. T. Chen, G. Deye, A. Maynard, V. Castranova, P. A. Baron, and V. E. Kagan. 2008. Inhalation vs. aspiration of single-walled carbon nanotubes in C57BL/6 mice: inflammation, fibrosis, oxidative stress, and mutagenesis. American Journal of Physiology - Lung Cellular and Molecular Physiology 295: L552-L565.

27. Hettick, J., B. Green, A. Buskirk, M. Kashon, J. Slaven, E. Janotka, F. Blachere, D. Schmechel, and D. Beezhold. 2008. Discrimination of Penicillium isolates by matrixassisted laser desorption/ionization time-of-flight mass spectrometry fingerprinting. Rapid Communications in Mass Spectrometry 22: 2555-2560.

28. Hettick Justin, M., J. Green Brett, D. Buskirk Amanda, E. Slaven James, L. Kashon Michael, and H. Beezhold Donald. 2011. Discrimination of Fungi by MALDI-TOF Mass Spectrometry. In Rapid Characterization of Microorganisms by Mass Spectrometry. American Chemical Society. 35-50.

29. Buskirk, A. D., J. M. Hettick, I. Chipinda, B. F. Law, P. D. Siegel, J. E. Slaven, B. J. Green, and D. H. Beezhold. 2011. Fungal pigments inhibit the matrix-assisted laser 
desorption/ionization time-of-flight mass spectrometry analysis of darkly pigmented fungi. Analytical Biochemistry 411: 122-128.

30. Aimanianda, V., J. Bayry, S. Bozza, O. Kniemeyer, K. Perruccio, S. R. Elluru, C. Clavaud, S. Paris, A. A. Brakhage, S. V. Kaveri, L. Romani, and J.-P. Latge. 2009. Surface hydrophobin prevents immune recognition of airborne fungal spores. Nature 460: 1117-1121.

31. Lamhamedi-Cherradi, S.-E., R. E. Martin, T. Ito, F. Kheradmand, D. B. Corry, Y.-J. Liu, and M. Moyle. 2008. Fungal proteases induce Th2 polarization through limited dendritic cell maturation and reduced production of IL-12. The Journal of Immunology 180: 60006009.

32. Robinson, B. W., T. J. Venaille, A. H. Mendis, and R. McAleer. 1990. Allergens as proteases: an Aspergillus fumigatus proteinase directly induces human epithelial cell detachment. The Journal of Allergy and Clinical Immunology 86: 726-731.

33. Jahn, B., A. Koch, A. Schmidt, G. Wanner, H. Gehringer, S. Bhakdi, and A. A. Brakhage. 1997. Isolation and characterization of a pigmentless-conidium mutant of Aspergillus fumigatus with altered conidial surface and reduced virulence. Infect Immun 65: 5110-5117.

34. Paris, S., J.-P. Debeaupuis, R. Crameri, M. Carey, F. Charlès, M. C. Prévost, C. Schmitt, B. Philippe, and J. P. Latgé. 2003. Conidial Hydrophobins of Aspergillus fumigatus. Applied and Environmental Microbiology 69: 1581-1588.

35. Rivera, A., G. Ro, H. Van Epps, T. Simpson, I. Leiner, D. B. Sant'Angelo, and E. Pamer. 2006. Innate immune activation and $\mathrm{CD} 4+\mathrm{T}$ cell priming during respiratory fungal infection. Immunity 25: 665-675.

36. Wüthrich, M., G. S. Deepe, and B. Klein. 2012. Adaptive Immunity to Fungi. Annual Review of Immunology 30: 115-148.

37. Souwer, Y., K. Szegedi, M. L. Kapsenberg, and E. C. de Jong. 2010. IL-17 and IL-22 in atopic allergic disease. Current Opinion in Immunology 22: 821-826.

38. Korn, T., E. Bettelli, M. Oukka, and V. K. Kuchroo. 2009. IL-17 and Th17 Cells. Annual Review of Immunology 27: 485-517.

39. Carvalho, A., A. De Luca, S. Bozza, C. Cunha, C. D'Angelo, S. Moretti, K. Perruccio, R. G. Iannitti, F. Fallarino, A. Pierini, J.-P. Latgé, A. Velardi, F. Aversa, and L. Romani. 2012. TLR3 essentially promotes protective class I-restricted memory CD8+ T-cell responses to Aspergillus fumigatus in hematopoietic transplanted patients. Blood 119: 967-977.

40. Hamada, H., M. d. 1. L. Garcia-Hernandez, J. B. Reome, S. K. Misra, T. M. Strutt, K. K. McKinstry, A. M. Cooper, S. L. Swain, and R. W. Dutton. 2009. Tc17, a Unique Subset of CD8 T Cells That Can Protect against Lethal Influenza Challenge. The Journal of Immunology 182: 3469-3481.

41. Garcia-Hernandez, M. d. 1. L., H. Hamada, J. B. Reome, S. K. Misra, M. P. Tighe, and R. W. Dutton. 2010. Adoptive Transfer of Tumor-Specific Tc17 Effector T Cells Controls the Growth of B16 Melanoma in Mice. The Journal of Immunology 184: 4215-4227.

42. Henriques, A., L. Inês, M. Couto, S. Pedreiro, C. Santos, M. Magalhães, P. Santos, I. Velada, A. Almeida, T. Carvalheiro, P. Laranjeira, J. M. Morgado, M. L. Pais, J. A. P. d. Silva, and A. Paiva. 2010. Frequency and functional activity of Th17, Tc17 and other Tcell subsets in Systemic Lupus Erythematosus. Cellular Immunology 264: 97-103. 
43. Yeh, N., N. L. Glosson, N. Wang, L. Guindon, C. McKinley, H. Hamada, Q. Li, R. W. Dutton, P. Shrikant, B. Zhou, R. R. Brutkiewicz, J. S. Blum, and M. H. Kaplan. 2010. Tc17 Cells Are Capable of Mediating Immunity to Vaccinia Virus by Acquisition of a Cytotoxic Phenotype. The Journal of Immunology 185: 2089-2098.

44. Zhao, Y., A. Balato, R. Fishelevich, A. Chapoval, D. L. Mann, and A. A. Gaspari. 2009. Th17/Tc17 infiltration and associated cytokine gene expression in elicitation phase of allergic contact dermatitis. British Journal of Dermatology 161: 1301-1306.

45. Yen, H.-R., T. J. Harris, S. Wada, J. F. Grosso, D. Getnet, M. V. Goldberg, K.-L. Liang, T. C. Bruno, K. J. Pyle, S.-L. Chan, R. A. Anders, C. L. Trimble, A. J. Adler, T.-Y. Lin, D. M. Pardoll, C.-T. Huang, and C. G. Drake. 2009. Tc17 CD8 T Cells: Functional Plasticity and Subset Diversity. The Journal of Immunology 183: 7161-7168.

46. Hellings, P. W., A. Kasran, Z. Liu, P. Vandekerckhove, A. Wuyts, L. Overbergh, C. Mathieu, and J. L. Ceuppens. 2003. Interleukin-17 orchestrates the granulocyte influx into airways after allergen inhalation in a mouse model of allergic asthma. American Journal of Respiratory Cell and Molecular Biology 28: 42-50.

47. Murdock, B. J., N. R. Falkowski, A. B. Shreiner, A. A. Sadighi Akha, R. A. McDonald, E. S. White, G. B. Toews, and G. B. Huffnagle. 2012. Interleukin-17 Drives Pulmonary Eosinophilia following Repeated Exposure to Aspergillus fumigatus Conidia. Infection and immunity 80: 1424-1436.

48. Romani, L. 2004. Immunity to fungal infections. Nature Reviews. Immunology 4: 1-23.

49. Romani, L., and P. Puccetti. 2006. Protective tolerance to fungi: the role of IL-10 and tryptophan catabolism. Trends in Microbiology 14: 183-189.

50. Montagnoli, C., F. Fallarino, R. Gaziano, S. Bozza, S. Bellocchio, T. Zelante, W. P. Kurup, L. Pitzurra, P. Puccetti, and L. Romani. 2006. Immunity and tolerance to Aspergillus involve functionally distinct regulatory $\mathrm{T}$ cells and tryptophan catabolism. The Journal of Immunology 176: 1712-1723.

51. Werner, J. L., A. E. Metz, D. Horn, T. R. Schoeb, M. M. Hewitt, L. M. Schwiebert, I. Faro-Trindade, G. D. Brown, and C. Steele. 2009. Requisite role for the dectin-1 $\beta$-glucan receptor in pulmonary defense against Aspergillus fumigatus. The Journal of Immunology 182: 4938-4946.

52. Nembrini, C., B. J. Marsland, and M. Kopf. 2009. IL-17-producing T cells in lung immunity and inflammation. Journal of allergy and clinical immunology 123: 986-994.

53. Zelante, T., A. De Luca, C. D' Angelo, S. Moretti, and L. Romani. 2009. IL-17/Th17 in anti-fungal immunity: What's new? European Journal of Immunology 39: 645-648. 


\section{Chapter 3}

Development and Characterization of a Murine Model of Repeated Dry Fungal Exposure 


\section{INTRODUCTION}

Personal exposures to A. fumigatus are increasingly associated with a variety of adverse health outcomes including invasive aspergillosis, allergic sensitization, and asthma (1). The ubiquitous nature of this fungus in the environment, accompanied by the abundant production of small $(2-3 \mu \mathrm{m})$ aerodynamic conidia may result in personal exposure up to $10^{4}$ condia $/ \mathrm{m}^{3}$ (2). The concentration of fungal exposure may even be higher in water-damaged buildings and some occupational environments (2). In immunocompromised hosts, exposure to A. fumigatus often leads to invasive diseases with high mortality (3). In immunocompetent individuals, repeated exposures to low concentrations results in rapid innate clearance of conidia. However, over the past two decades, there has been a steady increase in reported fungal-induced allergies and asthma $(4,5)$. To date, the fungal-specific factors and immunological mechanisms that lead to the induction of these allergic diseases are largely unknown. It has been hypothesized that repeated exposures to high concentrations of A. fumigatus in immunocompetent individuals result in fungal persistence within the lung, which may cause and/or exacerbate hypersensitivity pneumonitis, allergy, and asthma.

Numerous murine models of fungal exposure have been developed to determine how $A$. fumigatus influences immune responses (6-22). These studies have provided insight into $A$. fumigatus immune responses, but the test articles have often consisted of uncharacterized extracts or liquid spore suspensions that do not resemble natural human exposures (6). The surface of conidia contains a number of potentially important immunostimulatory antigens that may be removed or diluted upon preparation of liquid suspensions. This may alter the interaction of conidia with immune cells, thereby modifying the subsequent immune response(s). Furthermore, high water content is necessary for A. fumigatus germination and suspending conidia in saline may prematurely activate the conidia (23). Activation of conidia significantly alters the surface structure and accessible antigenic molecules. Germination has been shown to significantly increase the expression of A. fumigatus antigens and allergens (24, 25). Germination also results in the production of several immunosuppressive mycotoxins that influence the immune response substantially (26-31). A. fumigatus conidia have also been shown to secrete a small toxin after short incubations in Hank's Balanced Salt Solution that is immunosuppressive and distinct from known mycotoxins (32). Since many of the immune mechanisms known to play a role in fungal immunity may be impacted by the liquid exposure 
method, it is vital to characterize the influence of dry fungal aerosols on the pulmonary immune responses, which is the primary aim of these studies. Thus, we hypothesize that these secreted antigens, allergens and mycotoxins produced during the preparation of fungal suspensions for instillation or aspiration exposures may significantly limit and confound previous murine models of A. fumigatus exposures.

To date, there have been limited animal studies completed to ascertain the influence of dry fungal exposures on the pulmonary immune response. Schuh et al. developed a dry-fungal aerosol exposure chamber and examined immune responses in mice that had previously been primed to induce allergy $(33,34)$. In this model, mice were sensitized, both subcutaneously and intraperitoneally, a single time with a commercial $A$. fumigatus fungal extract that contained a heterogenous mixture of proteins from both conidia and hyphae using alum as an adjuvant. Mice were then exposed to the extract, intranasally, once per week for three weeks. Following these exposures, mice were either exposed a single time to a suspension of A. fumigatus conidia via intratracheal instillation, or were subjected to inhalation exposure(s) up to three times at different intervals following the first inhalation exposure (34). A. fumigatus aerosols were created by directing a stream of air across a fungal culture plate. The inhalation exposures required mice to be anesthetized and subjected to nose-only exposures for ten minutes. The total number of conidia that the mice were exposed to was quantified post-exposure. Interestingly, these studies reported multiple differences, including an increase in eosinophils and lymphocytes, antibody responses, and overall lung inflammation to dry-aerosols when compared to liquid involuntary aspiration. The authors noted that dry inhalation exposures exacerbated the allergic asthma phenotype (34).

Although these studies provide support for our overall hypothesis that dry fungal exposures will result in similar, yet qualitatively different immune responses when compared to aspiration exposures, there are several limitations associated with their model. The studies presented in this chapter examined the ability of dry conidia to affect the immune response in pre-sensitized mice, thereby prematurely skewing the results toward a Th2 response. Further, the concentration of conidia was not able to be altered during exposures, and the exposure concentration was determined following sacrifice of the animal following exposure. These studies also required animals to be anesthetized during the exposures, thereby altering the normal respiration rate and tidal volume of the mice. The following experiments address these caveats 
through use of a multi-animal $(\mathrm{n}=15)$, nose-only, acoustical generator exposure system that allows for real-time analysis of particle size, deposition estimations, and manipulation of exposure concentrations. Furthermore, naïve female BALB/c mice were used in these studies to characterize the immune responses following repeated exposures to dry A. fumigatus conidia.

The secondary aim of these studies was to identify phenotypic characteristics that may impact allergy/asthma induction to further aid in characterizing the immune responses to dry WT A. fumigatus conidia. Melanin, a large macromolecular pigment that gives A. fumigatus conidia the characteristic blue-green color, is a virulence factor that protects the fungus from innate clearance $(35,36)$. Several studies, using melanin knock-down A. fumigatus mutants, have shown that albino conidia are more rapidly cleared from the lungs and are less virulent than the wild-type (WT) conidia in a murine model of invasive aspergillosis (37, 38). Melanin has also been shown to protect WT conidia from opsonization by the complement pathway, as less C3 molecules bind to the surface of WT than albino conidia (39). Wild-type conidia are also able to quench reactive oxygen species (ROS) produced by innate macrophages and neutrophils (40-42). The presence of melanin within the conidial wall has also been shown to inhibit phagolysosome acidification, and inhibit host cell apoptosis, thereby providing an environment for the conidia to sequester until germination occurs $(43,44)$. Additionally, albino conidia have been shown to induce greater proinflammatory cytokine production, including TNF $\alpha$ and IL-6, which does not occur following exposure to WT conidia (45). These results indicate that the presence of melanin sufficiently protects WT conidia from innate clearance, allowing for fungal persistence and germination within the lungs. Therefore, we hypothesize that melanin may play a role in the induction of A. fumigatus-induced allergy and asthma.

In this study, we sought to develop an immunocompetent murine model of repeated pulmonary exposures to A. fumigatus conidia that more closely resembles natural environmental fungal exposure. Through use of this model, we characterized the immune response following exposure and examined the impact of fungal melanization on the induction of an allergic phenotype. To do this, a unique, tightly-controlled, nose-only acoustical generator exposure system was developed. With this system, several parameters can be accurately monitored in realtime, including the: 1) number of particles that are aerosolized, 2) size distribution of the bioaerosol (2-5 $\mu \mathrm{m}$ in diameter for A. fumigatus conidia), and 3) calculated estimate in the number of conidia that are deposited in the lungs. To determine if fungal melanization has a 
functional role in the development of fungal-induced allergy or asthma, albino conidia obtained from a point mutation in the melanin synthesis pathway mutation, was derived from a clinical isolate of A. fumigatus were used. These studies are among the first to characterize the pulmonary immune responses following repeated dry fungal exposures in a competent murine model which more closely resembles natural exposures.

\section{MATERIALS AND METHODS}

\section{Fungal culture}

Fungal strains (A. fumigatus B-5233/ATCC 13073 - the wild-type (WT) parent strain and Aalb1) were received as a gift from Dr. June Kwon-Chung (NIAID, Bethesda, MD). Fungal cultures were grown for 14 days at $25^{\circ} \mathrm{C}$ on malt extract agar. A modified method was used to grow conidia for acoustical generator experiments (46). Briefly, $200 \mathrm{ml}$ of dry brown rice (Mahatma, Allentown, PA) was autoclaved for 30 minutes at $121^{\circ} \mathrm{C}$. Sterilized, distilled, deionized water $(10 \mathrm{ml})$ was added to one malt extract plate, and conidia were suspended through agitation with a sterile inoculating loop. The fungal suspension was then used to inoculate to the autoclaved rice, and $100 \mathrm{~mL}$ of additional sterile water was also added to completely submerge the rice. The rice/A. fumigatus suspension was stirred and decanted from the rice into a sterile $50 \mathrm{ml}$ conical tube. Test MEA plates were also prepared from the fungal suspension to ensure there were no contaminant sources. Approximately 10-12 g of rice was added to sterile $100 \mathrm{~mm}$ petri dishes, and $4 \mathrm{ml}$ of the decanted fungal suspension was added to each plate. Plates were wrapped in parafilm and incubated at $25^{\circ} \mathrm{C}$ for $10-14$ days prior to use. Plates were shaken daily to dislodge rice aggregates.

\section{Animals}

Female BALB/c mice, aged 5-7 weeks (Jackson Laboratory, Bar Harbor, ME), were acclimated for approximately one week before initial exposures. The mice were housed in filtered, ventilated polycarbonate cages in groups of five on autoclaved hardwood chip bedding. The temperature in the animal facility was maintained between $68^{\circ} \mathrm{F}$ and $72^{\circ} \mathrm{F}$ and the relative humidity between $36 \%$ and 57\%. The light/dark cycle was maintained on 12-hour intervals. Mice were provided with NIH-31 modified 6\% irradiated rodent diet (Harlan Teklad) and tap water ad libitum. Sentinel mice were free of viral pathogens, parasites, mycoplasmas, 
Helicobacter, and CAR. The NIOSH animal facility is an environmentally controlled barrier facility that is fully accredited by the Association for the Assessment and Accreditations of Laboratory Animal Care International. All animal procedures were performed under a NIOSH Animal Care and Use Committee approved protocol 12-BG-M-003.

\section{Animal Exposures}

A nose-only inhalation exposure system was developed to expose mice to unaltered $A$. fumigatus conidia. Air from a water seal compressor was conditioned by passing it through a dryer, a charcoal filter, and then a high efficiency particulate air (HEPA) filter. The air stream was then run through a mass flow controller (GFC37, Aalborg, Orangeburg, NY) that regulated the air flow $(6 \mathrm{~L} / \mathrm{min})$ in the exposure chamber (Figure 3.1A). The conditioned air flowed through a modified PITT-3 acoustical aerosol generator. The acoustical generator consists of a speaker covered with a rubber membrane, upon which rice cultures were placed and allowed to settle 12-24 hours prior to exposure. The generator used the acoustical energy of a speaker to vibrate the conidia laden rice. This resulted in the detachment, deaggregation and aerosolization of the spores within the generator. The air-conidia mixture was then passed into a modified noseonly exposure chamber (Intox Products, LLC, Moriarty, NM). The conidia entered the top of the chamber into a column that spanned the chamber's height. Animals were positioned in separate pods that projected out radially from the column. The conidia entered the pods through a port directly in front of the nose of the animals. Exhaled air exited via radial ports around the nose of the mouse and into a second column where the air was HEPA filtered and fed into the house exhaust. The nose-only unit could house up to 24 pods, but only 15 mice were exposed at each sampling interval. Two additional pods served as sample ports. The first sample port was used to gravimetrically measure the mass concentration of the conidia during an exposure. The second sample port was connected to a light scattering device (DataRAM4, ThermoElectron Co., Franklin, MA) that provided a real-time estimate of the mass concentration of the conidia in the exposure chamber. Additionally, the DataRAM also measured the system temperature and humidity. During test runs, the gravimetric sample port was used to collect samples for electron microscopy and to size the particles with an aerodynamic particle sizer (APS, TSI Inc., Shoreview, MN). The resulting micrographs and APS measurements confirmed that the spores retained morphological attributes and largely consisted of a single spore suspension consisting of 
amerospores $(2-4 \mu \mathrm{m})$. The mass concentration estimates derived from the DataRAM were also shown to correlate with the number of spores within the exposure chamber (APS measurements).

A lung deposition model was developed based on mice deposition measurements from Raabe et al. (47). This allowed for estimation in the number of conidia deposited within the murine lung given the size distribution of the fungal aerosols. A scale factor was established and applied to the DataRAM signal that estimated the concentration of conidia deposited in the mouse lung per unit time. The integrated value of deposited conidia was calculated throughout murine exposures and the system automatically turned off the aerosol generator when the target conidial concentration was reached ( 100 min). Custom software was developed by Travis Goldsmith to provide a graphical user interface to monitor, record, and change exposure variables. The conidial mass concentration was regulated during exposure intervals through the use of software feedback loops. During exposures, control animals were placed in a similar control chamber which resided next to the fungal exposure chamber. All environmental conditions were kept constant for control animals.

Animals were pre-bled via tail vein nick prior to exposure. Mice were also acclimated to the exposure chamber by placing them in the nose-only housing units for increasing time, up to two hours, over the course of two weeks prior to the first exposure. The aim of this protocol was to reduce the stress conditions that may result from the prolonged restraint required for the fungal exposures. For exposures, fifteen mice per time point were placed in the control chamber (exposed to HEPA filtered air only) or were exposed to WT, or $\Delta a l b l$ conidia. Mice were placed individually in nose-only exposure units attached to the acoustical generator for approximately 2 hours until real-time particle counts reached $5 \times 10^{5}$ conidia. Mice were exposed twice per week (3-4 days apart) for 4 total weeks, and sacrificed at 4, 24, 48, or 72 hours post-final exposure via intraperitoneal injection of sodium pentobarbital (Sleepaway®, Fort Dodge Animal Health, Fort Dodge, IA). Sera were also collected from each mouse at the time of sacrifice via cardiac puncture.

\section{Field emission scanning electron microscopy of aerosolized conidia}

A sample of 14 day old rice cultures was aerosolized, and A. fumigatus conidia were sampled onto a polycarbonate filter from the nose-only chamber. The sample was air dried for three days, attached to an aluminum mount with double-stick carbon tape, and sputter coated 
with gold/palladium. Images were collected on a Hitachi (Tokyo, Japan) S-4800 field emission scanning electron microscope.

\section{Modified local lymph node assay}

Mice were exposed to $5 \times 10^{3}, 1 \times 10^{4}, 1 \times 10^{5}$, or $1 \times 10^{6}$ WT conidia or HEPA filtered air only via the acoustical generator exposure chamber once daily for three days, and then rested for two days. To serve as a positive control for exposures, a group of mice were exposed to 200 $\mu \mathrm{g}$ of hyphal extract via pharyngeal aspiration using the same exposure schedule. On the sixth day, mice were injected i.v. via the lateral tail vein with $20 \mu \mathrm{Ci}{ }^{3} \mathrm{H}$-thymidine (Dupont $\mathrm{NEN}$, Waltham, MA; specific activity $2 \mathrm{Ci} / \mathrm{mmol})$. Five hours following ${ }^{3} \mathrm{H}$-thymidine injection, animals were euthanized via $\mathrm{CO}_{2}$ inhalation. The mediastinal lymph nodes, located at the bifurcation of the bronchi in the lung, were removed for analysis. Lymph nodes were homogenized between frosted microscope slides, cell suspensions were prepared, and samples were incubated with $5 \%$ trichloroacetic acid overnight at $4^{\circ} \mathrm{C}$. Samples were then mixed with scintillation fluid and counted using a Tri-Carb 2500TR liquid scintillation counter. Stimulation indices (SIs) were calculated by dividing the mean disintegrations per minute (DPM) by the mean DPM obtained from the control chamber exposure mice. Pre- and post-weight data was additionally recorded to assess potential toxicity. Concentrations of conidia that induced greater than $10 \%$ weight loss were not considered for the repeated exposures, despite the SI value.

\section{Airway reactivity assessment}

Plethysmography experiments were included to determine non-specific airway reactivity at 24 and 48 hours following the final conidia exposure $(n=8)$. Eight control and 8 exposed mice were examined at both 24 and 48 hour time intervals. Briefly, mice were placed in individual units and allowed to acclimate for 5-10 minutes prior to taking a baseline respiratory reading. Baseline values were collected for 5 minutes, followed by the addition of $10 \mathrm{mg} / \mathrm{ml} \mathrm{of}$ methacholine that was added to the nebulizer and exposed to the mice for 3 minutes. Following a 2 minute recovery, mice were then exposed to $25 \mathrm{mg} / \mathrm{ml}$ and $50 \mathrm{mg} / \mathrm{ml}$ methacholine, during which time data was continuously collected. Enhanced respiratory pause $(\mathrm{PenH})$ values were collected as an average value calculated over the 5 minute course of exposure to each methacholine (Mch) concentration. 
Histology

From each A. fumigatus strain used for exposure, three mice per time point were sacrificed at 4, 24, 48, or 72 hours post final exposure. Samples for histology were prepared as indicated in Chapter 2. Tissue processing, embedding, hematoxylin and eosin (H\&E) and Grocott's Methenamine Silver (GMS) staining was performed by the West Virginia University Tissue Bank (Morgantown, WV).

Swollen and germinating conidia in GMS slides data were acquired by counting the number of conidia visualized over 100 different fields of view (40X magnification), covering the all lobes of both lungs. Conidia 2-3X the size of resting conidia were classified as swollen. Data are presented as the total conidial counts over exposure duration or the percentage of conidia that are germinating ((swollen conidial counts + germ tube counts)/ total number of conidia counted).

\section{Collection of BALF and mediastinal lymph nodes}

BALF collection was completed as indicated in Chapter 2 and prepared for flow cytometric analysis. Following centrifugation of the BALF at $860 \mathrm{x} g$ for 2 minutes, the supernatant was retained and frozen at $-20^{\circ} \mathrm{C}$ for complement analysis. Following collection of BALF, the mediastinal lymph nodes were removed from each mouse and suspended in $1 \mathrm{ml}$ of sterile PBS. These samples were then homogenized by grinding the nodes between frosted microscope slides. The lymph node samples were centrifuged for 3 minutes at $860 \mathrm{x} g$ and the supernatant was discarded. Cells isolated from the lymph node were prepared for flow cytometric analysis.

\section{Flow Cytometric Analysis: Differential cell staining}

All reagents were obtained from BD Biosciences (BD Biosciences, San Jose, CA) unless otherwise specified. BALF cell composition was determined by flow cytometric analysis of recovered lavage cells in suspension. BALF was centrifuged for 3 minutes at $860 \mathrm{x} \mathrm{g}$, the supernatant discarded, and the cell pellet resuspended and washed in $1 \mathrm{ml}$ of FACS buffer (PBS, $5 \%$ fetal bovine serum, and $0.05 \%$ sodium azide). The washed pellet was resuspended, and divided in half for cell enumeration and intracellular cytokine staining. One half of the cells were stained in FACS buffer, 10\% rat serum, Fc-receptor blocking antibody (clone 24G2) and the following antibodies at 1:100: rat anti-mouse Ly-6G, rat anti-mouse Siglec-F, pan-leukocyte rat 
anti-mouse CD45, and rat anti-mouse CD11c. Mediastinal lymph nodes were also surface stained, to identify B cell populations, in FACS buffer, 10\% rat serum, Fc-receptor blocking antibody (clone 24G2), and B220/CD45R and IgE antibodies. After staining 30 minutes on ice in the dark, cells were washed with FACS buffer and fixed with BD Cytofix for 10 minutes. Cells were then washed and resuspended in FACS buffer for flow cytometry. Populations of cells were evaluated by flow cytometric analysis on a BD LSRII equipped with FACSDiva software(BD Biosciences, San Jose, CA). Cells were collected for one minute or until 50,000 events were captured. Neutrophils were defined as CD45 ${ }^{\text {hi }}$ Ly- $6 G^{\text {hi }} C D 11 c^{\text {low }}$, eosinophils as Ly$6 \mathrm{G}^{\text {low }}$ SiglecF $\mathrm{F}^{\text {hi }} \mathrm{CD} 11 \mathrm{c}^{\text {low }}$, and alveolar macrophages as $\mathrm{Ly}-6 \mathrm{G}^{\text {low }}$ Siglec $\mathrm{F}^{\text {hi }} \mathrm{CD} 11 \mathrm{c}^{\text {hi }}$, as previously reported (48). Total cell numbers were quantified using acridine orange nuclear staining and an automated cell counter (Cellometer AutoX4, Nexcelom Bioscience, Lawrence, MA). Total numbers of each cell population were obtained by multiplying the frequency of specific population by the total number of BALF cells recovered for each animal.

\section{Flow cytometric analysis: Intracellular cytokine staining}

BALF T-cells were quantified using rat anti-mouse CD8 and CD4 antibodies. T-cell cytokine production was determined by fluorescent intracellular cytokine staining (ICS) as previously described (49). Briefly, the BALF and mediastinal lymph node suspensions were centrifuged for three minutes at $2000 \mathrm{xg}$ and washed in $1 \mathrm{ml}$ of RPMI 1640 complete medium (GIBCO, Grand Island, NY) supplemented with 10\% fetal calf serum, Pen/Strep/Glutamine, and $0.05 \% \beta$-mercaptoethanol. The supernatant was discarded and a solution of Leukocyte Activation Cocktail with GolgiPlug in $0.2 \mathrm{ml}$ complete medium was added to each sample for stimulation of cytokine production and simultaneous inhibition of cytokine secretion. Cells were incubated at $37^{\circ} \mathrm{C}$ in $5 \% \mathrm{CO}_{2}$ for four hours. Following incubation, the BALF cells were washed in FACS buffer and stained for flow cytometry analysis using rat anti-mouse CD4, rat antimouse CD8, rat anti-mouse B220/CD45R, and rat anti-mouse CD25 on ice in the dark for 30 minutes. Mediastinal lymph node cells were stained using rat anti-mouse CD3e, rat anti-mouse CD4, and rat anti-mouse CD8 antibodies. Cells were then washed in FACS buffer and centrifuged, and the cell pellets were resuspended in BD Cytofix/Cytoperm for 10 minutes to allow for fixation and permeabilization required for subsequent ICS. Cells were washed with 1 $\mathrm{ml}$ of BD PermWash, and resuspended in PermWash. Each BALF sample was stained with rat 
anti-mouse IFN- $\gamma$, rat anti-mouse IL-13, rat anti-mouse IL-5, rat anti-mouse IL-22, rat antimouse IL-10, and rat anti-mouse IL-17A (eBioscience, San Diego, CA). Mediastinal lymph nodes were stained with rat anti-mouse IL-13, Armenian hamster anti-mouse IL-9 (Biolegend, San Diego, CA), rat anti-mouse IL-10, rat anti-mouse IL-12, rat anti-mouse IL-22, rat anti-mouse IL-17A, and rat anti-mouse IFN- $\gamma$. All antibody concentrations were 1:100. Cell populations were analyzed on a BD LSRII, with lymphocytes gated on the basis of low forward and side scatter, then subsequently gated on $\mathrm{CD}^{+}$or $\mathrm{CD}^{+}$populations to determine intracellular expression of cytokines.

\section{Preparation of conidial protein extracts}

Conidial extracts, from WT, $\triangle a r p 2$, and $\triangle a l b 1$, were obtained from 14 day old MEA plates grown as indicated in Chapter 2. Conidia were harvested from the plates by adding $5 \mathrm{ml}$ of sterile, distilled, deionized water, and agitating the surface of the agar with a sterile inoculating loop. Samples were frozen overnight at $-80^{\circ} \mathrm{C}$, and then lyophilized for 3-5 days to remove the suspension solution. Glass beads $(0.5 \mathrm{~mm})$ were added to each sample. Conidia were then subjected to 3 x 1 minute bead beating cycles using a mini-bead beater (BioSpec, Bartlesville, OK). Sodium bicarbonate buffer $(\mathrm{pH} 8.0,2 \mathrm{ml})$, containing $0.5 \mathrm{mM}$ EDTA and protease inhibitors (Complete Mini, Roche Diagnostics, Indianapolis, IN), was added and the samples and then agitated by three one-minute bead-beating cycles, centrifuged, and the resulting supernatant was used for SDS-PAGE. Protein concentrations were estimated using $1.5 \mu \mathrm{l}$ of extract for NanoDrop analysis at $580 \mathrm{~nm}$ (NanoDrop, Wilmington, DE).

\section{Western blot analysis}

Conidial extracts $(30 \mu \mathrm{g})$ were mixed with Laemmli's (Bio-Rad, Hercules, CA) sample buffer and heated at $95^{\circ} \mathrm{C}$ for 5 minutes. Samples were loaded in individual lanes (30 $\mu \mathrm{g} /$ well) and resolved using a $12 \%$ polyacrylamide gel with a $4 \%$ stacking gel run at $100 \mathrm{~V}$ for $60-90$ minutes as done in Chapter 2. Proteins were then transferred to a $0.2 \mu \mathrm{m}$ nitrocellulose membrane at $16 \mathrm{~V}$ overnight at $4^{\circ} \mathrm{C}$. The membrane was then blocked for 1 hour with $3 \%$ bovine serum albumin (BSA) in Tris-buffered saline (TBS). Pooled polyclonal sera from exposed mice was then diluted to the indicated concentration (1:200 or 1:500) in 3\% BSA/TBS and incubated with the membrane at room temperature for 1 hour with gentle agitation. The blot was then 
washed 3 times with TBS/Tween and incubated with alkaline phosphatase (AP)-conjugated goat anti-mouse IgG heavy and light chain (Promega, Madison, WI), or goat anti-mouse APconjugated IgM (Calbiochem, Billerica, MA) diluted 1:5000 in 3\% BSA/TBS for 1 hour at room temperature with gentle agitation, washed. The blot was then washed 3 times with TBS/Tween and developed using the alkaline phosphatase substrate, Pierce 1-Step NBT/BCIP Solution (Thermo Scientific, Rockford, IL).

\section{Total IgE ELISA}

Total IgE concentrations in pre-bleed sera and pooled polyclonal sera isolated from exposed mice were determined using a total IgE kit, as per the manufacturer's instructions (Biolegend, San Diego, CA). Sera were prepared at 1:10 and serially diluted, and plates were read at 450 and $570 \mathrm{~nm}$. Background absorbance values obtained from the $570 \mathrm{~nm}$ readings were subtracted from the $450 \mathrm{~nm}$ values. The standard curve was created from known concentrations $(0.156 \mathrm{ng} / \mathrm{ml} \mathrm{-} 10 \mathrm{ng} / \mathrm{ml})$ of $\operatorname{IgE}$ standards, and unknown IgE sample concentrations were extrapolated from the curve.

\section{Data analysis methods}

Analysis of flow cytometric samples was performed with BD FACSDiva software (BD Biosciences, San Jose, CA). GraphPad Prism was used for generation of graphs and statistical analysis for clearance and conidial germination experiments, and total IgE levels (GraphPad Software, La Jolla, CA). The data were analyzed via two way analysis of variance (ANOVA) followed by Bonferroni post-test. Statistical analysis of cellularity and intracellular cytokine producing cells was performed using SAS version 9.3 for Windows (SAS, Cary, NC). The data were log transformed prior to analysis and Proc Mix was used to run a two-way factorial analysis of variance (Fungal Exposure by Time). Pairwise comparisons were performed using Fishers Least Significant Difference test. Differences between experimental groups were considered significant with p-values less than 0.05 . 


\section{RESULTS}

Preliminary acoustical generator experiments

A new exposure system was developed to enable characterization of the immune responses following fungal exposures to dry fungal conidia. The acoustical generator, based on the Pitt-3 generator, was used in these studies and has previously been shown to aerosolize carbon nanotubes (50). Figure 3.1A shows a schematic of the dry particle generator system. The fungal aerosol is directed into a nose-only chamber where a DataRAM, particle counter and particle sizer are attached. To determine if the acoustical generator was capable of producing aerosolized A. fumigatus conidia in sufficient concentrations, funga/rice cultures were aerosolized. The real-time particle counts showed that greater than $1 \times 10^{8}$ conidia were produced (data not shown). Air samples from the exposure chamber were directed onto a polycarbonate filter and analyzed via field emission scanning electron microscopy to visually examine the generated aerosol. Images showed single and, in some cases, aggregate conidia were aerosolized and entered the nose-only chamber (Figure 3.1C). DataRam analysis, showing the size distribution of the particles, confirmed these results (Figure 3.1B). Importantly, no aerosolized rice grain fragments or hyphal fragments were observed in the electron microscopy images. These findings demonstrated that pure conidial aerosols were produced following acoustical generation. 
Figure 3.1 Preliminary dry particle generator exposure chamber
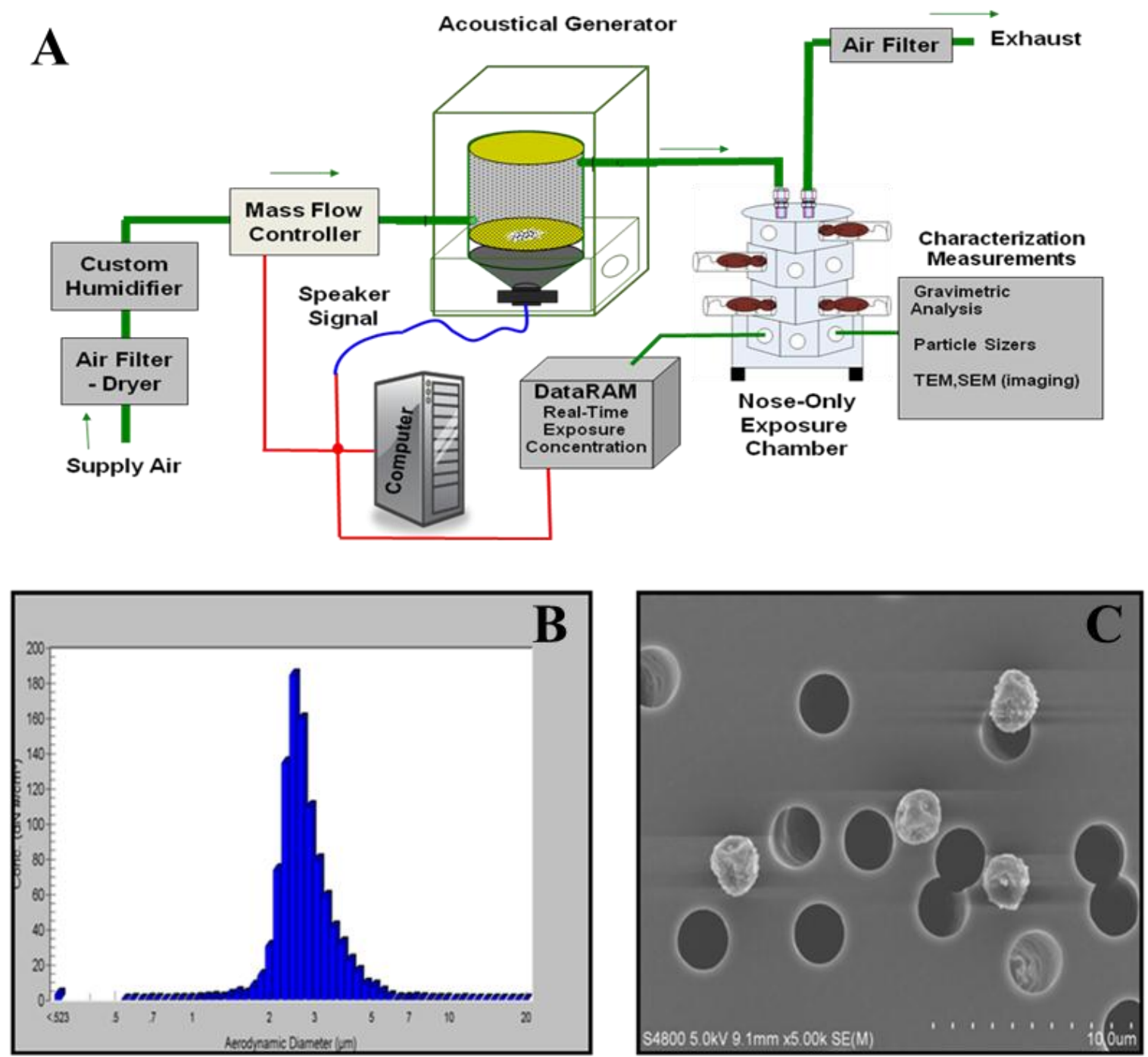

Figure 3.1: Preliminary dry particle generator exposure chamber experiments. A) Illustration showing the acoustical generator nose-only exposure chamber. Supply air is HEPA filtered and directed into the acoustical generator. The acoustical generator is then sent a signal to vibrate at a designated frequency which results in the formation of fungal aerosols from rice grains. The fungal aerosol is then directed into a nose-only chamber and air is filtered before being sent into the exhaust system. A real-time particle counter attached to the computer calculates the concentration of fungal particles being deposited into the airways, and the DataRAM reports that number to the computer, which can be altered during the exposure to obtain the desired deposition concentration. B) The aerosol particle size distribution produced by the acoustical generator. 2-5 $\mu \mathrm{m}$ is the size of single or aggregate A. fumigatus conidia. C) Field emission scanning electron microscopy image of A. fumigatus fungal conidia deposited on a polycarbonate filter placed in the nose-only chamber. 
To determine if generated conidia reached the terminal airways following nose-only exposures, as estimated by Rabbe et al., mice were exposed to WT conidia a single time and sacrificed immediately following exposure (47). Histopathological assessment showed fungal conidia lining the upper respiratory tract and deposited as deep as the smaller airways (Figure 3.2). These experiments demonstrated the applicability of the acoustical generator to create dry fungal aerosols which resulted in the deposition of conidia within the lower respiratory tract in murine nose-only exposures. 
Figure 3.2 Histology showing conidial deposition

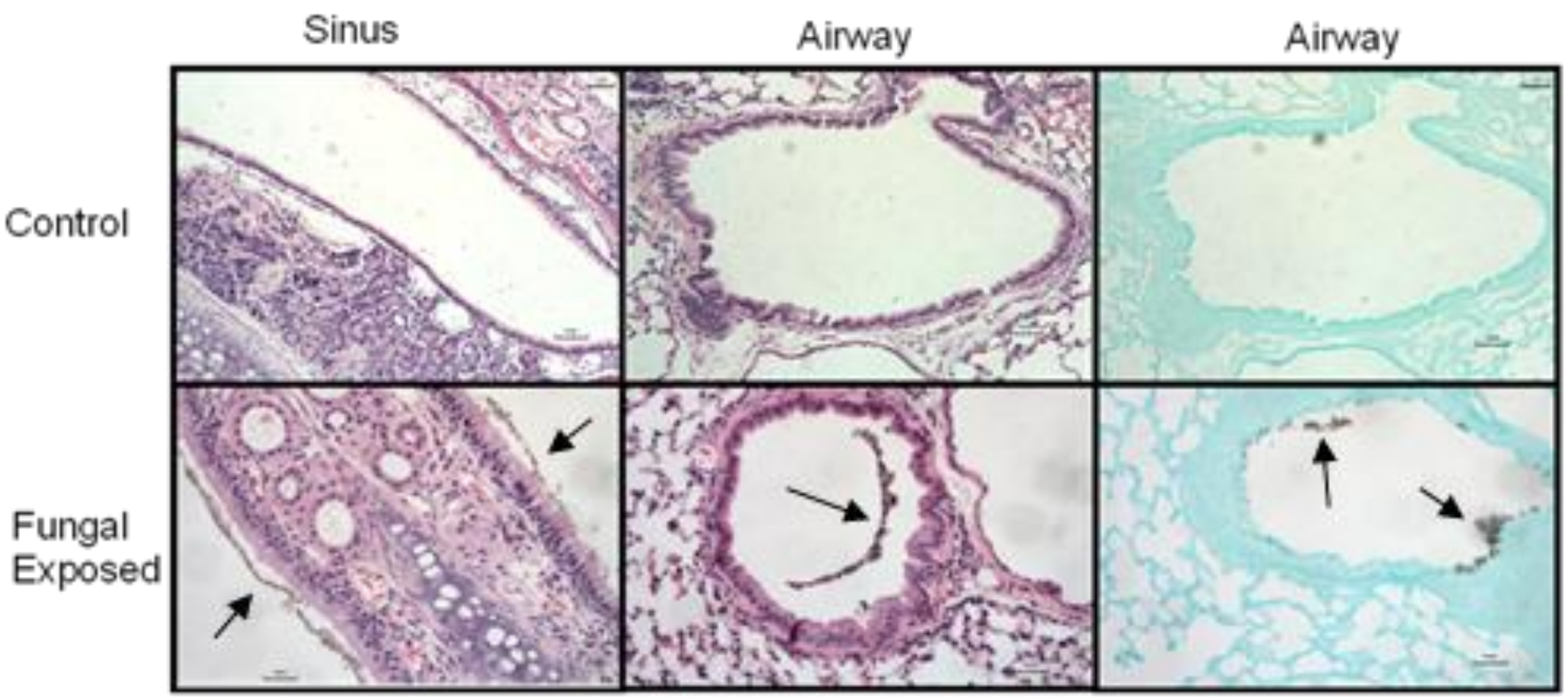

Figure 3.2: Histology (20X) of conidial deposition in the sinuses and airways following a single 3.5 hour exposure to $1 \times 10^{6}$ conidia (estimated using the deposition model) using the acoustical generator nose-only chamber. The left and middle panels include H\&E stained sections while the far right panels are GMS stained sections to resolve fungal particles. Conidia observed in the lining of the sinuses, lumen of the airways, and airways of fungal exposed mice (silver particulate in GMS indicated by arrows) indicating the deposition of fungal particles within the lungs. 
A modified local lymph node assay was conducted to determine if repeated A. fumigatus WT exposures induce proliferation of lymphocytes in the mediastinal lymph nodes. Additionally, this data was used to determine the optimal dose for future repeated exposure experiments. A. fumigatus hyphal extracts were used as a positive control due to the high concentration of known proteins/allergens associated with hyphae (as described in Chapter 1).

Four groups of mice were exposed to different concentrations $\left(5 \times 10^{3}, 1 \times 10^{4}, 1 \times 10^{5}\right.$, or $1 \times 10^{6}$ estimated lower airway deposition) of WT conidia using the acoustical generator. As shown in figure 3.3, lymphocyte proliferation was dose dependent, with $1 \times 10^{6}$ conidia (calculated deposition concentration) inducing the highest stimulation index ( 100 SI units). Mice that were exposed to this dose experienced greater than $10 \%$ weight loss over the course of the experiment. As a result of the toxicity associated with this exposure dose, $1 \times 10^{6}$ conidia were not used for future experiments. Exposures to less than $1 \times 10^{6}$ conidia did not induce weight loss and were less toxic when compared to $1 \times 10^{6}$ conidia doses. As a result, $5 \times 10^{5}$ conidia were chosen for further experiments. This concentration corresponds to the elevated levels described in human exposures that often occur in contaminated indoor or occupational environments (2). 
Figure 3.3 Local Lymph Node Assay

A. fumigatus Conidia-Induced

Bronchial Lym phocyte Proliferation

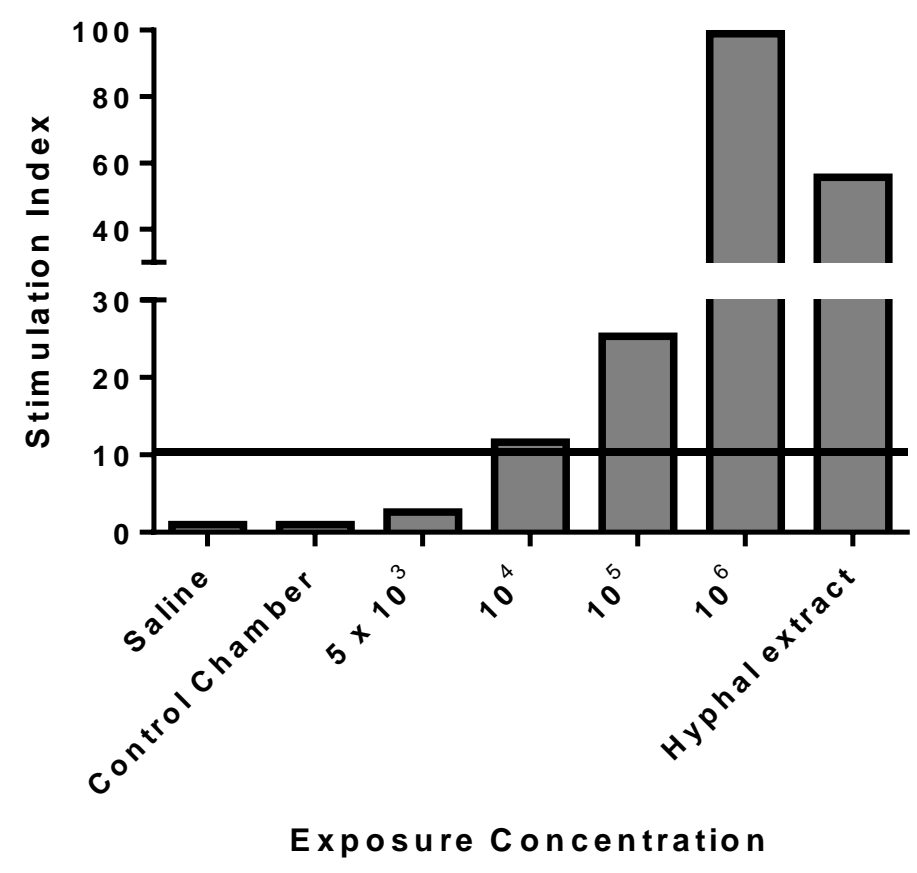

Figure 3.3. Average stimulation indices determined by a modified local lymph node assay. Mice were exposed to the indicated deposition concentration of WT conidia. Stimulation indices (SI) were calculated as average disintegration per minute of the exposed group/average disintegration per minute control group. Horizontal line at SI of 10 is an arbitrary index used to determine positive lymphocyte proliferation in these studies. $N=10$ mice/group. 


\section{Airway reactivity}

Following determination of the optimal exposure concentration, mice were exposed to dry aerosols of WT or $\Delta a l b 1$ conidia twice per week for 4 weeks. Antigen-specific airway reactivity was examined immediately following exposure using Buxco analysis; however, no reactivity was observed. Non-specific airway reactivity following methacholine challenge was examined at 24 and 48 hours post-final exposure. The results presented in Figure 3.4 show that each fungal strain induced an increased $\mathrm{PenH}$ value, which is indicative of an allergic/asthmatic phenotype. Interestingly, mice exposed to melanized wild-type fungi had peak PenH values at 48 hours post-final exposure, while exposures to albino conidia resulted in peak PenH at 24 hours (Figure 3.4). These results demonstrate that the presence of melanin in WT A. fumigatus conidia likely results in a delayed allergic airway response. 


\section{Figure 3.4 Methacholine-induced airway reactivity}
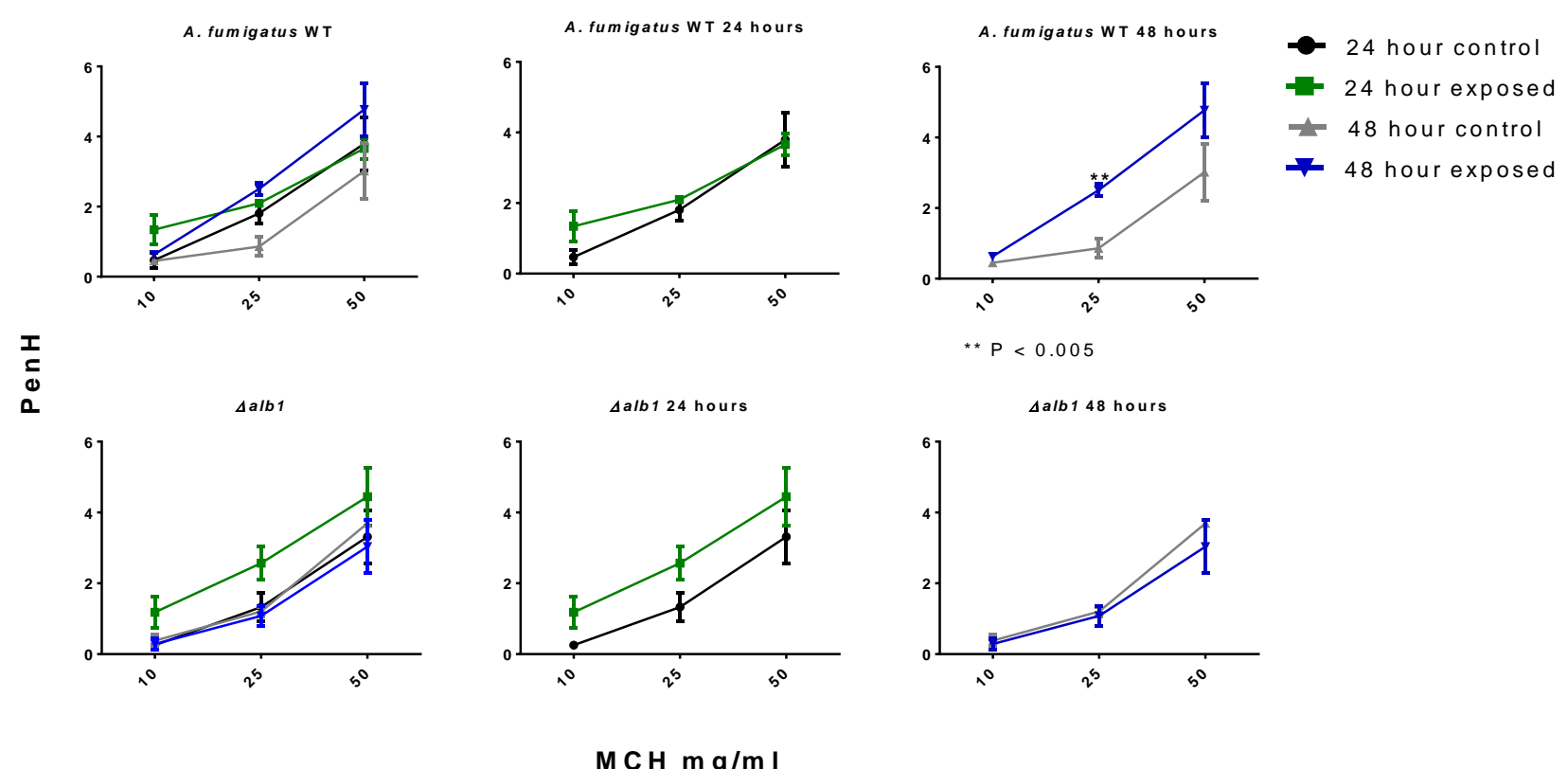

MCH $\mathbf{m g} / \mathbf{m ~ I ~}$

Figure 3.4: Methacholine-induced airway reactivity. Mice ( $n=4$ mice/group/time point) were exposed dry aerosolized conidia 8 times as indicated. Analysis was completed either 24 or 48 hours after the final exposure. Top) dry WT conidia, Bottom)) dry $\Delta$ alb1 conidia. Left panels-24 and 48 hour values, middle panels- 24 hour values only, right panels-48 hour values only. 


\section{Histopathology}

In order to examine the pathology associated with repeated exposures to dry conidia, cross sections of exposed lungs were examined at 4, 24, 48, and 72 hours following the final exposure. As shown in figure 3.5, within 4 hours of exposure to WT conidia, there is an increase in inflammatory cells within the lungs. Severe inflammation was evident, which predominantly affected the bronchioles (Figure $3.5 \mathrm{~A}$ and B). The inflammation was primarily characterized by the presence of macrophages and giant cell formation. Airway remodeling and fibrosis were also observed. Further, there was a marked increase in mucus production and goblet cell hyperplasia. By 24 hours, cellular infiltration was largely composed of neutrophils and eosinophils that surrounded the bronchioles, mucus production had begun to resolve (Figure $3.5 \mathrm{~A}$ and $\mathrm{B}$ ), and granulomas were observed near the larger airways (data not shown). These results were not dependent on melanin content. Within 4 hours of exposure, conidia had been transported from the airways into the interstitium (Figure 3.5A) and swollen conidia were observed in GMS stained slides (Figure 3.5 A, B, and C). Minimal germ tube formation was present at this time point in mice exposed to WT conidia (Figure $3.5 \mathrm{~A}$ and $\mathrm{C}$ ). Importantly, fewer intact conidia were evident in the lungs of mice exposed to albino conidia, even at 4 hours (Figure $3.5 \mathrm{~B}$ and C). By 24 and 48 hours, germination of WT conidia was significantly increased compared to albino conidia, which had failed to germinate (Figure $3.5 \mathrm{C}$ ). By 48 and 72 hours, most inflammation had resolved and few conidia remained despite melanin content (Figure 3.5).

Two mice (out of 60) that were exposed to WT conidia additionally developed torticollis, a condition characterized by a head tilt and uncontrollable spinning when picked up by the tail. GMS stained cross sections of the middle/inner ear of control chamber, asymptomatic WT exposed, and symptomatic WT exposed mice showed macrophage and neutrophil-mediated infiltration and conidia/germination only in symptomatic animals (Appendix A5). These preliminary data suggest that torticollis may result from A. fumigatus WT-induced inner ear infection, a condition likely caused by conidia that deposit in the Eustachian tubes following exposure. 
Figure 3.5 Histopathology

A

Control
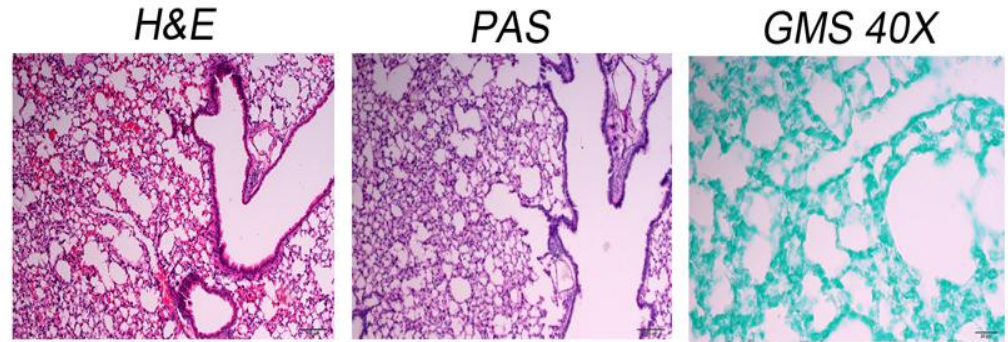

GMS 80X
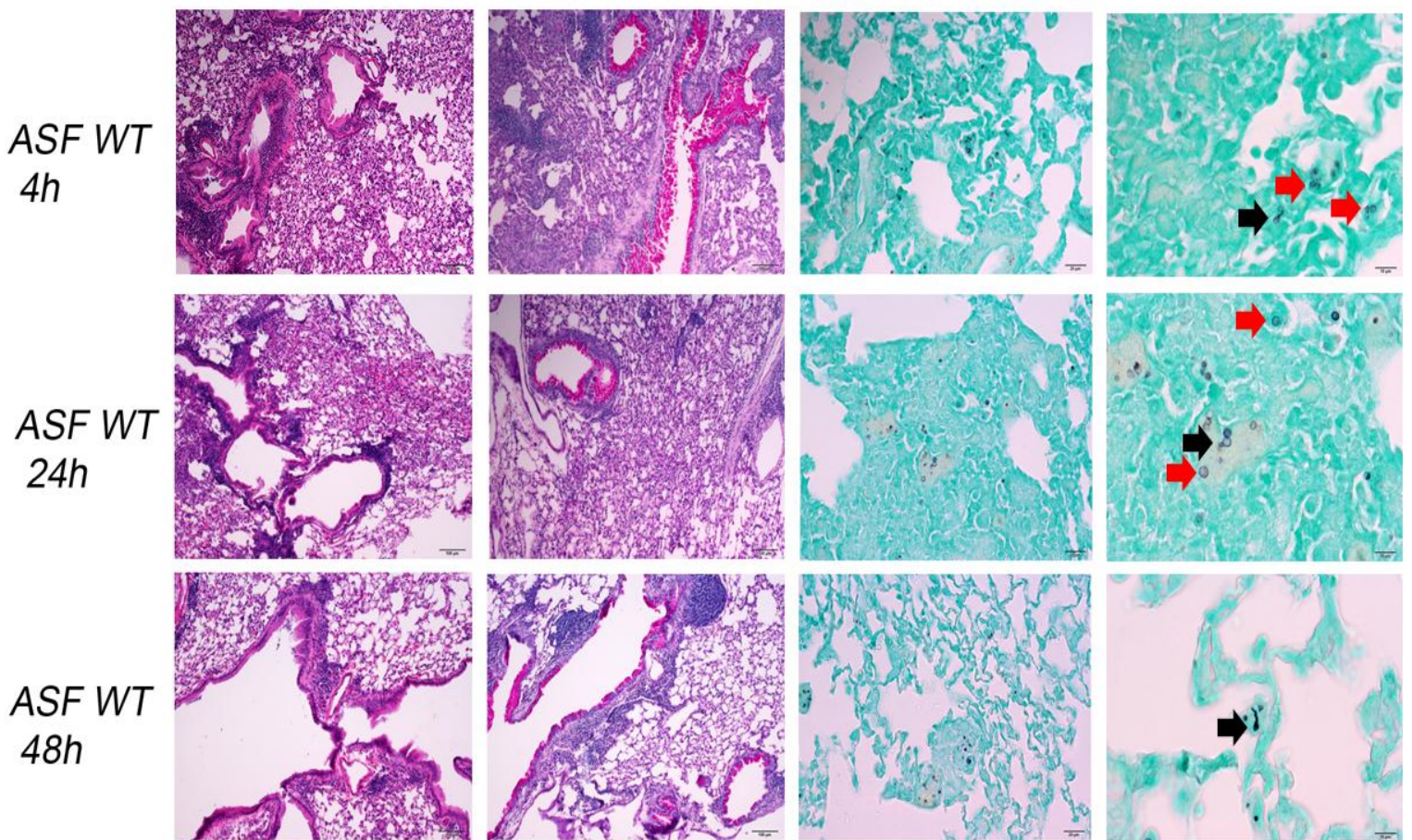

ASF WT $72 h$
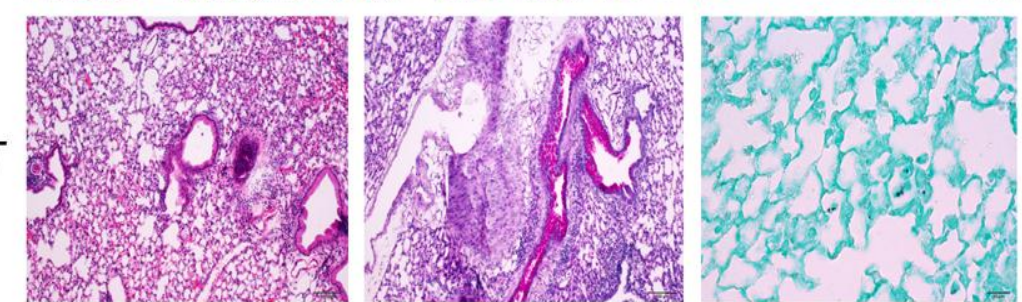


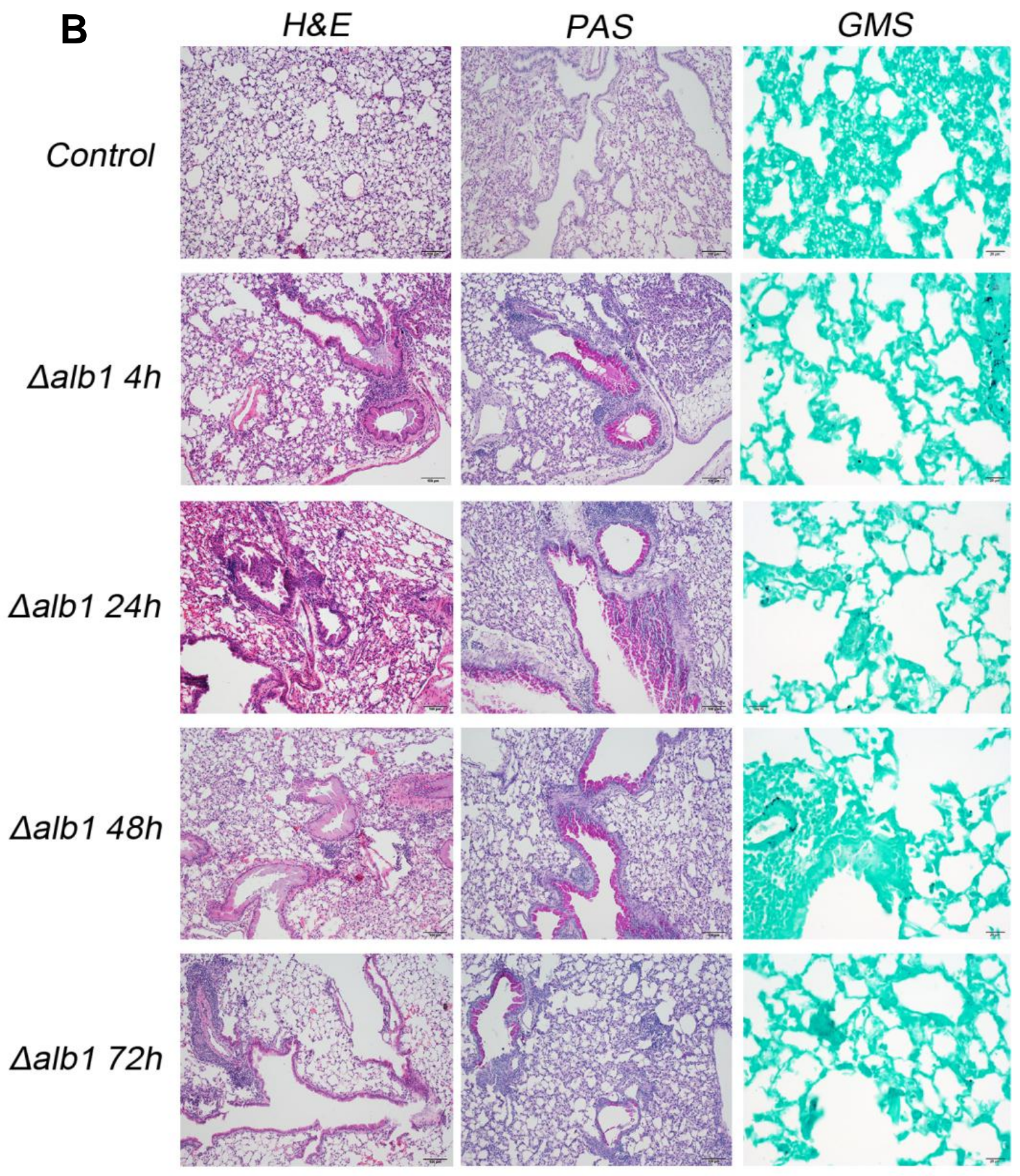



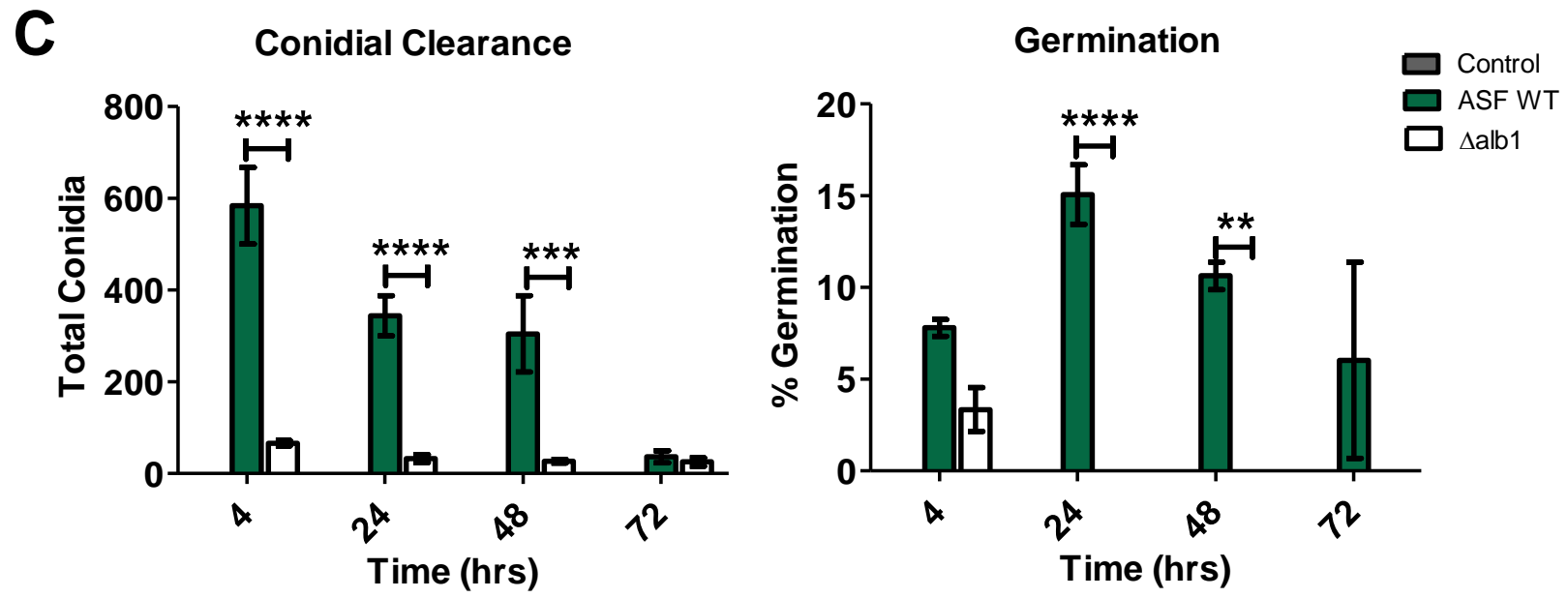

Figure 3.5 Histopathology of sections derived from WT and $\Delta a l b 1$ mice at $4 \mathrm{~h}$ and $24 \mathrm{~h}$. Representative histopathology sections from A) WT exposed mice or B) Aalb1 exposed mice sacrificed at the indicated time points. Left panels-H\&E stained sections at $10 X$ magnification, Middle panels-PAS stained sections at 10X magnification, and Right panels-GMS stained sections at 40X magnification. Red arrow heads indicate swollen conidia, Black arrow heads indicate germination. C) Quantification of conidia and germination (swollen conidia + germ tube formation) over time. Values were obtained by quantifying the number of conidia visualized at $40 \mathrm{X}$ across 100 random fields of view covering both lungs. Conidia were considered swollen when the size was $2-3 X$ that of resting conidia. ${ }^{* * * * P} \leq 0.0001,{ }^{* * *} P \leq 0.001,{ }^{* *} P \leq 0.01$.

\section{Complement}

The complement pathway is an innate mechanism that aids in the clearance of $A$. fumigatus conidia. However, complement has not been previously quantified following dry $A$. fumigatus conidial exposures and melanin has been shown to impact the deposition of complement proteins. In this experiment, we attempted to quantify the concentration of complement protein $\mathrm{C} 3$ (the converging point for all three complement pathways) following exposure in the BALF supernatant via ELISA. However, the concentration of $\mathrm{C} 3$ components in the BALF supernatant was below the detectable limit of the assay and could not be determined. 


\section{BALF Cellularity}

Quantification of total BALF cell numbers using flow cytometry showed a statistically significant difference in the cellular influx between the different exposure groups over time. Mice exposed to WT conidia had significantly higher B cell, macrophage, neutrophil, and eosinophil counts in the BALF 48 hours after the final exposure. Furthermore, neutrophils remained significantly higher in WT exposed mice by 72 hours (Figure 3.6). Compared to WT conidia, BALF cellularity in mice exposed to $\triangle a l b 1$ conidia was generally decreased and peaked for many cell types at 4 hours (Figure 3.6). These responses observationally appeared to berelated to conidia germination, as the highest numbers in each cell type occurred 48 hours post exposure to WT conidia and germination was elevated at this time point. These results also suggest that the presence of fungal melanin in WT conidia may have resulted in delayed immune cell recruitment to the airway. Interestingly, a downward shift in the alveolar macrophage population was also observed, and was more pronounced in WT exposed mice. These data show a downward shift in the population suggesting there is an accumulation of macrophages with different phenotypes/polarization, which could be classically-activated and alternativelyactivated macrophages (Appendix A4). 
Figure 3.6 BALF cellularity

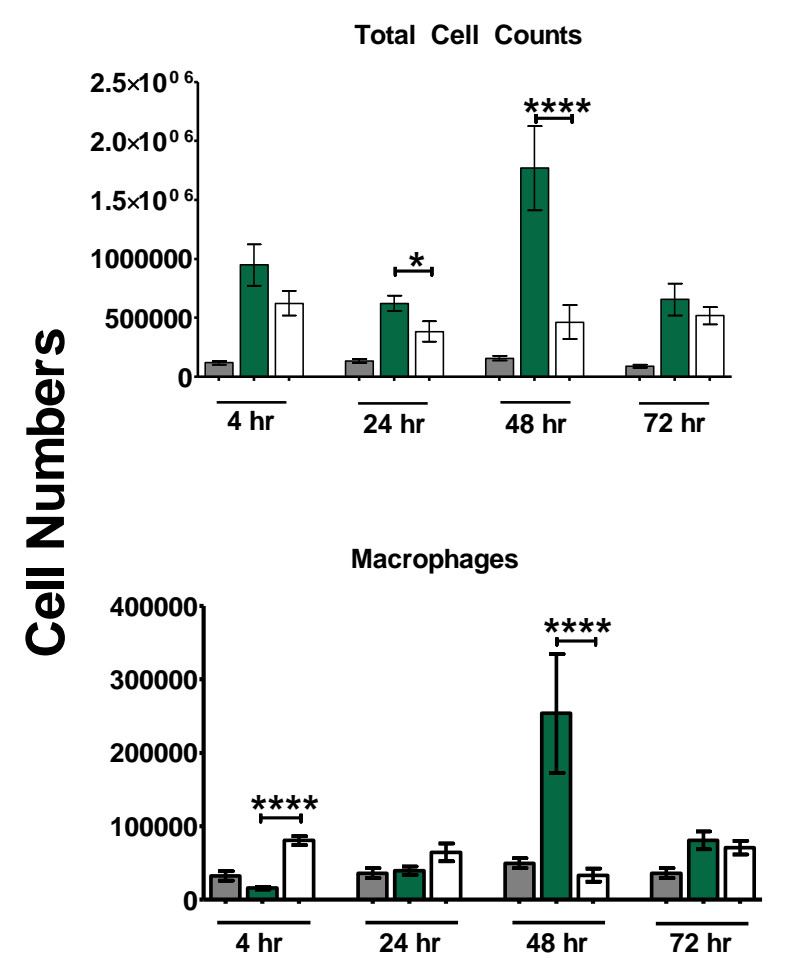

\section{BALF Cellularity}
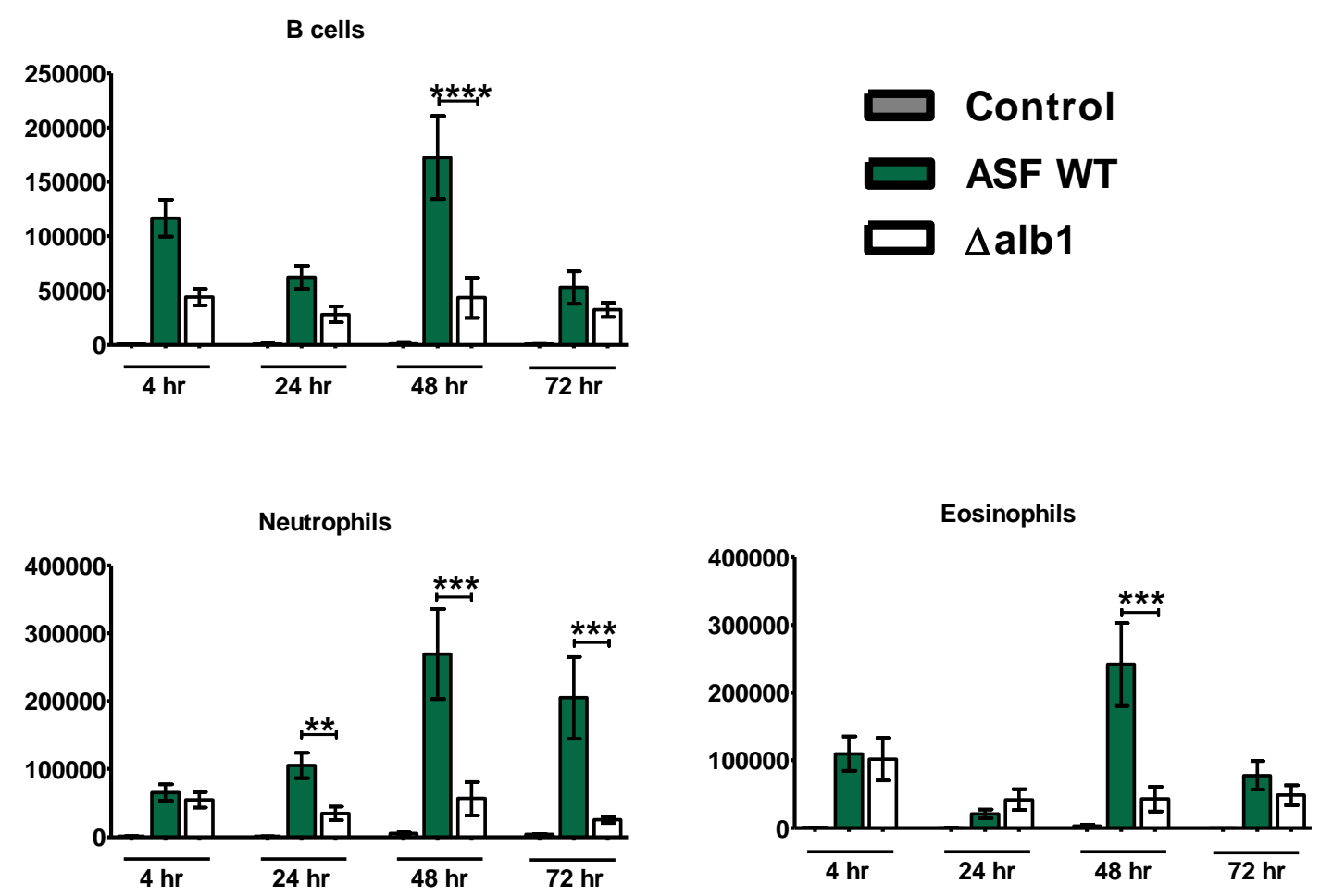

\section{Exposure Groups}

Figure 3.6: Total cell counts in the BALF. Total cell numbers were obtained through acridine orange staining and quantified using an automated cell counter. Following 8 dry conidial exposures, mice were sacrificed at the indicated time points to determine the kinetics of the cellular influx to the lung. (Control-n=30 miceltime point, Exposed $n=7-10$ micelgroup/time point). $* * * * P \leq 0.0001, * * * P \leq$ $0.001, * * P \leq 0.01, * P \leq 0.05$. 
To characterize the immune responses that occur following dry conidial exposures, intracellular cytokine staining on the BALF cells was performed. CD4 T cell responses were significantly higher at 48 hours following WT exposures; however, the levels of these cells were similar at each time point in mice exposed to albino conidia. Interestingly, the $\mathrm{T}_{\mathrm{H}} 1$ type $\mathrm{CD} 4{ }^{+} \mathrm{IFN}-\gamma^{+}$and $\mathrm{T}_{\mathrm{H}} 2 \mathrm{CD} 4^{+} \mathrm{IL}-13^{+} \mathrm{T}$ cells were significantly higher at 24 and 72 hours postexposure in mice exposed to albino conidia (Figure 3.7). Mice exposed to WT conidia recruited significantly more $\mathrm{CD} 4^{+} \mathrm{IL}-17^{+} \mathrm{T}$ cells to the airways at 48 hours compared to the albino group (Figure 3.7). Similar to the responses observed for each cytokine, $\mathrm{CD} 4^{+} \mathrm{TNF} \alpha^{+}$and $\mathrm{CD} 4{ }^{+} \mathrm{IL}-10^{+}$ populations were higher at 4 and 48 hours than at 24 and 72 hours in mice exposed to WT conidia (Appendix A6). While $\mathrm{CD}^{+} \mathrm{TNF}^{+}$levels were not assessed in response to albino conidia, $\mathrm{CD} 4^{+} \mathrm{IL}-10^{+}$numbers remained similar at each time point (Appendix A6). Aside from the $\mathrm{CD} 4^{+} \mathrm{IL}-17^{+} \mathrm{T}$ cells, the remaining CD4 $\mathrm{T}$ cell populations that were examined did not appear to be affected by germination.

Exposures to WT conidia resulted in peak CD8 T cells at 24 hours which decreased over time, while exposures to albino conidia induced a reduced response (Figure 3.7). Immediately following exposure, there were variable responses as shown by the significantly increased levels of $\mathrm{CD}^{+} \mathrm{IFN}_{-} \gamma^{+}$and elevated $\mathrm{CD}^{+} \mathrm{TNF}^{+}$and $\mathrm{CD} 8^{+} \mathrm{IL}-13^{+} \mathrm{T}$ cells at 4 hours (Figure 3.7 and Appendix A6). $\mathrm{CD}^{+} \mathrm{IL}-10^{+} \mathrm{T}$ cell numbers increased as levels of $\mathrm{CD} 8^{+} \mathrm{IFN}-\gamma^{+}$and $\mathrm{CD} 8^{+} \mathrm{TNF}^{+}$ declined (Figure 3.7, Appendix A6). Interestingly, $\mathrm{CD}^{+} \mathrm{IL}_{-} 17^{+} \mathrm{T}$ cells (Tc17) mirrored the total CD8 $\mathrm{T}$ cells present in the BALF at 24 hours, and were the predominant $\mathrm{T}$ cell present at this time point (Figure 3.7). These results corresponded to the kinetics of germination as shown in figure $3.5 \mathrm{C}$, suggesting that $\mathrm{Tc} 17$ cells may specifically respond to and have a function role in the clearance of germinating conidia (Appendix A9). Compared to repeated control aspiration exposures to silicone beads, these results were specific for A. fumigatus and not the result of innate particulate clearance (Appendix A8). 
Figure 3.7 BALF T Cells

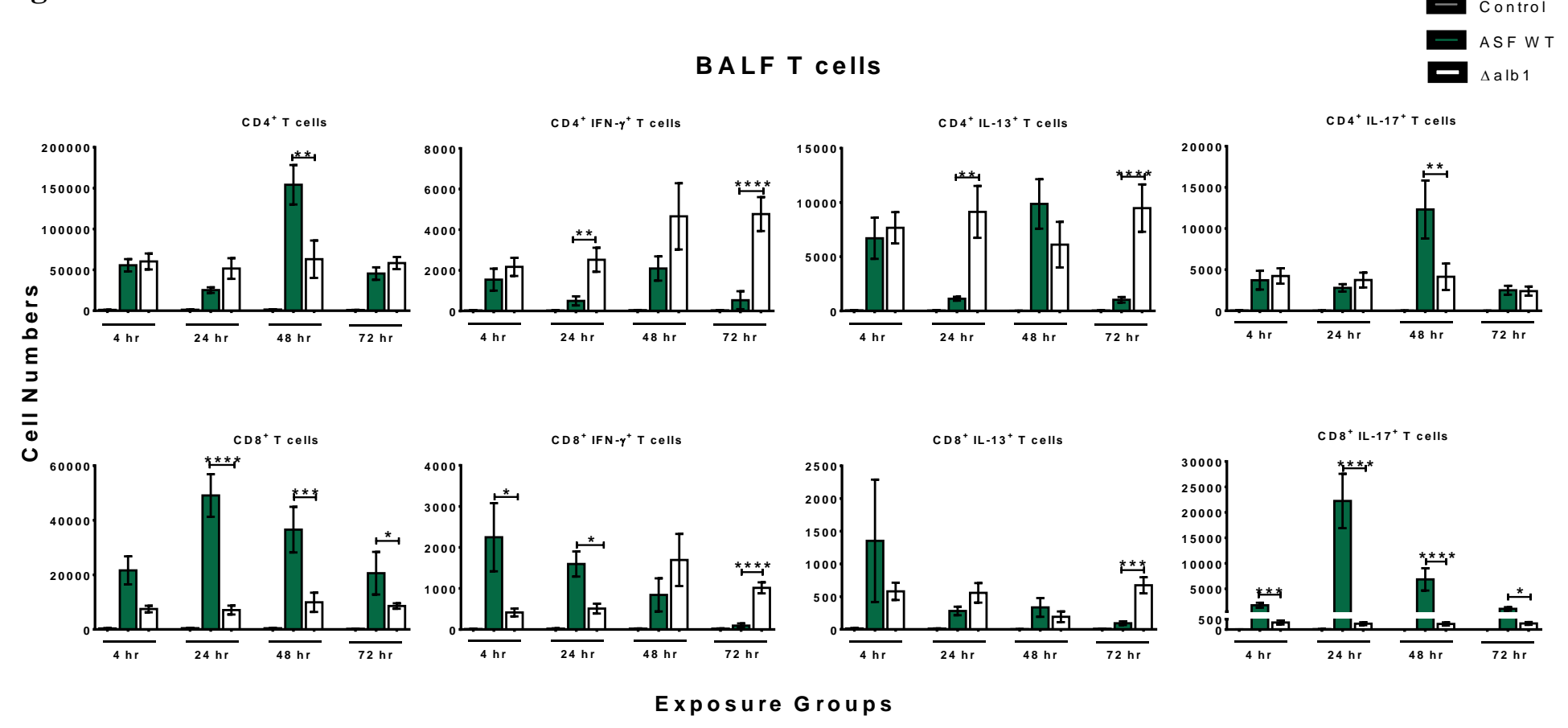

Figure 3.7. Intracellular cytokine flow cytometric analysis of the BALF. CD4 and CD8 T cells were quantified by multiplying the frequency of each individual cell population by the total cell counts. Mice were sacrificed at the indicated time points after the 8th exposure. Data are presented as the average \pm standard error of measure. $N=7-10$ mice/group per time point. $* * * * P \leq 0.0001$, $* * * P$ $\leq 0.001,{ }^{*} * P \leq 0.01, * P \leq 0.05$ 


\section{Mediastinal Lymph Nodes Cellularity}

The total mediastinal lymph node cell counts shown in Figure 3.8 are similar to those reported for each time point in the BALF analysis. In response to WT exposures, the number of lymph node and B cells steadily increased over time with significantly greater cell numbers observed at 48 and 72 hours, respectively (Figure 3.8). However, significantly higher lymph node and $\mathrm{B}$ cell counts, with elevated $\mathrm{IgE}^{+} \mathrm{B}$ cells, were observed at 4 hours in the albino exposure group. These counts were reduced at 24 hours, and began increasing again at 48 hours (Figure 3.8). The sharp decrease in total cell counts, but not B cells, was also observed at 72 hours in mice exposed to WT conidia (Figure 3.8). 
Figure 3.8 Total cell counts in the mediastinal lymph nodes

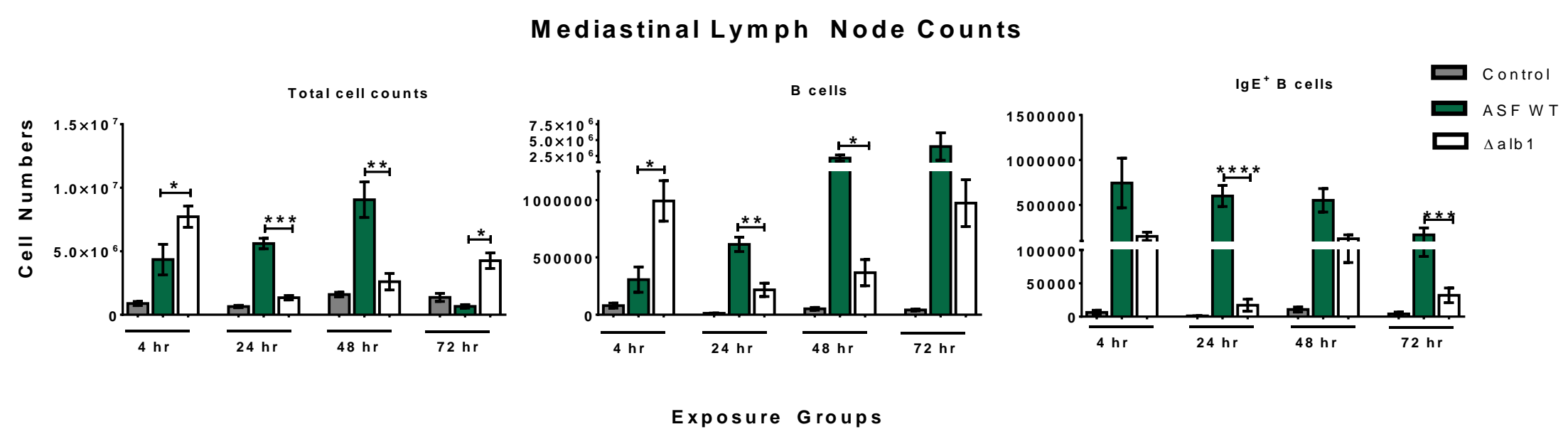

Figure 3.8: Total cell counts in the mediastinal lymph nodes. Total cell numbers were obtained through acridine orange staining and quantified using an automated cell counter. Following 8 dry conidial exposures, mice were sacrificed at the indicated time points to determine the kinetics of the cellular influx to the lung-associated nodes. (Control- $n=30$ miceltime point, Exposed $n=7-10$ mice/group/time point). $* * * * P \leq 0.0001, * * * P \leq 0.001, * * P \leq 0.01, * P \leq 0.05$. 
Intracellular cytokine staining was also performed on these samples for flow cytometric analysis. CD4+ $\mathrm{T}$ cells were significantly increased at 4 hours in mice exposed to albino conidia. However, WT exposures resulted in significantly more CD4 $\mathrm{T}$ cells at 24 hours. The $\mathrm{T}_{\mathrm{H}} 1$ $\mathrm{CD} 4^{+}$IFN- $\gamma+$ and $\mathrm{T}_{\mathrm{H}} 2 \mathrm{CD}^{+} \mathrm{IL}_{-}-13^{+}$and $\mathrm{IL}-9^{+}$populations were also significantly elevated in the albino exposure groups at 4 hours. This mixed response was delayed in the WT exposure group and observed at 24 hours (Figure 3.9 and Appendix A7). While similar patterns were observed in $\mathrm{CD} 4^{+} \mathrm{IL}-10^{+}$cells, these results were not significant. Importantly, these responses did not appear to be dependent on conidial germination and did not resemble the CD4 $\mathrm{T}$ cell responses observed in the BALF.

LN CD8 T cell responses were also analyzed (Figure 3.9). In the albino exposure group, a significant increase in $\mathrm{CD}^{+} \mathrm{IL}-13^{+}, \mathrm{CD} 8^{+} \mathrm{IL}-9^{+}$, and $\mathrm{CD} 8^{+} \mathrm{IL}-10^{+} \mathrm{T}$ cell responses occurred at the 4 hour time point (Figure 3.9 and Appendix A7). Further, $\mathrm{CD}^{+} \mathrm{IFN}^{-} \boldsymbol{\gamma}^{+}$counts were greatest at 48 hours and remained significantly higher at 72 hours in mice exposed to albino conidia compared to WT. $\mathrm{CD} 8{ }^{+} \mathrm{IFN}-\gamma^{+}$responses peaked at 24 hours and decreased over time as well in mice exposed to WT conidia, although this response was not statistically significant. $\mathrm{T}_{\mathrm{H}} 2$ cell populations were elevated in the WT exposure group at 24, 48, and 72 hours post exposure when compared to the albino group (Figure 3.9). $\mathrm{CD} 8{ }^{+} \mathrm{IL}-17^{+}$responses also increased with each time interval, and reached maximal levels at 72 hours following the final exposure to WT conidia (Appendix A7). Overall, these cell numbers were reduced compared to the total LN counts observed in Figure 3.8. These data did not correlate to the CD8 counts obtained from the BALF fluid, as peak responses were observed at 48 hours in the BALF and 72 hours in the LN. 
Figure 3.9 Intracellular cytokine flow cytometric analysis of the mediastinal lymph nodes

\section{Mediastinal Lymph Node T cells}

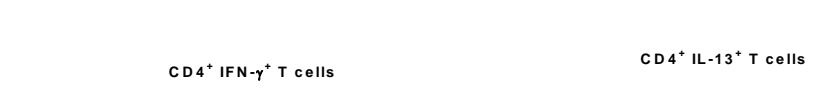

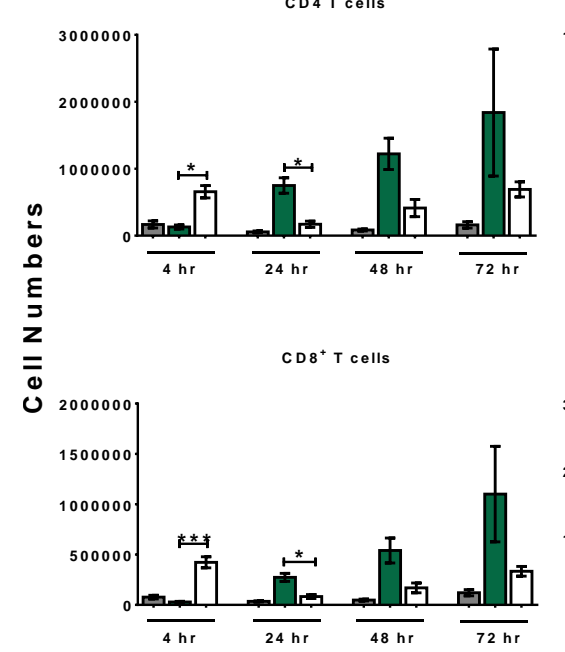

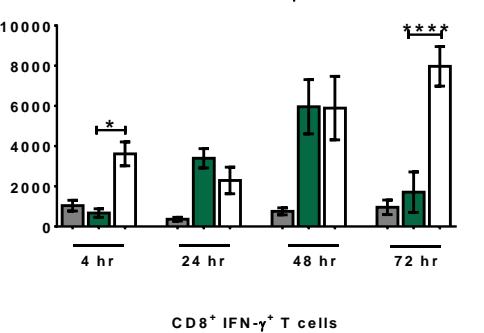

$\operatorname{CD} 8^{+}$IFN-Y - $^{+}$cells

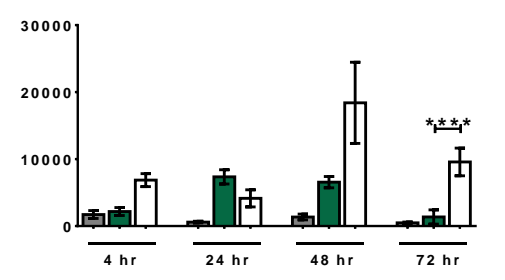

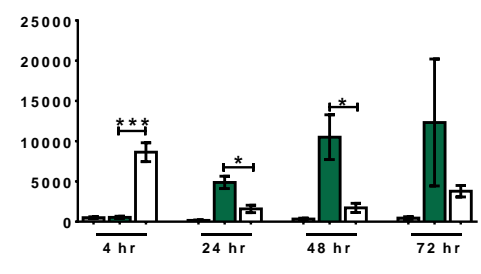

$\operatorname{CD} 8^{+} 1 L 13^{+} T$ cells

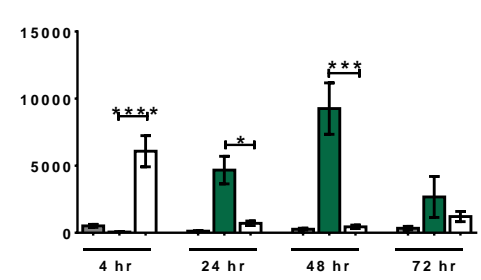

CD $4^{+} \mathrm{IL}-10^{+} \mathrm{T}$ cells

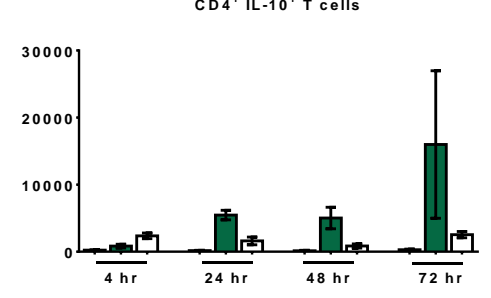

$\mathrm{CDB}^{+} \mathrm{IL}-10^{+} \mathrm{T}$ cells

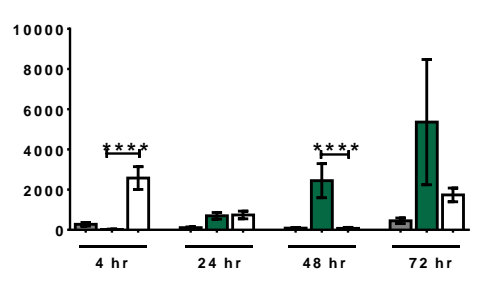

Exposure Groups

Figure 3.9. Intracellular cytokine flow cytometric analysis of the mediastinal lymph nodes. CD4 and CD8 T cells were quantified by multiplying the frequency of each individual cell population by the total cell counts. Data are presented as the average \pm standard error of measure. $N=7-10$ mice/group per time point. $* * * * P \leq 0.0001, * * * P \leq 0.001, * P \leq 0.05$ 


\section{Antibody production}

Western blot analysis using sera from exposed mice was conducted to determine if specific-antibody was produced following repeated nose-only exposures to dry A. fumigatus conidia. The protein profiles from WT and albino conidial extracts were comparable, as shown in Chapter 2. Cross-reactivity between the strains was analyzed to determine if exposures to the albino conidia induced the production of different antibodies compared to WT. No A. fumigatusspecific IgM was produced in either group (data not shown). As shown in Figure 3.10A-C, sera obtained from WT exposed mice had the greatest number of proteins with IgG-specific reactivity compared to the $\Delta a l b l$ sera; however, 8 proteins were recognized by serum IgG in both WT and Aalbl extracts. Prominent bands were identified at approximately $100 \mathrm{kD}, 60 \mathrm{kD}, 52 \mathrm{kD}$, and between 10-15 kD. Interestingly, there was strong reactivity with a doublet band in the albino extract around $40 \mathrm{kD}$ that was prominently recognized by pooled polyclonal sera from each exposure group. The two bands between 10-15 kD were only visible in melanin mutant conidial extracts. Furthermore, we observed a potential increase in the IgG titers between 3-7 days, as bands were darker in the blots incubated with 7D polyclonal sera (Figure 3.10A-C).

Because exposed mice had heightened airway reactivity to methacholine, increased levels of $\mathrm{T}_{\mathrm{H}} 2$ cells in the BALF and $\mathrm{LN}$, and specific-IgG antibody production, it was necessary to determine if $\mathrm{IgE}$ was also produced to further characterize the immune response following repeated exposures. As shown in Figure 3.10D, total $\operatorname{IgE}$ concentrations were significantly increased in each group, despite the presence of melanin. Similar to the western blot data, $\operatorname{IgE}$ titers increased by approximately $1000 \mathrm{ng} / \mathrm{ml}$ (WT exposed) and $1500 \mathrm{ng} / \mathrm{ml}$ ( (albl) between days 3 and 7. Overall, these data indicate that nose-only exposures to dry A. fumigatus conidia result in the production of specific-IgG and total $\mathrm{IgE}$. 
Figure 3.10 Antibody Production
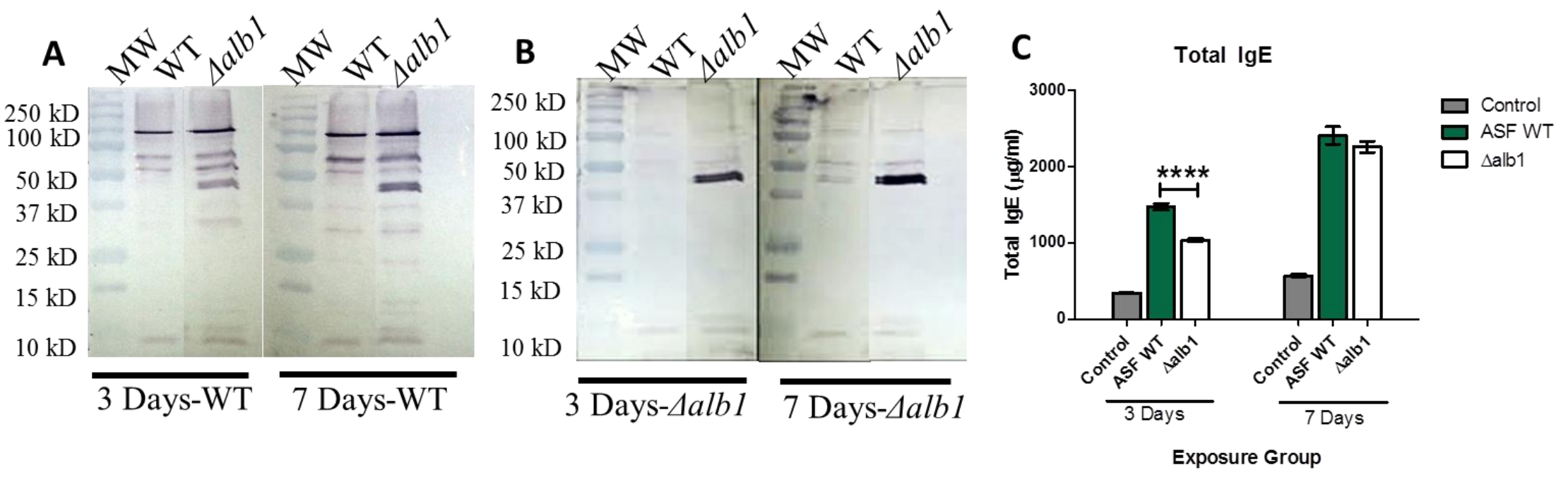

Figure 3.10. IgG and IgE antibody production in wild-type and albino exposed mice. Western blot analysis of serum IgG immunoreactive fungal proteins A) WT conidia, or B) Aalb1 conidia. C) Total IgE quantification via ELISA. Sera from exposed mice was obtained at 3 days or 7 days following the final exposure, and analyzed via a commercially available total IgE ELISA kit. Each sample was prepared in duplicate and the values presented are the average \pm standard error of measure. 


\section{DISCUSSION}

Previously reported data on the effect of fungal exposures on the murine pulmonary immune response has provided valuable insights into the immune mechanisms mediating fungal clearance and pathogenesis. However, these exposure models do not reflect natural human exposures and were often characterized using immunocompromised murine models. In this study, we evaluated the immunocompetent murine immune responses following 8 dry conidial exposures. The concentrations used for exposures are similar to those encountered in contaminated indoor and occupational environments (2). We developed an innovative acoustical generator exposure system to produce homogenous conidial aerosols that could be monitored and adjusted in real-time during exposures. The dry particle generator system used in these experiments was based on the Pitt-3 aerosol generator, which has previously been used to aerosolize cotton dust, low molecular weight particles, and carbon nanotubes (50-52). Experiments comparing aspiration exposures to inhalation exposures of carbon nanotubes reported exacerbation of pulmonary inflammation and fibrosis in mice that were exposed using the acoustical generator. These findings demonstrated the feasibility of using this system for dry fungal exposures (50).

In our system, a nose-only chamber was attached to the acoustical generator to resemble a more natural route of human exposures, limit fungal deposition on the body of the mouse, and thereby remove any impact that ingestion of fungi may have on the immune response. The ability to aerosolize dry conidia with this system also avoided the potential for conidial alterations that likely result following the introduction of conidia to liquid suspensions. Furthermore, rice was also used as a natural substrate for fungal growth. This limited the likelihood that A. fumigatus would produce different immunomodulatory proteins as a result of growth on an artificial, nutrient-rich media. This system also allowed for real-time analysis of the aerosol to ensure the exposure/deposition concentration and size of respirable particles were consistent. These factors have been overlooked in recently published dry A. fumigatus exposure models. Thus, the acoustical generator exposure system is an improved method to reproducibly expose mice to unaltered, dry A. fumigatus conidia for examination of pulmonary immune responses.

In this study, $5 \times 10^{5}$ conidia were used for multiple murine exposures. This concentration of conidia did not induce toxicity, as determined by $>10 \%$ weight loss, yet resulted in the highest amount of lymphocyte proliferation as determined by a modified local lymph node assay. 
Following dry conidial exposures twice weekly for 4 weeks, multiple end points were examined. Histopathology analysis showed that severe inflammation occurred within 4 hours of exposure to WT conidia, which was mediated primarily by macrophages. At the 4 hour time point, conidia were located within macrophages in the surrounding interstitium. Large quantities of mucus in goblet cells were evident, bronchiolar pneumonia was observed, and tissue remodeling/fibrosis processes were evident. By 24 hours, the inflammation was reduced, yet still evident. Large numbers of neutrophils and eosinophils were observed surrounding the larger airways, and giant cell formation was apparent, as were granulomas. Inflammation was mostly resolved by 48 and 72 hours, though fibrotic areas, giant cells, and granulomas were still observed. With conidia, many had begun to swell by 4 hours, but few germ tubes were evident. By 24-48 hours, many conidia had begun to form longer hyphae; however, by 72 hours, most conidia were cleared. These results are consistent with previously published data from other immunocompetent animal models of fungal exposures $(8,16,34,53)$. Using melanin mutant strains, these data demonstrated that inflammation was not dependent on the extent of melanization, but changes in the clearance of conidia were impacted by the melanin content. These findings are in agreement with the data presented in Chapter 2.

Importantly, fewer albino conidia were observed in the histopathology analysis at the earlier time points, despite being exposed to equal concentrations. Decreased albino conidia at 5 hours post aspiration exposure were also reported in Chapter 2. Recent studies have shown that epithelial cells lining the respiratory tract contain toll-like receptors (TLRs), including TLR-2 and TLR-4 that specifically recognize fungal conidia and hyphae, and can result in the translocation of transcription factor, NF- $\kappa \mathrm{B}$ and the production of proinflammatory cytokines $(54,55)$. The results of these studies suggest that these cells are able to efficiently phagocytose and degrade the albino conidia in the upper airways, thereby affecting the deposition of conidia within the lungs. Furthermore, it has been hypothesized that the lack of a melanin layer enhances the accessibility of important PAMPs, such as $\beta$-glucans, that directly impact innate-mediated clearance and production of proinflammatory responses through PRRs such as Dectin-1, though this has not yet been determined (45). Interactions of accessible PAMPs with innate PRRs would greatly influence the rate of clearance, and could offer a potential explanation for the lower concentration of albino conidia present at 4 hours post-exposure $(12,54,56,57)$. The lack of melanin in the albino conidia may also result in increased susceptibility to innate clearance 
mechanisms $(35,41,43)$. Thus, it is also likely that these conidia are cleared at a much faster rate than WT conidia. This finding may also account for the slightly decreased levels of conidia observed at 5 hours post aspiration exposure presented in Chapter 2.

The presence of melanin impacted the recruitment kinetics of immune cells in BALF and mediastinal lymph nodes, as total cell numbers peaked at 4 hours in mice exposed to albino conidia, but not until 48 hours in WT exposed mice. By 72 hours, cell numbers had decreased to slightly above control animals, which was similar to the observed clearance of most conidia by this time. These results show that there is a delayed response to melanized conidia, is likely related to the ability of melanin to mask and neutralize many innate immune functions. Interestingly, a downward shift in alveolar macrophage populations was observed in the flow cytometry dot plots of exposed mice. This shift was more evident in mice exposed to WT conidia. Alternatively activated macrophages (M2) are present during allergic responses, and play a role in tissue repair. Fei et al. have reported that M2 cells are the predominant macrophage cell type present, and are induced shortly following exposure to A. fumigatus conidia (8). Given the downward shift from the population in control mice, it is possible that this population consists of alternatively activated macrophages (M2), though M2-specific surface markers were not examined (8).

Intracellular cytokine analysis of BALF components provided greater insight into the pulmonary immune responses following repeated dry conidia exposures. The data demonstrated that CD4 and CD8 T cell numbers may be dependent on melanin, as exposures to WT conidia resulted in greater numbers of these cell types. Higher levels of CD4 T cell populations were observed at 4 and 48 hours post exposure to WT conidia. It is possible that these CD4 T cell populations were the result of innate recognition of resting conidia. As conidia began to germinate, a secondary CD4 $\mathrm{T}$ cell response appeared to occur, potentially as a result of the inflammation associated with recognition and clearance of the swollen conidia that occurred between 24 and 48 hours post exposure. Further, peak CD4 IL-17 ( $\left.\mathrm{T}_{\mathrm{H}} 17\right)$ production occurred at 48 hours in mice exposed to WT conidia, which corresponded to the peak neutrophil numbers in the BALF. Previous reports have indicated that a major role for $\mathrm{T}_{\mathrm{H}} 17$ cells includes the recruitment of neutrophils, which highlights an important host defense mechanism for induction of $\mathrm{T}_{\mathrm{H}} 17$ cells in the clearance of fungi. Additionally, there was also concomitant $\mathrm{T}_{\mathrm{H}} 1$ and $\mathrm{T}_{\mathrm{H}} 2$ responses over the time course of the study, which has been previously been shown in other $A$. 
fumigatus aspiration models. This may have a functional role in the induction of allergy, as over time, the $\mathrm{T}_{\mathrm{H}} 2$ responses can outweigh the inflammatory reactions to limit tissue injury.

CD4 $\mathrm{T}$ cell responses within the lymph nodes of mice exposed to WT conidia increased over time, likely indicating the continual antigen presentation, lymphocyte activation, and clonal expansion at this site $(58,59)$. Levels of CD4 IFN- $\gamma\left(\mathrm{T}_{\mathrm{H}} 1\right)$, IL-13 and IL-9 (allergy associated $\mathrm{T}_{\mathrm{H}} 2$ ), and IL-17 and IL-10 (immunoregulatory cytokines) peaked at 72 hours in mice exposed to WT conidia. These data agree with the BALF data, and indicate concurrent $T_{H} 1$ and $T_{H} 2$ responses. There were minimal differences in these cell numbers in mice exposed to albino conidia, with the exception of IFN- $\gamma$ production. Because melanin is known to dampen proinflammatory responses, this result was expected in the BALF (45). However, LN responses to albino conidia have not been previously examined. Through murine exposure to Cryptococcus neoformans strains with different levels of melanization, Huffnagle et al. observed a marked decreased in lymphocyte expansion in the mediastinal lymph nodes and serum antibody responses in animals that were exposed the high melanin fungus (60). The authors further hypothesized that these results are likely related to the decreased proinflammatory response that occurs following exposure to the highly melanized fungus. Taken together with the data presented in these studies, A. fumigatus melanin may also dampen adaptive immune responses by limiting the induction of proinflammatory lymphocytes. Alternatively, because there are fewer albino conidia present at the earliest time point examined, it is also possible that the low level of these conidia is below a threshold that induces lymphocyte proliferation. Further modified local lymph node assays to determine lymphocyte proliferation following exposures to albino conidia are required to clarify this observation.

There is minimal information available concerning the role of CD8 $\mathrm{T}$ cell responses in $A$. fumigatus induced immune responses, although studies have shown that germination can impact the production of memory CD8 $\mathrm{T}$ cell responses and fungal clearance. In these studies, total BALF CD8 T cells increased by 24 hours and then steadily declined over time. CD8 IFN- $\gamma$ and IL-13 production was highest at 4 hours. IL-10 production increased as IFN- $\gamma$ levels declined, which is consistent with the role of IL-10 to limit the inflammatory response. Importantly, Tc17 cells mirrored the total CD8 $\mathrm{T}$ cell numbers, which peaked at 24 hours. These cells were the predominant CD8 T cells, comprising approximately $1 / 3$ of the parent population. 
The importance of Tc17 cells in immune responses to A. fumigatus conidia has not been previously shown. As discussed in Chapter 2, Tc17 cells are a unique subset of $\mathrm{CD}^{+} \mathrm{T}$ cells that have recently been associated with viral immunity (viral clearance), pulmonary inflammatory responses, patients with systemic lupus erythematosus, control of tumor growth, and contact dermatitis. Tc17 cells demonstrate functional plasticity, and are reported to produce proinflammatory cytokines and chemokines responsible for the enhanced recruitment of macrophages, natural killer cells, and neutrophils. Aside from the studies presented in this dissertation, these cells have not been previously identified in models of A. fumigatus exposures, and may indicate a novel role for Tc17 cells in the immune responses to the fungus. Within 24 hours of peak Tc17 responses, the maximum numbers of macrophage, neutrophils, and eosinophils were present in the BALF. These results are consistent with the reported role for Tc17 cells innate cell recruitment. The response was also fungal specific, as exposures to silicone beads of the same size as conidia did not induce Tc17 cell responses. Most importantly, the increase in Tc17 cells appeared to correlate with an increase in the germination of WT conidia. These results demonstrate that Tc17 cells are likely activated and recruited to the lungs of mice upon swelling and germination of conidia.

LN CD8 T cells also increased over time in mice exposed to WT conidia, while mice levels were highest at 4 hours, followed by a rapid decrease at 24 hours, and a steady incline over time in mice exposed to albino conidia. CD8 IFN- $\gamma$ production was limited in WT exposed mice, though levels peaked at 24 hours followed by a steady decline. Albino conidial exposures resulted in higher levels of CD8 IFN- $\gamma$ than in WT, correlating to data obtained from the BALF. Peak responses in CD8 IL-13, IL-9, and IL-10 occurred at 4 hours in mice exposed to albino conidia; however, levels dropped off and then steadily increased to 72 hours. The heightened levels at 4 hours may be due to a faster rate of albino conidia clearance, which may potentially increase the rate of antigen presenting cell trafficking to LN. This may also be compounded by the heighted inflammatory responses occurring following exposures to albino conidia.

Previous data has shown limited antibody responses following repeated aspiration exposures; however, it remained unknown if repeated dry exposures would impact antibody production. Therefore, we examined mediastinal lymph node B cell cellularity following dry exposures. As was indicated in BALF analysis, albino conidia induced the greatest number of B cells at 4 hours, followed by a sudden decrease at 24 hours, which followed an upward trend by 
72 hours. However, LN B cell numbers were highest at the latest time point examined (72 hours) post-exposure in WT exposed mice at 72 hours post-exposure. This dual-phase response to albino conidia is consistent with 'classical' activation of the immune response, with the innate effector phase occurring immediately following exposure, and the adaptive phase starting approximately 72 hours after re-exposure to the same antigen. As the albino conidia were cleared, the overall immune response decreased until any conidia remaining were processed and presented within the LN. However, this difference in cellular recruitment at each time point in response to albino conidia did not impact the production of antibody. This is likely because LN B cell numbers were comparable between the exposure groups at this time point and strongly correlated to helper $\mathrm{T}$ cell responses, which are required for activation and expansion of $\mathrm{B}$ cell responses.

Since total $\operatorname{IgE}+$ B cells were consistently high in mice exposed to WT conidia, specific antibody responses, including IgG and IgM reactivity, was analyzed using western blotting. Initial experiments indicated that there was no detectable specific-IgM production; however, several IgG-reactive proteins were visualized using polyclonal pooled sera from mice sacrificed 3 and 7 days following the final exposure. Sera from each group were also cross-reactive against conidial extracts from each fungal strain, regardless of melanization. Interestingly, WT sera reacted strongly with a greater number of bands in the albino extract than in WT or Aarp2 extracts. These results suggest that additional A. fumigatus-specific antibodies were produced that may not have been previously identified, as cross-reactivity against albino conidia has not been examined prior to these studies. It is possible that the additional reactive proteins are tightly cross-linked to the melanin within the conidial wall, and thus, are diminished in the WT conidial extract. Further, the reactive proteins may be more soluble in the albino conidia, and therefore are present in higher concentrations in the albino extract, resulting in stronger band intensity following western blot analysis. Furthermore, the intensity of the bands were increased in blots incubated with sera obtained from mice 7 days post-final exposure. Since antibody production accumulates overtime, these results are consistent with the knowledge that maximal adaptive immunity and antibody production commonly occurs between 7-10 days after re-exposure (61). These results are significant because some allergic conditions caused by frequent fungal inhalation are mediated by both IgG and IgE. 
Total IgE was analyzed in pooled sera obtained from exposed mice at 3 and 7 days postfinal exposure. There was a marked increase in the total $\operatorname{IgE}$ present in sera from mice exposed to both WT and albino conidia, and this level also increased between days 3-7. Importantly, the total IgE levels presented increased nearly 100 -fold over those presented by Schuh and colleagues, though this is likely a function of exposure duration, as the same strain of $A$. fumigatus WT were used in both studies (34). Taken together, these results indicate that melanin does not significantly impact the production of fungal-specific antibodies, nor does it impact the induction of allergy in our model. Repeated nose-only exposures to dry conidia, despite melaninization, appear to induce allergic phenotypes. Further experiments to identify of the specific components that mediate these responses are required for better understanding of the fungal allergy induction process.

In conclusion, we have developed a novel, sophisticated dry fungal aerosol exposure system that can be used to characterize the immune responses against environmentally and occupationally relevant fungal bioaerosol sources. This is significant because it allows for experiments to be completed that more closely model natural human exposures than those models that are currently reported in the peer reviewed literature. Furthermore, this exposure model also accounts for the potential impact of other cells, which may aid in shaping the developing immune response, and were overlooked using aspiration and intratracheal instillation models. We have shown that the kinetics of cellular inflammatory response is affected by the presence of melanin. Most importantly, results from this study suggest that Tc17 cells recognize and may respond to A. fumigatus germination, and are likely the major source of IL-17 responsible for heightened recruitment of innate phagocytes. All presented results were specific to fungi, as exposure to control silicone beads induced innate inflammation which was rapidly resolved, and did not result in an increase in T cells in the BALF or LN (data not shown). Additionally, we observed the production of specific $\operatorname{IgG}$ to numerous immuno-dominant proteins. Identification of these biomarker antigens may be useful in improving diagnostics through identifying potential biomarkers of exposures/infection/allergy, and in developing improved treatment options or potential A. fumigatus vaccines. 


\section{REFERENCES}

1. Latgé, J.-P., and W. J. Steinbach. 2009. Aspergillus fumigatus And Aspergillosis. ASM Press.

2. Eduard, W. 2009. Fungal spores: a critical review of the toxicological and epidemiological evidence as a basis for occupational exposure limit setting. Critical Reviews in Toxicology 39: 799-864.

3. Latgé, J. P. 1999. Aspergillus fumigatus and aspergillosis. Clinical Microbiology Reviews 12: 310-350.

4. Devereux, G. 2006. The increase in the prevalence of asthma and allergy: food for thought. Nat Rev Immunol 6: 869-874.

5. Simon-Nobbe, B., U. Denk, V. Pöll, R. Rid, and M. Breitenbach. 2008. The spectrum of fungal allergy. International Archives of Allergy and Immunology 145: 58-86.

6. Templeton, S. P., A. D. Buskirk, B. J. Green, D. H. Beezhold, and D. Schmechel. 2010. Murine models of airway fungal exposure and allergic sensitization. Medical Mycology 48: 217-228.

7. Aimanianda, V., J. Bayry, S. Bozza, O. Kniemeyer, K. Perruccio, S. R. Elluru, C. Clavaud, S. Paris, A. A. Brakhage, S. V. Kaveri, L. Romani, and J.-P. Latge. 2009. Surface hydrophobin prevents immune recognition of airborne fungal spores. Nature 460: 1117-1121.

8. Bhatia, S., M. Fei, M. Yarlagadda, Z. Qi, S. Akira, S. Saijo, Y. Iwakura, N. van Rooijen, G. Gibson, C. St Croix, A. Ray, and P. Ray. 2011. Rapid host defense against Aspergillus fumigatus involves alveolar macrophages with a predominance of alternatively activated phenotype. PLoS ONE 6: e15943-e15943.

9. Bozza, S., R. Gaziano, A. Spreca, A. Bacci, C. Montagnoli, P. di Francesco, and L. Romani. 2002. Dendritic cells transport conidia and hyphae of Aspergillus fumigatus from the airways to the draining lymph nodes and initiate disparate Th responses to the fungus. The Journal of Immunology 168: 1362-1371.

10. Capilla, J., K. V. Clemons, and D. A. Stevens. 2007. Animal models: an important tool in mycology. Medical Mycology 45: 657-684.

11. Denis, O., S. van den Brûle, J. Heymans, X. Havaux, C. Rochard, F. Huaux, and K. Huygen. 2007. Chronic intranasal administration of mould spores or extracts to unsensitized mice leads to lung allergic inflammation, hyper-reactivity and remodelling. Immunology 122: 268-278.

12. Dubourdeau, M., R. Athman, V. Balloy, M. Huerre, M. Chignard, D. Philpott, J.-P. Latg, and O. Ibrahim-Granet. 2006. Aspergillus fumigatus induces innate immune responses in alveolar macrophages through the MAPK pathway independently of TLR2 and TLR4. The Journal of Immunology 177: 3994-4001.

13. Erpenbeck, V. J., M. Ziegert, D. Cavalet-Blanco, C. Martin, R. Baelder, T. Glaab, A. Braun, W. Steinhilber, B. Luettig, S. Uhlig, H. G. Hoymann, N. Krug, and J. M. Hohlfeld. 2006. Surfactant protein D inhibits early airway response in Aspergillus fumigatus-sensitized mice. Clinical and Experimental Allergy 36: 930-940.

14. Grunig, G., and V. P. Kurup. 2003. Animal models of allergic bronchopulmonary aspergillosis. Frontiers in Bioscience 8: e157-171.

15. Hellings, P. W., A. Kasran, Z. Liu, P. Vandekerckhove, A. Wuyts, L. Overbergh, C. Mathieu, and J. L. Ceuppens. 2003. Interleukin-17 orchestrates the granulocyte influx 
into airways after allergen inhalation in a mouse model of allergic asthma. American Journal of Respiratory Cell and Molecular Biology 28: 42-50.

16. Hogaboam, C. M., K. Blease, B. Mehrad, M. L. Steinhauser, T. J. Standiford, S. L. Kunkel, and N. W. Lukacs. 2000. Chronic airway hyperreactivity, goblet cell hyperplasia, and peribronchial fibrosis during allergic airway disease induced by Aspergillus fumigatus. The American Journal of Pathology 156: 723-732.

17. Kurup, V. P. 1984. Interaction of Aspergillus fumigatus spores and pulmonary alveolar macrophages of rabbits. Immunobiology 166: 53-61.

18. Murdock, B. J., N. R. Falkowski, A. B. Shreiner, A. A. Sadighi Akha, R. A. McDonald, E. S. White, G. B. Toews, and G. B. Huffnagle. 2012. Interleukin-17 Drives Pulmonary Eosinophilia following Repeated Exposure to Aspergillus fumigatus Conidia. Infection and immunity 80: 1424-1436.

19. Murdock, B. J., A. B. Shreiner, R. A. McDonald, J. J. Osterholzer, E. S. White, G. B. Toews, and G. B. Huffnagle. 2011. Coevolution of $\mathrm{T}_{\mathrm{H}} 1, \mathrm{~T}_{\mathrm{H}} 2$, and $\mathrm{T}_{\mathrm{H}} 17$ responses during repeated pulmonary exposure to Aspergillus fumigatus conidia. Infect. Immun. 79: 125135.

20. Rivera, A., T. Hohl, N. Collins, I. Leiner, A. Gallegos, S. Saijo, J. Coward, Y. Iwakura, and E. Pamer. 2011. Dectin-1 diversifies Aspergillus fumigatus-specific T cell responses by inhibiting $\mathrm{T}$ helper type $1 \mathrm{CD} 4 \mathrm{~T}$ cell differentiation. The Journal of Experimental Medicine 208: 369-381.

21. Rivera, A., H. L. Van Epps, T. M. Hohl, G. Rizzuto, and E. G. Pamer. 2005. Distinct CD4+T-cell responses to live and heat-inactivated Aspergillus fumigatus conidia. Infection and Immunity 73: 7170-7179.

22. Thurston, J. R., S. J. Cysewski, and J. L. Richard. 1979. Exposure of rabbits to spores of Aspergillus fumigatus or Penicillium sp: survival of fungi and microscopic changes in the respiratory and gastrointestinal tracts. American Journal of Veterinary Research 40: 1443-1449.

23. Nanguy, S. P.-M., J.-M. Perrier-Cornet, M. Bensoussan, and P. Dantigny. 2010. Impact of water activity of diverse media on spore germination of Aspergillus and Penicillium species. International Journal of Food Microbiology 142: 273-276.

24. Green, B. J., E. R. Tovey, J. K. Sercombe, F. M. Blachere, D. H. Beezhold, and D. Schmechel. 2006. Airborne fungal fragments and allergenicity. Medical Mycology 44 Suppl 1: S245-255.

25. Sporik, R. B., L. K. Arruda, J. Woodfolk, M. D. Chapman, and T. A. Platts-Mills. 1993. Environmental exposure to Aspergillus fumigatus allergen (Asp f I). Clinical and Experimental Allergy 23: 326-331.

26. Coméra, C., K. André, J. Laffitte, X. Collet, P. Galtier, and I. Maridonneau-Parini. 2007. Gliotoxin from Aspergillus fumigatus affects phagocytosis and the organization of the actin cytoskeleton by distinct signalling pathways in human neutrophils. Microbes and Infection 9: 47-54.

27. Curtis, L., A. Lieberman, M. Stark, W. Rea, and M. Vetter. 2004. Adverse health effects of indoor molds. Journal of Nutritional \& Environmental Medicine 14: 261-274.

28. Cusumano, V., G. B. Costa, and S. Seminara. 1990. Effect of aflatoxins on rat peritoneal macrophages. Applied and Environmental Microbiology 56: 3482-3484. 
29. Eichner, R. D., M. Al Salami, P. R. Wood, and A. Müllbacher. 1986. The effect of gliotoxin upon macrophage function. International Journal of Immunopharmacology 8: 789-797.

30. Kamei, K., and A. Watanabe. 2005. Aspergillus mycotoxins and their effect on the host. Medical Mycology 43 Suppl 1: S95-99.

31. Pahl, H. L., B. Krauss, K. Schulze-Osthoff, T. Decker, E. B. Traenckner, M. Vogt, C. Myers, T. Parks, P. Warring, A. Mühlbacher, A. P. Czernilofsky, and P. A. Baeuerle. 1996. The immunosuppressive fungal metabolite gliotoxin specifically inhibits transcription factor NF-kappaB. The Journal of Experimental Medicine 183: 1829-1840.

32. Mitchell, C. G., J. Slight, and K. Donaldson. 1997. Diffusible component from the spore surface of the fungus Aspergillus fumigatus which inhibits the macrophage oxidative burst is distinct from gliotoxin and other hyphal toxins. Thorax 52: 796-801.

33. Hoselton, S. A., A. E. Samarasinghe, J. M. Seydel, and J. M. Schuh. 2010. An inhalation model of airway allergic response to inhalation of environmental Aspergillus fumigatus conidia in sensitized BALB/c mice. Medical Mycology 48: 1056-1065.

34. Samarasinghe, A. E., S. A. Hoselton, and J. M. Schuh. 2011. A comparison between intratracheal and inhalation delivery of Aspergillus fumigatus conidia in the development of fungal allergic asthma in C57BL/6 mice. Fungal Biology 115: 21-29.

35. Jacobson, E. S. 2000. Pathogenic roles for fungal melanins. Clinical Microbiology Reviews 13: 708-717.

36. Wheeler, M. H., and A. A. Bell. 1988. Melanins and their importance in pathogenic fungi. Current Topics in Medical Mycology 2: 338-387.

37. Langfelder, K., B. Jahn, H. Gehringer, A. Schmidt, G. Wanner, and A. A. Brakhage. 1998. Identification of a polyketide synthase gene (pksP) of Aspergillus fumigatus involved in conidial pigment biosynthesis and virulence. Medical Microbiology and Immunology 187: 79-89.

38. Tsai, H. F., Y. C. Chang, R. G. Washburn, M. H. Wheeler, and K. J. Kwon Chung. 1998. The developmentally regulated alb1 gene of Aspergillus fumigatus: its role in modulation of conidial morphology and virulence. Journal of bacteriology 180: 3031-3038.

39. Tsai, H. F., R. G. Washburn, Y. C. Chang, and K. J. Kwon Chung. 1997. Aspergillus fumigatus arp1 modulates conidial pigmentation and complement deposition. Molecular microbiology 26: 175-183.

40. Brakhage, A. A., K. Langfelder, G. Wanner, A. Schmidt, and B. Jahn. 1999. Pigment biosynthesis and virulence. Contributions to Microbiology 2: 205-215.

41. Butler, M. J., A. W. Day, J. M. Henson, and N. P. Money. 2001. Pathogenic Properties of Fungal Melanins. Mycologia 93: 1-8.

42. Jahn, B., F. Boukhallouk, J. Lotz, K. Langfelder, G. Wanner, and A. A. Brakhage. 2000. Interaction of human phagocytes with pigmentless Aspergillus conidia. Infection and Immunity 68: 3736-3739.

43. Thywiben, A., T. Heinekamp, H.-M. Dahse, J. Schmaler-Ripcke, S. Nietsche, P. F. Zipfel, and A. A. Brakhage. 2011. Conidial dihydroxynaphthalene melanin of the human pathogenic fungus Aspergillus fumigatus interferes with the host endocytosis pathway. Frontiers in Microbiology 2.

44. Volling, K., A. A. Brakhage, and H. P. Saluz. 2007. Apoptosis inhibition of alveolar macrophages upon interaction with conidia of Aspergillus fumigatus. FEMS Microbiology Letters 275: 250-254. 
45. Chai, L. Y. A., M. G. Netea, J. Sugui, A. G. Vonk, W. W. J. van de Sande, A. Warris, K. J. Kwon-Chung, and B. Jan Kullberg. 2010. Aspergillus fumigatus conidial melanin modulates host cytokine response. Immunobiology 215: 915-920.

46. Jarvis, B. B., W. G. Sorenson, E.-L. Hintikka, M. Nikulin, Y. Zhou, J. Jiang, S. Wang, S. Hinkley, R. A. Etzel, and D. Dearborn. 1998. Study of toxin production by isolates of Stachybotrys chartarum and Memnoniella echinata isolated during a study of pulmonary hemosiderosis in infants. Applied and Environmental Microbiology 64: 3620-3625.

47. Raabe, O. G., M. A. Al-Bayati, S. V. Teague, and A. Rasolt. 1988. Regional deposition of inhaled monodisperse coarse and fine aerosol particles in small laboratory animals. Annals of Occupational Hygiene 32: 53-63.

48. Stevens, W. W., T. S. Kim, L. M. Pujanauski, X. Hao, and T. J. Braciale. 2007. Detection and quantitation of eosinophils in the murine respiratory tract by flow cytometry. $J$ Immunol Methods 327: 63-74.

49. Foster, B., C. Prussin, F. Liu, J. K. Whitmire, and J. L. Whitton. 2007. Detection of intracellular cytokines by flow cytometry. In Curr Protoc Immunol. John Wiley and Sons, Inc.

50. Shvedova, A. A., E. Kisin, A. R. Murray, V. J. Johnson, O. Gorelik, S. Arepalli, A. F. Hubbs, R. R. Mercer, P. Keohavong, N. Sussman, J. Jin, J. Yin, S. Stone, B. T. Chen, G. Deye, A. Maynard, V. Castranova, P. A. Baron, and V. E. Kagan. 2008. Inhalation vs. aspiration of single-walled carbon nanotubes in C57BL/6 mice: inflammation, fibrosis, oxidative stress, and mutagenesis. American Journal of Physiology - Lung Cellular and Molecular Physiology 295: L552-L565.

51. Weyel, D. A., and M. Corn. Characteristics of tantalum dust and dust generator for bronchography. Invest Radiol 10: 500-510.

52. Rosato, L. M., D. A. Weyel, and Y. C. Alarie. 1988. A low airflow aerosol generator for the delivery of respirable aerosols. Journal of Aerosol Medicine 1: 127-132.

53. Templeton, S. P., A. D. Buskirk, B. Law, B. J. Green, and D. H. Beezhold. 2011. Role of germination in murine airway CD8 T-cell responses to Aspergillus conidia. PLoS ONE 6: e18777.

54. Meier, A., C. J. Kirschning, T. Nikolaus, H. Wagner, J. Heesemann, and F. Ebel. 2003. Toll-like receptor (TLR) 2 and TLR4 are essential for Aspergillus-induced activation of murine macrophages. Cellular Microbiology 5: 561-570.

55. Mambula, S. S., K. Sau, P. Henneke, D. T. Golenbock, and S. M. Levitz. 2002. Toll-like receptor (TLR) signaling in response to Aspergillus fumigatus. The Journal of Biological Chemistry 277: 39320-39326.

56. Brown, G. D. 2006. Dectin-1: a signalling non-TLR pattern-recognition receptor. Nature Reviews. Immunology 6: 33-43.

57. Werner, J. L., A. E. Metz, D. Horn, T. R. Schoeb, M. M. Hewitt, L. M. Schwiebert, I. Faro-Trindade, G. D. Brown, and C. Steele. 2009. Requisite role for the dectin-1 $\beta$-glucan receptor in pulmonary defense against Aspergillus fumigatus. The Journal of Immunology 182: 4938-4946.

58. Lambrecht, B. N., and H. Hammad. 2003. Taking our breath away: dendritic cells in the pathogenesis of asthma. Nat Rev Immunol 3: 994-1003.

59. Obst, R., H.-M. van Santen, D. Mathis, and C. Benoist. 2005. Antigen persistence is required throughout the expansion phase of a $\mathrm{CD} 4+\mathrm{T}$ cell response. The Journal of Experimental Medicine 201: 1555-1565. 
60. Huffnagle, G., G. Chen, J. Curtis, R. McDonald, R. Strieter, and G. Toews. 1995. Downregulation of the afferent phase of $\mathrm{T}$ cell-mediated pulmonary inflammation and immunity by a high melanin-producing strain of Cryptococcus neoformans. The Journal of Immunology 155: 3507-3516.

61. Murphy, K., P. Travers, M. Walport, and C. Janeway. 2012. Janeway's immunobiology. Garland Science, New York. 
Chapter 4

Discussion 


\section{DISCUSSION}

Aspergillus fumigatus is a leading cause of invasive disease in immunocompromised individuals (1-3). Although A. fumigatus has predominantly been examined in this context, it is also responsible for inducing a large number of diseases characterized by persistent colonization and the induction/exacerbation of allergy and asthma phenotypes. These adverse health outcomes impact a greater population of individuals than invasive disease, and the associated health care costs are a major public health burden (4-10). Despite the large population of individuals affected by fungal-mediated allergies and asthma, the immunological mechanisms of disease induction following repeated fungal exposures in immunocompetent individuals have remained largely uncharacterized. To address this knowledge gap in the peer reviewed literature, the primary goal of this dissertation was to characterize the immune response(s) following subchronic A. fumigatus exposures in an immunocompetent murine model.

Numerous manuscripts have been published that discuss the impact of A. fumigatus exposure on the immune system and these studies have led to the current understanding in the field $(11,12)$. However, many reports only present data derived from in vitro cell culture systems (13-26). Those studies that examine the impact of exposure in in vivo systems use animal models but these often do not resemble natural human exposures. Further, these reports mainly contain data obtained from single exposures in immunocompromised mice and utilize protocols that require exposure to either fungal extracts or suspensions of fungal particles. In natural environments, humans are exposed to approximately $10^{4}$ conidia $/ \mathrm{m}^{3}$ daily, although the concentration may vary greatly based on the geographical location, season, and disturbance to colonized surfaces (27). Fungal bioaerosols consist of fungal conidia, hyphae or particulates that have been released from environmental substrates. Resting A. fumigatus conidia are $3 \mathrm{um}$ in diameter and contain numerous antigenic molecules, which are likely impacted by the growth substrate and the metabolic activity of the organism. Upon interaction with liquid, these antigens are potentially solubilized or diluted, resulting in the removal of natural PAMPs that may influence host-mediated immune recognition. Further, the activation of metabolism in resting conidia can be rapid upon interaction with aqueous buffers (28). Upon germination, numerous changes occur in the conidial surface structure, accessibility of immunostimulatory proteins, and production of immunosuppressive toxins (3). For example, within 3 hours of inoculation in Hank's Balanced Salt Solution, a conidia-specific toxin diffuses from A. fumigatus conidia and 
inhibits innate immune responses (29). Therefore, the secondary aim of this dissertation was to develop an exposure system that accounted for these limiting variables and more closely resembled personal exposures to dry fungal particles obtained from natural substrates.

Repeated exposures to elevated concentrations of fungi leading to antigen persistence are hypothesized to be a major factor responsible for allergic sensitization and asthma (7). However, aside from host genetic predisposition and the production of antigens during fungal germination, mechanisms of allergy and asthma induction following exposure remain largely unknown (6, 8, 9, 30). Fungal melanin, a known A. fumigatus virulence factor, has been shown to protect conidia from innate recognition and clearance $(31,32)$. Experiments with albino conidia, derived from a clinical isolate of A. fumigatus, have shown a decrease in virulence when compared to WT conidia $(33,34)$. WT conidia have also been shown to bind fewer complement C3 molecules than albino isolates, leading to decreased opsonization of the fungus (35). Complement regulatory molecules, such as Factor $\mathrm{H}$ which inhibits complement binding, also bind to WT conidia in a melanin-dependent manner (36). This further protects the conidia from complementmediated phagocytosis and membrane attack complex-induced lysis. Melanized conidia are also able to quench ROS produced by macrophages and neutrophils, the first phagocytes to respond to A. fumigatus following inhalation $(32,37-40)$. Additionally, the presence or absence of melanin does not affect phagocytosis $(34,37)$. However, the melanin in WT conidia has been shown to inhibit host cell apoptosis, as well as acidification of the phagolysosome $(41,42)$. Overall, the presence of melanin in A. fumigatus conidia has been shown to protect the fungus from innate clearance, leading to persistence within the lungs, which may impact allergy and asthma induction. Therefore, the third aim of this dissertation was to examine the influence of melanin on the induction of allergy/asthma in a murine model of repeated exposures to WT and melanin-deficient A. fumigatus conidia.

The influence of fungal melanin on the immunocompetent murine immune responses following multiple aspiration or dry A. fumigatus conidia exposures was examined in Chapters 2 and 3 of this dissertation. Chapter 2 characterized the immune responses following 5 aspirations (Figure 2.3A) of A. fumigatus wild-type or melanin-deficient strains. This method was used because of historical data which showed reproducible and efficient pulmonary antigen delivery. Mice were sacrificed at a single time-point, 72 hours after the final aspiration exposure, and multiple end points were examined. For Chapter 3, a sophisticated, nose-only acoustical 
generator exposure system capable of producing dry, natural A. fumigatus conidial aerosols was developed. Mice were exposed twice weekly for 4 weeks to WT or the melanin-deficient strains. Thus, data obtained from this murine model of repeated exposures more accurately reflects the response that occurs following natural exposures. Mice were sacrificed at 4, 24, 48, and 72 hours after the $8^{\text {th }}$ exposure to examine the time-dependent influx of different cells to the airways and mediastinal lymph nodes.

Ideally, exposure schedules would have been the same between the two sets of experiments; however, the low levels of allergy phenotypes and the lack of detectable serum antibody production in mice exposed via aspiration made it difficult to assess mechanisms of allergic induction. As a result of the observations in Chapter 2, the exposure schedule was lengthened in an effort to enhance allergic responses. Furthermore, the exposure concentration was decreased to $5 \times 10^{6}$ conidia (estimated lower airway deposition concentration) in the acoustical generator studies due to higher toxicity associated with $1 \times 10^{6}$ conidia. Despite these differences in exposure concentrations between the two studies, the histopathology results and cellularity were comparable (Appendix Table A2). These results were also similar to those published by other laboratories that used varying concentrations in murine exposure models.

In spite of the variations in exposure schedules and concentrations between Chapter 2 and 3, several differences were observed (Appendix Table A2). Total BALF counts were similar between exposure models, although there was an apparent decrease in neutrophil and eosinophil populations in mice exposed to albino conidia via acoustical generation (Appendix Table A2). Another marked difference was the number of remaining albino conidia in exposed lungs. Mice exposed via aspiration had greater concentrations of conidia remaining at 5 hours after exposure than did the same group exposed via the acoustical generator at 4 hours, even when the difference in exposure concentrations was taken into account (Appendix Table A2). The difference in neutrophil and eosinophil numbers may be due to the lower number of albino conidia present within the lung at 4 hours following acoustical generator exposures than were observed in albino aspiration exposures. The nose-only dry aerosol exposure chamber accounted for the influence that other host-fungal interactions may have had on the overall immune response to fungi. The respiratory tract is lined with specialized epithelial cells which have recently been shown to specifically recognize, engulf, and degrade A. fumigatus conidia (16, 25, 43, 44). Conidial germination within these cells is a mechanism used by the fungus during 
invasive infection $(3,44)$. Furthermore, A. fumigatus produces proteases and antigens that inhibit the ciliary beat frequency of the airway epithelial cells, thereby limiting their removal through the mucocilliary escalator. These proteases are thought to break down the tight junctions between the epithelial cells, providing a path for invasion into the underlying tissues $(24,45)$. The interaction between epithelial cells and A. fumigatus conidia is mediated through PRRs, including TLR-2 and TLR-4, which results in the production of proinflammatory cytokines (4649). These cytokines aid in the recruitment of professional phagocytes to the affected site, thereby increasing the rate of clearance (3).

Multiple studies described the presence of inflammasomes within epithelial cells $(50,51)$. Inflammasomes are composed of several cytoplasmic proteins that, under specific signaling events including activation of intracellular PRRs, bind together to form complexes. These complexes form a signaling pathway that results in the induction or inhibition of different transcription factors, most commonly nuclear factor kappa b (NFkB). Although there is limited data on the impact of $A$. fumigatus on inflammasome signaling, we hypothesize that activation of different inflammasome complexes within the epithelial cells may impact the type of immune response that is activated following A. fumigatus exposures. Most of these studies utilized in vitro epithelial cell models, thereby overlooking the sophisticated cellular interactions that occur between immune cells and the respiratory epithelium. Therefore, further in vivo analysis of epithelial cell responses following A. fumigatus exposures is required to better understand the role of these cells in anti-fungal immunity and the influence fungal-epithelial cell interactions may have on induction of infection, tolerance, and allergy/asthma.

CD8 T cell responses (TNF $\alpha$, IFN- $\gamma$, IL-13, and IL-17) did not appear to be impacted by the method of conidial exposure. $\mathrm{CD} 4^{+} \mathrm{IFN}-\gamma^{+}$and $\mathrm{CD}^{+} \mathrm{TNF}^{+}$counts; however, were increased in mice exposed to WT conidia via aspiration when compared to the acoustical generator. These levels could be altered due to a greater number of conidia within the lungs of mice exposed using aspiration. While murine exposure to different concentrations of conidia has been reported, the quantitative differences arising from this variable remain largely uncharacterized. The difference in these $T_{H} 1$ cells could also be a function of premature activation of conidia as a result of suspension in liquid, though in our studies using rapid sample preparation, no conidial swelling was observed. As discussed previously, metabolic activation results in significant changes in the conidial surface exposing proinflammatory PAMPs that are highly immunogenic, as well as the 
release of numerous allergens $(9,24,52)$. These fungal-host interactions have been shown to result in increases in IFN- $\gamma$ and $\mathrm{TNF} \alpha(3)$.

There is limited information concerning the role of CD8 $\mathrm{T}$ cells in antifungal immune responses. The most significant data obtained from the repeated aspiration exposure model was the induction of a special subset of CD8 T cells, Tc17 cells. Tc17 cells have been implicated in a number of diseases, including viral clearance, systemic lupus erythematosus, anti-tumor responses, contact dermatitis, and pulmonary inflammation (53-58). Tc17 cells have functional plasticity and have been shown to rapidly produce proinflammatory cytokines and chemokines responsible for the recruitment of macrophages, neutrophils, and natural killer cells. A recent study by Nanjappa et al. examined the role of Tc17 cells in vaccine-mediated protection against pneumonia caused by the dimorphic fungal pathogens, Blastomyces dermatiditis and Histoplasma capsulatum, (59). Although B. dermatiditis and $H$. capsulatum have varying pathogenic phases compared to A. fumigatus, the authors showed that Tc17 cells have a nonredundant role in protection against $B$. dermatiditis and $H$. capsulatum infection. Tc17 cells were selectively induced and recruited to the lungs following vaccination with attenuated $B$. dermatiditis, and were required for protection against lung colonization. This response occurred even in the absence of CD4 $\mathrm{T}$ cells. Furthermore, Dectin-1 and $\mathrm{T}_{\mathrm{H}} 1$ cytokines were not required. The Tc17 cells, however, required neutrophils to mediate protection. In Chapter 2 and 3, the lack of Tc17 cells in mice exposed to albino conidia correlated with the decrease in neutrophil numbers (59). Furthermore, these data suggest that Tc17 cells are generated in response to conidia germination. Albino conidia appeared to be cleared before they could germinate in these models, which is consistent with previous data. However, WT conidia showed signs of germination within 4 hours of the final exposure and peaked at 24-48 hours. The extent of germination was closely associated with the influx of Tc17 cells to the lungs of exposed mice. These results were specific to fungi as exposure to silicone beads did not results in specific $\mathrm{T}$ cell responses or IL-17 production. The influx of Tc17 cells also appeared to influence neutrophil recruitment to the airways; a finding that is consistent with previously published reports on the function of IL-17 (60-62). We hypothesize that Tc17 cells, once activated and recruited to the lungs, enhance the recruitment of innate cells upon repeated exposure to the same antigen. However, because of the limited mechanistic data obtained from these studies, the biological relevance of Tc17 cells responses requires further investigation. 
The production of antibody was another variable that may have been influenced by the method of conidial exposure (Appendix Table A2). In these studies, the presence of $A$. fumigatus-specific IgG was examined using ELISA (Chapter 2) and Western blot (Chapter 3). There was minimal IgG production detected from the aspiration exposure animals as determined by ELISA. ELISAs are a sensitive method for analysis of serum antibodies. However, due to the complexity of the protein profile and differential expression/concentrations of proteins in conidial extracts, it is possible that lower concentrations of immunoreactive proteins bound to the plate during the assay reduced assay sensitivity. IgG reactivity to these proteins were likely below the detection limit of the assay, and could not be quantified. In Chapter 3, specific-IgG analysis was assessed using Western blotting. Numerous IgG-reactive bands were visualized using pooled, polyclonal sera from exposed mice. The identity of these bands was not determined by mass spectrometry.

In a study by Chaudary, several of these bands were identified by (19). The most prominent band in our data was a doublet near $40 \mathrm{kD}$. Aspf22 is a protease with a molecular weight of $47.3 \mathrm{kD}$, and appears as a doublet (19). Several small molecular weight proteins were visible in the experiments presented here, which may represent Aspf 7 ( allergen, mw=11.6 kD), Aspf8 (acidic ribosomal protein 2, mw=11.1 kD), and Aspf13 (allergen 13/protease, mw=15.9 $\mathrm{kD})$ (19). Another prominently reactive band was present near $52 \mathrm{kD}$, which may correspond to Aspfl 2 (heat shock protein, $\mathrm{mw}=50.5 \mathrm{kD}$ ) or Aspfl 8 (serine protease, $\mathrm{mw}=52.6 \mathrm{kD}$ ). Furthermore, a predominant allergen associated with A. fumigatus is enolase. The molecular weight of this protein is $47.3 \mathrm{kD}$, which may be the visible band near $40 \mathrm{kD}$ in our studies (19). Aspf5 is a metalloprotease with a molecular weight of $68.7 \mathrm{kD}$, which may correspond to the reactive band near $60 \mathrm{kD}$ in the data presented in Chapter 3 (19). While these are the potential identities of the bands observed in our studies, definitive mass spectrometry analysis of these samples needs to be completed. Total IgE was analyzed in both experiments using ELISA. Total IgE levels were elevated in acoustical generator exposed mice when compared to the aspiration mice. While it may be likely that nose-only inhalation exposure to dry conidia resulted in increased antibody production, due to changes in dose concentration and schedules, it cannot be definitively determined that the reason for these differences was due to dry conidial exposures. A. fumigatus contains numerous allergens; however, the immunoreactivity of many of these proteins remains uncharacterized. The A. fumigatus-specific proteins identified in Chapter 3 
could be useful biomarkers of A. fumigatus-associated adverse health outcomes, leading to the development of more specific and sensitive immunodiagnostic methods.

WT (green), $\triangle a l b 1 /$ albino (white), and $\triangle a r p 2$ (tan) strains from a clinical isolate of $A$. fumigatus were used for exposures in both Chapters 2 and 3. However, data acquired from $\Delta a r p 2$ acoustical generator exposures were not reported due to several confounding factors that made the data difficult to interpret. At the time of sacrifice, several control animals were placed in cages designated for exposed mice at each respective time point. During sample collection, inflammation was observed in the lungs of several control mice, while the lungs of some exposed mice did not appear to have any inflammation. During flow cytometry analysis, distinct populations of eosinophils and $\operatorname{IgE}^{+} \mathrm{B}$ cells were identified in control mice. Historical data showed these cell populations were not present in control mice from either aspiration or acoustical generator exposures from previous experiments. Furthermore, histopathological assessment of several $\Delta a r p 2$ exposed animals from acoustical generator groups showed limited inflammation that was indicative of previous exposures, though it was not similar to lung sections from exposed mice that were placed in the correct cages. Furthermore, there was limited antibody production in these animals. When compared to the data acquired from WT and $\Delta a l b 1$ exposed mice, these observations demonstrated that there appeared to be a problem during the final acoustical generator exposures of $\Delta a r p 2$ mice. As a result, $\Delta a r p 2$ data were removed from the analysis in Chapter 3.

Though the data suggests that the immune responses occurring following exposures to $A$. fumigatus are mixed $\mathrm{T}_{\mathrm{H}} 1$ and $\mathrm{T}_{\mathrm{H}} 2$ responses, definitive conclusions on mechanisms/signaling pathways involved in these responses could not be determined. However, several additional $\mathrm{T}$ cells and cytokines were examined in exposures to albino conidia. T cell production of IL-5 (important in the recruitment of eosinophils), and IL-22 (produced by activated $\mathrm{T}_{\mathrm{H}} 17$ cells) were examined in the BALF. Data indicated each of these cell types were significantly enhanced above control levels in response to repeated aerosol exposures. IL-12 producing $\mathrm{T}$ cells were also examined in the mediastinal lymph nodes to determine if the $\mathrm{T}_{\mathrm{H}} 1$ response that occurred. IL-17 and IL-22 were also examined. These cell types were not examined in mice exposed to WT conidia, or in the aspiration exposures due to previous limitations of the available flow cytometer. However, it would be beneficial to examine these populations to gain further insight into the pathways involved in the A. fumigatus-specific immune responses. A clinical isolate of 
A. fumigatus was used in these studies. However, previous reports have shown that individual isolates produce different proteins, proteases, and allergens. Therefore, additional studies using environmental isolates would likely lead to the identification of the roles each of these proteins play in mediating the immune response to A. fumigatus. This information may aid in determining new targets for anti-fungal therapies.

While fungal conidia make up a large concentration of microbes present in naturally occurring bioaerosols, respirable hyphal fragments are also consistently present in high concentrations. It is largely unknown how exposures to these fragments may impact the pulmonary immune response, though it has been hypothesized that hyphal fragments are the predominant inducers of fungal-specific allergy. Reports by Green et al. have identified these fragments as a major source of aeroallergens (63-65), and in some cases, these fragments can begin to germinate again once inside the host, leading to the production of more allergens. However, there are limited studies that examine the impact of exposures to these fragments in in vitro models. Further studies are required to determine how these fragments may influence the immune responses in both immunocompetent and immunosuppressed models, particularly in the context of allergy and infection, respectively. Therefore, significant information could be obtained following repeated exposures to aerosolized hyphal fragments. Experiments were conducted to examine the hypothesis; however, we were unable to produce sufficient concentrations of 2-5 $\mu \mathrm{m}$ hyphal fragments capable of being deposited in the terminal airways. Further experiments are necessary to determine a better method for hyphal fragmentation.

Additionally, the studies presented in this dissertation examined the impact of a single fungal species; however, other fungi, such as Alternaria, Chaetomium, and Penicillium spp. also constitute a large portion of environmental bioaerosols $(6,8,9,30,63,66)$. Exposures to these fungi have been linked to asthma and allergy, yet the fungal-mediated mechanisms of disease induction have not been adequately addressed in peer reviewed literature. Each of these fungi contains species-specific antigens that may modulate the immune responses against them. Further studies are required to examine these possibilities. Importantly, the acoustical generator dry fungal exposure system and end point analyses have been optimized, allowing for adaptation of the system to examine immune responses following repeated exposures to other occupationally relevant fungi. Additional work will be required to assess the challenges that are 
likely present due to differences in size, shape, metabolism, and nutrient requirements compared to A. fumigatus before these studies can be completed.

In conclusion, the significant findings reported in this dissertation include: 1) identification of a novel cell type, Tc17 cells, which appear to be impacted by conidial melanization and potentially conidia germination, 2) validation that melanin is an important cell wall component that may be responsible for modulating associated immune responses following repeated exposures, 3) observation of numerous immuno-reactive proteins that, upon identification may result in the development of improved immunodiagnostics and/or infection prevention strategies, 4) the presence of melanin does not impact the induction of fungal-specific allergies, and 5) development of a new, dry-fungal aerosol nose-only exposure system that more closely resembles natural human exposures than commonly used exposure protocols. Importantly, the characterization and optimization of the acoustical generator exposure system can be adapted to examine the murine immune responses following repeated exposures to other clinically and environmentally relevant particles. Information obtained from those studies may aid in preventing disease and improving human health. 


\section{REFERENCES}

1. Denning, D. W. 1991. Epidemiology and pathogenesis of systemic fungal infections in the immunocompromised host. Journal of Antimicrobial Chemotherapy 28: 1-16.

2. Dutkiewicz, R., and C. A. Hage. 2010. Aspergillus infections in the critically ill. Proc Am Thorac Soc 7: 204-209.

3. Latgé, J.-P., and W. J. Steinbach. 2009. Aspergillus fumigatus And Aspergillosis. ASM Press.

4. Black, P. N., A. A. Udy, and S. M. Brodie. 2000. Sensitivity to fungal allergens is a risk factor for life-threatening asthma. Allergy 55: 501-504.

5. Chaudhary, N., and K. Marr. 2011. Impact of Aspergillus fumigatus in allergic airway diseases. Clinical and Translational Allergy 1: 4.

6. Crameri, R., M. Weichel, S. Flückiger, A. G. Glaser, and C. Rhyner. 2006. Fungal allergies: a yet unsolved problem. Chemical Immunology and Allergy 91: 121-133.

7. Devereux, G. 2006. The increase in the prevalence of asthma and allergy: food for thought. Nat Rev Immunol 6: 869-874.

8. Kurup, V. P., H. D. Shen, and B. Banerjee. 2000. Respiratory fungal allergy. Microbes and Infection 2: 1101-1110.

9. Simon-Nobbe, B., U. Denk, V. Pöll, R. Rid, and M. Breitenbach. 2008. The spectrum of fungal allergy. International Archives of Allergy and Immunology 145: 58-86.

10. Salo, P. M., A. Calatroni, P. J. Gergen, J. A. Hoppin, M. L. Sever, R. Jaramillo, S. J. Arbes, and D. C. Zeldin. 2011. Allergy-related outcomes in relation to serum IgE: Results from the National Health and Nutrition Examination Survey 2005-2006. The Journal of allergy and clinical immunology 127: 1226-1235.e1227.

11. Bakker-Woudenberg, I. A. J. M. 2003. Experimental models of pulmonary infection. Journal of Microbiological Methods 54: 295-313.

12. Templeton, S. P., A. D. Buskirk, B. J. Green, D. H. Beezhold, and D. Schmechel. 2010. Murine models of airway fungal exposure and allergic sensitization. Medical Mycology 48: 217-228.

13. Bruns, S., O. Kniemeyer, M. Hasenberg, V. Aimanianda, S. Nietzsche, A. Thywißen, A. Jeron, J.-P. Latgé, A. A. Brakhage, and M. Gunzer. 2010. Production of extracellular traps against Aspergillus fumigatus in vitro and in infected lung tissue Is dependent on invading neutrophils and influenced by hydrophobin RodA. PLoS Pathog 6: e1000873.

14. Nessa, K., C. Jarstrand, A. Johansson, and P. Camner. 1997. In vitro interaction of alveolar macrophages and Aspergillus fumigatus. Environmental research 74: 54-60.

15. Schaffiner, A., H. Douglas, and A. Braude. 1982. Selective protection against conidia by mononuclear and against mycelia by polymorphonuclear phagocytes in resistance to Aspergillus. Observations on these two lines of defense in vivo and in vitro with human and mouse phagocytes. The Journal of Clinical Investigation 69: 617-631.

16. Bellanger, A.-P., L. Millon, K. Khoufache, D. Rivollet, I. Bièche, I. Laurendeau, M. Vidaud, F. Botterel, and S. Bretagne. 2009. Aspergillus fumigatus germ tube growth and not conidia ingestion induces expression of inflammatory mediator genes in the human lung epithelial cell line A549. Journal of Medical Microbiology 58: 174-179.

17. Berkova, N., S. Lair Fulleringer, F. Fmnia, D. Huet, M.-C. Wagner, K. Gorna, F. Tournier, O. Ibrahim-Granet, J. Guillot, R. Chermette, P. Boireau, and J.-P. Latg. 2006. 
Aspergillus fumigatus conidia inhibit tumour necrosis factor- or staurosporine-induced apoptosis in epithelial cells. International Immunology 18: 139-150.

18. Borger, P., G. H. Koëter, J. A. Timmerman, E. Vellenga, J. F. Tomee, and H. F. Kauffman. 1999. Proteases from Aspergillus fumigatus induce interleukin (IL)-6 and IL-8 production in airway epithelial cell lines by transcriptional mechanisms. The Journal of Infectious Diseases 180: 1267-1274.

19. Chaudhary, N., J. Staab, and K. Marr. 2010. Healthy human T-Cell responses to Aspergillus fumigatus antigens. PLoS ONE 5: e9036-e9036.

20. Coméra, C., K. André, J. Laffitte, X. Collet, P. Galtier, and I. Maridonneau-Parini. 2007. Gliotoxin from Aspergillus fumigatus affects phagocytos is and the organization of the actin cytoskeleton by distinct signalling pathways in human neutrophils. Microbes and Infection 9: 47-54.

21. Dubourdeau, M., R. Athman, V. Balloy, M. Huerre, M. Chignard, D. Philpott, J.-P. Latg, and O. Ibrahim-Granet. 2006. Aspergillus fumigatus induces innate immune responses in alveolar macrophages through the MAPK pathway independently of TLR2 and TLR4. The Journal of Immunology 177: 3994-4001.

22. Eichner, R. D., M. Al Salami, P. R. Wood, and A. Müllbacher. 1986. The effect of gliotoxin upon macrophage function. International Journal of Immunopharmacology 8: 789-797.

23. Hebart, H., C. Bollinger, P. Fisch, J. Sarfati, C. Meisner, M. Baur, J. Loeffler, M. Monod, J.-P. Latgé, and H. Einsele. 2002. Analysis of T-cell responses to Aspergillus fumigatus antigens in healthy individuals and patients with hematologic malignancies. Blood 100: 4521-4528.

24. Kauffman, H. F., J. F. Tomee, M. A. van de Riet, A. J. Timmerman, and P. Borger. 2000. Protease-dependent activation of epithelial cells by fungal allergens leads to morphologic changes and cytokine production. The Journal of Allergy and Clinical Immunology 105: 1185-1193.

25. Paris, S., E. Boisvieux-Ulrich, B. Crestani, O. Houcine, D. Taramelli, L. Lombardi, and J. P. Latgé. 1997. Internalization of Aspergillus fumigatus conidia by epithelial and endothelial cells. Infection and immunity 65: 1510-1514.

26. Wasylnka, J. A., and M. M. Moore. 2003. Aspergillus fumigatus conidia survive and germinate in acidic organelles of A549 epithelial cells. Journal of Cell Science 116: 1579-1587.

27. Eduard, W. 2009. Fungal spores: a critical review of the toxicological and epidemiological evidence as a basis for occupational exposure limit setting. Critical Reviews in Toxicology 39: 799-864.

28. Ayerst, G. 1969. The effects of moisture and temperature on growth and spore germination in some fungi. Journal of Stored Products Research 5: 127-141.

29. Mitchell, C. G., J. Slight, and K. Donaldson. 1997. Diffusible component from the spore surface of the fungus Aspergillus fumigatus which inhibits the macrophage oxidative burst is distinct from gliotoxin and other hyphal toxins. Thorax 52: 796-801.

30. Portnoy, J. M., C. S. Barnes, and K. Kennedy. 2008. Importance of mold allergy in asthma. Current Allergy and Asthma Reports 8: 71-78.

31. Brakhage, A. A., K. Langfelder, G. Wanner, A. Schmidt, and B. Jahn. 1999. Pigment biosynthesis and virulence. Contributions to Microbiology 2: 205-215. 
32. Butler, M. J., A. W. Day, J. M. Henson, and N. P. Money. 2001. Pathogenic Properties of Fungal Melanins. Mycologia 93: 1-8.

33. Jahn, B., A. Koch, A. Schmidt, G. Wanner, H. Gehringer, S. Bhakdi, and A. A. Brakhage. 1997. Isolation and characterization of a pigmentless-conidium mutant of Aspergillus fumigatus with altered conidial surface and reduced virulence. Infection and Immunity 65: 5110-5117.

34. Tsai, H. F., Y. C. Chang, R. G. Washburn, M. H. Wheeler, and K. J. Kwon Chung. 1998. The developmentally regulated alb1 gene of Aspergillus fumigatus: its role in modulation of conidial morphology and virulence. Journal of bacteriology 180: 3031-3038.

35. Tsai, H. F., R. G. Washburn, Y. C. Chang, and K. J. Kwon Chung. 1997. Aspergillus fumigatus arp1 modulates conidial pigmentation and complement deposition. Molecular microbiology 26: 175-183.

36. Brakhage, A., S. Bruns, A. Thywissen, P. Zipfel, and J. Behnsen. 2010. Interaction of phagocytes with filamentous fungi. Current opinion in microbiology 13: 409-415.

37. Jahn, B., F. Boukhallouk, J. Lotz, K. Langfelder, G. Wanner, and A. A. Brakhage. 2000. Interaction of human phagocytes with pigmentless Aspergillus conidia. Infection and Immunity 68: 3736-3739.

38. Hamilton, A. J., and B. L. Gomez. 2002. Melanins in fungal pathogens. Journal of Medical Microbiology 51: 189-191.

39. Jacobson, E. S. 2000. Pathogenic roles for fungal melanins. Clinical Microbiology Reviews 13: 708-717.

40. Wheeler, M. H., and A. A. Bell. 1988. Melanins and their importance in pathogenic fungi. Current Topics in Medical Mycology 2: 338-387.

41. Thywiben, A., T. Heinekamp, H.-M. Dahse, J. Schmaler-Ripcke, S. Nietsche, P. F. Zipfel, and A. A. Brakhage. 2011. Conidial dihydroxynaphthalene melanin of the human pathogenic fungus Aspergillus fumigatus interferes with the host endocytosis pathway. Frontiers in Microbiology 2.

42. Volling, K., A. Thywissen, A. A. Brakhage, and H. P. Saluz. 2011. Phagocytosis of melanized Aspergillus conidia by macrophages exerts cytoprotective effects by sustained PI3K/Akt signalling. Cellular Microbiology 13: 1130-1148.

43. Wasylnka, J. A., and M. M. Moore. 2002. Uptake of Aspergillus fumigatus conidia by phagocytic and nonphagocytic cells in vitro: quantitation using strains expressing green fluorescent protein. Infection and Immunity 70: 3156-3163.

44. Zhang, Z., R. Liu, J. A. Noordhoek, and H. F. Kauffiman. 2005. Interaction of airway epithelial cells (A549) with spores and mycelium of Aspergillus fumigatus. Journal of Infection 51:375-382.

45. Robinson, B. W., T. J. Venaille, A. H. Mendis, and R. McAleer. 1990. Allergens as proteases: an Aspergillus fumigatus proteinase directly induces human epithelial cell detachment. The Journal of Allergy and Clinical Immunology 86: 726-731.

46. Bäckhed, F., and M. Hornef. 2003. Toll-like receptor 4-mediated signaling by epithelial surfaces: necessity or threat? Microbes and Infection 5: 951-959.

47. Balloy, V., J.-M. Sallenave, Y. Wu, L. Touqui, J.-P. Latg, M. Si Tahar, and M. Chignard. 2008. Aspergillus fumigatus-induced interleukin-8 synthesis by respiratory epithelial cells is controlled by the phosphatidylinositol 3-kinase, p38 MAPK, and ERK1/2 pathways and not by the toll-like receptor-MyD88 pathway. Journal of biological chemistry 283: 30513-30521. 
48. Zhao, J., and X.-Y. Wu. 2008. Aspergillus fumigatus antigens activate immortalized human corneal epithelial cells via Toll-Like Receptors 2 and 4. Current Eye Research 33: 447-454.

49. Guo, H., and X. Wu. 2009. Innate responses of corneal epithelial cells against Aspergillus fumigatus challenge. FEMS Immunology \& Medical Microbiology 56: 88-93.

50. Besnard, A.-G., D. Togbe, I. Couillin, Z. Tan, S. G. Zheng, F. Erard, M. Le Bert, V. Quesniaux, and B. Ryffel. 2012. Inflammasome-IL-1-Th17 response in allergic lung inflammation. Journal of Molecular Cell Biology 4: 3-10.

51. Saïd-Sadier, N., E. Padilla, G. Langsley, and D. M. Ojcius. 2010. Aspergillus fumigatus stimulates the NLRP3 Inflammasome through a pathway requiring ROS production and the Syk tyrosine kinase. PLoS ONE 5: e10008.

52. Shen, H.-D., M. F. Tam, R.-B. Tang, and H. Chou. 2007. Aspergillus and Penicillium allergens: focus on proteases. Current Allergy and Asthma Reports 7: 351-356.

53. Garcia-Hernandez, M. d. 1. L., H. Hamada, J. B. Reome, S. K. Misra, M. P. Tighe, and R. W. Dutton. 2010. Adoptive Transfer of Tumor-Specific Tc17 Effector T Cells Controls the Growth of B16 Melanoma in Mice. The Journal of Immunology 184: 4215-4227.

54. Hamada, H., M. d. 1. L. Garcia-Hernandez, J. B. Reome, S. K. Misra, T. M. Strutt, K. K. McKinstry, A. M. Cooper, S. L. Swain, and R. W. Dutton. 2009. Tc17, a Unique Subset of CD8 T Cells That Can Protect against Lethal Influenza Challenge. The Journal of Immunology 182: 3469-3481.

55. Henriques, A., L. Inês, M. Couto, S. Pedreiro, C. Santos, M. Magalhães, P. Santos, I. Velada, A. Almeida, T. Carvalheiro, P. Laranjeira, J. M. Morgado, M. L. Pais, J. A. P. d. Silva, and A. Paiva. 2010. Frequency and functional activity of Th17, Tc17 and other Tcell subsets in Systemic Lupus Erythematosus. Cellular Immunology 264: 97-103.

56. Yeh, N., N. L. Glosson, N. Wang, L. Guindon, C. McKinley, H. Hamada, Q. Li, R. W. Dutton, P. Shrikant, B. Zhou, R. R. Brutkiewicz, J. S. Blum, and M. H. Kaplan. 2010. Tc17 Cells Are Capable of Mediating Immunity to Vaccinia Virus by Acquisition of a Cytotoxic Phenotype. The Journal of Immunology 185: 2089-2098.

57. Yen, H.-R., T. J. Harris, S. Wada, J. F. Grosso, D. Getnet, M. V. Goldberg, K.-L. Liang, T. C. Bruno, K. J. Pyle, S.-L. Chan, R. A. Anders, C. L. Trimble, A. J. Adler, T.-Y. Lin, D. M. Pardoll, C.-T. Huang, and C. G. Drake. 2009. Tc17 CD8 T Cells: Functional Plasticity and Subset Diversity. The Journal of Immunology 183: 7161-7168.

58. Zhao, Y., A. Balato, R. Fishelevich, A. Chapoval, D. L. Mann, and A. A. Gaspari. 2009. Th17/Tc17 infiltration and associated cytokine gene expression in elicitation phase of allergic contact dermatitis. British Journal of Dermatology 161: 1301-1306.

59. Nanjappa, S. G., E. Heninger, M. Wüthrich, D. J. Gasper, and B. S. Klein. 2012. Tc17 cells mediate vaccine immunity against lethal fungal pneumonia in immune deficient hosts lacking CD4 T+ cells. PLoS Pathog 8: e1002771.

60. Korn, T., E. Bettelli, M. Oukka, and V. K. Kuchroo. 2009. IL-17 and Th17 Cells. Annual Review of Immunology 27: 485-517.

61. Lindén, A., and M. Adachi. 2002. Neutrophilic airway inflammation and IL-17. Allergy 57: 769-775.

62. Nembrini, C., B. J. Marsland, and M. Kopf. 2009. IL-17-producing T cells in lung immunity and inflammation. Journal of allergy and clinical immunology 123: 986-994. 
63. Green, B. J., T. Z. Mitakakis, and E. R. Tovey. 2003. Allergen detection from 11 fungal species before and after germination. The Journal of Allergy and Clinical Immunology 111: 285-289.

64. Green, B. J., J. K. Sercombe, and E. R. Tovey. 2005. Fungal fragments and undocumented conidia function as new aeroallergen sources. The Journal of Allergy and Clinical Immunology 115: 1043-1048.

65. Green, B. J., E. R. Tovey, J. K. Sercombe, F. M. Blachere, D. H. Beezhold, and D. Schmechel. 2006. Airborne fungal fragments and allergenicity. Medical Mycology 44 Suppl 1: S245-255.

66. Curtis, L., A. Lieberman, M. Stark, W. Rea, and M. Vetter. 2004. Adverse health effects of indoor molds. Journal of Nutritional \& Environmental Medicine 14: 261-274. 


\section{APPENDIX}

Figure A1.

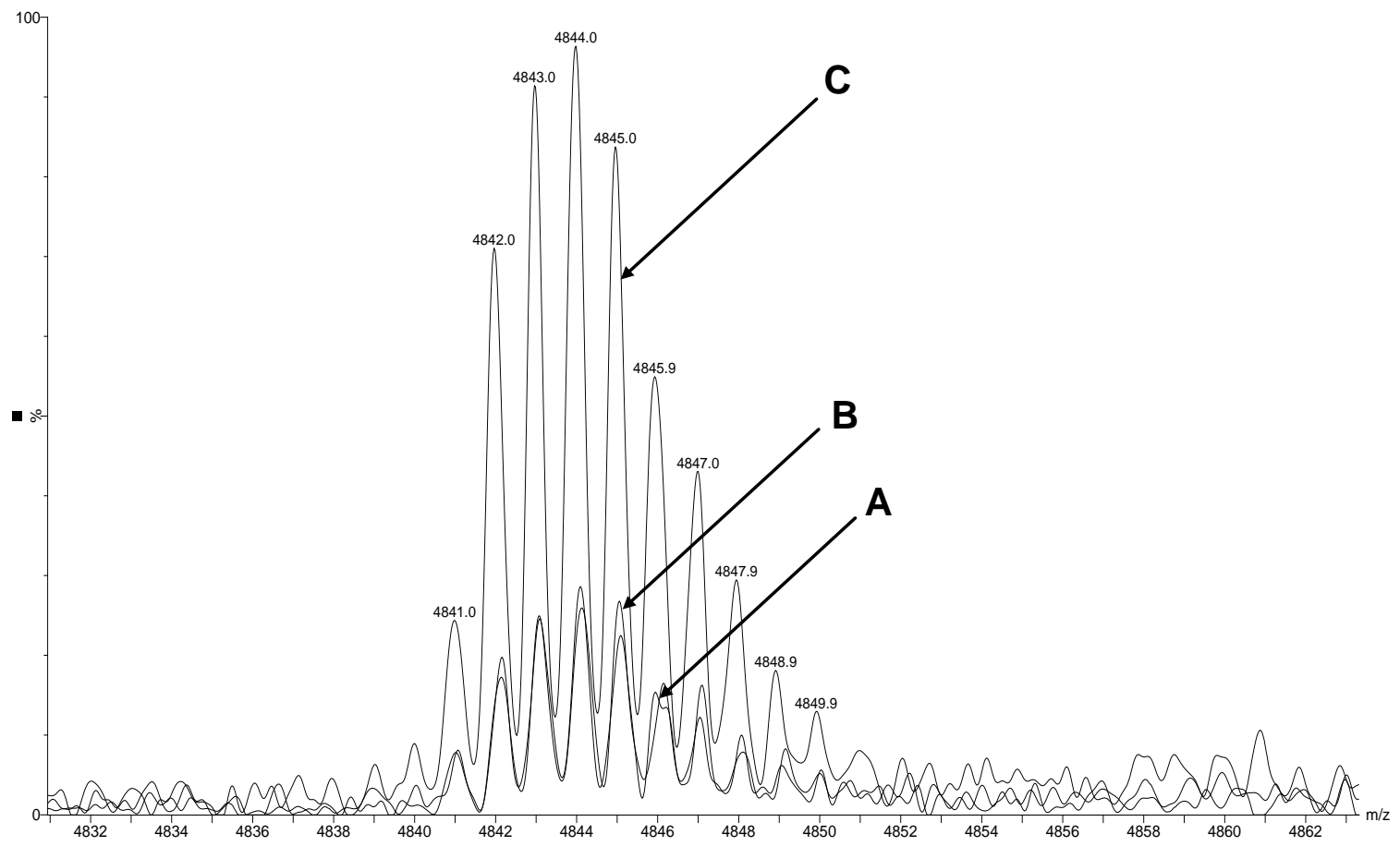

Figure A1. Melanin inhibits peak abundance. +MALDI qTOF MS mass spectra of the multicharged peak at $m / z, 4840$ illustrating peak abundance inhibition caused by melanin. A) $A$. fumigatus WT spectrum, B) $\Delta a r p 2$ spectrum, and C) $\Delta a l b 1$ spectrum. 


\section{Table A1.}

\begin{tabular}{cccc}
\hline \multicolumn{5}{c}{ Summary of Conidia Germination in Lung Tissue } \\
\hline Exposure Group & Total Conidia & Swollen Conidia & Germ Tubes \\
\hline Aspergillus fumigatus WT & 4011 & $281(7.0 \%)$ & $29(0.7 \%)$ \\
Aarp2 & 3625 & $138(3.8 \%)$ & $19(0.5 \%)$ \\
& 1210 & $18(1.5 \%)$ & $0(0.0 \%)$ \\
\hline
\end{tabular}

Supplementary Table S1. Summary of conidial germination in lung tissue. Conidia were quantified from 150 different fields of view at 40X magnification for each set of lungs. Aspergillus fumigatus WT and $\triangle$ alb1 $N=4$ mice, $\Delta$ arp $2 N=5$ mice. 


\section{Figure A3}

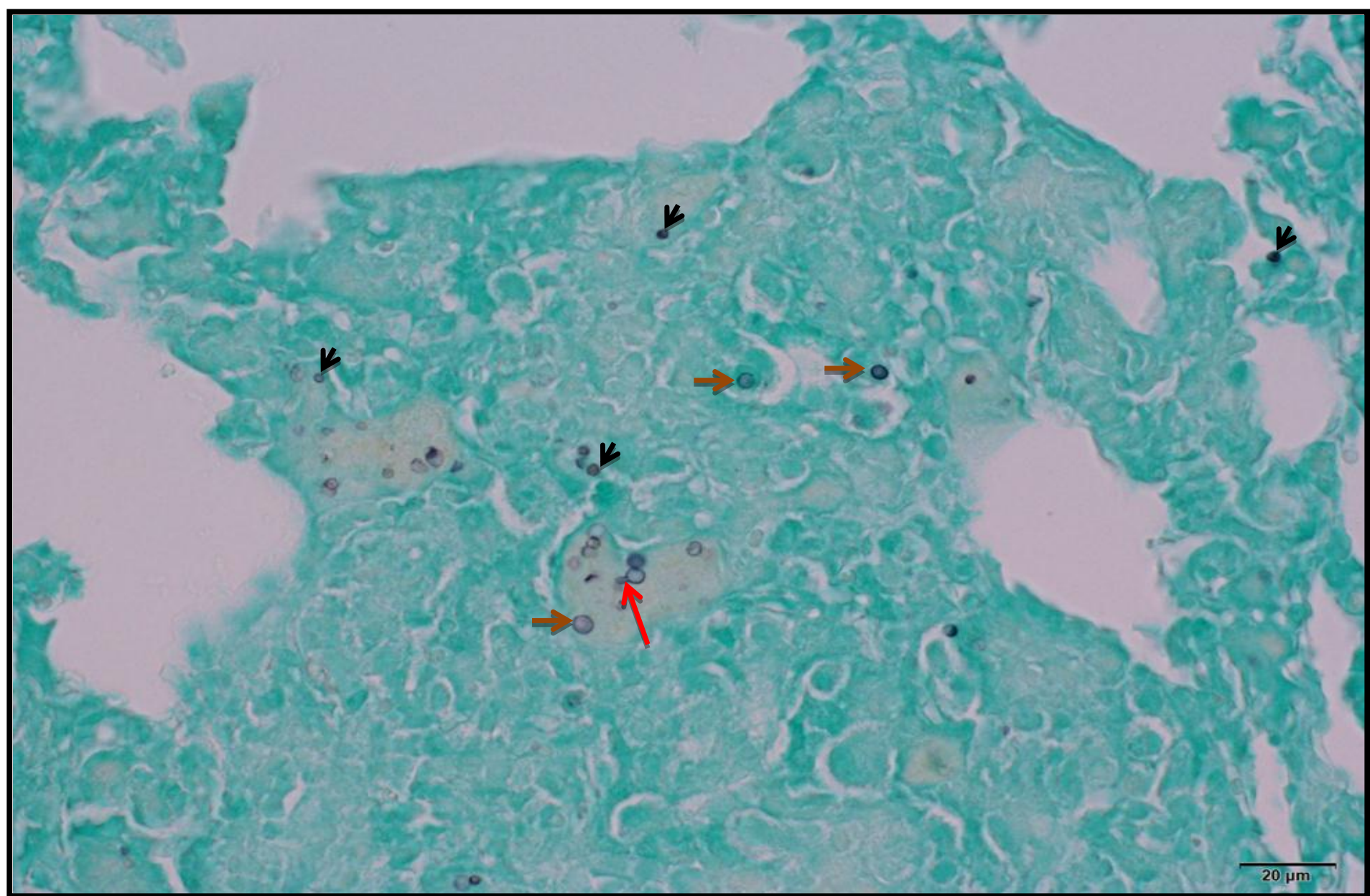

Figure A3: 80X magnification of GMS stained lung section from mouse exposed to WT conidia. Black arrows indicate resting conidia. Orange arrows indicated swollen conidia (2-3X the size of resting conidia). Red arrow indicates germ tube formation. 


\section{Figure A4}
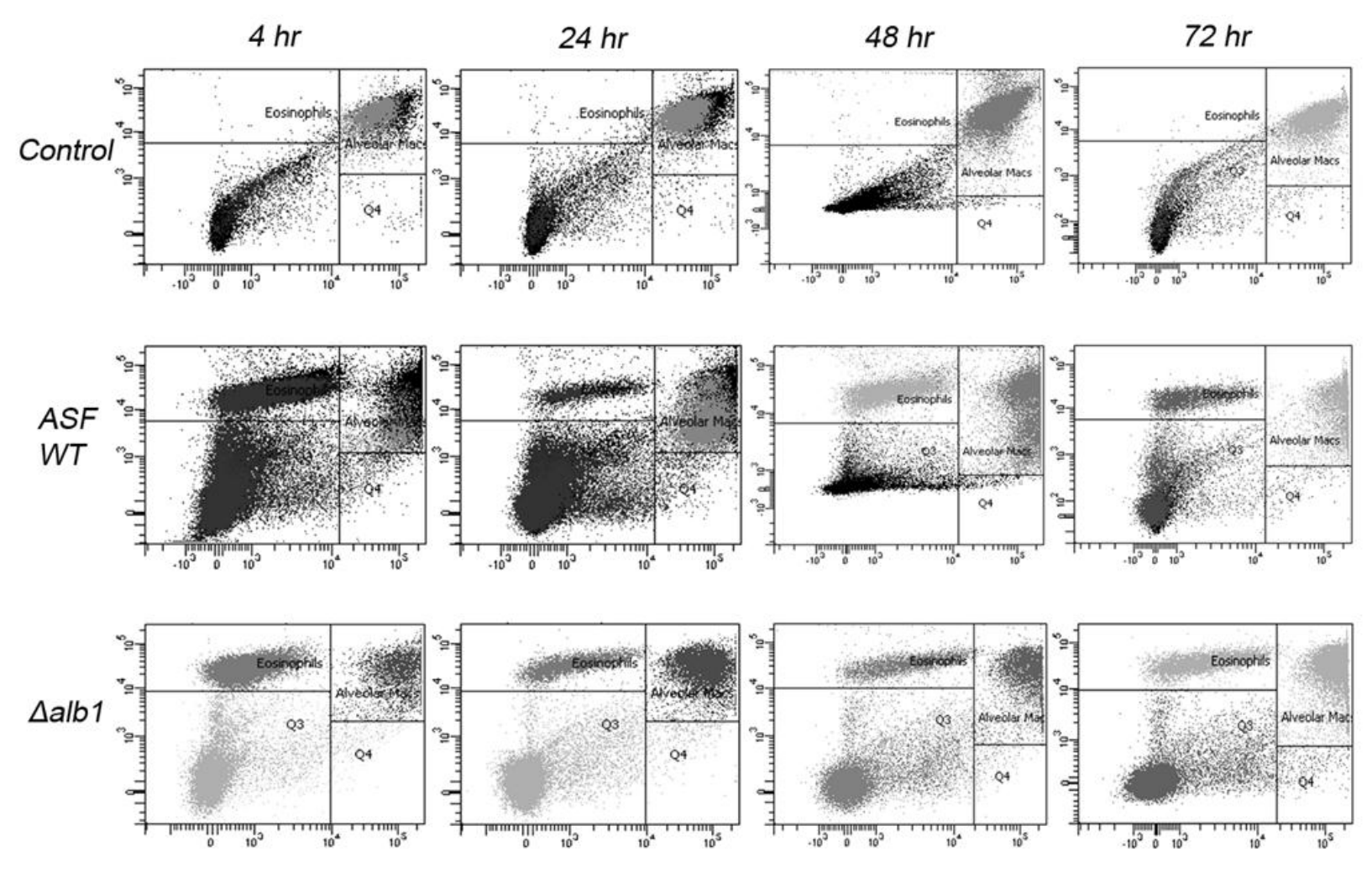

Figure A4. Flow cytometric dot plot representation of alveolar macrophages. Representative images from exposed mice as indicated time points were gated for alveolar macrophages (upper right quadrant) and eosinophils (upper left quadrant). As shown in control mice at each examined time point, alveolar macrophages remained consistent between mice and times. In mice exposed to WT conidia, there was an immediate downward shift in this population (potential M2 cells), which remained evident until 72 hours after the final exposure. Albino exposed mice also exhibited this shift, albeit less pronounced than WT exposed mice. 


\section{Exposed ASF WT Exposed ASF WT}

\section{Control}

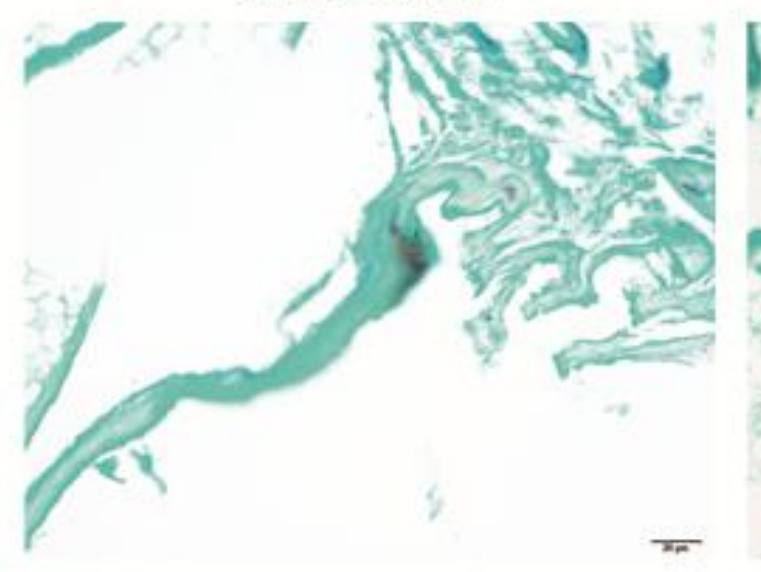

\section{Asymptomatic} Symptomatic
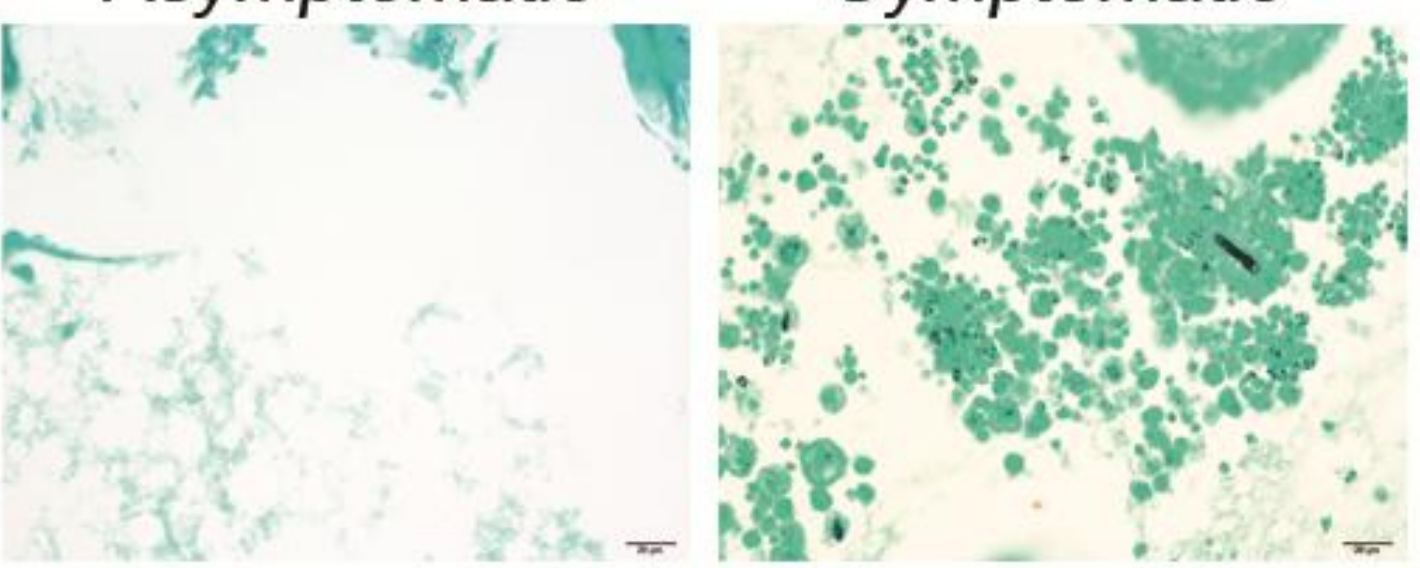

Figure A5. Ear infection analysis of torticollis mice. Representative GMS stained cross sections of the middle/inner ear of control or exposed mice at 40X magnification. Moderate inflammation accompanying germinating conidia are apparent in the inner ear of mice with torticollis. These results were not observed in control or asymptomatic mice. Out of 40 mice, only 2 developed symptoms of torticollis following exposures. Inner ear infections likely resulted from conidia traveling into the eustachain tubes during exposures. 
Figure A6

\section{BALF T cell Cytokine Production}
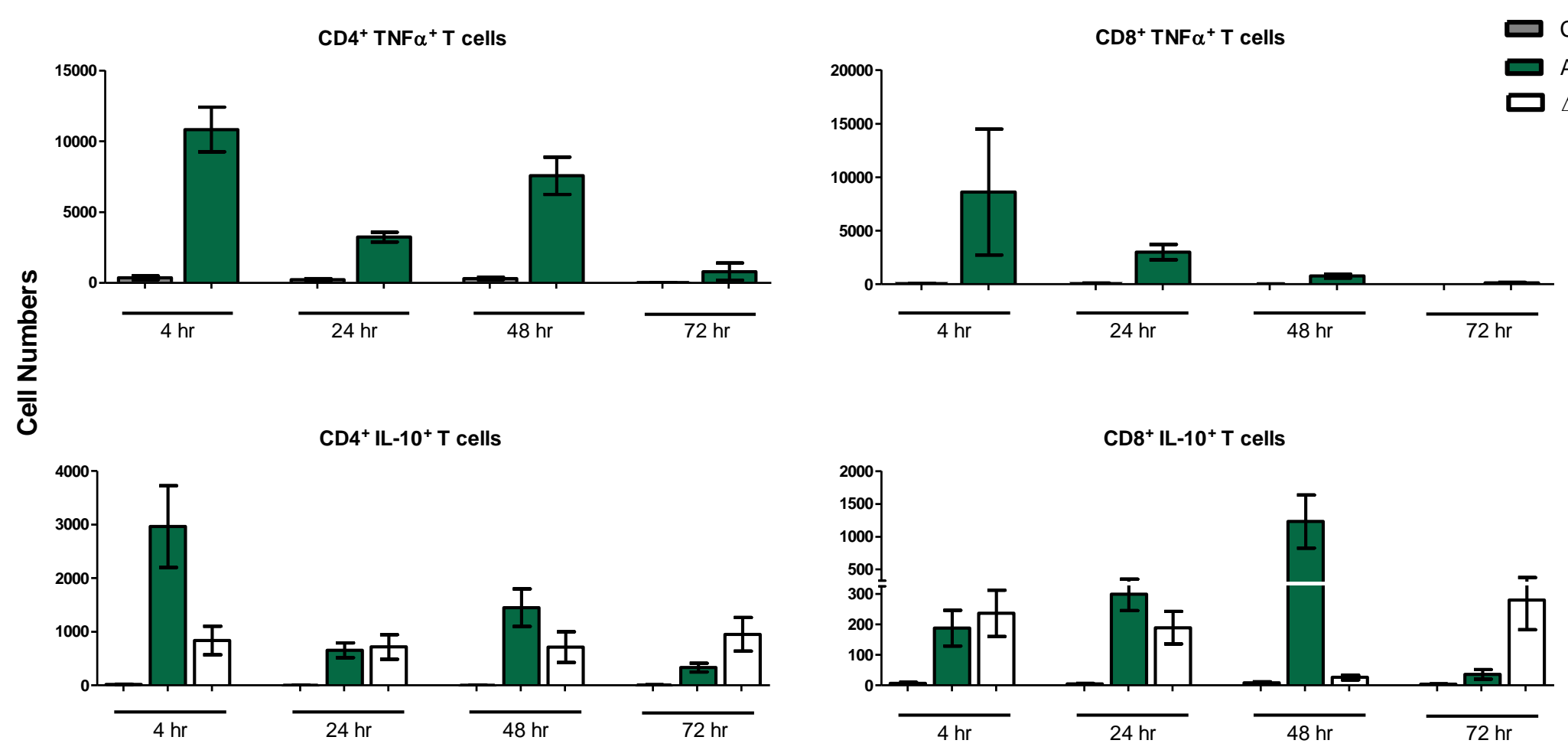

Exposure Groups

Figure A6. Flow cytometric analysis of intracellular cytokine production in the BALF. CD4 and CD8 T cells were quantified by multiplying the frequency of each individual cell population by the total cell counts. Mice were sacrificed at the indicated time points after the $8^{\text {th }}$ exposure. Data are presented as the average \pm standard error of measure. $N=7-10$ mice/group per time point. 
Figure A7

\section{Lung-Associated Lymph Node T cell \\ Cytokine Production}

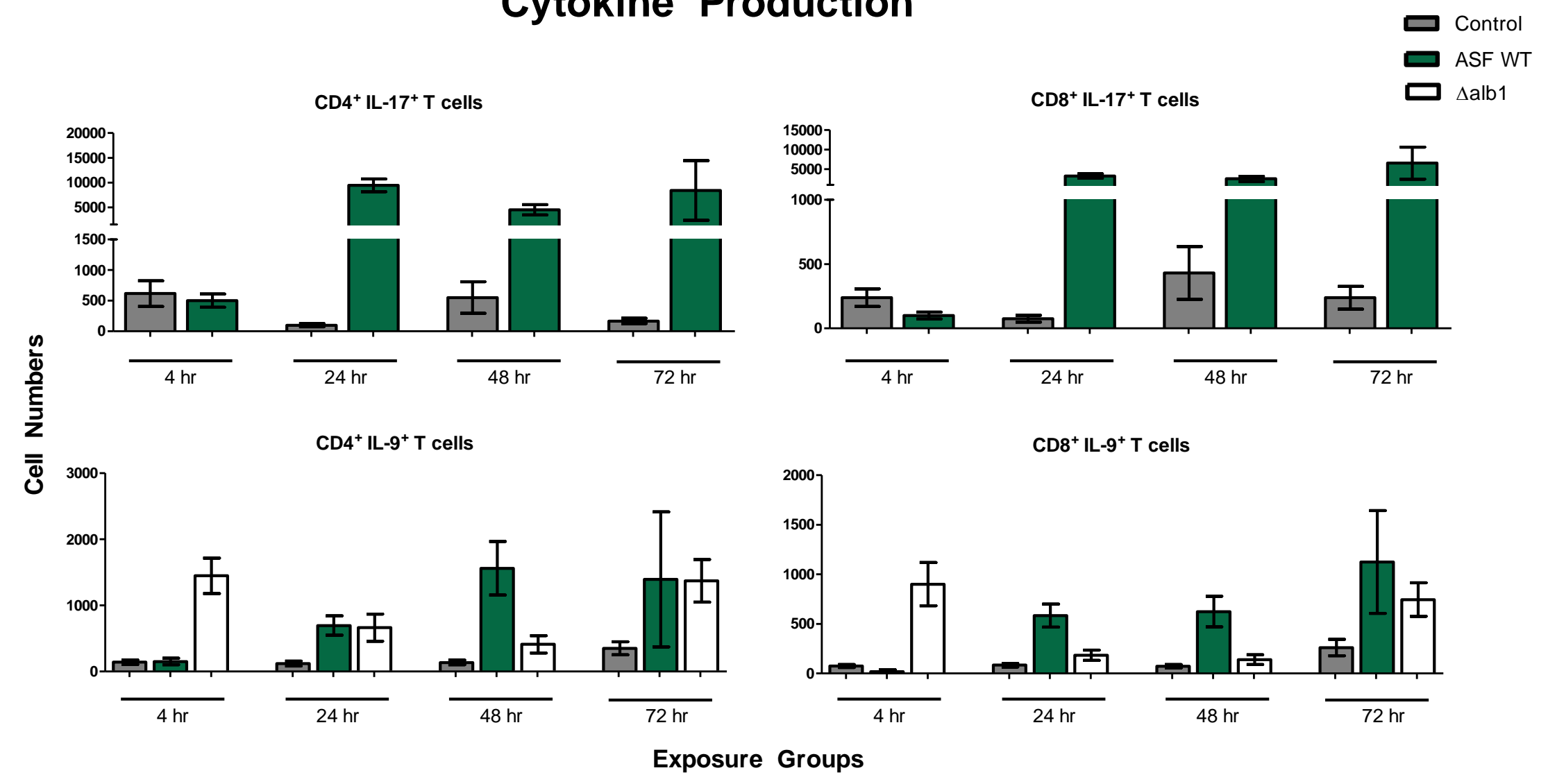

Figure A7. Intracellular cytokine flow cytometric analysis of the mediastinal lymph nodes. CD4 and CD8 T cells were quantified by multiplying the frequency of each individual cell population by the total cell counts. Data are presented as the average \pm standard error of measure. $N=7-10$ mice/group per time point. 
Figure A8

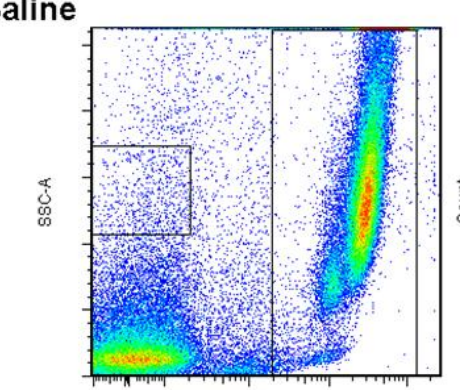

Comp-PerCP-A: CD45
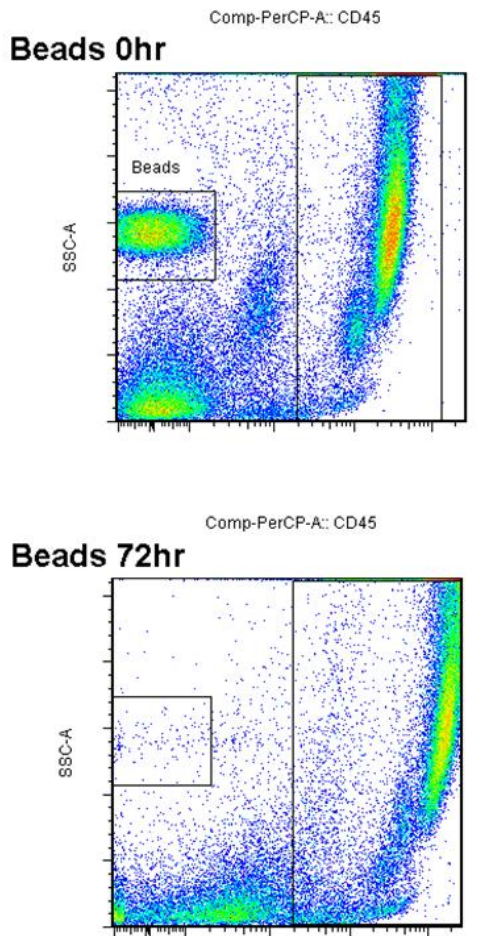

Comp-FITC-A: LY-6G

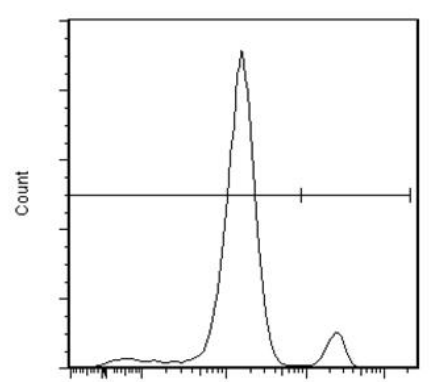

Comp-FITC-A: LY-60
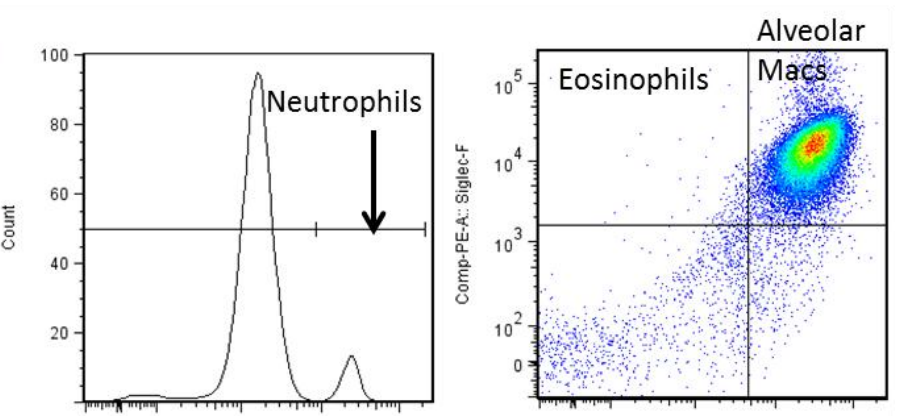

Comp-APC-A: CD11c

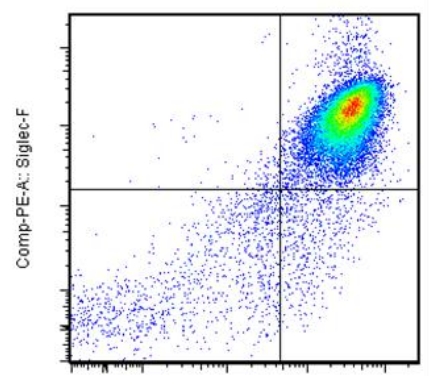

Comp-APC-A: CD11
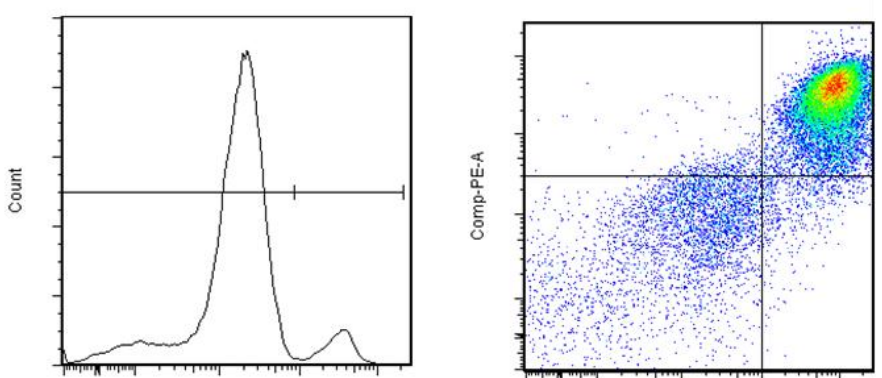

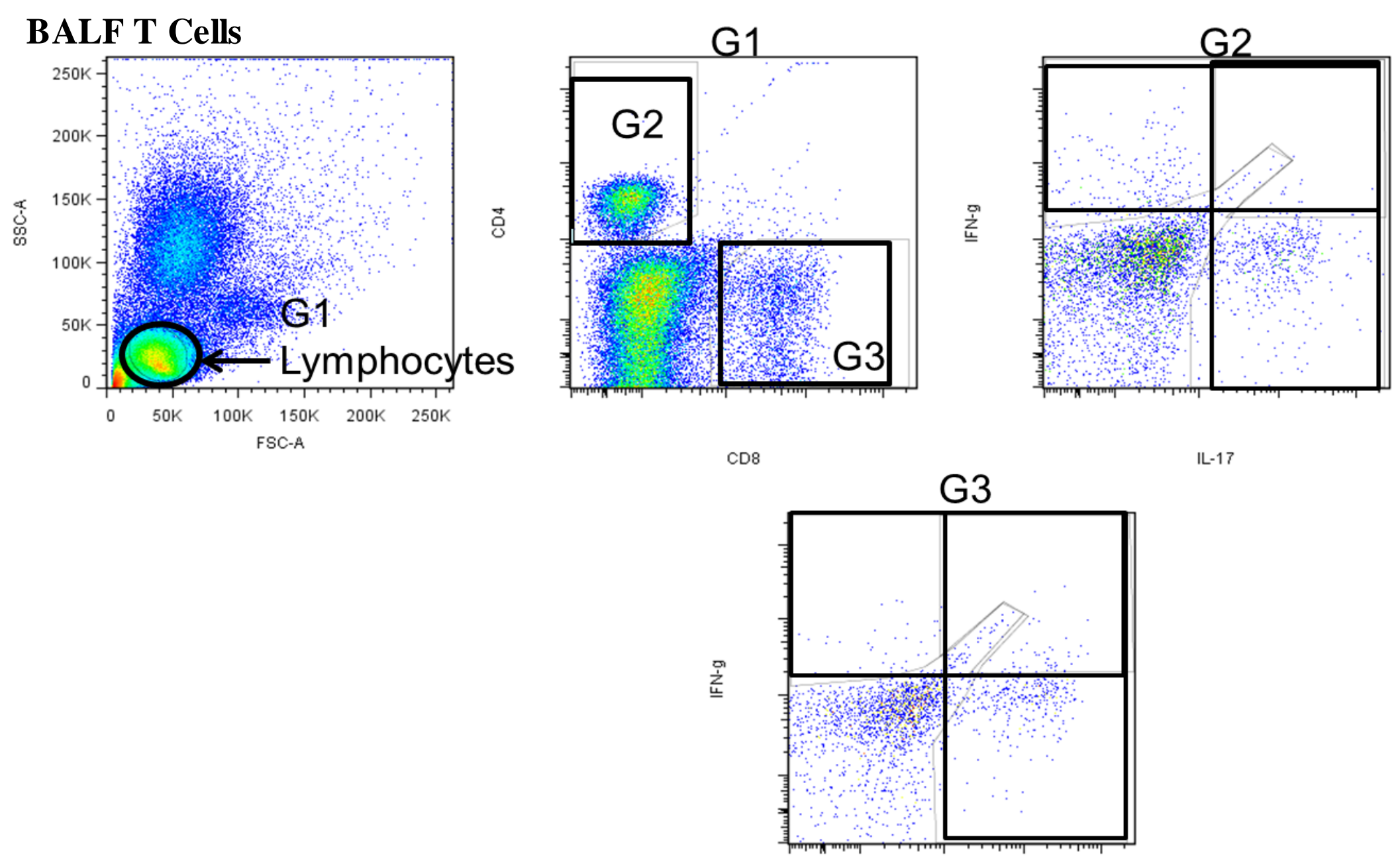

IL-17

IL-17 
Saline

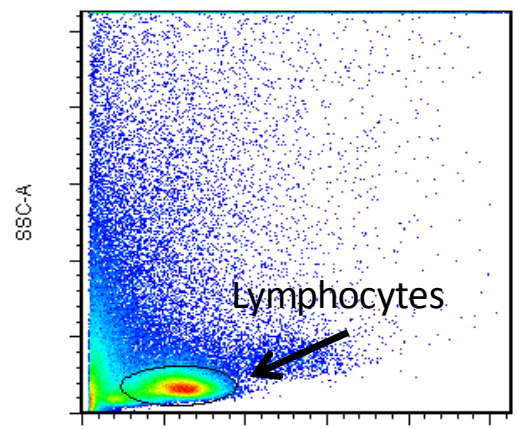

FSC-A

$24 \mathrm{hr}$ Beads

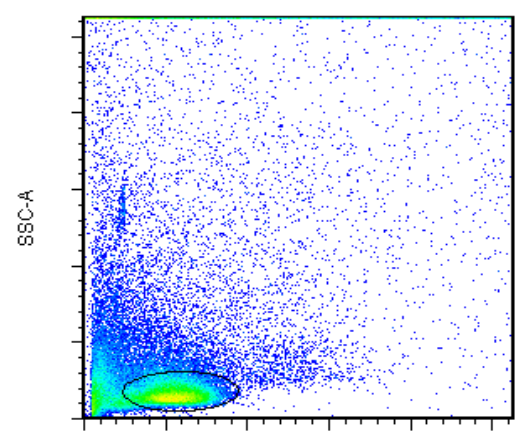

$72 \mathrm{hr}$ Beads

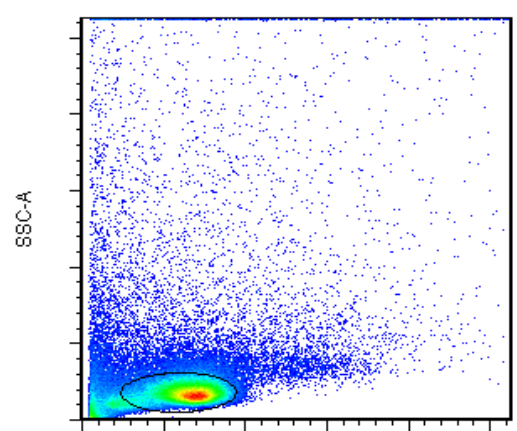

$\mathbf{L N}$

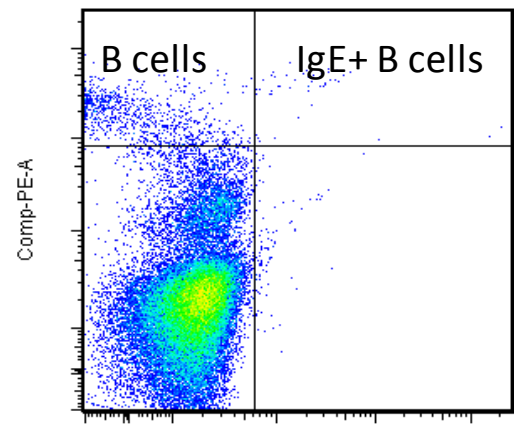

Comp-FITC-A

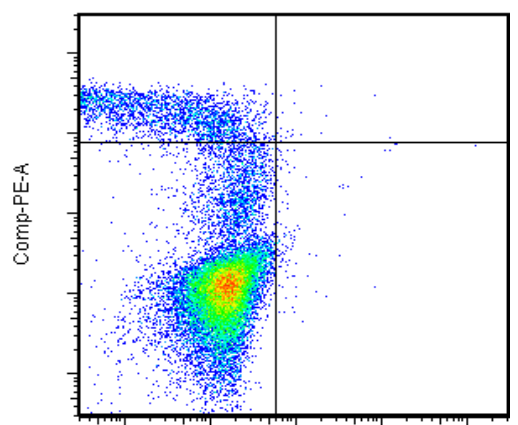

Comp-FITC-A

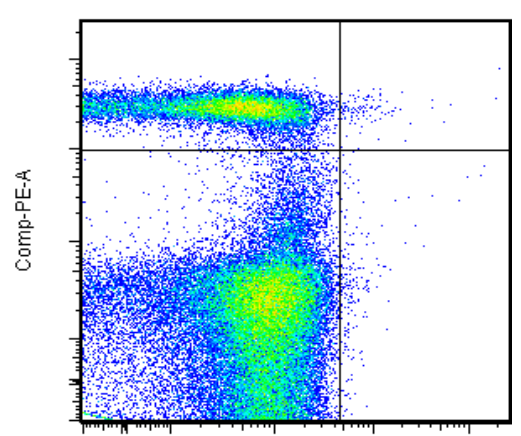

Figure A8. Particulate responses in the BALF and LN following repeated exposures to $2 \mu \mathrm{m}$ silicone beads and flow cytometry gating. Data was acquired at 0, 4, 24, 48, and 72 hours post final exposures. Representative data are shown. $N=5$ mice/group/ time point. As observed in the dot plots, limited responses occurred following bead exposures, indicating the results presented in Chapter 3 are specific to fungi. 


\section{Figure A9}

\section{Tc17 cells and Germination}
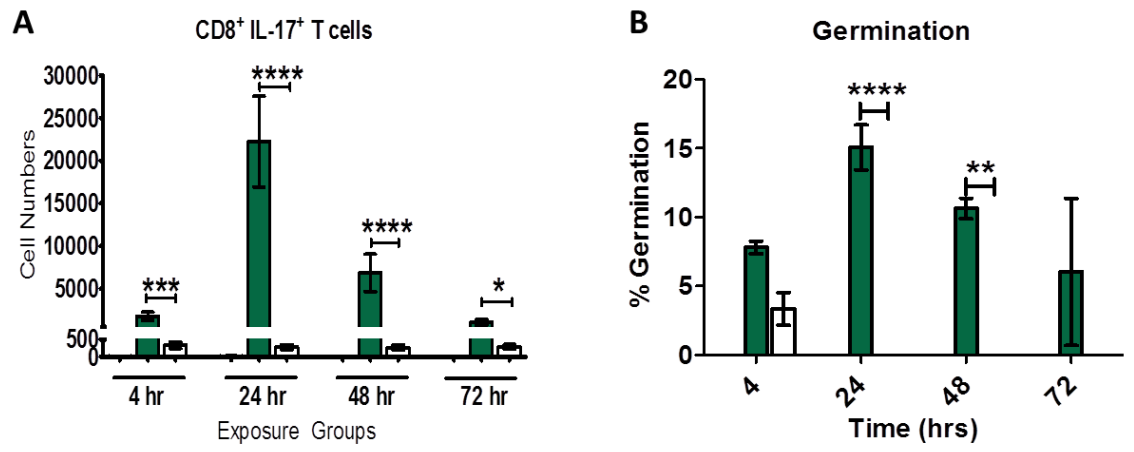

C Correlation Between WT Germination and Tc17 Cells

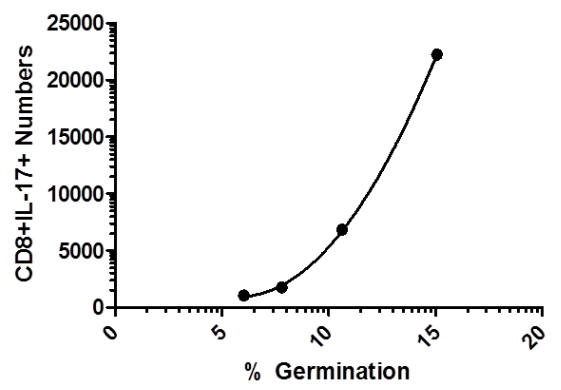

Figure A9. Correlation between Tc17 cells and Germination. A) Tc17 cell numbers at different time points for WT (green bars) and Aalbl (white) exposed mice (n=7-10 mice/group). B) Quantification of germination (swollen conidia + germ tube formation) over time ( $n=3$ mice/group). Data are presented as the average \pm standard error of measure. $* * * * P \leq 0.0001$, $* * * P \leq 0.001, * * P \leq 0.01, * P \leq 0.05$. C) A second order quadratic non-linear regression analysis comparing $T c 17$ cells vs. percent of germination. $R^{2}=0.9998$ indicating a close correlation between Tc17 cells and germination. 
Appendix Table A2

\begin{tabular}{|c|c|c|}
\hline \multicolumn{3}{|c|}{ Comparison to aspiration } \\
\hline End Points & $W T$ & $\Delta a l b 1$ \\
\hline Total Cell Counts & Comparable & Comparable \\
\hline Eosinophils & Comparable & Decreased \\
\hline Neutrophils & Comparable & Decreased \\
\hline $\mathrm{CD}^{+}{ }^{+} \mathrm{T}$ cells & Comparable & Comparable \\
\hline $\mathrm{CD}^{+}{ }^{+} \mathrm{TNF}^{+}{ }^{+} \mathrm{T}$ cells & Decreased & $\mathrm{N} / \mathrm{A}$ \\
\hline $\mathrm{CD}^{+}{ }^{+} \mathrm{IFN}-\gamma^{+} \mathrm{T}$ cells & Decreased & Slightly Increased \\
\hline CD4 ${ }^{+} \mathrm{IL}-17^{+} \mathrm{T}$ cells & Comparable & Comparable \\
\hline $\mathrm{CD}^{+} \mathrm{T}$ cells & Comparable & Comparable \\
\hline $\mathrm{CD}^{+}{ }^{+} \mathrm{TNF}^{+}{ }^{+} \mathrm{T}$ cells & Comparable & $\mathrm{N} / \mathrm{A}$ \\
\hline CD8 ${ }^{+}$IFN- $\gamma^{+}$T cells & Comparable & Comparable \\
\hline CD8 ${ }^{+} \mathrm{IL}-17^{+} \mathrm{T}$ cells & Comparable & Comparable \\
\hline Conidial Numbers & Comparable & Comparable \\
\hline Germination & Comparable & Comparable \\
\hline Antibody responses & Detectable & Detectable \\
\hline
\end{tabular}

Table A2: Endpoint comparisons of dry aerosol exposures vs. aspiration exposures. Those highlighted in red indicate dry exposure responses that were decreased compared to aspiration responses. Those highlighted in green indicate dry exposure responses that were increased in comparison to aspiration exposures. 


\section{CURRICULUM VITAE}

\section{AMANDA D. BUSKIRK}

Residence:

310 Rolling Hills Village

Morgantown, WV 26508

Ph: 301-697-0537
Lab/Office:

CDC/NIOSH/HELD/ACIB

$\mathrm{H}-2506$

Ph: (304) 285-5721 ext. 4

\section{PROFESSIONAL INTERESTS}

Developing mouse models of infection, autoimmunity and/or cancer; pulmonary immunology; $\mathrm{T}$ cells (host-mediated protection, induction, regulation, and plasticity); leukocyte trafficking; and antigen processing and presentation.

\section{EDUCATION}

2005-Present

$2005-2010$

2000-2004

HONORS/AWARDS

2012
WEST VIRGINIA UNIVERSITY

Morgantown, WV

Ph.D. Immunology and Microbial Pathogenesis

WEST VIRGINIA UNIVERSITY

Morgantown, WV

M.S. Biomedical Sciences

Thesis title: The Effects of Fungal Pigments on Matrix-Assisted

Laser Desorption/Ionization Time-of-Flight Mass Spectrometry

FROSTBURG STATE UNIVERSITY

Frostburg, MD

B.S. Biology

Minor, Chemistry

Concentration, Biotechnology
WEST VIRGINIA UNIVERSITY

Van Liere Convocation and Research Day

First place, poster session: Basic Sciences 2003-06 

Associate Member

Greenbrier Valley Chapter, WV

2007

WEST VIRGINIA UNIVERSITY

Graduate Travel Award

To attend the American Society for Mass Spectrometry annual conference

\section{RESEARCH EXPERIENCE}

2005-Present

CENTERS FOR DISEASE CONTROL AND PREVENTION NATIONAL INSTITUTE OF OCCUPATIONAL SAFETY AND HEALTH

Morgantown, WV

Health Effects Laboratory Division

Allergy and Clinical Immunology Branch

Regular Fellow

- Developed a mouse model of repeated exposures to fungi and characterized the pulmonary immune responses.

- Worked with colleagues to develop a protocol for rapid identification of different fungal and bacterial species using mass spectrometry, including darkly pigmented fungi that were unable to be identified, previously.

WINDBER RESEARCH INSTITUTE

Windber, PA

Proteomics Laboratory

Student Intern/Regular Fellow I

- Identified potential biomarkers of breast cancer from patient samples by western blot analysis.

- Processed patient breast cancer samples for 2-D gel electrophoresis and mass spectrometry to identify biomarkers of disease.

\section{TECHNICAL EXPERIENCE}

Multi-parameter flow cytometry

Mouse handling - pulmonary antigen exposures/immunizations/dissection

Development of animal models

Mass spectrometry (MALDI-TOF)

Protein purification 
Proteomic analysis

Tissue culture

Fungal culture

ELISA

Allergen identification

\section{PRESENTATIONS/CONFERENCES}

1. American Thoracic Society, San Francisco, CA. May 2012. "A Nose-Only Inhalation Exposure System For The Delivery Of Dry Fungal Spores (Aspergillus fumigatus)”

2. * Van Liere Convocation and Research Day, Morgantown, WV. March 2012. "The Foils of Fungal Melanins"

3. $98^{\text {th }}$ Annual Meeting of the American Association of Immunologists, San Francisco, CA. May 2011. "Variability in murine airway immune responses to clinical and environmental isolates of Aspergillus conidia"

4. $110^{\text {th }}$ General Meeting of the American Society for Microbiology, San Diego, CA. May 2010 "Murine Pulmonary CD4 and CD8 T-cell Responses to Aspergillus conidia are Species-Dependent"

5. * Van Liere Convocation and Research Day, Morgantown, WV. April 2009. "Developing and Characterizing a Mouse Model of Dry Mycoaerosol Exposures"

6. Joint Statistical Meeting, Denver, CO. August 2008. "Classification of Penicillium Strains Using Discriminant Analysis"

7. $56^{\text {th }}$ ASMS Conference on Mass Spectrometry and Allied Topics, Denver, CO. June 2008. "Discrimination of Penicillium isolates by Matrix Assisted Laser Desorption/ Ionization Time- of- Flight Mass Spectrometry Fingerprinting”

8. * 56 $6^{\text {th }}$ ASMS Conference on Mass Spectrometry and Allied Topics, Denver, CO. June 2008. "Discrimination of Aspergillus isolates at the species and strain level by MALDITOF mass spectrometry fingerprinting”

9. * Van Liere Convocation and Research Day, Morgantown, WV. April 2008. "Developing and Characterizing a Mouse Model Of Fungal Exposure To Dry Spores" 
10. American Academy of Asthma Allergy and Immunology, Philadelphia, PA. March 2008. "Identifying fungal isolates using matrix-assisted laser desorption/ionization time-of-flight mass spectrometry"

11. * 55 ${ }^{\text {th }}$ ASMS Conference on Mass Spectrometry and Allied Topics, Indianapolis, ID. June 2007. "Investigation of Sample Preparation Strategies for MALDI- TOF MS Analysis of Mycobacterial Proteins"

12. $55^{\text {th }}$ ASMS Conference on Mass Spectrometry and Allied Topics, Indianapolis, ID. June 2007. "MALDI-TOF MS Fingerprinting of Fungal Isolates"

13. $55^{\text {th }}$ ASMS Conference on Mass Spectrometry and Allied Topics, Indianapolis, ID. June 2007. "Identification of Mycobacteria and Mycobacteria Biomarker Proteins by Novel Biological Preparation Combined with Tandem Mass Spectrometry"

14. * Van Liere Convocation and Research Day, Morgantown, WV. April 2007. "Optimization of MALDI-TOF Mass Spectrometry Analysis of Mycobacteria"

NOTE: * Indicates first author

\section{PUBLICATIONS}

1. A.D. Buskirk, S.P. Templeton, A.P. Nayak, B.F. Law, B.J. Green, and D.H. Beezhold. "Melanin modulates the pulmonary immune response to Aspergillus fumigatus conidia". Manuscript in preparation.

2. S.P. Templeton, A.D. Buskirk, B. Law, B.J. Green, and D.H. Beezhold. "Role of germination in murine airway CD8-T cell responses to Aspergillus conidia". PLoS One. 2011. 6(4): e18777.

3. A.D. Buskirk, J.M. Hettick, I. Chipinda, B.F. Law, P.D. Siegel, J.E. Slaven, B.J. Green, and D.H. Beezhold. "Fungal pigments inhibit the matrix-assisted laser desorption/ionization time-of-flight mass spectrometry analysis of darkly pigmented fungi”. Anal. Biochem. 2011. 411(1):122-8.

4. J.M. Hettick, B.J. Green, A.D. Buskirk, J.E. Slaven, M.L. Kashon, and D.H. Beezhold. "Discrimination of fungi by MALDI-TOF mass spectrometry". Rapid Characterization of Microorganisms by Mass Spectrometry, 2011. 30-55.

5. S.P. Templeton, A.D. Buskirk, B.J. Green, D.H. Beezhold, D. Schmechel. "Murine Models of Airway Fungal Exposure and Allergic Sensitization". Medical Mycology. 2009. $48(2) 217-228$.

6. J.M. Hettick, B.J. Green, A.D. Buskirk, M.L. Kashon, J.E. Slaven, E. Janotka, F.M. Blachere, D. Schmechel, and D.H. Beezhold. "Discrimination of Penicillium isolates by 
matrix-assisted laser desorption/ionization time-of-flight mass spectrometry fingerprinting." Rapid Commun. Mass Spectrom. 2008. (16) 2555-2560.

7. J.M. Hettick, B.J. Green, A.D. Buskirk, M.L. Kashon, J.E. Slaven, E. Janotka, F.M. Blachere, D. Schmechel, and D.H. Beezhold. 'Identification of Aspergillus isolates at the species and strain level by matrix-assisted laser desorption/ionization time-of-flight mass spectrometry fingerprinting." Anal. Biochem. 2008. (380) 276-281.

\section{TEACHING EXPERIENCE}

Spring 2011

Fall 2011, 09, 07

\section{WEST VIRGINIA UNIVERSITY}

Department of Microbiology, Immunology, and Cell Biology Morgantown, WV

Teaching Assistant

Medical Technology Microbiology/Immunology Laboratory

- Lectured students in the Medical Technology professional program on background, concepts, and methods for various clinical microbiology and immunology exercises.

WEST VIRGINIA UNIVERSITY

Department of Microbiology, Immunology, and Cell Biology Morgantown, WV

Teaching Assistant

Dental Microbiology/Immunology Laboratory

- Lectured students in the School of Dentistry on background, concepts, and methods for various microbiology and immunology exercises. 